University of Tennessee Health Science Center

UTHSC Digital Commons

\title{
An Investigation into Clinically Relevant Determinants of Azole Resistance in Candida albicans
}

Andrew T. Nishimoto

University of Tennessee Health Science Center

Follow this and additional works at: https://dc.uthsc.edu/dissertations

Part of the Medicine and Health Sciences Commons, and the Molecular Biology Commons

\section{Recommended Citation}

Nishimoto, Andrew T. (0000-0003-1244-4656), "An Investigation into Clinically Relevant Determinants of Azole Resistance in Candida albicans" (2019). Theses and Dissertations (ETD). Paper 493.

http://dx.doi.org/10.21007/etd.cghs.2019.0486.

This Dissertation is brought to you for free and open access by the College of Graduate Health Sciences at UTHSC Digital Commons. It has been accepted for inclusion in Theses and Dissertations (ETD) by an authorized administrator of UTHSC Digital Commons. For more information, please contact jwelch30@uthsc.edu. 


\title{
An Investigation into Clinically Relevant Determinants of Azole Resistance in Candida albicans
}

\author{
Abstract \\ "Candida albicans is a commensal organism commonly colonizing the human gut and skin. As an \\ opportunistic pathogen, it can cause persistent and serious infections in individuals with compromised \\ immune systems, including the very young and elderly. Moreover, C. albicans can cause a wide spectrum \\ of diseases ranging from superficial mucosal infections to life-threatening invasions of the organs and \\ bloodstream. Candida species are the most common cause of invasive fungal disease, which is \\ associated with high mortality and imposes a heavy toll on the healthcare system. Over the last 30 years, \\ the azole antifungals have been a mainstay of antifungal therapy, being effective in a wide variety of \\ fungal infections and serving as the primary oral treatment option. However, increased use, inappropriate \\ dosing, and prolonged treatments have given rise to azole-resistant Candida albicans and other Candida \\ species. Resistance in C. albicans results from a combination of different mechanisms. Increased \\ expression of the efflux pump encoding genes CDR1, CDR2, and MDR1 as well as increased expression of \\ ERG11, encoding the azole target ( $14 \alpha$-lanosterol demethylase, also known as CYP51) are all primary \\ mechanisms of azole resistance that arise in azole-resistant clinical isolates. These changes are known \\ to be mediated through gain-of-function mutations in the genes of a fungal-specific transcription factor \\ family known as the zinc cluster transcription factors. Furthermore, genetic changes in the ergosterol \\ biosynthesis genes, ERG11 and ERG3, encoding a C-5 sterol desaturase, also contribute to clinical azole \\ resistance in $\mathrm{C}$. albicans. The interplay of these mechanisms can result in azole-resistance, treatment \\ failure, and ultimately, poorer outcomes in patients. Therefore, to improve healthcare outcomes, \\ understanding resistance development and the mechanisms that drive them in C. albicans is crucial. \\ Within a collection of predominantly fluconazole-resistant clinical isolates of $\mathrm{C}$. albicans, our lab had \\ previously characterized most known mechanisms of azole resistance present in each isolate. Increased \\ CDR1 expression in isolates lacking TAC1 gain-of-function mutations coupled with recent literature \\ suggesting a role of the Mrr2 zinc cluster transcription factor in azole resistance lead us to sequence and \\ test mutations in the MRR2 gene in across this collection. By placing mutant MRR2 alleles in azole- \\ susceptible backgrounds, we hoped to measure the contribution of MRR2 mutations to azole resistance \\ through changes in CDR1 expression and fluconazole minimum inhibitory concentrations (MICs). Counter \\ to what has been recorded in the literature, we found no evidence that mutations in MRR2 impact either \\ CDR1 expression or azole susceptibility in C. albicans. This is a novel finding correcting a previous \\ mistaken paradigm of a clinically relevant mechanism driving resistance in $\mathrm{C}$. albicans. Next we more \\ closely examined the role of ERG11 mutations found in clinical isolates. Though the contributory effects \\ of ERG11 mutations to azole resistance had been quantified, the specific biochemical impact of these \\ mutations on enzyme function and ligand-binding interaction have only recently come to light. Here we \\ introduced additional CaCYP51 amino acid substitution mutants (D278N and Y132H) in C. albicans" "vi" \\ "and tested our entire collection of CaCYP51 mutant strains to determine their in vitro azole \\ susceptibilities in the context of these findings. In general, we observed differences in the fluconazole and \\ voriconazole MICs between CaCYP51 amino acid substitutions. In contrast, MICs to itraconazole showed \\ a small, fairly consistent increase in MIC across tested CaCYP51 strains and MICs to posaconazole did \\ not increase at all over the wild type except for the G448E substitution, suggesting posaconazole \\ possesses the best in vitro activity against these CaCYP51 mutants. Overall, we also revealed that not all \\ ERG11 mutations confer azole resistance through decreased binding interactions with the target and the \\ azole drug, suggesting that CaCYP51 amino acid substitutions may instead interact with other associated \\ proteins to confer resistance. Furthermore, it was discovered that many ERG11 mutations from clinical \\ isolates result in low catalytic turnover of the enzyme, which is crucial to normal rates of ergosterol \\ production in a healthy cell. Though preliminary results of growth in CaCYP51 mutant strains does not \\ support attenuated fitness in competitive assay, the findings here prove that some clinical ERG11
}


mutations result in diminished enzyme function." We also sequenced the collection of clinical isolates and discovered an A351V Erg3 amino acid substitution in our azole-resistant isolates and predominantly in those with multiple ERG11 mutations. This suggested a possible connection between CaCYP51 mutants and amino acid substitutions in Erg3. As the proteins encoded for by the ERG11 and ERG3 genes are involved in the same ergosterol biosynthesis pathway, defects in Erg11 enzyme function might be expected to impact accumulation of substrates of Erg3, specifically, precursors of the toxic sterol metabolite $14 \alpha$-methylergosta- 8,24(28)-dien-3 $\beta, 6 \alpha$-diol. By testing ERG11 mutant strains with and without the ERG3A351V allele in growth competition experiments, we hoped to observe a conferred fitness benefit by the ERG3 mutation. Interestingly, we were unable to generate one of our selected ERG11 mutants with the poorest catalytic turnover in the absence of the A351V amino acid change in Erg3. Future investigation by other lab members is needed to determine if ERG3 mutations can indirectly influence azole susceptibility through permissive mutation." "Lastly, we tested susceptibility of our clinical collection to the new tetrazole antifungals VT-1161 and VT-1598, which have been reported to exhibit potent activity against azole-resistant $\mathrm{C}$. albicans and a host of other fungal species. We additionally investigated determinants of resistance to the two new agents by obtaining susceptibilities to $\mathrm{C}$. albicans strains containing individual known mechanisms of azole resistance. While susceptibility to VT-1161 was reduced when CDR1 and MDR1 were overexpressed, VT-1598 seemed unaffected by any tested resistance mechanism. Importantly, both retained activity against a significant portion of mutant ERG11 strains. VT-1598 MICs were not affected by any single mechanism of resistance. However, screening of our azole-resistant clinical isolates identified five isolates with greatly elevated MICs to all tested agents. While one of these isolates possesses an ERG3 nonsense mutation that likely explains its pan-azole resistant profile, the other four isolates do not uniquely overexpress known resistance genes or possess known gene mutations that might explain their resistance. This finding suggests that there are determinants of azole resistance that are as yet undiscovered in C. albicans."

\section{Document Type}

Dissertation

Degree Name

Doctor of Philosophy (PhD)

\section{Program}

Pharmaceutical Sciences

\section{Research Advisor}

P. David Rogers, Pharm.D., Ph.D.

\section{Keywords}

Azole, Candida albicans, ERG11, ERG3, MRR2, Resistance

\section{Subject Categories}

Medicine and Health Sciences | Molecular Biology 


\title{
An Investigation into Clinically Relevant Determinants of Azole Resistance in Candida albicans
}

\author{
A Dissertation \\ Presented for \\ The Graduate Studies Council \\ The University of Tennessee \\ Health Science Center
}

In Partial Fulfillment

Of the Requirements for the Degree

Doctor of Philosophy

From The University of Tennessee

By

Andrew T. Nishimoto

May 2019 
Chapter 2 and Chapter 5 (C) 2019 by American Society for Microbiology All other material (C) 2019 by Andrew T. Nishimoto All rights reserved. 


\section{DEDICATION}

To Mom, Dad, and Sushma. 


\section{ACKNOWLEDGEMENTS}

I do acknowledge that there are simply too many people to acknowledge. To my mentor, collaborators, colleagues, labmates, and friends. To the fungal pathogens group, the department, the administration, the college and the university. Ten years is far too short a time to spend among such excellent people.

"I don't know half of you half as well as I should I like, and I like less than half of you half as well as you deserve." - Bilbo Baggins

J.R.R. Tolkien, The Fellowship of the Ring, 1954

Finally, I would like to thank Dr. Daniel Diekema from the University of Iowa for generously sending us the clinical isolates of C. albicans used in Chapters 2, 3, 4, and 5. All work was supported by NIH grant R01 AI058145 to P.D.R.

Thank you, everyone. 


\begin{abstract}
Candida albicans is a commensal organism commonly colonizing the human gut and skin. As an opportunistic pathogen, it can cause persistent and serious infections in individuals with compromised immune systems, including the very young and elderly. Moreover, C. albicans can cause a wide spectrum of diseases ranging from superficial mucosal infections to life-threatening invasions of the organs and bloodstream. Candida species are the most common cause of invasive fungal disease, which is associated with high mortality and imposes a heavy toll on the healthcare system. Over the last 30 years, the azole antifungals have been a mainstay of antifungal therapy, being effective in a wide variety of fungal infections and serving as the primary oral treatment option. However, increased use, inappropriate dosing, and prolonged treatments have given rise to azole-resistant Candida albicans and other Candida species.
\end{abstract}

Resistance in C. albicans results from a combination of different mechanisms. Increased expression of the efflux pump encoding genes $C D R 1, C D R 2$, and $M D R 1$ as well as increased expression of ERG11, encoding the azole target (14 $\alpha$-lanosterol demethylase, also known as CYP51) are all primary mechanisms of azole resistance that arise in azole-resistant clinical isolates. These changes are known to be mediated through gain-of-function mutations in the genes of a fungal-specific transcription factor family known as the zinc cluster transcription factors. Furthermore, genetic changes in the ergosterol biosynthesis genes, $E R G 11$ and $E R G 3$, encoding a C-5 sterol desaturase, also contribute to clinical azole resistance in C. albicans. The interplay of these mechanisms can result in azole-resistance, treatment failure, and ultimately, poorer outcomes in patients. Therefore, to improve healthcare outcomes, understanding resistance development and the mechanisms that drive them in C. albicans is crucial.

Within a collection of predominantly fluconazole-resistant clinical isolates of $C$. albicans, our lab had previously characterized most known mechanisms of azole resistance present in each isolate. Increased CDR1 expression in isolates lacking TAC1 gain-of-function mutations coupled with recent literature suggesting a role of the Mrr2 zinc cluster transcription factor in azole resistance lead us to sequence and test mutations in the $M R R 2$ gene in across this collection. By placing mutant $M R R 2$ alleles in azolesusceptible backgrounds, we hoped to measure the contribution of $M R R 2$ mutations to azole resistance through changes in $C D R 1$ expression and fluconazole minimum inhibitory concentrations (MICs). Counter to what has been recorded in the literature, we found no evidence that mutations in MRR2 impact either CDR1 expression or azole susceptibility in C. albicans. This is a novel finding correcting a previous mistaken paradigm of a clinically relevant mechanism driving resistance in C. albicans.

Next we more closely examined the role of ERG11 mutations found in clinical isolates. Though the contributory effects of ERG11 mutations to azole resistance had been quantified, the specific biochemical impact of these mutations on enzyme function and ligand-binding interaction have only recently come to light. Here we introduced additional CaCYP51 amino acid substitution mutants (D278N and Y132H) in C. albicans 
and tested our entire collection of CaCYP51 mutant strains to determine their in vitro azole susceptibilities in the context of these findings. In general, we observed differences in the fluconazole and voriconazole MICs between CaCYP51 amino acid substitutions. In contrast, MICs to itraconazole showed a small, fairly consistent increase in MIC across tested CaCYP51 strains and MICs to posaconazole did not increase at all over the wild type except for the G448E substitution, suggesting posaconazole possesses the best in vitro activity against these CaCYP51 mutants. Overall, we also revealed that not all ERG11 mutations confer azole resistance through decreased binding interactions with the target and the azole drug, suggesting that CaCYP51 amino acid substitutions may instead interact with other associated proteins to confer resistance. Furthermore, it was discovered that many ERG11 mutations from clinical isolates result in low catalytic turnover of the enzyme, which is crucial to normal rates of ergosterol production in a healthy cell. Though preliminary results of growth in CaCYP51 mutant strains does not support attenuated fitness in competitive assay, the findings here prove that some clinical ERG11 mutations result in diminished enzyme function.

We also sequenced the collection of clinical isolates and discovered an A351V Erg3 amino acid substitution in our azole-resistant isolates and predominantly in those with multiple ERG11 mutations. This suggested a possible connection between CaCYP51 mutants and amino acid substitutions in Erg3. As the proteins encoded for by the ERG11 and ERG3 genes are involved in the same ergosterol biosynthesis pathway, defects in Erg11 enzyme function might be expected to impact accumulation of substrates of Erg3, specifically, precursors of the toxic sterol metabolite $14 \alpha$-methylergosta8,24(28)-dien-3 $\beta, 6 \alpha$-diol. By testing ERG11 mutant strains with and without the $E R G 3^{\mathrm{A} 351 \mathrm{~V}}$ allele in growth competition experiments, we hoped to observe a conferred fitness benefit by the $E R G 3$ mutation. Interestingly, we were unable to generate one of our selected ERG11 mutants with the poorest catalytic turnover in the absence of the A351V amino acid change in Erg3. Future investigation by other lab members is needed to determine if ERG3 mutations can indirectly influence azole susceptibility through permissive mutation.

Lastly, we tested susceptibility of our clinical collection to the new tetrazole antifungals VT-1161 and VT-1598, which have been reported to exhibit potent activity against azole-resistant $C$. albicans and a host of other fungal species. We additionally investigated determinants of resistance to the two new agents by obtaining susceptibilities to $C$. albicans strains containing individual known mechanisms of azole resistance. While susceptibility to VT-1161 was reduced when CDR1 and MDR 1 were overexpressed, VT-1598 seemed unaffected by any tested resistance mechanism. Importantly, both retained activity against a significant portion of mutant ERG11 strains. VT-1598 MICs were not affected by any single mechanism of resistance. However, screening of our azole-resistant clinical isolates identified five isolates with greatly elevated MICs to all tested agents. While one of these isolates possesses an ERG3 nonsense mutation that likely explains its pan-azole resistant profile, the other four isolates do not uniquely overexpress known resistance genes or possess known gene mutations that might explain their resistance. This finding suggests that there are determinants of azole resistance that are as yet undiscovered in C. albicans. 


\section{TABLE OF CONTENTS}

CHAPTER 1. INTRODUCTION ...................................................................................

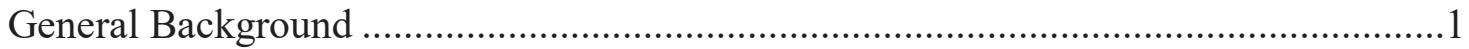

Biology of Candida albicans ....................................................................................2

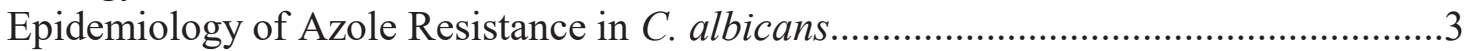

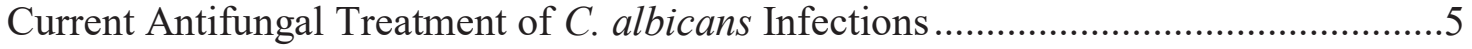

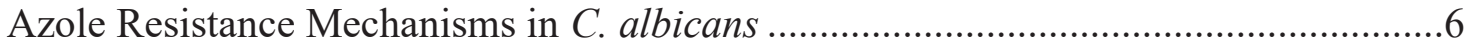

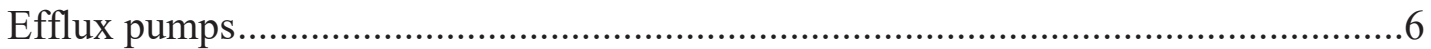

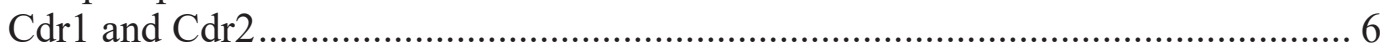

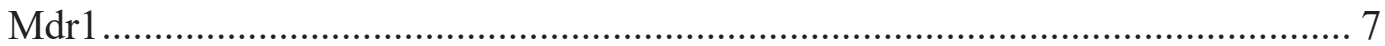

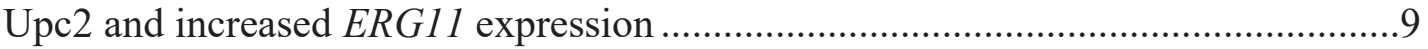

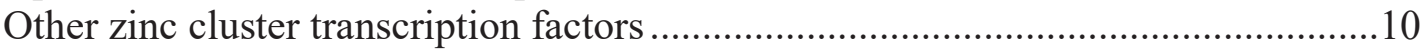

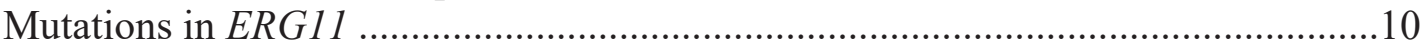

Mutations in ERG3 and alternative sterol biosynthesis ...................................... 11

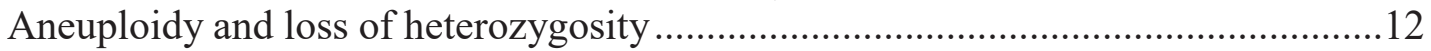

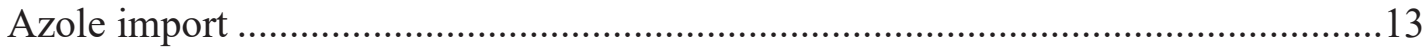

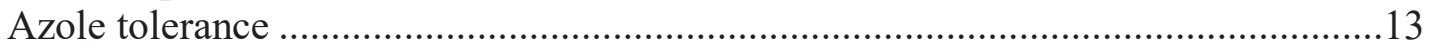

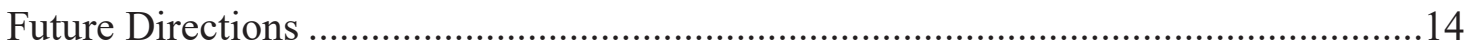

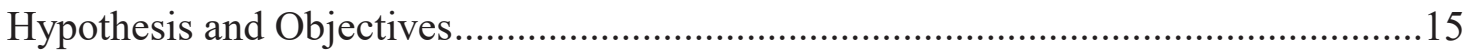

\section{CHAPTER 2. THE CONTRIBUTION OF CLINICALLY-DERIVED}

MUTATIONS IN THE GENE ENCODING THE ZINC CLUSTER

TRANSCRIPTION FACTOR MRR2 TO FLUCONAZOLE ANTIFUNGAL RESISTANCE AND CDR1 EXPRESSION IN CANDIDA ALBICANS ....................19

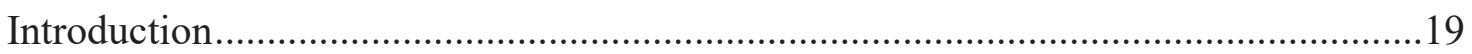

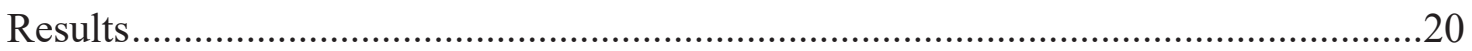

SNPs in MRR2 among fluconazole-resistant clinical isolates of $C$. albicans ............20

Fluconazole susceptibility in $M R R 2$ mutant strains ...............................................21

$M R R 2$ mutant strains do not overexpress $C D R 1$ when expressed from the $M R R 2$

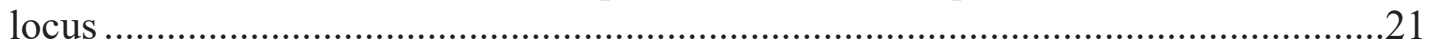

Amino acid substitutions S466L, A468G, S469T, and T470N do not influence

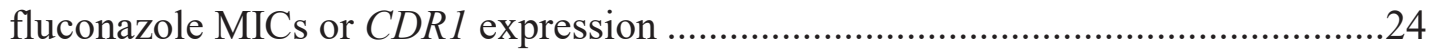

Activated Mrr2 does not appear to regulate $M R R 2$ expression ...............................27

Overexpression of wildtype or mutant $M R R 2$ in $C$. albicans strain does not affect

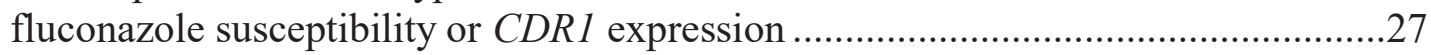

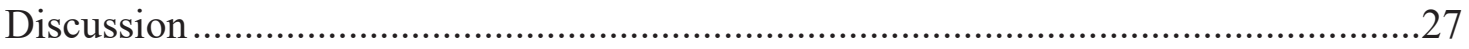

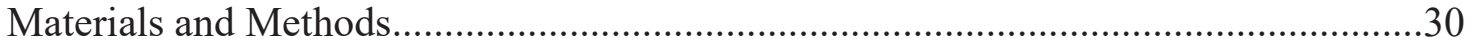

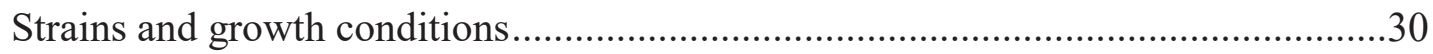

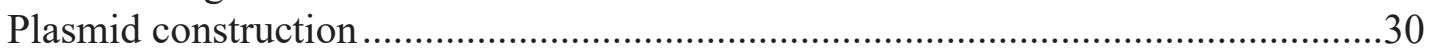

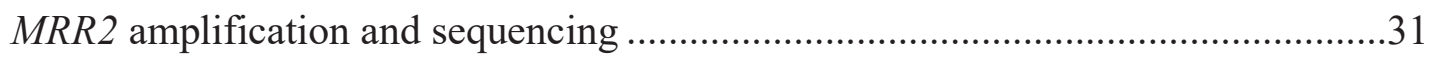

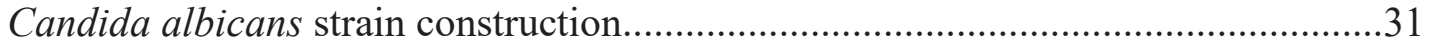

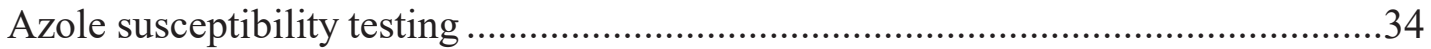

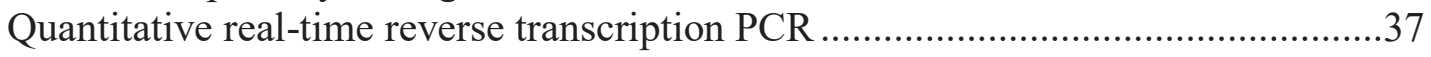

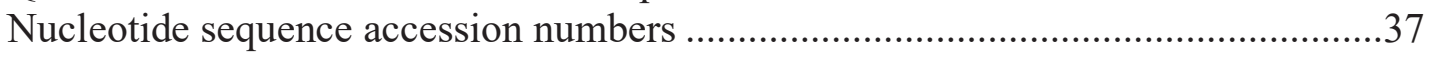




\section{CHAPTER 3. MUTATIONS IN ERG11 HAVE VARYING CONTRIBUTIONS TO AZOLE RESISTANCE, ERG11 PROTEIN FUNCTION, AND COMPETITIVE GROWTH FITNESS OF THE CELL ............................................38}

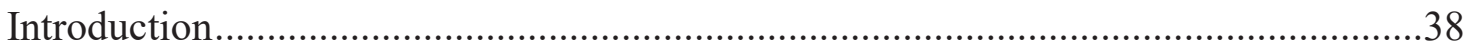

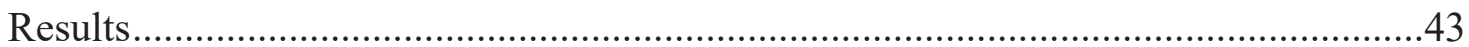

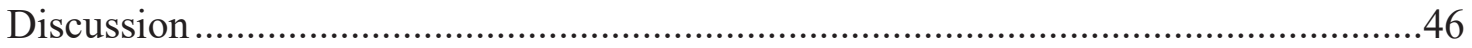

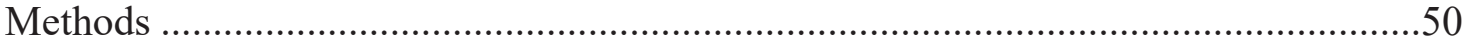

Construction of $C$. albicans CYP51 mutant strains ............................................50

Susceptibility testing of $C$. albicans CYP51 mutant strains ...................................52

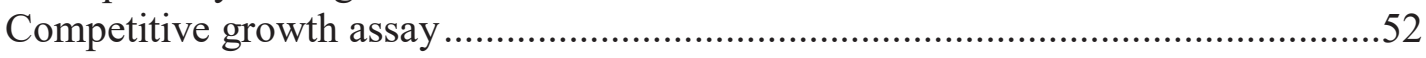

\section{CHAPTER 4. THE ROLE OF ERG3 MUTATIONS TO AZOLE RESISTANCE} AND ERG11 MUTABILITY IN $C$. ALBICANS .......................................................53

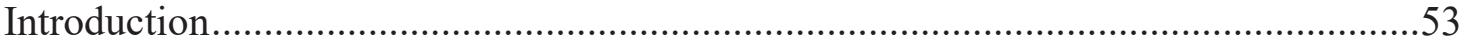

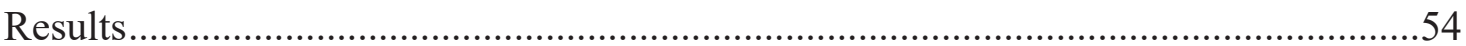

Frequency of Erg11 and Erg3 amino acid substitutions in C. albicans clinical

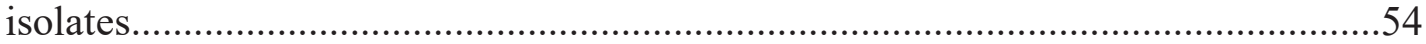

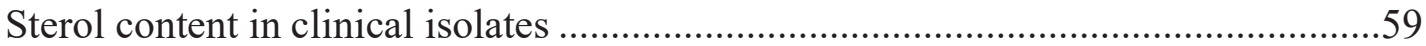

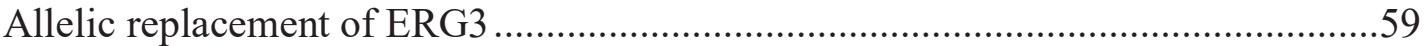

The A351V amino acid substitution in Erg3 does not directly affect in vitro fluconazole susceptibility............................................................................... 59 A homozygous ERG11 $1^{\mathrm{Y} 132 \mathrm{H}, \mathrm{K} 143 \mathrm{~T}}$ mutant is conditionally constructed in

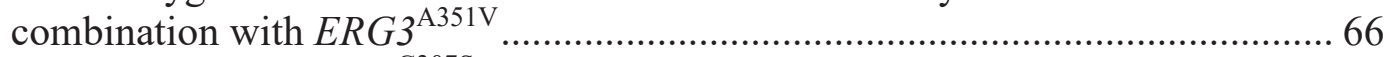

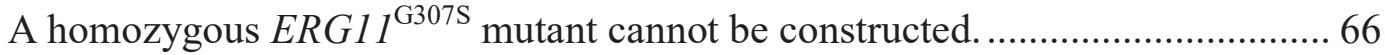

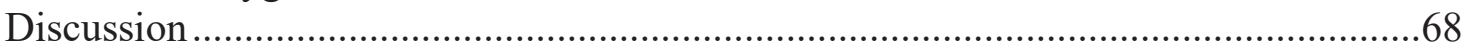

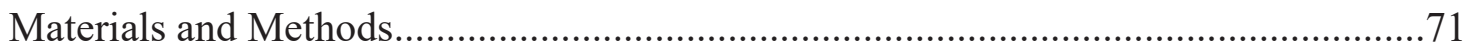

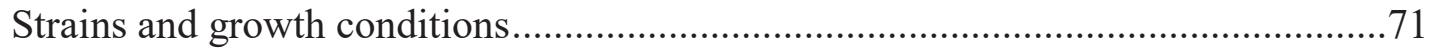

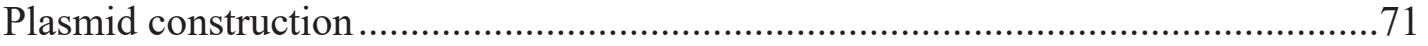

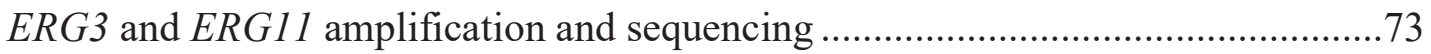

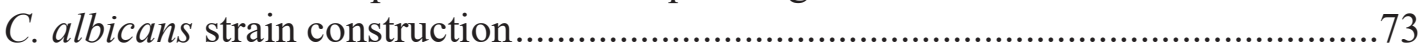

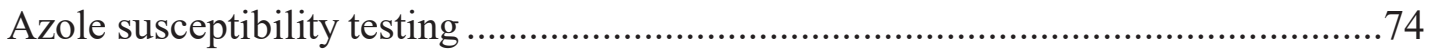

\section{CHAPTER 5. IN VITRO ACTIVITIES OF THE NOVEL INVESTIGATIONAL TETRAZOLE VT-1161 AND VT-1598 COMPARED TO THE TRIAZOLE ANTIFUNGALS AGAINST AZOLE-RESISTANCE STRAINS AND CLINICAL ISOLATES OF CANDIDA ALBICANS .............................................75}

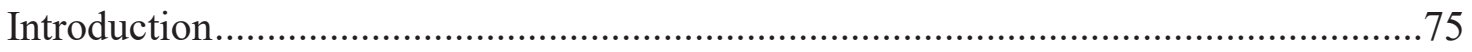

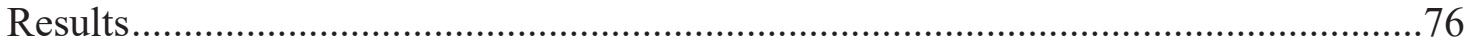

In vitro activity of VT-1161 and VT-1598 against fluconazole-susceptible and

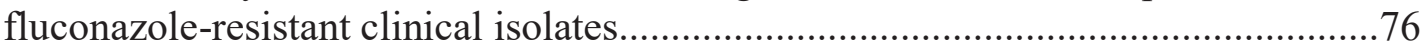
In vitro activity of VT-1161 and VT-1598 against strains with known azole

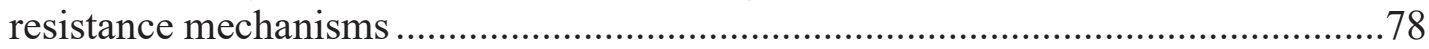

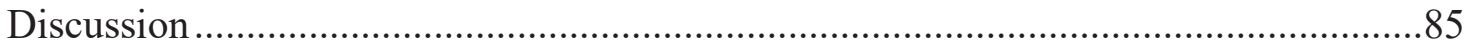

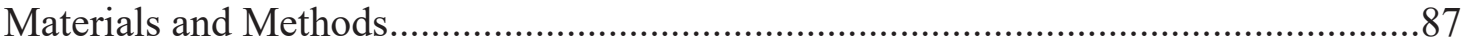

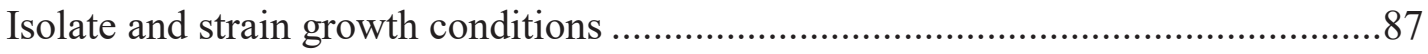




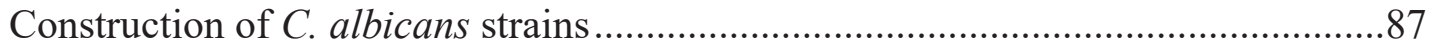

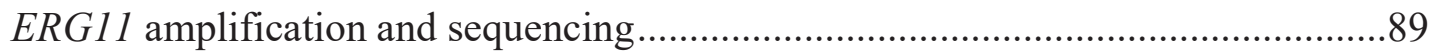

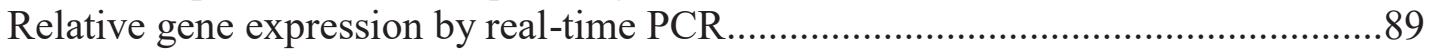

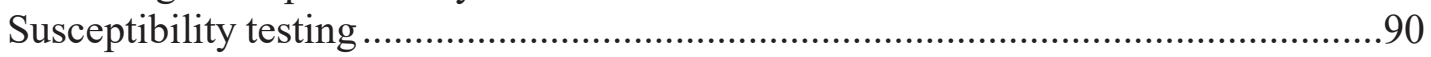

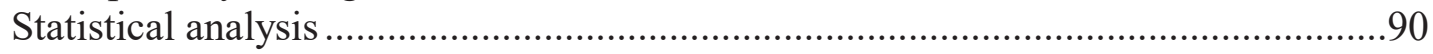

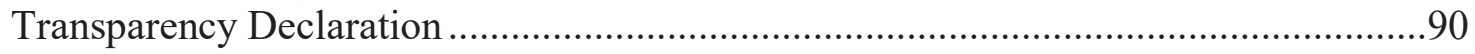

CHAPTER 6. CONCLUSIONS ............................................................................91

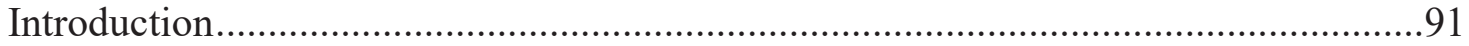

Summary and Conclusions of Chapter 2 ............................................................ 91

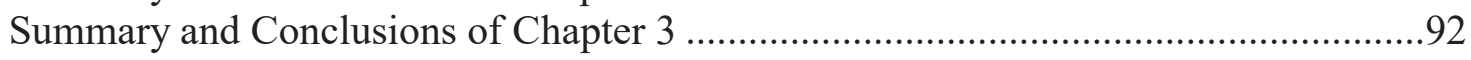

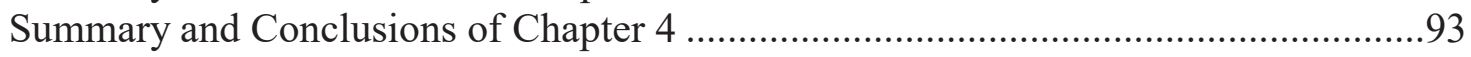

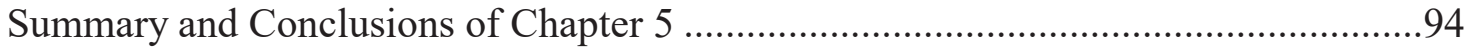

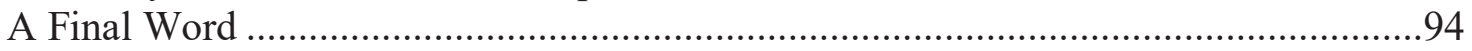

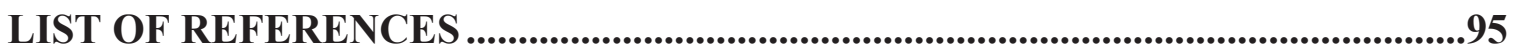

APPENDIX A. AMINO ACID SUBSTITUTIONS IN MRR2 CLINICAL C.

ALBICANS ISOLATES ...................................................................................118

APPENDIX B. SUPPLEMENTARY DATA TABLES TO CHAPTER 5................120

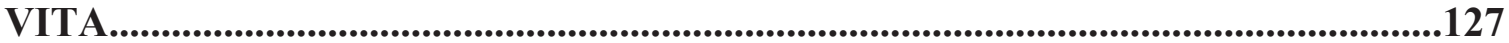




\section{LIST OF TABLES}

Table 2-1. Mrr2 amino acid substitutions found in azole-resistant clinical isolates

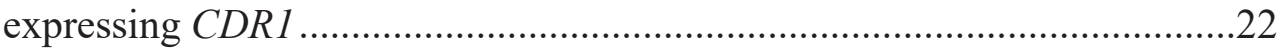

Table 2-2. Fluconazole susceptibilities for MRR2 mutant strains ..................................23

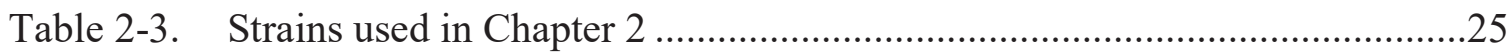

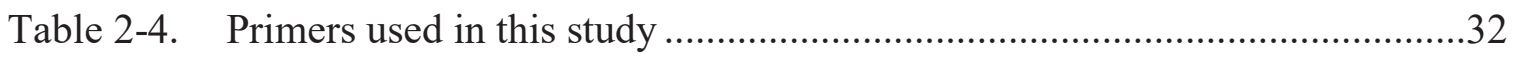

Table 3-1. Yield and relative velocities of CaCYP51 proteins .......................................41

Table 3-2. Residual CYP51 activity in the presence of $4 \mu \mathrm{M}$ triazole antifungal .........42

Table 3-3. Triazole antifungal $\mathrm{IC}_{50}$ values obtained with $\mathrm{CaCYP} 51$ proteins ................44

Table 3-4. CLSI MICs $\left(\mu \mathrm{g} \mathrm{mL}^{-1}\right)$ for fluconazole, voriconazole, itraconazole, and posaconazole against strains containing CaCYP51 amino acid

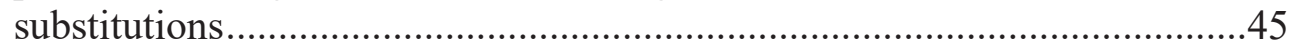

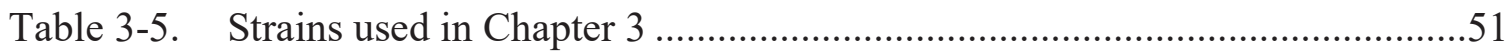

Table 4-1. Fluconazole MIC, ergosterol content, and mutations in ERG11 and $E R G 3$ in clinical isolates of $C$. albicans....................................................57

Table 4-2. Percent total cell membrane sterol content composition in C. albicans

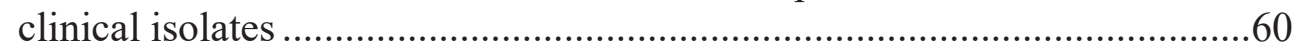

Table 4-3. 24-hour fluconazole MICs for C. albicans strains in Chapter 4 ...................67

Table 4-4. Primers used in Chapter 4 ………………...............................................72

Table 5-1. Geometric mean MICs, $\mathrm{MIC}_{50}$, $\mathrm{MIC}_{90}$, and ranges $(\mu \mathrm{g} / \mathrm{mL})$ for tested compounds against 68 clinical isolates of $C$. albicans ................................77

Table 5-2. Constructed strains used in Chapter 5......................................................8

Table A-1. Amino acid substitutions in Mrr2 clinical C. albicans isolates ...................118

Table B-1. Minimum inhibitory concentration values for VT-1161, VT-1598, fluconazole, voriconazole, itraconazole, posaconazole, and known azole resistance mechanisms in clinical C. albicans isolates ..............................120

Table B-2. MICs for VT-1161, VT-1598, fluconazole, voriconazole, itraconazole, and posaconazole against $E R G 11$ mutant strains .....................................125

Table B-3. Primers used in Chapter 5 ......................................................................126 


\section{LIST OF FIGURES}

Figure 1-1. Expression levels of $C D R 1, C D R 2, M D R 1$, and $E R G 11$ in 63

fluconazole-resistant clinical isolates

Figure 2-1. Fold-change in expression levels of $M R R 2$ mutant strains compared to SC5314

Figure 2-2. Fold-change in $C D R 1$ and $M R R 2$ expression compared to SC5314. .28

Figure 2-3. Representative schematic of $M R R 2$ mutant strain construction in $C$. albicans.....

Figure 3-1. Reported locations of CaCYP51 amino acid substitutions in C. albicans ....40

Figure 3-2. Competive growth fitness testing of Erg11 mutants

Figure 4-1. Normal ergosterol biosynthesis pathway inhibition by the azole antifungals in C. albicans.

Figure 4-2. Ergosterol biosynthesis pathway inhibition by the azole antifungals in $C$. albicans with defective $\mathrm{C}-5$ sterol desaturase ...... . .56

Figure 4-3. Normal ergosterol biosynthesis pathway in C. albicans.

Figure 4-4. Alternative sterol biosynthesis pathway in C. albicans cells with defective C-5 sterol desaturase......

Figure 4-5. Proposed sterol biosynthesis pathway in C. albicans cells with ERG11 mutations resulting in slow Erg11 enzyme function

Figure 5-1. Comparison of the MICs of VT-1161 and VT-1598 against the MICs of triazoles

Figure 5-2. MICs of tested azole compounds against strains with individual known azole resistance mechanisms

Figure 5-3. Relative fold-change compared to SC5314 in MIC of various azole antifungal agents against strains containing single and double ERG11 mutations . 


\section{LIST OF ABBREVIATIONS}

$\begin{array}{ll}\text { Ca } & \text { Candida albicans } \\ \text { CLSI } & \text { Clinical Laboratory and Standards Institute } \\ \text { CRISPR } & \text { Clustered Regularly Interspaced Short Palindromic Repeats } \\ \text { CYP } & \text { cytochrome P450 } \\ \text { EUCAST } & \text { European Committee on Antimicrobial Susceptibility Testing } \\ \text { GM } & \text { geometric mean } \\ \text { GOF } & \text { gain-of-function } \\ \text { LOF } & \text { loss-of-function } \\ \text { LOH } & \text { loss-of-heterozygosity } \\ \text { MIC } & \text { minimum inhibitory concentration } \\ \text { PCR } & \text { polymerase chain reaction } \\ \text { qPCR } & \text { real time quantitative polymerase chain reaction } \\ \text { spp. } & \text { species } \\ \text { ZCF } & \text { zinc cluster transcription factor }\end{array}$




\section{CHAPTER 1. INTRODUCTION}

\section{General Background}

Manifestations of Candida infections range from superficial mucosal and dermal infections such as oral thrush and vaginal yeast infections to disseminated bloodstream infections and are associated with high mortality rates (upwards of $>40 \%)(1-5)$. Candida infections require extreme attention in immunocompromised individuals, such as AIDS patients, transplant recipients, patients undergoing chemotherapy or immunosuppression therapies, in which they cause life-threatening invasive infections (68). Studies have suggested that healthy individuals with implanted medical devices, those who experienced major trauma, and patients requiring extended stays in intensive care units are at equal risk of acquiring Candida infection (9). Global estimates suggest that invasive candidiasis occur in $\sim 700,000$ cases which clearly exceeds $\sim 250,000$ cases of invasive aspergillosis (10).

In the USA, Candida spp. remain the fourth leading cause of nosocomial bloodstream infections and the number three cause of bloodstream infections in the intensive care unit (ICU) (11). Candida spp. are also the third leading cause of centralline-associated bloodstream infections and the second leading cause of catheterassociated urinary tract infections in the U.S. $(12,13)$. Of all the Candida species, $C$. albicans dominates almost all patient groups and disease manifestations in terms of incidence followed by C. glabrata, C. parapsilosis, C. tropicalis, and C. krusei (14). It is pertinent to mention here that there has been considerable change in species distribution, dependent upon geographical location and patient population, with a decrease in the proportion of C. albicans and an increase in C. glabrata, C. parapsilosis, and other nonalbicans Candida species (14). C. glabrata is the second most common species in the United States and northwestern Europe in the non-outbreak setting $(15,16)$. However, $C$. parapsilosis and/or C. tropicalis are much more frequently encountered than C. glabrata in Latin America, Southern Europe, India and Pakistan (17-23). Of the five major Candida spp., C. krusei is the least common, and it is most often found among patients with underlying haematological malignancies with prior antifungal exposure $(24,25)$. Another worrisome multidrug resistant species that is emerging globally is C. auris (26).

C. albicans is a member of native human microflora and as a commensal organism colonizes the gastrointestinal (GI) tract, reproductive tract, oral cavity, and skin of most humans (27). Failure to identify a possible environmental reservoir for $C$. albicans suggests that this species is exquisitely adapted to healthy mammalian hosts. However, this benign commensal colonization can become pathogenic due to shifts in $\mathrm{pH}$ and oxygen levels, alterations in host microbiota (e.g. from antibiotic usage), or changes in the host immune response (e.g. during stress, infection by another microbe, or immunosuppressant therapy) (28). Rapid increase in number of Candida infections may be attributed to availability of modern medical treatments including excessive usage of broad-spectrum antibiotics, anticancer therapy, solid organ transplantation, presence of 
indwelling catheters, lack of awareness among multiple medical specialties, and poor performance of routine microbiological tests.

Fluconazole is the first line of antifungal drug available for an effective treatment for invasive candidiasis and is the recommended treatment for most infections (29). While antifungal resistance in $C$. albicans is uncommon relative to species like $C$. glabrata, gradual use of fluconazole for long term prophylaxis or treatment may lead to selective pressure resulting in emergence of secondary resistance in otherwise susceptible strains/species. Overall, fluconazole resistance (MIC $\geq 8 \mu \mathrm{g} / \mathrm{ml}$ ) in C. albicans ranges between $0 \%$ and $3 \%$ (30-34). Although overall azole resistance of naïve $C$. albicans isolates is rare, a steady increase in reports of azole resistance, resulting in therapeutic failures, has been a matter of serious clinical concern. In the current review, we aim to address the magnitude of azole-resistance in C. albicans and the underlying resistance mechanism implicated in the development of azole resistance and highlight the therapeutic concerns.

\section{Biology of Candida albicans}

Candida albicans possess key virulence traits that not only differentiate it from other Candida species but also help it in establishing itself as a successful pathogen. It secretes a number of proteases, phospholipases, esterases and lipases in the context of invasive infection that helps in promoting degradation of host connective tissue, cleavage of host immune defence proteins thereby leading to nutrition acquisition, invasion and evasion of pathogen from host innate immune response $(35,36)$.

C. albicans can adapt to use alternative carbon sources due to its ability to successfully thrive in heterogenous carbon microenvironment in the host. The versatility of metabolic elasticity of this species helps in morphogenic transition between unicellular yeast cells, pseudohyphae, and hyphae thereby promoting invasion (37-41). This is in contrast to C. glabrata, which has the intrinsic inability to form hyphae and does not require this morphogenic change in order to establish invasive disease. C. albicans has the ability for cell wall remodeling, white opaque switching and can form highly structured biofilms composed of multiple cell types (i.e. round yeast-form cells; oval pseudohyphal cells; and elongated, cylindrical hyphal cells) encased in an extracellular matrix (42-46). Due to their effective adherence and invasion in endothelial and epithelial cells they can easily disseminate into the bloodstream. C. albicans can invade host epithelial cells either through forced hyphal-mediated invasion or by induced endocytosis into endothelial and epithelial cells (47-50). It is pertinent to mention here that all Candida species exhibit different antifungal susceptibility patterns depending upon their site of isolation, geographical location, and prior antifungal exposure. C. albicans and $C$. parapsilosis are most frequently susceptible to all antifungals available. On the other hand, C. tropicalis might exhibit fluconazole resistance while maintaining susceptible profile to amphotericin B and echinocandins. Interestingly, C. glabrata tends to display high azole MICs while retaining amphotericin B and echinocandin susceptibility. $C$. 
lusitaniae, on the other hand, is resistant to Amphotericin B while the emerging fungal threat $C$. auris is multidrug resistant.

\section{Epidemiology of Azole Resistance in C. albicans}

In vitro susceptibility testing of yeasts and moulds helps in not only selecting the most clinically active antifungal agent but also in detecting resistance rates. The Clinical and Laboratory Standards Institute (CLSI) and European Committee on Antimicrobial Susceptibility Testing (EUCAST) methods are reference methods to detect in vitro resistance of yeasts and moulds against various antifungal agents. Resistance can be classified as microbiologic or clinical. Microbiologic resistance is defined by the situation when concentration of antifungal agent required to inhibit the growth of the pathogen is higher than the range seen for wild-type strains due to presence of an acquired or innate mutational resistance mechanism. Clinical resistance, on the other hand, is denominated by therapeutic failure in spite of administering high doses of the antifungal agent.

Emergence of azole resistance in Candida species, especially those originating from patients on prolonged azole therapy, have become a worldwide problem thereby stressing the need for antifungal stewardship. Decreased in vitro susceptibility of $C$. albicans to azoles such as miconazole, econazole, and clotrimazole has been recognized since the late 1970s $(51,52)$. Horsburgh and Kirkpatrick in 1983 reported two of 21 patients with chronic mucocutaneous candidiasis on ketoconazole treatment suffering clinical relapses associated with C. albicans possessing high ketoconazole MICs (53). Similarly, high ketoconazole MICs were subsequently reported by Warnock et al. and Smith et al. in similar cases $(54,55)$. These relatively ketoconazole-resistant isolates demonstrated decreased in vitro susceptibility to miconazole, itraconazole, fluconazole, and tioconazole. There have also been reports of mucosal candidiasis due to $C$. albicans with reduced in vitro and in vivo responsiveness to fluconazole, ketoconazole, and clotrimazole in HIV-infected patients $(56,57)$. Evans et al. reported three cases of treatment failure in invasive mycoses due to fluconazole resistant C. albicans (58).

Most of the data related to the epidemiology of antifungal susceptibility profile of Candida spp. originates mainly from studies carried out in the USA and Europe. Cleveland et al., in 2012 showed an azole resistance rate of about $2.3 \%$ in C. albicans bloodstream isolates (59). The SENTRY surveillance data from 2008-2009 investigated 1239 bloodstream Candida isolates from 79 medical centers throughout the world and reported variation in Candida spp. distribution and antifungal resistance rates (32). Candida albicans $(\mathrm{n}=620,50 \%)$ was the most common species isolated in all age groups followed by $C$. glabrata and $C$. parapsilosis $(\mathrm{n}=215,17.4 \%)$, and $C$. tropicalis $(\mathrm{n}=122$, $9.8 \%$ ). None of the C. albicans isolates were resistant to any of the antifungal tested in this study. Interestingly, a recent SENTRY surveillance data analyzing 1514 Candida spp. collected from Europe (41.0\%), the Asia Pacific (24.5\%), North America (23.5\%), and Latin America (11.0\%) during 2013 revealed the similar pattern of Candida species isolation (15). However, azole antifungal resistance of about $0.4 \%$ and $0.3 \%$ in $C$. 
albicans $(\mathrm{n}=712)$ was noted against fluconazole and voriconazole, respectively. Another large population-based surveillance program, the ARTEMIS DISK Global Antifungal Surveillance Study was carried out from 1997 to 2007, analyzing 256,882 isolates of Candida spp. collected and tested at 142 study centers throughout the world (30). Of all 31 different Candida species isolated, C. albicans (65.3\%) was the most common followed by $C$. glabrata (10.2\% to $11.7 \%), C$. tropicalis, $(5.4 \%$ to $8.0 \%)$, and $C$. parapsilosis (4.8\% to 5.6\%). Of 128,625 isolates of C. albicans tested, 1.4\% were resistant to fluconazole. Of 1782 fluconazole-resistant $C$. albicans strains, $63.6 \%$ were cross resistant to voriconazole (30). A surveillance study was conducted in Denmark to evaluate the species distribution and in vitro antifungal susceptibility profile against fluconazole, itraconazole, amphotericin B and caspofungin in 303 episodes of fungaemia (60). Here again, $C$. albicans was the predominant species isolated followed by $C$. glabrata, $C$. tropicalis and $C$. parapsilosis. Of all the $C$. albicans tested, $3 \%(n=6)$ were resistant to fluconazole. In another Danish study, a 6-year nationwide study of fungemia from 2004-2009 reported fluconazole (MIC of $>4 \mu \mathrm{g} / \mathrm{ml}$ ) resistance rate of about $0.6 \%$ (7/1183 isolates) (61).

A recent study from Spain assessed the incidence and prevalence of antifungal resistance in bloodstream Candida infections (62). Interestingly, in a collection of 766 Candida strains, C. albicans (45.4\%) was the predominant species followed by $C$. parapsilosis (24.9\%), C. glabrata (13.4\%), C. tropicalis (7.7\%), C. krusei (2\%), and other Candida species (6.5\%). Of all C. albicans strains tested for antifungal susceptibility, $1 \%$ was resistant to fluconazole. A multi-center study from Belgium during 2013-2014 demonstrated eleven different Candida species, with $C$. albicans being the most prevalent (50.4\%), followed by C. glabrata $(27.3 \%)$, C. parapsilosis sensu lato $(9.8 \%)$, and C. tropicalis (5.6\%) (63). About 3.9\% $(\mathrm{n}=7)$ of C. albicans $(\mathrm{n}=179)$ isolates were found be fluconazole resistant here. A twenty-two year Norwegian national surveillance of candidaemia found $98.8 \%$ C. albicans isolates to be susceptible to fluconazole (64). Only $0.25 \%$ of $C$. albicans isolates were fluconazole resistant during 2004-2012.

Chapman et al., from Australia reported two (0.9\%) isolates of C. albicans that were resistant to fluconazole, cross-resistant to voriconazole and had non-wild type MICs of itraconazole $(>16 \mathrm{mg} / \mathrm{L})$ and posaconazole $(>8 \mathrm{mg} / \mathrm{L})(65)$. A national South African surveillance for candidemia from 2009-2010 revealed 98\% C. albicans isolates to be azole susceptible. However, a single fluconazole-resistant $C$. albicans isolate exhibited cross resistance to voriconazole (66). In a 2015 study conducted in ICU centers across India, 5.2\% of C. albicans strains sourced from ICU-acquired candidemias were identified as fluconazole-resistant (67).

Overall, the global data suggests that $C$. albicans remains the predominant infective organism identified in Candida-based infections. While reported resistance rates vary from study to study, collected surveillance data suggests that fluconazoleresistance and azole resistance rates in general for $C$. albicans remains relatively low. However, superficial mucosal $C$. albicans infections pose a considerable threat in immunocompromised populations, and the high morbidity and mortality associated with 
serious invasive Candida infections continues to burden our healthcare systems (68-70). As such we must not overlook $C$. albicans as a serious antimicrobial resistance threat.

\section{Current Antifungal Treatment of $C$. albicans Infections}

Currently, antifungal therapy revolves around three main classes of antifungal drugs: the azole antifungals, echinocandins, and polyene antifungals such as amphotericin B. The antimetabolite pyrimidine analog flucytosine is also used in treatment of certain invasive Candida infections and cryptococcal meningitis, but is limited to combination therapies with amphotericin B (29). The azole antifungals are a current mainstay of therapy for treatment and prophylaxis of both superficial and invasive candidiasis. Through inhibition of the ERG11-encoded protein 14a-lanosterol demethylase, the production of ergosterol normally required for membrane integrity is halted. In $C$. albicans, the inability to demethylate lanosterol via Erg11 shunts sterol biosynthesis production to other alternate biosynthesis pathways, resulting in the accumulation of $14 \alpha-$ methylergosta-8,24(28)-dien-3beta,6alpha-diol (71). The accumulation of this compound is toxic to the cell and results in the fungistatic effect seen with the azole antifungals (72).

While there are dozens of azole antifungal drugs sold commercially worldwide, ranging from research use to topical antifungal use in humans to agriculture, only a select few triazoles are typically used for treating invasive candidiasis in the United States. While recent updates to clinical practice guidelines now recommend the echinocandin class of antifungals in many invasive Candida infections, fluconazole remains a viable first-line alternative in many cases, such as in non-neutropenic patients with Candida bloodsteam infection (29). Voriconazole is another triazole considered as alternative or step-down therapy in many instances where fluconazole is also used, and the triazoles itraconazole and posaconazole have niche uses in invasive candidiasis, such as alternatives for oropharyngeal or esophageal candidiasis in fluconazole-refractory disease. Additionally, as currently no orally available echinocandin exists, fluconazole and the other triazoles are important for outpatient and step-down therapies for prophylaxis and treatment. The newly approved triazole prodrug isavuconazonium sulfate, while effective against Candida, including azole-resistant Candida species, does not currently have a place in treatment of invasive candidiasis and is instead reserved for the serious mould infections like aspergillosis and mucormycosis $(73,74)$.

Notably, a new subclass of azole antifungal compounds are in the drug pipeline. The tetrazole compounds, VT-1129, VT-1161 and VT-1598, so named because of an additional nitrogen on the five-membered azole ring that defines the class, are currently awaiting or undergoing clinical trials to treat a wide variety of fungal infections $(75,76)$. VT-1161, more specifically, is currently undergoing clinical trials as a treatment for recurrent vaginal candidiasis and may be a plausible future therapy against azole-resistant C. albicans.

There are many barriers to effective treatment with the azole antifungals in

invasive candidiasis. Improper dosing and inadequate drug delivery to the source of 
infection are obvious clinical faux pas to avoid, however, C. albicans and other Candida species have a variety of methods to survive even proper treatment practices. The next section details the specifics of the known azole resistance mechanisms in Candida albicans.

\section{Azole Resistance Mechanisms in C. albicans}

\section{Efflux pumps}

\section{Cdr1 and Cdr2}

Like many infecting organisms, $C$. albicans utilizes an efflux system to transport drugs extracellularly, thereby reducing intracellular drug concentrations. As such the overexpression of azole-targeting efflux pumps is a key mechanism driving azole resistance in $C$. albicans. The ATP-binding cassette (ABC) transporters Cdr1 and Cdr2 have both been well-documented as drivers of azole resistance and were first implicated in clinical azole resistance in isolates in HIV-infected patients with oropharyngeal candidiasis (77-79). In two separate studies, it was found that isolates of C. albicans taken from HIV-infected individuals had increased mRNA levels of $C D R 1$, which has been shown to confer resistance to the azole antifungals (79-81).

Importantly, the zinc-cluster transcription factor Tac1 was discovered to regulate the expression of both $C D R 1$ and $C D R 2$ and that a codominant mutation in the putative C-terminal activation domain conferred hyperactivity of the Tac1 transcription factor, leading to constitutive increased expression of $C D R 1$ and $C D R 2(82,83)$. In a comparison of azole-susceptible versus azole-resistant matched isolates, constitutive high expression of these transporters was suggested to be a result of both increased transcription and increased mRNA stability, the latter of which is tied to mRNA hyperadenylation and loss-of-heterozygosity at the poly(A) polymerase 1 locus $(84,85)$. Since then, multiple gain-of-function mutations in Tac1 leading to increased expression of the Cdr1 and Cdr2 efflux transporters have been described $(83,86-88)$.

Additionally, it was shown that increased $C D R 1$ expression could be induced in agents such as estradiol or fluphenazine (89). While it was previously shown that $C D R 1$ expression could be induced in response to fluconazole in a Cdr1-GFP fusion protein construct, a more recent study found that no increase in $C D R 1$ or $C D R 2$ expression as measured by RT-qPCR could be observed in the presence of fluconazole in a C. albicans strain with 6His3Flag-tagged Tac1 $(90,91)$. Both the constitutive and transient expression of $C D R 1$ and $C D R 2$ genes seems to be regulated via interactions at multiple cis-acting elements within the $C D R 1$ and $C D R 2$ promoters (92). The presence of drugresponsive elements (DRE) within each gene's respective promoter regions, for example, has been shown to contribute to the induced and constitutive expression of CDR1 and $C D R 2$, while a separate basal expression element (BEE) contributes to $C D R 1$ baseline expression $(82,89,93)$. 
In a genome-wide location analysis of Tac1 binding sites using ChIP-chip, Tac1 was shown to bind to the DRE of both $C D R 1$ and $C D R 2$ under nonactivating conditions, indicating that post-binding mechanisms such as repressor interaction loss or coactivator recruitment may occur during Tac1 activation (93). More recently, components of the tail module of the Mediator complex have been identified as required for Tac1-activated $C D R 1$ expression. In a study examining the coactivator requirements for hyperactive Tac1 regulation, Liu and Myers described decreased fluconazole MICs in TAC1 gain-offunction mutant strains lacking either Med3 or Med15, both of which are part of the Mediator tail module (91). Furthermore, not only was Tac1-driven transcription of CDR1 decreased in gain-of-function Tac1 mutant strains lacking either Med3 or Med15, but deletion of $M E D 3$ also diminished xenobiotic-induced expression levels of $C D R 1, C D R 2$, and $R T A 3$ with fluphenazine and estradiol. ChIP assays revealed that occupancy of the Mediator tail module at the CDRI DRE increases during Tac1 hyperactivation, as well as with $T A C 1$ overexpression, however higher occupancy at the DRE did not result in increased fluconazole MICs, unless Tac1 was activated. Importantly, the authors also delineated a C-terminal region of Tac1 (amino acids 865 to 981), so called the transcription activation domain (TAD), whose interaction with an as yet uncharacterized inhibitory middle domain, may be crucial to Tacl hyperactivation, and have posited that gain-of-function mutations in Tacl may work to antagonize the normally inhibitory interaction between these two regions (91).

\section{Mdr1}

Mdr1, part of the major facilitator superfamily of transporters, has also been shown to play a role in fluconazole and voriconazole resistance, though it does not effect large MIC changes with other azole antifungals such as itraconazole, isavuconazole, and posaconazole (94-96). The gene encoding the Mdr1 efflux pump in C. albicans was originally discovered by its ability to confer resistance to several structurally-unrelated compounds such as the anti-mitotic drug benomyl, methotrexate, cycloheximide, sulfometuron methyl, 4-nitroquinoline- $N$-oxide, and bentriazoles in Saccharomyces cerevisiae expressing the $C$. albicans $M D R l$ gene, which at the time was known as $B E N r$ $(97,98)$. It was not until serial isolates taken from HIV-infected patients with oropharyngeal candidiasis were analyzed for ERG11 and MDR1 mRNA expression levels was a link established between the $M D R 1$ gene and fluconazole resistance (99).

A study assessing sequential isolates of $C$. albicans in patients with recurrent oropharyngeal candidiasis treated with fluconazole found that increased $M D R 1$ mRNA, as well as increased $C D R 1 / 2$ and $E R G 11$ gene expression, correlated with increasing fluconazole drug resistance, indicating that $M D R 1$ plays a role in the multifaceted development of resistance in C. albicans (100). When the MDR I gene was disrupted in two fluconazole-resistant clinical $C$. albicans isolates, susceptibility increased to fluconazole compared to the resistant isolates with intact $M D R 1$, demonstrating a direct relationship between $M D R 1$ and fluconazole resistance in clinical isolates (101). 
Importantly, matched pairs of fluconazole-susceptible and fluconazole-resistant isolates were shown to lack major polymorphic differences in the nucleotide sequence of their respective $M D R 1$ promoter regions, despite high expression of MDRI mRNA in the fluconazole-resistance isolate (102). Subsequent expression of GFP from the MDRI promoter in the fluconazole-resistant isolate but not the fluconazole-susceptible isolate indicated that $M D R 1$ expression was likely guided by a trans-regulatory factor. Induction of MDRI mRNA expression through benomyl and tert-butyl hydrogen peroxide identified several cis-acting elements in the MDRl promoter involved in induction with these agents, though fluconazole itself failed to induce the MDRI promoter (103).

It was not until more recently in 2007 that the zinc-cluster transcription factor Mrr1 was discovered to regulate the Mdr1 efflux pump. Transcriptional profiling of three $M D R 1$-overexpressing clinical isolates via DNA microarray analysis revealed 21 genes coordinately upregulated with $M D R I$ and seven genes downregulated in all three isolates (104). Among these, the then putative zinc cluster transcription factor Zcf36, later known as the multidrug resistance regulator Mrr1, was hypothesized to control MDR1 expression. Deletion of MRR1 in MDR1-overexpressing isolates F5 and G5 managed to abolish $M D R 1$ promoter activity in a $\mathrm{P}_{M D R I}-G F P$ reporter fusion, implicating Mrrl in the constitutive expression of $M D R I$ in these fluconazole-resistant clinical isolates. Interestingly, deletion of $M R R I$ in each $M D R 1$-overexpressing isolate did not yield identical MICs to strains lacking MDRI in the same background. Deletion of MDRI alone in both cases, resulted in a higher MIC in the resistant clinical isolate background compared to deletion of its transcriptional regulator Mrr1, providing evidence that Mrr1 may regulate other determinants of fluconazole resistance apart from Mdr1.

Similar to the zinc cluster transcription factor encoding gene $T A C 1$ in $C D R 1$ - and $C D R 2$-overexpressing isolates, mutated $M R R 1$ alleles were present in the $M D R 1$ overexpressing clinical isolates (104). Moreover, sequencing of the $M R R I$ alleles in the $M D R 1$-overexpressing isolates F5 and G5 revealed a loss of heterozygosity and retention of two copies of a mutant MRRI allele in both clinical isolates. Replacement of a single copy of the mutant MRR1 allele encoding either a P683S or G997V amino acid substitution into SC5314 lacking both native copies of MRR1 resulted in increased resistance to fluconazole and increased $M D R 1$ promoter activity as measured by a $\mathrm{P}_{M D R 1^{-}}$ GFP reporter construct, demonstrating the presence of gain-of-function mutations in $M R R 1$ that regulate MDR1 expression in C. albicans.

Indeed, multiple amino acid substitutions in Mrrl originating from both human clinical isolates and in vitro generated strains have been shown to be gain-of-function mutations driving constitutive MDRl expression (105). Furthermore, through DNA sequence analysis of a the F1 through F5 matched series clinical isolates, it was revealed that gain-of function mutations appear to occur in a single $M R R 1$ allele initially, which provides an intermediate level of resistance compared to the matched isolates collected chronologically later in the series that were homozygous for the $M R R I^{\mathrm{P} 883 \mathrm{~S}}$ allele. Through analysis of marker polymorphisms along chromosome 3 distal to MRRI and proximal to the telomere, Dunkel et al. revealed that loss-of-heterozygosity with selection 
of mutated MRR1 alleles was accomplished through mitotic recombination in clinical isolates, though chromosome loss was also observed as a cause in MDR1 overexpressing strains developed in vitro.

\section{Upc2 and increased $E R G 11$ expression}

Upc2 is another $\mathrm{Zn}(\mathrm{II})-\mathrm{Cys}(6)$ transcription factor involved in sterol biosynthesis and azole resistance. Initially identified as a homolog of the $S$. cerevisiae (ScUPC2) gene, $U P C 2$ in C. albicans was initially reported to upregulate the ergosterol biosynthesis genes $E R G 2$ and $E R G 11$ when exposed to fluconazole (106). The overexpression of $E R G 11$ had been shown to contribute to azole through increased production of the azole target $14 \alpha$-lanosterol demethylase $(80,99,107,108)$. However, not only was the induction of ERG11 abolished when UPC2 was deleted, but the deletion strain was also hypersusceptible to the azole drugs as well as the squalene epoxidase (Erg1) target drug terbinafine, the Erg2 and Erg24 target drug fenpropimorph, and the HMG-CoA reductase inhibitor lovastatin, illustrating UPC2's important role in sterol biosynthesis regulation $(106,108)$.

Importantly, overexpression of Upc2 via expression of the ORF from the MET3 promoter increased resistance to fluconazole and ketoconazole, and it was also demonstrated for the first time that the DNA-binding domain of Upc2 bound directly to ergosterol biosynthesis genes, binding to a putative sterol responsive element in ERG2 (108). Furthermore, investigation of the ERG11 promoter through deletion and linker scan mutations concluded that two 7-bp inverted repeat sequences located -231 and -251 bp upstream of the start codon form an azole-responsive element (ARE) regulated by Upc2, and that this ARE was sufficient to induce ERG11 expression (109).

Upc2, however, was also shown to have a regulatory role in more than just sterol biosynthesis genes. Through genome-wide gene expression profiling, several ergosterol biosynthesis genes, including ERG11, were determined to be coordinately upregulated with $U P C 2$ in fluconazole resistant isolates, and ChIP-microarray location analysis identified 202 promoters bound by Upc2, including not only ergosterol biosynthesis genes (NCP1, ERG11, ERG1, ERG2, ERG24, ERG4, ERG5, ERG6, ERG9, ERG10, $E R G 25, E R G 251, U P C 2)$ but also other transcription factors and the drug transporter genes $C D R 1$ and $M D R 1$ (110-112). As with the zinc cluster transcription factors Tac1 and Mrr1, gain-of-function mutations in Upc2 were found to lead to increased gene expression of ERG11 conferring fluconazole-resistance in clinical isolates $(110,113$ 115). When $U P C 2$ was disrupted, the $\triangle u p c 2$ mutants had decreased cellular ergosterol content and were more susceptible to fluconazole not only in the azole-susceptible SC5314 strain but also in a highly resistant clinical isolate 12-99 which overexpresses $C D R 1, M D R 1, E R G 11$ and possesses a mutation in ERG11, suggesting that UPC2 is required for clinical azole drug resistance $(115,116)$. 


\section{Other zinc cluster transcription factors}

Apart from the zinc cluster transcription factors Tac1, Mrr1, and Upc2, which are well-known players in C. albicans azole resistance, there has been significant interest in the azole-resistance role of the remaining zinc-cluster transcription factors as well. In 2013, Morschhäuser et al. demonstrated that laboratory strains exhibiting hyperactive transcription factors Aro80, Mrr2, Stb5, Cta4, Zcf25, Zcf35, and Znc1 also displayed increased azole resistance comparable to hyperactive Tac1, Mrr1, and Upc2, demonstrating that in theory other ZCFs may play a role in azole resistance in C. albicans (117). Additionally, in the hyperactive ZCF strains, Znc1 and Mrr2 appeared to activate the CDR 1 promoter, indicating that these ZCFs may contribute to increased efflux pump expression.

In 2015, Wang et al. described the first clinical isolate containing putative gainof-function mutations in the ZCF Mrr2, identifying amino acid substitutions that appeared increased $C D R 1$, but not $C D R 2$ expression (118). In contrast to this finding, a recent report investigating these mutations in $M R R 2$ when inserted into the native locus as well as when the gene is overexpressed failed to identify any changes in fluconazole susceptibility or CDRI expression compared to wild type (119). Given the contradictory findings, the clinical impact of other ZCFs outside of Tac1, Mrr1, and Upc2 in azole resistance remains in question.

\section{Mutations in ERG11}

Mutations in the ERG11 gene itself is another common azole resistance mechanism in C. albicans, in stark contrast with the haploid organism C. glabrata, in which the frequency of mutations in ERG11 appears relatively low $(14,120)$. Most ERG11 mutations are thought to alter the ability of the azoles to bind and inhibit the lanosterol demethylase enzyme, either through steric hindrance in the ligand binding pocket where the azole antifungals compete with ergosterol precursor or by altering or reducing important interactions, such as the H-bond interactions with the azole ring and the heme group of the Erg11 protein.

The earliest point mutation resulting in an amino acid substitution (R467K) in the ERG11 gene of a clinical isolate was sequenced and discovered in 1997 (121). Soon after, multiple additional amino acid substitutions in Erg11 were uncovered, and notably, the effect of these amino acid changes on triazole antifungal MICs were not uniform (122). This altered susceptibility to the azoles was noted to likely be a result of altered interaction with azole binding and overall reduced fluconazole binding affinity to the mutant $14 \alpha$-lanosterol demethylase target enzyme $(123,124)$. As the azole antifungals emerged as a common, first-line option in the treatment of many superficial and invasive fungal infections, documentation of new ERG11 mutations in azole-resistant clinical isolates continued to appear, though not all documented mutations were definitively shown to be tied to azole resistance (125-138). 
The isolated contribution towards azole-resistance of several predominant Erg11 amino acid substitutions found in clinical $C$. albicans isolates was investigated through homozygous replacement of the native ERG11 alleles with a mutant ERG11 ORF encoding either a single or double amino acid substitution in the Erg11 protein (139). Single substitutions in Erg11 showed variable responses to fluconazole and other triazoles ranging from no effect to a 16-fold increase in MIC over the fluconazolesusceptible parent, and double substitutions in Erg11 further increased the MIC beyond the individual contribution to azole resistance of any single amino acid substitution. Interestingly, while some substitutions, such as Y132F/H, K143R, G307S, and S405F, are located on the exposed active-site cavity of the Erg11 protein and presumably directly influence or interfere with azole binding, other substitutions not predicted to directly interact with azole binding, such as the G450E substitution found on the fungal CYP51 specific $\beta 5$-hairpin, may indirectly affect resistance through interaction with cytochrome P450 reductase-Erg11 interactions (140).

Recently substitutions in purified, recombinant C. albicans Erg11 expressed from E. coli revealed that Erg11 variants differ substantially in their baseline catalytic turnover and affinity for azole binding (141). Despite some substitutions showing relatively loose azole binding, their observed contribution to azole resistance in in vitro C. albicans susceptibility testing was significant; making it conceivable that Erg11 amino acid substitutions may contribute to azole resistance through more nuanced ways than simply reducing azole binding affinity.

\section{Mutations in ERG3 and alternative sterol biosynthesis}

Alterations in sterol biosynthesis pathways in C. albicans remains an important, albeit rare clinical mechanism of azole antifungal resistance. Defective C5-6 desaturase, homologous to the Erg3 enzyme C-5 sterol desaturase, identified in azole-resistant isolates of Saccharomyces cerevisiae initially led to the currently hypothesized mode of action of the azole antifungals.

$14 \alpha$-methylergosta-8,24(28)-dien-3 $\beta, 6 \alpha$-diol, a metabolic byproduct not normally produced in significant amounts during normal ergosterol biosynthesis, was found to accumulate in $C$. albicans cells following treatment with fluconazole (72). However, isolates with defective C-5 sterol desaturase, which lacked detectable amounts of ergosterol presumably because they no longer possessed the $\operatorname{Erg} 3$ enzyme function required for ergosterol production, avoided accumulation of 14 $\alpha$-methylergosta-8,24(28)dien-3 $\beta, 6 \alpha$-diol after treatment with fluconazole. The continued growth of isolates with defective Erg3 in the presence of fluconazole has led to the current belief that $14 \alpha$ methylergosta-8,24(28)-dien-3 $\beta, 6 \alpha$-diol is toxic to the $C$. albicans cell, and its accumulation results in the inhibition of growth. Moreover, mutations and amino acid substitutions that confer defects in Erg3 also confer resistance to the azole antifungals, specifically due to the inability to produce $14 \alpha$-methylergosta-8,24(28)-dien-3 $\beta, 6 \alpha$-diol, which requires Erg3. 
While relatively few in number compared to isolates containing other more common resistance mechanisms, several mutant ERG3 clinical isolates have been identified, and reportedly possess resistance to the azole antifungals and cross-resistance to the polyene amphotericin B $(72,130,142-145)$. However, some question the clinical significance of $E R G 3$ mutant isolates during infection as isolates with an ERG3 resistance phenotype also displayed hyphal growth defects and attenuated virulence (146-148). Recently, it has been shown that changes within the ERG3 promoter that affect expression and activity may be sufficient to confer azole resistance in niche-specific instances without affecting $C$. albicans pathogenicity, which implicates changes in the $E R G 3$ locus as potentially more clinically relevant than previously believed (149).

\section{Aneuploidy and loss of heterozygosity}

Aneuploidy plays a role in azole resistance as well as genetic diversity in $C$. albicans. Gain or loss of chromosomes have been tied to azole resistance and attenuated virulence $(150,151)$. Loss of heterozygosity at gene loci of isolates serially passaged in azole-containing media as well as lab strains passaged through mice models of hematogenously disseminated disease both suggest that significant genetic rearrangements take place as a result of host and drug stress $(152,153)$. Studies using comparative genome hybridization in various $C$. albicans laboratory strains observed aneuploidies mainly on chromosomes 1,2 , and 5 (154), and it was later shown that some genomic changes such as isochromosome formation confer azole resistance in $C$. albicans (155).

Overrepresentation of efflux pumps, ERG11, or zinc cluster transcription factor genes involved in azole resistance as a result of chromosomal nondisjunctions, trisomy, or isochromosome formation is very likely to contribute to decreased drug resistance in these cells. Indeed, it had been shown for both TAC1 and MRR1 that loss of heterozygosity was responsible in some clinical isolates for homozygous gain-of-function mutations conferring increased efflux pump expression and azole resistance $(83,105)$. A recent analysis of large-scale genomic changes in 43 clinical isolates of $C$. albicans showed that while loss of heterozygosity events are persistent and often associated with resistance, aneuploidies in clinical isolates are often transient and did not correlate with drug resistance (156). The discovery of tetraploid cells of C. albicans, containing four homologous sets of chromosomes, and their ability to generate a diverse range of fitness and heterogeneity through increased rates of heterozygosity loss suggests that aneuploidy plays an important role in adaptive fitness to selective pressures under these circumstances $(157,158)$. The recent investigations into trisomy of chromosome $\mathrm{R}$ and chromosome 4 possibly conferring azole resistance in two different clinical isolates of $C$. albicans also underscores the importance of aneuploidy in the microevolution of drugresistant C. albicans $(159,160)$. 


\section{Azole import}

Currently there is limited evidence suggesting that reduced azole uptake has any clinical relevance to azole resistance in C. albicans or any other Candida species. However, it should be mentioned that it is plausible that reduced azole import could be a potential uncharacterized mechanism of drug resistance. In a study examining the in vitro accumulation of fluconazole into the Candida cell, it was observed that fluconazole import displayed saturation kinetics in cells de-energized with 2-deoxy-D-glucose and therefore unable to efflux drug via active transport mechanisms like the ABC transporter Cdr1 (161). Additionally, maximal accumulation of fluconazole did not progress in a temperature-dependent manner inconsistent with simple passive diffusion. The kinetics of fluconazole import, therefore, suggests that fluconazole is uptaken by means of a carrier protein via facilitated diffusion and that this mechanism is likely conserved across many fungal species $(161,162)$.

The carrier protein(s) responsible to importing fluconazole and presumably the other azoles, however, has/have not yet been identified despite prolific research. In theory, cells possessing defective carrier protein (via mutation, for example) or protein in low amounts would not accumulate lethal or significantly inhibitory amounts of drug, resulting in resistance.

\section{Azole tolerance}

Candida albicans is usually classified as either susceptible (and susceptible dosedependent) or resistant when referring to clinical antifungal breakpoints, which are used in helping to predict response to therapy against fluconazole or other antifungal agents $(29,33,163-165)$. Antifungal "tolerance" is an oft-used and loosely-defined term that refers generally to the residual growth of cells at or above inhibitory concentrations of drug.

Unlike resistant cells, which may be able to survive drug treatment due to genedependent mechanisms directly affecting the drug, drug target, or accumulation of the drug in the cell, cells displaying tolerance have been defined as those able to survive transient exposure to drug without an accompanying change in MIC (166-168). The "trailing growth" phenomenon, describing the residual population of cells that grow at supra-MIC levels observed in broth dilution assays, can be considered related to azole tolerance and has been well-documented in C. albicans (168-171). Recently, RTA3, encoding a putative lipid translocase coordinately upregulated with $C D R 1$ in clinical isolates with Tac1 gain-of-function mutations, has been associated with increased azole tolerance and trailing growth in C. albicans (172).

The presence and degree of trailing growth has been known to be affected by different parameters such as temperature and $\mathrm{pH}$ and can affect the MIC interpretation depending on the time of reading (24 hours versus 48 hours) $(170,173,174)$. However, the clinical significance of trailing growth has been dubious at best. Past literature 
suggests that the $C$. albicans isolates exhibiting trailing growth phenomena responds to azole treatment similarly to susceptible isolates in murine models and in a small sample size of HIV-infected patients with recurrent oropharyngeal candidiasis (175-177).

Importantly, a recent study delineated parameters to describe the antifungal tolerance observed with certain strains. The "fraction of growth," or FoG, measured on antifungal disk diffusion assay and the "supra-MIC growth," or SMG, as measured by broth microdilution assay are objective measurements assessing growth either inside the zone of inhibition or above the MIC for a given strain, respectively (178). Neither parameter correlated with MIC and instead measured a drug response distinct from MIC. Interestingly, FoG and SMG were independent of inoculum size, cell density or drug concentration, and in contrast to tolerance in bacteria, tolerant $C$. albicans cells did not have reduced growth rates. Additionally, FoG did not correlate with growth rate or colony size, indicating that high levels of FoG are not due simply to faster cell growth. Instead, $C$. albicans isolates with high levels of FoG or SMG suggests a larger subpopulation that can respond to high levels of antifungal drug stress better than isolates with low FoG or SMG.

When comparing the drug responses of clinical isolates taken from patients with persistent candidemia versus patients with candidemia that was cleared after a single treatment course of fluconazole, significantly higher FoG and SMG levels was observed in clinical isolates originating in patients with persistent candidemia (178). This was despite the fact that the tested isolates in both groups all had in vitro MICs considered fluconazole-susceptible, and identified FoG and SMG as potential parameters to guide treatment outcome in patients with candidemia. This tied with the identification of several adjuvant drugs that could reduce FoG but not MIC in clinical isolates suggests that drug tolerance in $C$. albicans may be an overlooked issue of significant clinical relevance.

\section{Future Directions}

As we have discussed, $C$. albicans has an arsenal of defense mechanisms that it uses, alone and in combination with each other, to overcome inhibition by the azole antifungals. Despite the numerous resistance pathways already discovered, continued investigation is required to fully characterize azole resistance in C. albicans. For example, the azole importer(s) in Candida and other fungal species has still not been identified, and its discovery would plausibly reshape our approach to overcoming azole resistance.

Ongoing research is also identifying potential novel mechanisms of azole resistance, though the clinical significance of these are still unknown. Recently, a Nature Communications paper published that alteration of sphingolipid synthesis mediated through FEN1 and FEN12 deletion results in fluconazole resistance, and these composition changes are similar to what is observed in wildtype cells treated with fluconazole (179). Furthermore, transposon-based mutagenesis and disruption of the $C$. 
albicans genome in a haploid strain identified a number of potential genes involved in drug stress response through Gene Set Enrichment Analysis, providing further insight into C. albicans acquisition of drug resistance.

As more of the machinery driving azole resistance becomes unveiled, we can begin to isolate the cogs critical to its function. Targeting these components with novel drug development and improved use of our existing armamentarium becomes the immediate goal in addressing the rising problem of azole resistance and antifungal drug resistance in general.

\section{Hypothesis and Objectives}

Although C. albicans is a commensal organism that is part of normal flora in the majority of the human population (2), it is an opportunistic pathogen that has been the major infective species of Candida found in invasive candidiasis $(17,180,181)$. For invasive fungal infections like Candida bloodstream infections, mortality rates remain high $(182,183)$. Fluconazole and other members of the azole class continue to be commonly used in the treatment of Candida infections, and overall have good activity against a majority of $C$. albicans isolates (15). However, azole-resistant Candida is of serious concern, especially in those patient with prior exposure to the azole antifungals (184-186). The ultimate, overarching goal of my research has been to improve the health outcomes of patients treated with the azoles for $C$. albicans infections. By investigating mechanisms of azole resistance in $C$. albicans, I hope to identify novel determinants in $C$. albicans that may prove useful either in the determination and detection of clinical isolates predisposed to azole non-susceptibility or as a drug target in the development of new antifungals.

In previous work performed by our research group, the major known resistance determinants, including the expression of the efflux pump encoding genes $C D R 1, C D R 2$, and $M D R 1$ as well as mutations in and increased expression of ERG11, encoding the azole target $14 \alpha$-lanosterol demethylase, were identified and characterized in a large collection of predominantly fluconazole-resistant clinical isolates $(115,116)$. Here it was revealed that some clinical isolates possessed higher fluconazole MICs than what could be reasonably justified based on the known mechanisms of azole resistance (Figure 1-1). Hence, I had reason to further investigate these clinical isolates for hidden contributors of azole resistance.

Previous literature supports the hypothesis that other mechanisms of azole resistance remain uncharacterized in clinical isolates. The critical role of the zinc cluster transcription factors Tac1, Mrr1, and Upc2 in regulating gene expression of azole resistance determinants led to identification of several other zinc cluster transcription factors in C. albicans that can influence azole susceptibility in laboratory strains (117). Through sequencing of the ZCF encoding gene $M R R 2$, I identified several novel mutations in our clinical isolates. A recent publication suggested that this $\mathrm{ZCF}$ is important in the regulation of CDRl expression in clinical isolates (118). In some of our 


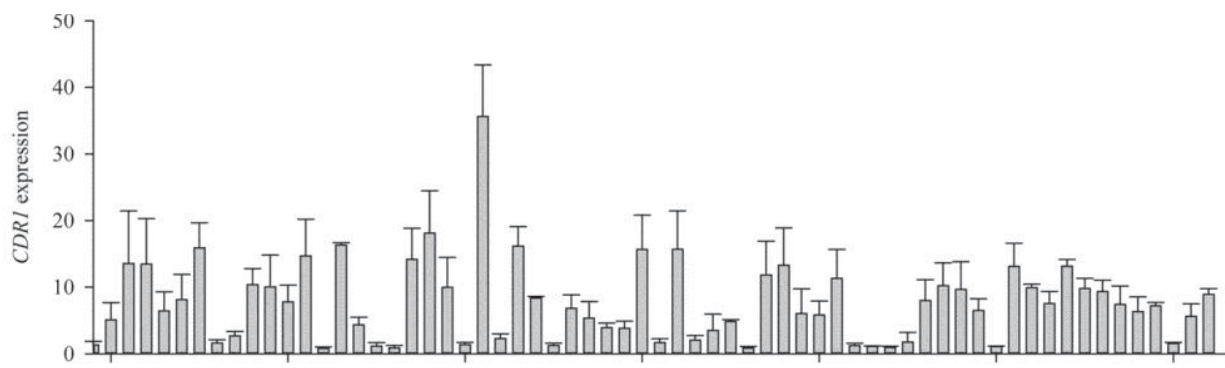

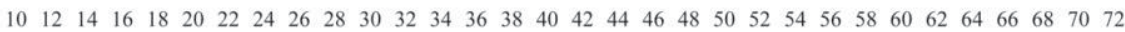

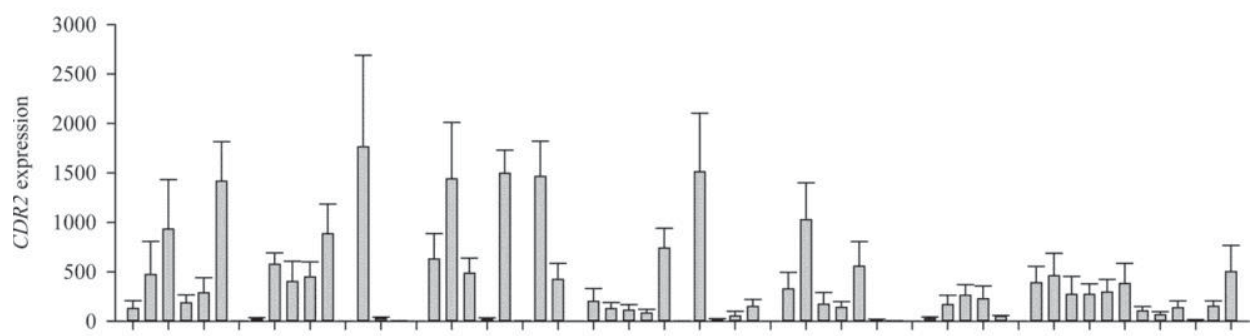

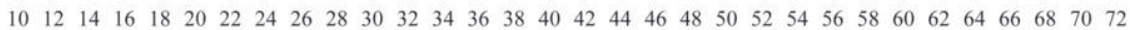

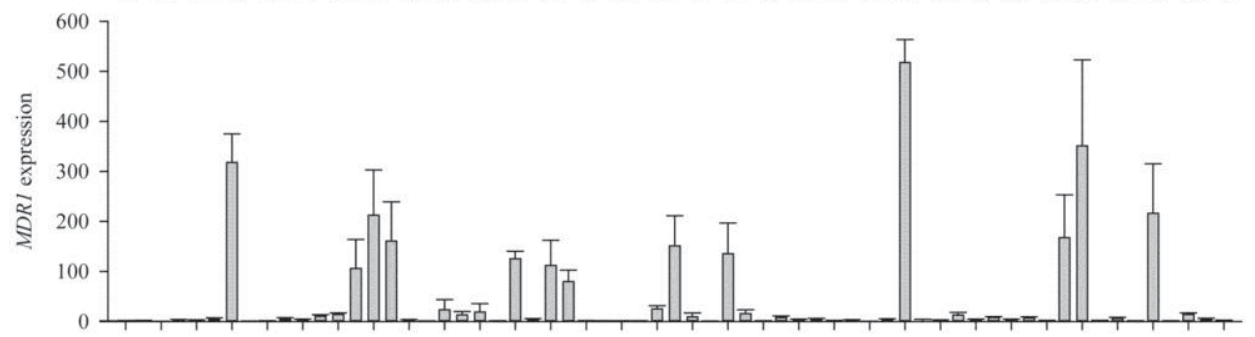

$10121416182022242628 \quad 3032 \quad 34 \quad 36 \quad 3840 \quad 4244 \quad 4648505254565860626466 \quad 68 \quad 7072$

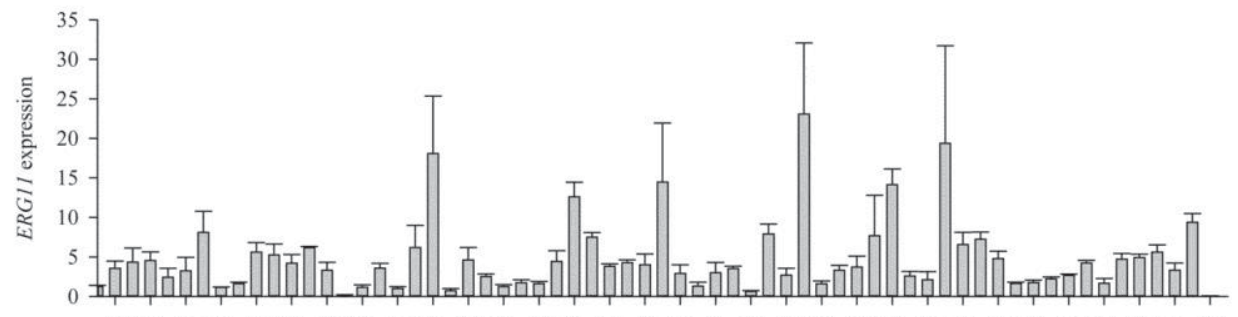

$\begin{array}{llllllllllllllllllllllllllllllll}10 & 12 & 14 & 16 & 18 & 20 & 22 & 24 & 26 & 28 & 30 & 32 & 34 & 36 & 38 & 40 & 42 & 44 & 46 & 48 & 50 & 52 & 54 & 56 & 58 & 60 & 62 & 64 & 66 & 68 & 70 & 72\end{array}$

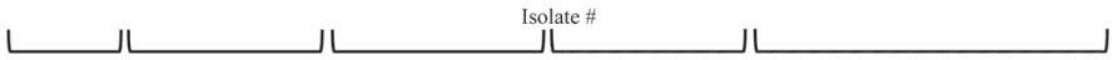

$\begin{array}{llllll}\text { MIC } & 16 & 32 & 64 & 128 & 256\end{array}$

Figure 1-1. Expression levels of $C D R 1, C D R 2, M D R 1$, and $E R G 11$ in 63 fluconazole-resistant clinical isolates

All gene expression levels were measured in triplicate, and fold expression of genes in resistant isolates was compared to the average of the expression levels in three susceptible isolates. Results for 63 isolates with reduced susceptibility to fluconazole are represented, but only even-numbered isolates' results are labeled. Error bars show standard errors. Reprinted from final submission with permission from the American Society for Microbiology. Flowers SA, Barker KS, Berkow EL, Toner G, Chadwick SG, Gygax SE, Morschhauser J, Rogers PD. 2012. Gain-of-function mutations in UPC2 are a frequent cause of ERG11 upregulation in azole-resistant clinical isolates of Candida albicans. Eukaryot Cell 11:1289-99. doi: 10.1128/EC.00215-12. 
isolates, $C D R 1$ expression was increased without a corresponding gain-of-function mutation in $T A C 1$. Therefore, I hypothesized that gain-of-function mutations in MRR2 was responsible for increased CDR1 expression and increased fluconazole MICs in some of our clinical isolates. My summarized aim was to characterize these mutations with respect to their effect on fluconazole susceptibility and $C D R 1$ expression by placing the mutation-containing $M R R 2$ alleles in a fluconazole-susceptible clinical isolate.

Previously, our research group had defined the contributions of individual and combination amino acid substitutions in Erg11 (CaCYP51) to azole resistance in $C$. albicans (139). Amino acid substitutions in CaCYP51 are a well-established resistance mechanism that $C$. albicans possesses to reduce the ability of the azoles to inhibit CaCYP51 activity. The effects of these amino acid substitutions on CaCYP51 function and cell fitness, however, had not been previously studied. In a collaborative effort, our research group aimed to determine the differences in biochemical function and interaction between mutant CaCYP51 proteins, and whether this translates to reduced in vitro azole susceptibility in $C$. albicans cells. We hypothesized that mutant CaCYP51 protein have variable binding affinities with the azole antifungals, affecting azole susceptibility. Furthermore, we believed that amino acid substitutions not only affect CaCYP51's interaction with the azoles but also CaCYP51 native activity. My goal was to create additional mutant CaCYP51 C. albicans strains and evaluate the in vitro susceptibility data as compared to the biochemical data in this project.

Mutations in ERG3, a gene encoding the C-5 sterol desaturase important for ergosterol and toxic sterol production, are an oft overlooked mechanism of azole resistance due to its relative scarcity in clinical isolates. As part of the investigation into azole resistance within our clinical isolates, I also sequenced the $E R G 3$ gene in our collection. Interestingly, in addition to a nonsense mutation in $E R G 3$ in one clinical isolate with altered sterol composition, I frequently sequenced an uncharacterized A351V Erg3 amino acid substitution. Although I found no consistent correlation between ergosterol content and $E R G 3$ mutations, I noticed a high coincidence of $E R G 3$ mutations with ERG11 mutations. I posited that amino acid substitutions in Erg3, such as the A351 V mutations may play an indirect role in azole resistance by conferring either a fitness advantage to isolates with ERG11 mutations. Preliminary data detailed in our research group's collaborative work with Erg11 mutants suggested that in addition to conferring varying changes in azole susceptibility, Erg11 amino acid substitutions also conferred changes in the Erg11 enzyme's functional efficiency and, therefore, also to the fitness of the cell. My hypothesis was that the A351V amino acid substitution in Erg3 was a permissive substitution that compensated for changes in Erg11 activity resulting from deleterious amino acid substitutions. My initial aim was to determine whether the A351V amino acid substitution conferred any advantage to cells also containing ERG11 mutations.

Lastly, the novel investigational drugs VT-1161 and VT-1598 had been previously shown to have good activity against Candida and a wide variety of other fungi (187-189). However, C. albicans mechanisms of resistance against these two agents have not been clearly defined. Given our collection of azole-resistant clinical isolates and 
laboratory strains with defined mechanisms of azole resistance, our research group endeavored to analyze the susceptibility data of our strains and isolates against both drugs. The goals here were to identify known mechanisms of azole resistance that may affect drug susceptibility to either VT-1161 or VT-1598 and to identify clinical isolates that possess increased MICs to the tetrazoles. Identifying mechanisms affecting tetrazole susceptibility would be useful in determining the role of either VT-1161 or VT-1598 as prospective therapeutic agents in invasive Candida infection, especially in resistant or recurrent infection. Additionally, given that our collection of clinical isolates contained isolates with unusually high azole MICs that could not be explained, we hoped that the additional tetrazole susceptibility data might provide insight on their resistance. 


\section{CHAPTER 2. THE CONTRIBUTION OF CLINICALLY-DERIVED MUTATIONS IN THE GENE ENCODING THE ZINC CLUSTER TRANSCRIPTION FACTOR MRR2 TO FLUCONAZOLE ANTIFUNGAL RESISTANCE AND CDR1 EXPRESSION IN CANDIDA ALBICANS ${ }^{1}$}

\section{Introduction}

Candida spp. account for one of the most common pathogen groups implicated in nosocomial bloodstream infections in the US $(12,183)$. Candida albicans is the most common Candida spp. in invasive candidiasis and the primary cause of mucosal Candida infections such as oropharyngeal candidiasis $(34,70,183,190,191)$. Fluconazole and other azoles are a mainstay of treatment in invasive candidiasis and oropharyngeal candidiasis, and despite the emergence of other antifungal drug classes, there has been no substantial decline in fluconazole resistance rates in $C$. albicans $(29,30,34,192)$. Moreover, the high mortality rate for $C$. albicans in invasive fungal disease provides compelling reason to further investigate how we can better utilize the available antifungal drugs in order to overcome treatment failure $(190,193,194)$. Therefore, it is imperative that we achieve a comprehensive understanding of the mechanisms of azole resistance within this organism.

Current understanding of fluconazole resistance within C. albicans involves sterol biosynthesis gene mutations as well as zinc-cluster transcription factor-mediated changes in efflux pump activity and drug target abundance (14). Zinc-binding proteins play a crucial role in the transcriptional regulation of a vast number of genes in eukaryotes, and in particular, the zinc cluster transcription factors (ZCFs) within the fungal kingdom make up one of the largest regulatory protein families in yeast $(117,195)$. Regarding azole antifungal resistance in Candida spp., ZCFs play a major role in almost all species where azole resistance mechanisms have been elucidated, including $C$. glabrata and $C$. parapsilosis (14). Changes in the zinc-cluster transcription factors Tac1, Mrr1, and Upc2 are thought to be responsible for the bulk of fluconazole resistance in $C$. albicans $(14,78$, $79,83,104,113,115,116,196)$. The presence of gain-of-function mutations in the transcription factor genes $T A C 1$ and $M R R 1$ results in increased expression of the efflux pump genes $C D R 1 / C D R 2$ and $M D R 1$, respectively, while gain-of-function mutations discovered in UPC2 lead to overexpression of the gene encoding the azole target, Erg11 (14 $\alpha$-sterol demethylase) $(83,104,113)$. Other mechanisms of azole resistance include changes in the azole target $14 \alpha$-sterol demethylase through mutations in its encoding gene ERG11 and less commonly through mutations in the sterol-C5(6)-desaturase encoding gene ERG3 $(71,121,122,142)$.

There is evidence strongly suggesting that other mechanisms of azole resistance

\footnotetext{
${ }^{1}$ Reprinted from final submission with permission. Nishimoto AT, Zhang Q, Hazlett B, Morschhäuser, Rogers PD. (2019). The contribution of clinically-derived mutations in the gene encoding the zinc cluster transcription factor Mrr2 to fluconazole antifungal resistance and CDR1 expression in Candida albicans. Antimicrob Agents Chemother. doi:10.1128/aac.00078-19:AAC.00078-19.
} 
in Candida spp. exist. For example, it has been previously demonstrated that other ZCFs can alter susceptibility to fluconazole when transcriptionally activated (117). In particular, decreased fluconazole susceptibility was observed in a laboratory strain expressing artificially-activated Mrr2. The hyperactive ZCF Mrr2 was noted to activate the $C D R 1$ promoter but not the $C D R 2$ promoter, in contrast to activated Tac1, which is known to increase both $C D R 1$ and $C D R 2$ expression (82). Additionally, fluconazole sensitivity was restored after deletion of the CDR1 gene in the hyperactive Mrr2 strain (117). More recently, potential activating mutations in Mrr2 which increased expression of $C D R 1$ but not CDR2 have been described in fluconazole-resistant clinical isolates of C. albicans, indicating that not only can these mechanisms manifest in vitro, but also that they are clinically relevant (118). Mrr2 is a relatively understudied ZCF whose role in clinical azole resistance has not yet been completely defined, but the potential significance of Mrr2 as a contributing azole resistance determinant in C. albicans cannot be overlooked.

In the present study, we investigated the contributions to fluconazole resistance of mutations in MRR2 oberved among isolates within a collection of azole-resistant clinical C. albicans isolates. To accomplish this, we sequenced the $M R R 2$ open reading frame (ORF) in a collection of 57 clinical isolates with reduced susceptibility to fluconazole (MIC range: 4 to $>64 \mu \mathrm{g} / \mathrm{mL}$, data not shown). Screening of the strains revealed multiple polymorphisms in $M R R 2$, several of which had been previously described to impact fluconazole MICs. Clinically-derived MRR2 alleles were introduced in a fluconazolesusceptible background to generate strains containing multiple single nucleotide polymorphisms (SNPs) in the MRR2 gene. In contrast to a previous report (118), we found no changes in fluconazole MIC or $C D R I$ expression upon introduction of any of the MRR2 SNPs observed in clinical isolates in our collection or described previously.

\section{Results}

\section{SNPs in MRR2 among fluconazole-resistant clinical isolates of $C$. albicans}

To identify clinically-occurring amino acid substitutions in Mrr2, we sequenced the MRR2 gene of 67 C. albicans clinical isolates (10 fluconazole-susceptible, 57 fluconazole-resistant) that we have previously described $(115,139)$. This collection of isolates had been previously characterized for known resistance mechanisms and collectively possessed every known mechanism of azole resistance $(115,139)$. Overall, 25 (44\%) of 57 fluconazole-resistant isolates possessed SNPs resulting in amino acid changes in Mrr2 (Appendix A), however, many of these SNPs were also observed in fluconazole-susceptible isolates and would not be expected to influence fluconazole MICs. Seven amino acid substitutions resulting from SNPs in MRR2 were present only among fluconazole-resistant isolates within our collection. Three of these substitutions (R45Q, A459T, V486M) had not previously been described in the literature. The remaining four substitutions (S466L, A468G, S469T, T470N) had been previously found in resistant clinical C. albicans isolates and reported to have an effect on fluconazole 
MIC and CDRI expression (118). Within our collection, these four substitutions appeared in four clinical isolates and exclusively co-occurred with each other.

Interestingly, constitutive $C D R 1$ expression levels were less than two-fold greater than that of a composite of fluconazole-susceptible isolates in five clinical isolates containing either the A459T substitution or the combination substitutions of S466L, A468G, S469T, and T470N (Table 2-1).

\section{Fluconazole susceptibility in MRR2 mutant strains}

To determine if the newly discovered amino acid changes in Mrr2 affected fluconazole susceptibility, we introduced two copies of the MRR2 allele from fluconazole-resistant isolates $23,20,28$, and 29 containing respectively the R45Q, A459T, V486M, and combined amino acid substitutions S466L, A468G, S459T, and $\mathrm{T} 470 \mathrm{~N}$ into the $m r r 2 \Delta / \Delta$ strain (SCZCF34M4A) via homologous recombination using the $S A T 1$ flipper technique. This subsequently yielded homozygous mutant strains $M R R 2^{\mathrm{R} 45 \mathrm{Q}}$,

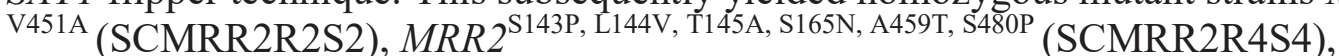
$M R R 2^{\text {T83A, A459T, S480P, } V 486 \mathrm{M}}$ (SCMRR2R5S5), and MRR2 $2^{\text {S466L, A468G, S469T, T470N, S480P }}$ (SCMRR2R6S6), which we hereafter will refer to as $M R R 2^{\mathrm{R} 45 \mathrm{Q}}, M R R 2^{\mathrm{A} 459 \mathrm{~T}}, M R R 2^{\mathrm{A} 459 \mathrm{~T}}$, ${ }^{\mathrm{V} 486 \mathrm{M}}$, and $M R R 2^{\mathrm{S} 466 \mathrm{~L}, \mathrm{~A} 468 \mathrm{G}, \mathrm{S} 469 \mathrm{~T}, \mathrm{T470N}}$, respectively. An additional mutant, $M R R 2^{\mathrm{T} 83 \mathrm{~A} \text {, }}$ V451A, V582L (SCMRR2R3S3), hereafter referred to as $M R R 2^{\mathrm{V} 582 \mathrm{~L}}$, was similarly constructed from the $M R R 2$ allele of fluconazole-susceptible isolate 22 as it contained a V582L substitution not previously described in the literature. For comparison, we created a $M R R 2^{\mathrm{WT}}$ (SCMRR2R1S1) strain by inserting wildtype $M R R 2$ amplified from fluconazole-susceptible clinical isolate SC5314 into the $m r r 2 \Delta / \Delta$ strain and included the previously described hyperactive Mrr2 strain (SCZCF34GAD1), which highly expresses artificially-activated Mrr2 (117). Surprisingly, none of the MRR2 mutant strains showed any change in fluconazole susceptibility as compared to SC5314 or $M R R^{\mathrm{WT}}$ as measured by CLSI standard methods (Table 2). This included $M R R 2^{\mathrm{S} 466 \mathrm{~L}, \mathrm{~A} 468 \mathrm{G}, \mathrm{S} 469 \mathrm{~T}, \mathrm{~T} 470 \mathrm{~N}}$, whose $M R R 2$ alleles contained four of six mutations previously reported to have an effect on fluconazole susceptibility. As expected, hyperactive Mrr2 exhibited elevated MICs to fluconazole. Strain susceptibilities measured on RPMI-agar using E-test strips also did not show reduced fluconazole susceptibility for any polymorphism-containing $M R R 2$ strain compared to SC5314. However, via E-test, we observed that the $m r r 2 \Delta / \Delta$ strain was slightly hypersusceptible to fluconazole compared to SC5314, as has been established previously (Table 2-2) (117).

\section{$M R R 2$ mutant strains do not overexpress $C D R 1$ when expressed from the $M R R 2$ locus}

To investigate whether any of the $M R R 2$ mutant strains could constitutively increase expression of the efflux pump gene $C D R 1$, we measured the $C D R 1$ mRNA abundance for each $M R R 2$ strain compared to SC5314 (Figure 1A) in triplicate via RTqPCR. Expression of CDRl was not significantly increased compared to SC5314 in any of the created strains containing polymorphic $M R R 2$ alleles. $M R R 2^{\mathrm{R} 45 \mathrm{Q}}, M R R 2^{\mathrm{A} 459 \mathrm{~T}}$, and 
Table 2-1. Mrr2 amino acid substitutions found in azole-resistant clinical isolates expressing $C D R 1$

\begin{tabular}{|c|c|c|c|}
\hline Mrr2 amino acid substitution & Isolate ID & $\begin{array}{c}24 \mathrm{H} \mathrm{MIC} \\
(\mu \mathrm{gL} / \mathrm{mL})\end{array}$ & $\begin{array}{l}\text { Fold } C D R 1 \\
\text { Expression }\end{array}$ \\
\hline V486M & 15 & 16 & 15.9 \\
\hline V486M & 27 & 16 & 14.2 \\
\hline V486M & 28 & 64 & 18.1 \\
\hline $\mathrm{A} 459 \mathrm{~T}$ & 18 & 32 & 10.4 \\
\hline $\mathrm{A} 459 \mathrm{~T}$ & 19 & 32 & 10.0 \\
\hline $\mathrm{A} 459 \mathrm{~T}$ & 20 & 64 & 7.8 \\
\hline $\mathrm{A} 459 \mathrm{~T}$ & 33 & 32 & 16.2 \\
\hline $\mathrm{A} 459 \mathrm{~T}$ & 41 & 64 & 1.7 \\
\hline $\mathrm{A} 459 \mathrm{~T}$ & 48 & 32 & 13.3 \\
\hline $\mathrm{A} 459 \mathrm{~T}$ & 54 & 32 & 0.9 \\
\hline $\mathrm{R} 45 \mathrm{Q}$ & 23 & 32 & 16.3 \\
\hline 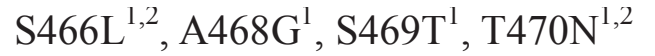 & 16 & 8 & 1.6 \\
\hline 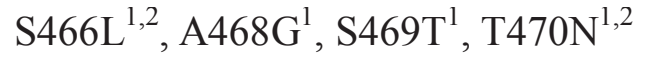 & 26 & 16 & 0.9 \\
\hline 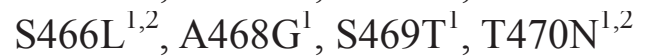 & 29 & $>64$ & 10 \\
\hline $\mathrm{S} 466 \mathrm{~L}^{1,2}, \mathrm{~A} 468 \mathrm{G}^{1}, \mathrm{~S} 469 \mathrm{~T}^{1}, \mathrm{~T} 470 \mathrm{~N}^{1,2}$ & 46 & 64 & 0.8 \\
\hline
\end{tabular}

${ }^{1}$ Amino acid substitutions previously reported to increase fluconazole MICs (118).

${ }^{2}$ Amino acid substitutions previously reported to increase $C D R 1$ expression at least twofold versus susceptible comparator (118). 
Table 2-2. Fluconazole susceptibilities for MRR2 mutant strains

\begin{tabular}{|c|c|c|c|}
\hline Strains & Strain description ${ }^{1}$ & $\begin{array}{l}\text { 24H FLU MIC } \\
(\mu \mathrm{g} / \mathrm{mL}, \text { CLSI) }\end{array}$ & $\begin{array}{l}\text { 24H FLU MIC } \\
\text { ( } \mu \mathrm{g} / \mathrm{mL}, \text { E-test) }\end{array}$ \\
\hline SC5314 & WT & 0.25 & 0.125 \\
\hline SCZCF34M4A & $m r r 2 \Delta / \Delta$ & 0.25 & 0.064 \\
\hline SCZCF34GAD1A & overexpressed $M R R 2-\mathrm{GAD}$ & 0.5 & 0.75 \\
\hline SCMRR2R1S1 & $M R R 2^{\mathrm{WT}}$ & 0.25 & 0.094 \\
\hline SCMRR2R2S2 & $M R R 2^{\mathrm{L} 143 \mathrm{P}, \mathrm{L} 144 \mathrm{~V}, \mathrm{~T} 145 \mathrm{~A}, \mathrm{~S} 165 \mathrm{~N}, \mathrm{~A} 459 \mathrm{~T}, \mathrm{~S} 480 \mathrm{P}}$ & 0.25 & 0.125 \\
\hline SCMRR2R3S3 & $M R R 2^{\mathrm{T} 83 \mathrm{~A}, \mathrm{~V} 451 \mathrm{~A}, \mathrm{~V} 582 \mathrm{~L}}$ & 0.25 & 0.125 \\
\hline SCMRR2R4S4 & $M R R 2^{\mathrm{R} 45 \mathrm{Q}, \mathrm{V} 451 \mathrm{~A}}$ & 0.25 & 0.125 \\
\hline SCMRR2R5S5 & $M R R 2^{\mathrm{T} 83 \mathrm{~A}, \mathrm{~A} 459 \mathrm{~T}, \mathrm{~S} 480 \mathrm{P}, \mathrm{V} 486 \mathrm{M}}$ & 0.25 & 0.094 \\
\hline SCMRR2R6S6 & $M R R 2^{\mathrm{S} 466 \mathrm{~L}, \mathrm{~A} 468 \mathrm{G}, \mathrm{S} 469 \mathrm{~T}, \mathrm{~T} 470 \mathrm{~N}, \mathrm{~S} 480 \mathrm{P}}$ & 0.25 & 0.094 \\
\hline SCMRR2R8S8 & $M R R 2^{C 9}$ & 0.25 & 0.125 \\
\hline SCMRR2GAD1R1S1 & $M R R 2-G A D$ & 0.5 & 0.19 \\
\hline $\mathrm{SC} \Delta \mathrm{mrr} 2 \mathrm{P}_{\mathrm{ADH} 1} \mathrm{MRR} 2^{\mathrm{WT}} \mathrm{A}$ & 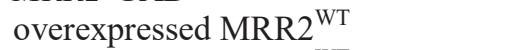 & 0.25 & 0.125 \\
\hline $\mathrm{SC} \Delta \mathrm{mrr} 2 \mathrm{P}_{\mathrm{ADH} 1} \mathrm{MRR} 2^{\mathrm{WT}} \mathrm{B}$ & overexpressed MRR2 $^{\mathrm{WT}}$ & 0.25 & 0.125 \\
\hline $\mathrm{SC} \Delta \mathrm{mrr} 2 \mathrm{P}_{\mathrm{ADH} 1} \mathrm{MRR} 2{ }^{\mathrm{C} 9} \mathrm{~A}$ & overexpressed MRR2 ${ }^{\mathrm{C} 9}$ & 0.25 & 0.125 \\
\hline $\mathrm{SC} \Delta \mathrm{mrr} 2 \mathrm{P}_{\mathrm{ADH} 1} \mathrm{MRR} 2^{\mathrm{C} 9} \mathrm{~B}$ & overexpressed MRR2 ${ }^{\mathrm{C} 9}$ & 0.25 & 0.125 \\
\hline
\end{tabular}

${ }^{1} \mathrm{GAD}=3 \mathrm{X}$ HA-tagged GAL4 activation domain 
$M R R 2^{\mathrm{A} 459 \mathrm{~T}, \mathrm{~V} 486 \mathrm{M}}$, which contained novel Mrr2 substitutions found only in fluconazoleresistant isolates, did not appear to express $C D R 1$ to a greater degree than $M R R 2^{\mathrm{WT}}$ or SC5314. MRR $2^{\text {S466L, A468G, S469T, T470N }}$, containing four mutations thought to influence $C D R 1$ expression also showed no increases over $M R R^{\mathrm{WT}}$ or SC5314, in line with our clinical isolates containing these substitutions in Mrr2 but not overexpressing CDR1. The strain expressing hyperactive Mrr2 showed an approximately eight-fold increase in $C D R 1$ over the control, consistent with its reduced susceptibility to fluconazole.

\section{Amino acid substitutions S466L, A468G, S469T, and T470N do not influence fluconazole MICs or CDR1 expression}

The four amino acid substitutions S466L, A468G, S469T, and T470N that were introduced into $M R R 2^{\mathrm{S} 466 \mathrm{~L}, \mathrm{~A} 468 \mathrm{G}, \mathrm{S} 469 \mathrm{~T} \text {, T470N }}$ failed to alter fluconazole susceptibility or change $C D R 1$ expression from SC5314. However, since we created this strain by inserting the entire $M R R 2$ allele taken from a resistant isolate, these substitutions were accompanied by other polymorphisms within the $M R R 2$ allele, and thus we could not rule out an interaction between these polymorphisms and the Mrr2 substitutions S466L, A468G, S469T, and T470N that could mask the increased fluconazole MICs previously associated with these substitutions. Therefore, we created the mutant $M R R^{\mathrm{S} 143 \mathrm{P}, \mathrm{L} 144 \mathrm{~V} \text {, }}$ T145A, H358N, E439K, V451A, S466L, A468G, S469T, T470N, S480P strain (SCMRR2R8S8, hereafter referred to as $M R R 2^{\mathrm{C} 9}$ ) by introducing a mutant $M R R 2$ allele containing 13 nucleotide changes corresponding to 11 amino acid substitutions in Mrr2 (Table 2-3) in order to verify the phenotype associated with these changes described in the literature (118). Surprisingly, $M R R 2^{\mathrm{C} 9}$ fluconazole MICs (Table 2-2) and $C D R 1$ expression (Figure 2-1A) were not different from SC5314, suggesting that these amino acid changes did not impact fluconazole resistance or $C D R 1$ expression when $M R R 2$ was expressed from the native promoter.

Our tested strains utilized homologous recombination to re-introduce polymorphic $M R R 2$ alleles back into the $m r r 2 \Delta / \Delta$ strain at the native $M R R 2$ locus. In order to substantiate our method of strain creation, we used the $S A T 1 /$ FLP strategy to introduce two copies of the $M R R 2$ allele in which the $\mathrm{C}$-terminal end of the gene was fused to the GAL4 activation domain and 3X HA-tagged, creating the mutant strain MRR2-GAL4AD3xHA (SCMRR2GAD1R1S1). Fusion of the HA-tagged Gal4 activation domain to fulllength ZCFs had been previously shown to constitutively activate fungal ZCFs such as Tac1, Upc2, and Mrr1 when expressed from the $A D H 1$ promoter (117). Here, our purpose was to create a constitutively active Mrr2 strain in which both copies of MRR2 were natively expressed. CDR1 expression increased $\sim 2.5$-fold from SC5314 in this strain, which resulted in slightly increased MICs to fluconazole over SC5314 as well, showing that homozygous replacement of the $M R R 2$ allele in the native locus using the $S A T 1 /$ FLP method was capable of producing changes in CDR1 expression and fluconazole MIC without foreign promoter-driven overexpression of $M R R 2$. This change was smaller than the increase in $C D R 1$ expression and fluconazole MIC seen with the hyperactivated $M R R 2$ strain, in which $M R R 2$ is expressed from the strong $A D H 1$ promoter. 
Table 2-3. Strains used in Chapter 2

\begin{tabular}{|c|c|c|}
\hline Strains & Genotype $^{1,2}$ & Source or reference \\
\hline SC5314 & Wildtype & ATCC \\
\hline SCZCF34M4 A/B & $\Delta m r r 2:: \mathrm{FRT} / \Delta m r r 2:: \mathrm{FRT}$ & $(117)$ \\
\hline SCZCF34GAD1 A/B & 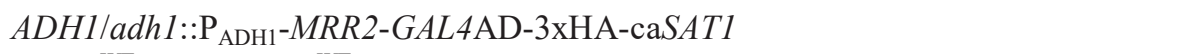 & $(117)$ \\
\hline SCMRR2R1S1 A/B & $M R R 2^{\mathrm{WT}}:: \mathrm{FRT} / M R R 2^{\mathrm{WT}}:: \mathrm{FRT}$ & This study \\
\hline SCMRR2R2S2 A/B & $M R R 2^{\mathrm{S} 143 \mathrm{P}, \mathrm{L} 144 \mathrm{~V}, \mathrm{~T} 145 \mathrm{~A}, \mathrm{~S} 165 \mathrm{~N}, \mathrm{A459T}, \mathrm{S} 480 \mathrm{P}}:: \mathrm{FRT} / M R R 2^{\mathrm{S} 143 \mathrm{P}, \mathrm{L} 144 \mathrm{~V}, \mathrm{~T} 145 \mathrm{~A}, \mathrm{~S} 165 \mathrm{~N}, \mathbf{A 4 5 9 T}, \mathrm{S} 480 \mathrm{P}}:: \mathrm{FRT}$ & This study \\
\hline SCMRR2R3S3 A/B & $M R R 2^{183 \mathrm{~A}, \mathrm{~V} 451 \mathrm{~A}, \mathbf{V 5 8 2 L}}:: \mathrm{FRT} / M R R 2^{183 \mathrm{~A}, \mathrm{~V} 451 \mathrm{~A}, \mathbf{V 5 8 2 L}}:: \mathrm{FRT}$ & This study \\
\hline SCMRR2R4S4 A/B & $M R R 2^{\mathrm{R45Q}, \mathrm{V} 451 \mathrm{~A}}:: \mathrm{FRT} / M R R 2^{\mathrm{R45Q}, \text { V451A }}:: \mathrm{FRT}$ & This study \\
\hline SCMRR2R5S5 A/B & 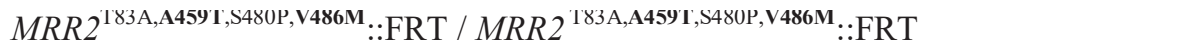 & This study \\
\hline SCMRR2R6S6 A/B & $M R R 2^{\underline{\mathrm{S} 466 \mathrm{~L}}, \underline{\mathrm{A} 468 \mathrm{G}}, \underline{\mathrm{S} 469 \mathrm{I}}, \underline{\mathrm{I} 470 \mathrm{~N}}, \mathrm{~S} 480 \mathrm{P}}:: \mathrm{FRT} / \mathrm{MRR} 2 \underline{\mathrm{S} 466 \mathrm{~L}, \underline{\mathrm{A} 468 \mathrm{G}}, \underline{\mathrm{S} 469 \mathrm{I}}, \underline{\mathrm{I} 470 \mathrm{~N}}, \mathrm{S480P}}:: \mathrm{FRT}$ & This study \\
\hline SCMRR2R8S8 A/B/C & $M R R 2^{C 9}::$ FRT / MRR2 $2^{C 9}::$ FRT & This study \\
\hline SCMRR2GADR1S1 A/B & MRR2-GAL4AD::FRT / MRR2-GAL4AD-3xHA::FRT & This study \\
\hline $\mathrm{SC} \Delta m r r 2 \mathrm{P}_{\mathrm{ADH} 1} \mathrm{MRR}^{\mathrm{WT}} \mathrm{A} / \mathrm{B}$ & $\Delta m r r 2:: \mathrm{FRT} / \Delta m r r 2:: \mathrm{FRT}, A D H 1 / a d h 1:: \mathrm{P}_{\mathrm{ADH} 1}-M R R 2^{\mathrm{WT}}$ & This study \\
\hline $\mathrm{SC} \Delta m r r 2 \mathrm{P}_{\mathrm{ADH} 1} \mathrm{MRR} 2{ }^{\mathrm{C} 9} \mathrm{~A} / \mathrm{B}$ & $\Delta m r r 2:: \mathrm{FRT} / \Delta m r r 2:: \mathrm{FRT}, A D H 1 / a d h 1:: \mathrm{P}_{\mathrm{ADH} 1}-M R R 2^{\mathrm{C} 9}$ & This study \\
\hline
\end{tabular}

${ }^{1}$ Tentative activating mutations in $M R R 2$ described in (118) are underlined.

${ }^{2} \mathrm{Amino}$ acid changes in $M R R 2$ in bold were discovered from this study. $M R R 2_{\mathrm{C} 9}$ refers to the $M R R 2$ allele of isolate $\mathrm{C} 9$ as described in (118) containing S143P, L144V, T145A, H358N, E439K, V451A, S466L, A468G, S469T, T470N, S480P mutations 
A

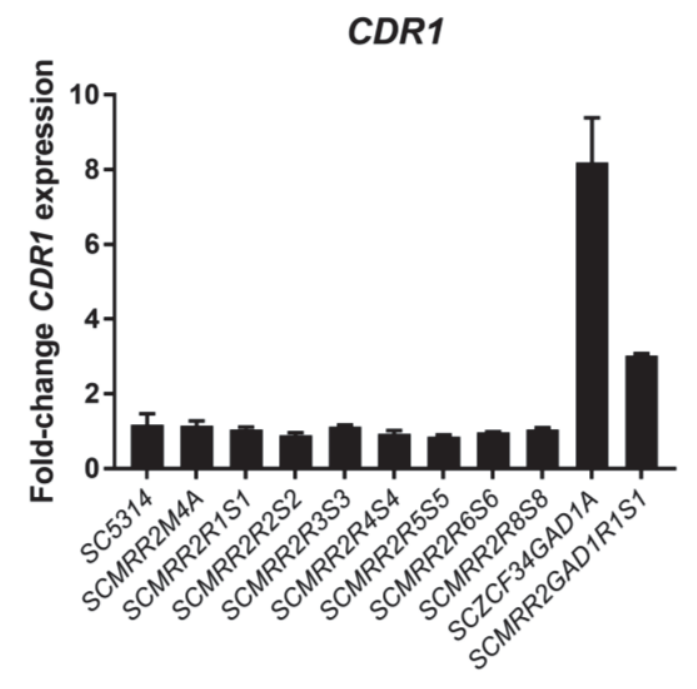

B

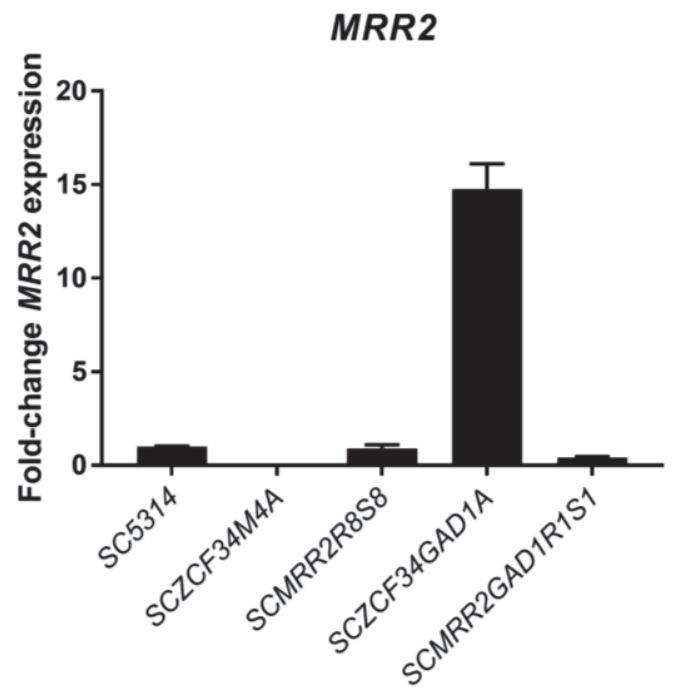

Figure 2-1. Fold-change in expression levels of $M R R 2$ mutant strains compared to SC5314

(A) Fold-change in expression levels compared to SC5314 of CDR1 for the $m r r 2 \Delta / \Delta$ strain (SCZCF34M4A), the mutant MRR2 strains containing MRR2 ${ }^{\mathrm{WT}}$ (SCMRR2R1S1), $M R R 2^{\mathrm{A} 459 \mathrm{~T}}$ (SCMRR2R2S2), MRR2 ${ }^{\mathrm{V} 582 \mathrm{~L}}$ (SCMRR2R3S3), MRR2 ${ }^{\mathrm{R} 45 \mathrm{Q}}$ (SCMRR2R4S4), $M R R 2^{A 459 T, \text { V486M }}$ (SCMRR2R5S5), MRR2 ${ }^{\mathrm{S} 466 \mathrm{~L}, \mathrm{A468G} \text {, S469T, T470N }}$ (SCMRR2R6S6),

$M R R 2^{\mathrm{C} 9}$ (SCMRR2R8S8) and artificially-activated Mrr2 strains expressed from either the $A D H 1$ promoter (SCZCF34GAD1A) or the native $M R R 2$ promoter (SCMRR2GAD1R1S1). (B) Fold-change in MRR2 expression levels compared to SC5314 for the $m r r 2 \Delta / \Delta$ strain (SCZCF34M4A), the mutant $M R R 2^{\mathrm{C} 9}$ strain (SCMRR2R8S8), the artificially-activated Mrr2 strains expressed from either the ADH1 promoter (SCZCF34GAD1A) or the native MRR2 promoter (SCMRR2GAD1R1S1). Expression was obtained in technical and biological triplicate for each strain tested and error bars reflect standard error of the mean. 


\section{Activated Mrr2 does not appear to regulate $M R R 2$ expression}

To determine if Mrr2 autoregulates its expression level, we measured MRR2 mRNA abundance of our created strains relative to SC5314 (Figure 2-1B). The mutant strain $M R R 2^{\mathrm{C} 9}$ and the artificially-activated $M R R 2$ strain $M R R 2-G A L 4 \mathrm{AD}-3 \mathrm{xHA}$ did not appear to have increased expression of $M R R 2$ compared to SC5314. As expected, strains with hyperactive $M R R 2$ expressed $M R R 2 \sim 14$-fold higher compared to SC5314.

Therefore, it appears only the relative overexpression of $M R R 2$ from the $A D H 1$ promoter and not activation of Mrr2 itself had any effect on increased MRR2 expression.

\section{Overexpression of wildtype or mutant $M R R 2$ in $C$. albicans strain does not affect fluconazole susceptibility or $C D R 1$ expression}

In order to investigate whether changes in $C D R 1$ expression and fluconazole susceptibility required strong constitutive expression of $M R R 2$, we constructed strains which lacked both native copies of $M R R 2$ but possessed a single copy of either the open reading frame of $M R R 2^{\mathrm{WT}}$ or $M R R 2^{\mathrm{C} 9}$ fused to the $A D H 1$ promoter. In both cases, $M R R 2$ mRNA expression was increased $>30$-fold compared to SC5314 in the independently created mutant strains mrr2 $/ \triangle$ adh1 $:: \mathrm{P}_{A D H 1}-M R R 2^{\mathrm{WT}} / A D H 1$ (SC $\Delta \mathrm{mrr}_{2} \mathrm{P}_{\mathrm{ADH} 1} \mathrm{MRR} 2^{\mathrm{WT}}$ $\mathrm{A} / \mathrm{B})$ and $m r r 2 \Delta / \triangle$ adh $1 \Delta:: \mathrm{P}_{A D H 1}-M R R 2^{\mathrm{C} 9} / A D H 1$ (SC $\Delta \mathrm{mrr} 2 \mathrm{P}_{\mathrm{ADH} 1} \mathrm{MRR} 2^{\mathrm{C} 9} \mathrm{~A} / \mathrm{B}$, Figure 2B). However, compared to SC5314, CDR1 expression was not markedly different for any of the strains, with no increase in constitutive CDR1 mRNA greater than 1.2-fold (Figure 2-2A). As expected, the strains also did not show any change in fluconazole susceptibility by either MIC or E-test (Table 2-2). Thus, overexpression of either $M R R 2^{\mathrm{WT}}$ or $M R R 2^{\mathrm{C} 9}$ does not appear to influence $C D R 1$ expression or fluconazole susceptibility in C. albicans.

\section{Discussion}

Gain-of-function mutations in the genes encoding fungus-specific ZCFs Tac1, Mrr1 and Upc2 have long been known to contribute to azole resistance in C. albicans. However, only recently was it reported that non-synonymous mutations found in the $M R R 2$ gene of clinical isolates impacted fluconazole susceptibility through increased Cdr1 efflux pump expression, indicating that $M R R 2$ may be a clinically relevant mechanism of azole resistance. Here, we sequenced a collection of predominantly fluconazole-resistant clinical isolates in order to uncover additional mutations in the $M R R 2$ gene that would contribute to fluconazole resistance. In total, mutations resulting in fifteen amino acid substitutions were identified across these clinical isolates. Three (R45Q, A459T, and V486M) were found uniquely in fluconazole-resistant clinical isolates and had not been previously reported. The remaining were described in a previous report or were present in fluconazole-susceptible isolates and therefore not likely directly involved in azole resistance. Our results indicate that none of the tested SNPs in $M R R 2$ have an impact on susceptibility to fluconazole. Similarly, CDR1 expression also did not appear to be affected by the presence of any of the tested SNPs. 
A

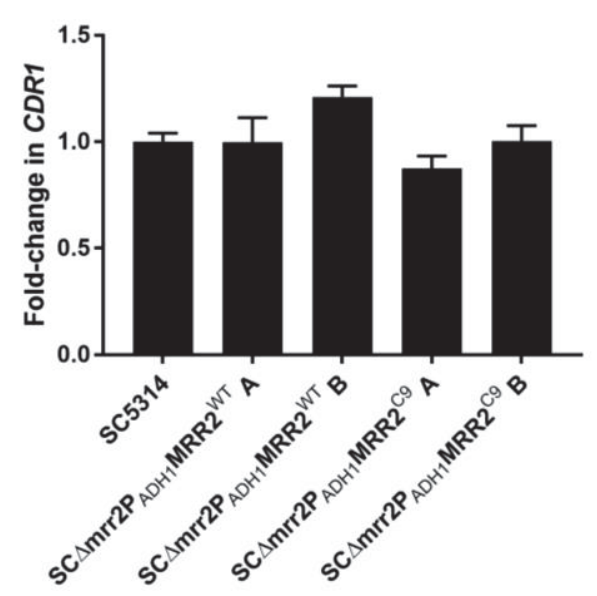

B

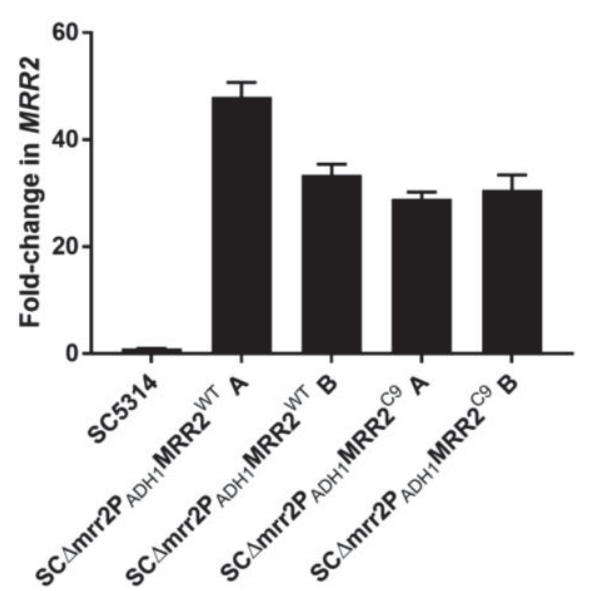

Figure 2-2. Fold-change in $C D R 1$ and $M R R 2$ expression compared to SC5314

(A) Fold-change in expression levels compared to SC5314 of CDR1 for strains possessing either $M R R 2^{\mathrm{WT}}$ (SC $\triangle \mathrm{mrr} 2 \mathrm{P}_{\mathrm{ADH} 1} \mathrm{MRR} 2^{\mathrm{WT}} \mathrm{A}$ and $\mathrm{B}$ ) or mutant $M R R 2^{\mathrm{C} 9}$

(SC $\triangle \mathrm{mrr}_{2} \mathrm{P}_{\mathrm{ADH}} \mathrm{MRR} 2^{\mathrm{C} 9} \mathrm{~A}$ and $\mathrm{B}$ ) expressed via the $A D H 1$ promoter. (B) Fold-change in MRR2 expression levels compared to SC5314 for the same strains shown in (A).

Expression was obtained in technical and biological triplicate for each strain tested and error bars reflect standard error of the mean. 
In particular, four mutations identified in isolates in our collection ( $\mathrm{S} 466 \mathrm{~L}$, A468G, S469T, and T470N) had been previously shown to impact fluconazole susceptibility. The amino acid substitutions S466L and T470N had also been reported to increase $C D R 1$ expression by at least two-fold as compared to a control strain. In our hands the amino acid substitutions S466L, A468G, S469T, and T470N do not appear to have any effect on susceptibility to fluconazole and $C D R 1$ expression does not increase in the mutant strains containing these Mrr2 amino acid substitutions compared to SC5314. This finding is consistent with our clinical isolates that possess these changes in Mrr2 but do not highly express $C D R 1$.

Since the publication of the original report first describing them, mutations in $M R R 2$ have been referenced as a clinically relevant mechanism of fluconazole resistance $(160,197,198)$. While our results confirm that artificial activation of the Mrr2 ZCF indeed results in $C D R 1$ upregulation and a consequent increase in fluconazole resistance as previously described (117), we were unable to replicate the changes attributed to these mutations in a previous report (118). We were unsuccessful in obtaining isolate $\mathrm{C} 9$ from this previous report, which contained the MRR2 mutations in question, and as such could not make a direct assessment of this clinical isolate. One possibility for the disparity seen between our results and those reported previously could be differences in methodology. We expressed MRR2 from its native promoter, including replacement of both ORFs in the $M R R 2$ locus, in order to mimic natural Mrr2 expression. This is in contrast to the methods used previously whereby $M R R 2$ was placed in the $A D E 2$ locus utilizing a single copy of the ORF under control of the $A D H 1$ promoter. This could possibly cause altered metabolic burdens in these strains compared to ours. Furthermore, the $A D H 1$ promoter has been described to have variability in activity over time under different conditions (199), and therefore Mrr2 protein levels may be different depending on the time at which cells were harvested or observed for assays. Lastly, we used 50\% inhibition from the fluconazole-free control well for identifying the MIC of our strains, as directed by the CLSI reference method for broth microdilution antifungal susceptibility testing of yeast $(200,201)$. The MICs presented in the previous report were identified by $80 \%$ inhibition of cell growth in the control well, which could explain increased MICs observed in $M R R 2$ mutant strains, especially if the trailing growth phenomenon was observed in these strains.

Given the lower expression of $M R R 2$ that we observed in our strains using the native $M R R 2$ promoter compared to hyperactive Mrr2, which overexpressed $M R R 2$ via fusion to the $A D H 1$ promoter, we considered that $M R R 2$ was not being sufficiently expressed in our strains to allow for detection of changes in CDR1 expression or fluconazole susceptibility. However, the MRR2-GAL4AD-3xHA strain expressing artificially-activated Mrr2 via its native promoter demonstrates that native $M R R 2$ expression levels are sufficient to observe changes in fluconazole MIC and CDRI expression. Moreover, we believe our strains, which tested the effects of the homozygous SNPs in MRR2 at the native locus, more closely approximates the effects these nucleotide changes would have in a clinical isolate. Lastly, we constructed our own $\mathrm{P}_{A D H 1}-M R R 2$ overexpressing strains to test whether there were differences in $C D R 1$ expression or fluconazole susceptibility between strong, constitutive expression of either 
$M R R 2^{\mathrm{WT}}$ or $M R R 2^{\mathrm{C} 9}$ and were unable to discern any phenotype differences between the strains. Overall, in all strains and conditions tested here we were unable to detect any change in $C D R 1$ expression or fluconazole susceptibility between strains containing mutant $M R R 2$ versus wildtype.

The ATP-binding cassette transporter Cdr1 has been shown to be regulated not only by Tac1, but also Ndt80. Furthermore, it has been shown that through artificial activation and overexpression, the zinc cluster transcription factors Mrr2, Znc1, and Stb5 also can influence Cdr1 expression levels. Thus, we speculate that there remain additional determinants of efflux pump upregulation that have clinical relevance. However, our experiments indicate that the nucleotide changes observed here, and reported previously, in MRR2 do not impact fluconazole susceptibility or CDR 1 expression and are not clinically relevant to fluconazole resistance in $C$. albicans. Given that azole resistance cannot be fully explained by known mechanisms, further investigation of other ZCFs in azole resistance is warranted.

\section{Materials and Methods}

\section{Strains and growth conditions}

Table 2-2 lists the C. albicans strains used in this study. C. albicans isolates were obtained from a repository of clinical isolates at the University of Iowa and have been previous reported elsewhere (115). Strains were stored in $40 \%$ glycerol frozen stocks at $80^{\circ} \mathrm{C}$. Routine growth of cells was performed in YPD liquid media ( $1 \%$ yeast extract, $2 \%$ peptone, $2 \%$ dextrose $)$ at $30^{\circ} \mathrm{C}$. Nourseothricin-containing $(200 \mu \mathrm{g} / \mathrm{mL})$ YPD agar plates were used for selection of strains containing the $S A T 1$ marker. For plasmid propagation, $\mathrm{DH} 5 \alpha^{\mathrm{TM}}$ competent $E$. coli cells (Invitrogen) were grown in Luria-Bertani (LB) broth or on LB agar plates containing either $100 \mu \mathrm{g} / \mathrm{mL}$ ampicillin or $50 \mu \mathrm{g} / \mathrm{mL}$ kanamycin.

\section{Plasmid construction}

Plasmids were derived from plasmid strain pBSS2, which contains the SAT1 flipper disruption cassette from pSFS2 (202) placed in the pBluescript II KS+ vector. The 3' flanking region of the MRR2 ORF was amplified from SC5314 genomic DNA using primers CaMRR2C_(NotI) and CaMRR2D_(SacI) and ligated into pBSS2 at the NotI and SacI restriction sites to create pMRR2CD. The 5' upstream region and $M R R 2$ ORF of isolates SC5314, 20, 22, 23, 28, and 29 were amplified using primers CaMRR2A (KpnI) and CaMRR2E (XhoI) and cloned into pMRR2CD to obtain plasmids pBSS2-MRR2 ${ }^{\mathrm{WT}}$, pBSS2-MRR2 ${ }^{20}, \mathrm{pBSS}^{2-M R R 2}{ }^{22}, \mathrm{pBSS}^{2-M R R} 2^{23}, \mathrm{pBSS} 2-$ $\mathrm{MRR}^{28}$, and pBSS2-MRR2 ${ }^{29}$, respectively. For pMRR2_C9.3 which replicated the $M R R 2$ allele of isolate C9 described by Wang et al. (118), primers CaMRR2_P1_F and CaMRR2_C9_R were used to amplify a 5' portion of the MRR2 ORF from genomic 
DNA of isolate 33. The resulting amplicon was fused to a synthesized gBlocks fragment (Integrated DNA Technologies) containing the 3' portion of the MRR2 ORF using CaMRR2_Nested_F_KpnI and MRR2_Nested_DR_XhoI and cloned into pMRR2CD to give pMRR2_C9.2. Final changes of nucleotides were accomplished using short, overlapping extension (SOE) PCR on plasmid pMRR2_C9.2 using primer pairs pBSS2_1F.2 and CaMRR2_C9.2_R6, CaMRR2_C9.2_F6 and CaMRR2_C9.2_R7, CaMRR2_C9.2_F7 and CaMRR2_C9.2_R8, and CaMRR2_C9.2_F8 and pBSS2_ MAL2_R to generate amplicon fragments of the MRR2 ORF. Fragments were fused together via SOE PCR using CaMRR2_Nested_F_KpnI and CaMRR2_Nested_ DR_XhoI and were cloned into pMRR2CD to create pMRR2_C9.3. Plasmid pMRR2GAD1 was generated using genomic DNA from SCZCF34GAD1A and primers CaMRR2GAD1A_F KpnI and 3XHA_ACT1_R XhoI to amplify the MRR2 ORF including the fused $\bar{G} A L 4$ activation domain and $\overline{3}$ X HA-tagged C-terminal region. The resulting amplicon was cloned into pMRR2CD to yield plasmid pMRR2GAD1. Plasmids $\mathrm{pP}_{\mathrm{ADH} 1}-\mathrm{MRR} 2^{\mathrm{WT}}$ and $\mathrm{pP}_{\mathrm{ADH} 1}-\mathrm{MRR} 2^{\mathrm{C} 9}$ were used to express the open reading frame of $M R R 2$ from the $A D H 1$ locus, utilizing the $A D H 1$ promoter and termination sequences. The MRR2 open reading frame of either SC5314 or the C9 isolate was fused to the 3' $A D H 1$ terminator via overlap extension PCR utilizing primers CaMRR2_2F EcoRI, CaMRR2_R_ADH1t.2, CaADH1t_F, and ADH1t_R_XhoI. The $A D H 1$ promoter was PCR amplified using primers CaPADH1_AF_KpnI and CaPADH1_2R_EcoI. Both the $A D H 1$ promoter and fused $M R R 2-A D H 1 \mathrm{t}$ amplicons were digested with either KpnI and EcoRI or EcoRI and XhoI, respectively, and subsequently ligated into the KpnI-XhoI linearized plasmid pADH1CD, a pBSS2 derivative containing the 342 bp 3' flanking homology near the $A D H 1$ locus generated with primers CaADH1_C_SacII and CaADH1_D_NcoI_SacI. Successful transformants were screened on LB agar plates containing $50 \mu \mathrm{g} / \mathrm{mL}$ ampicillin and sequenced for the MRR2 open reading frame corresponding to either SC5314 or isolate $\mathrm{C} 9$ for plasmid $\mathrm{pP}_{\mathrm{ADH}} 1^{-\mathrm{MRR} 2} 2^{\mathrm{WT}}$ and $\mathrm{pP}_{\mathrm{ADH}}{ }^{-}$ MRR2 ${ }^{\mathrm{C} 9}$, respectively.

\section{$M R R 2$ amplification and sequencing}

Table 2-4 lists the primers used for $M R R 2$ amplification and sequence verification. The $M R R 2$ coding sequence of each isolate was PCR amplified from genomic DNA using primers CaMRR2_F_Amp and CaMRR2_R_Amp. PCR products were purified using the QIAquick ${ }^{\circledR}$ PCR Purification Kit (Qiagen) and product was sequenced on an $\mathrm{ABI} 3130 \mathrm{XL}$ genetic analyzer using $M R R 2$ sequencing primers. Sequencing was accomplished in duplicate in independently grown isolates.

\section{Candida albicans strain construction}

The $S A T 1 /$ FLP-containing MRR2 replacement cassette was excised from plasmids

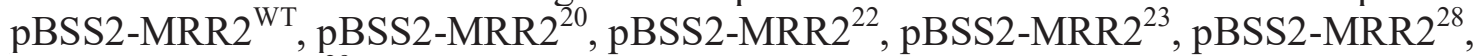
and pBSS2-MRR2 ${ }^{29}$, pMRR2_C9.3, and pMRR2GAD1 were digested using KpnI-HF and NcoI-HF restriction enzymes and transformed via electroporation into C. albicans 


\section{Table 2-4. Primers used in this study}

\begin{tabular}{|c|c|}
\hline Primer name by purpose & Sequence $^{1}$ \\
\hline \multicolumn{2}{|l|}{ Amplification } \\
\hline CaMRR2 F Amp & 5' - TACGAAATACTTGGAGTTATTCCCTAC - 3' \\
\hline CaMRR2_R_Amp & 5' - CTAGTTTTGTGTCTAGTTCTATTGTTATTG - 3’ \\
\hline CaMRR2GĀD1A_F_KpnI & $\begin{array}{l}\text { 5' - CAGGGTACCAACTTGAAAAATTGCTCAACTCTTATATAGCAAAAATAAACAACCAAT } \\
\text { AGCTTCTTCGCCAATGACCAAACGTGATCGTAC - 3, }\end{array}$ \\
\hline 3XHA_ACT1_R_XhoI & 5' - CTACTCGAGGATTTCCAGAATTTCACTCTTA - 3' \\
\hline CaPADH1_AF KpnI & 5' - GATGGTACCACTACCACTGCAGCTGCATC - 3' \\
\hline CaMRR2_2R_EcoRI & 5' - CTTTTTGAGTTTTTGGGATTTGTTCGAATTCAATTGTTTTTGTATTTGTTGTTGTTGTTG - 3' \\
\hline CaMRR2_2F_EcoRI & 5' - GATGAATTCATGACCAAACGTGATCGTACAAT - 3' \\
\hline CaADH1t_R_X̄XhoI & 5' - CATCTCGAGTTAACCAAAATCAACGACAAATTG - 3' \\
\hline CaADH1_C_SacII & 5' - GATCCGCGGCATTGATTGTTTGTGTTAGTTTTTCA - 3' \\
\hline CaADH1_D_NcoI_SacI & 5' - GATGAGCTCCCATGGAACACCCAGTTTAATTTCCATGA - 3' \\
\hline \multicolumn{2}{|l|}{ Sequencing } \\
\hline CaMRR2SeqA & 5' - GCAGAAGCGAGGGAACTTGAAA - 3' \\
\hline CaMrR2SeqB & 5' - ACTTGGAGAAGCATACATACCGAG - 3' \\
\hline CaMRR2SeqC & 5' - TACTCGCTCGCCTTACATCGA - 3' \\
\hline CaMRR2SeqD & 5' - АATCTCAАCTACATCCACCTTGTC - 3' \\
\hline CaMRR2SeqE & 5' - CGAAACTTCTGCCATCCTCAAT - 3' \\
\hline CaMRR2SeqF & 5' - GTACATCGGACGACCGTTCC - 3' \\
\hline CaMRR2SeqG & 5' - CTATACTTTGCTCCATTGGCGG - 3' \\
\hline CaMRR2SeqH & 5' - GAACGATGTTAATGGGTCAGCAAAG - 3' \\
\hline \multicolumn{2}{|l|}{ Short, overlapping extension } \\
\hline CaMRR2_P1_F & 5' - CACTGTGATCGGTTATCTTTGTTGCAC - 3' \\
\hline CaMRR2_C9 3R & 5’ - GTTGCTTGGGGTTGTTTTCGCCAATG - 3’ \\
\hline CaMRR2_Nested_F_KpnI & 5' - AGCGGTACCTTGGACTTTGACTGTTCAGA - 3' \\
\hline $\begin{array}{l}\text { CaMRR2_Nested_DR_XhoI } \\
\text { pBSS2 } 1 \text { F. } 2\end{array}$ & 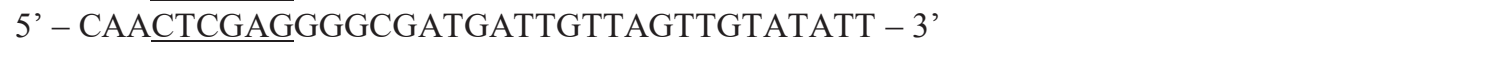 \\
\hline pBSS2_MAL2_R & 5' - CGTGGTTTCAGTGGCTACAAC - 3' \\
\hline CaMRR2_C9.2_F6 & 5' - GGGCCCACAGATCGACGGACATTAC - 3' \\
\hline CaMRR2_C9.2_R6 & 5' - GTAATGTCCGTCGATCTGTGGGCCC - 3' \\
\hline CaMRR2_C9.2_F7 & 5' - CATTAACATCGTTCTTTAGTCTCATCCC - 3' \\
\hline CaMRR2_C9.2_R7 & 5' - GGGATGAGACTAAAGAACGATGTTAATG - 3' \\
\hline
\end{tabular}




\section{Table 2-4. (Continued)}

\begin{tabular}{|c|c|}
\hline Primer name by purpose & Sequence $^{1}$ \\
\hline CaMRR2_C9.2_F8 & 5' - CAGATTTACCAGTTGCCAAAAGAC - 3' \\
\hline CaMRR2_C9.2_R8 & 5' - GTCTTTTGGCAACTGGTAAATCTG - 3' \\
\hline CaMRR2_2F_EcoRI & 5' - GATGAATTCATGACCAAACGTGATCGTACAAT - 3' \\
\hline CaMRR2_R_ADH1t.2 & 5' - GCTATTTGCTTACGAGATTTTGAGGAAATCCC - 3' \\
\hline CaADH1t_F- & 5' - CAAAATCTCGTAAGCAAATAGCTAAATTATATACG - 3' \\
\hline CaADH1t_R_XhoI & 5' - CATCTCGAGTTAACCAAAATCAACGACAAATTG - 3' \\
\hline \multicolumn{2}{|l|}{ Real-time qPCR } \\
\hline CaMRR2_qPCR_F & 5' - TCCAAGTAAGTGTGGGTGTCC - 3' \\
\hline CaMRR2_qPCR_R & 5' - ATGTAAGGCGAGCGAGTAGC - 3' \\
\hline CaCDR1-f_qPCR & 5' - ATTCTAAGATGTCGTCGCAAGATG - 3' \\
\hline CaCDR1-R_qPCR & 5' - AGTTCTGGCTAAATTCTGAATGTTTTC \\
\hline CaACT1-FW̄'_qPCR & 5' - ACGGTGAAGAAGTTGCTGCTTTAGTT - 3' \\
\hline CaACT1-rvs_qPCR & 5' - CGTCGTCACCGGCAAAA - 3' \\
\hline
\end{tabular}

${ }^{1}$ Underlined nucleotide indicates introduction of a restriction site sequence. 
strain SCZCF34M4A to generate strains heterozygous for their respective MRR2 alleles of interest. Figure 2-3 diagrams the general strain construction method for allelic replacement of MRR2 at the native locus (Figure 2-3A) and inserted at the ADH1 locus (Figure 2-3B). The nourseothricin marker in all strains was recycled by FLP recombinase induction after 48 hours growth in YPD liquid media. Repeat transformation of the resultant strains generated the homozygous $M R R 2$ allele replacements MRR2 ${ }^{\mathrm{WT}}$ (SCMRR2R1S1), MRR2 ${ }^{\text {S143P, L144V, T145A, S165N, A459T, S480P }}$ (SCMRR2R2S2), MRR2 $2^{\text {T83A, V451A, V582L }}$ (SCMRR2R3S3), MRR2 $2^{\mathrm{R} 45 \mathrm{Q}, \mathrm{V} 451 \mathrm{~A}}$ (SCMRR2R4S4), MRR2 ${ }^{\text {T83A, A459T, S480P, V486M }}$ (SCMRR2R5S5), MRR2 ${ }^{\mathrm{S} 466 \mathrm{~L}, \mathrm{~A} 468 \mathrm{G}, \mathrm{S} 469 \mathrm{~T} \text {, }}$ T470N, S480P (SCMRR2R6S6), MRR2 ${ }^{\mathrm{C} 9}$ (SCMRR2R8S8), and MRR2-GAL4AD-3xHA (SCMRR2GAD1R1S1), respectively. MRR2 allelic replacements were confirmed by Southern blot and confirmation of polymorphisms or fused domains present in generated strains were accomplished through Sanger sequencing. For strains $\mathrm{SC} \Delta m r r 2 \mathrm{P}_{\mathrm{ADH} 1} \mathrm{MRR} 2^{\mathrm{WT}}$ and $\mathrm{SC} \Delta m r r 2 \mathrm{P}_{\mathrm{ADH} 1} \mathrm{MRR} 2^{\mathrm{C} 9}$, plasmids $\mathrm{pP}_{\mathrm{ADH} 1}-\mathrm{MRR} 2^{\mathrm{WT}}$ and $\mathrm{pP}_{\mathrm{ADH} 1}-\mathrm{MRR} 2{ }^{\mathrm{C} 9}$ were similarly digested with KpnI-HF and NcoI-HF to excise and purify the $M R R 2$-containing $S A T 1 / \mathrm{FLP}$ cassette targeting the $A D H 1$ locus. Paired doubled strand breaks near the 5' and 3 ' ends of the $A D H 1$ were induced utilizing the CRISPRCas9 technology using previously described methods (203). Briefly, 100 pmol Alt-R® CRISPR-Cas9 tracrRNA (Integrated DNA Technologies, Inc.) was duplexed with an equal amount of crRNA targeting either the $5^{\prime}$ or $3^{\prime}$ region of the $A D H 1$ locus at $95^{\circ} \mathrm{C}$ for 5 minutes. The duplexed guide RNA was then complexed with 2 ug of Alt-R® S.p. HiFi Cas9 Nuclease V3 (Integrated DNA Technologies, Inc.) to form the resulting ribonucleoprotein complex. Approximately $1 \mathrm{ug}$ of purified digests from either $\mathrm{pP}_{\mathrm{ADH} 1^{-}}$ $\mathrm{MRR}^{\mathrm{WT}}$ or $\mathrm{pP}_{\mathrm{ADH1}}-\mathrm{MRR} 2{ }^{\mathrm{C} 9}$ were transformed via electroporation along with Cas9 ribonucleoprotein complex into SCZCF34M4A to create SC $\triangle m r r 2 \mathrm{P}_{\mathrm{ADH} 1} \mathrm{MRR}{ }^{\mathrm{WT}}$ and $\mathrm{SC} \Delta m r r 2 \mathrm{P}_{\mathrm{ADH} 1} \mathrm{MRR} 2{ }^{\mathrm{C} 9}$ expressing a single copy of the $M R R 2$ open reading frame from the $A D H 1$ locus.

\section{Azole susceptibility testing}

Fluconazole MICs were determined using the broth microdilution methods as described by the Clinical Laboratory and Standards Institute (200, 201), with slight modification by addition of 2\% glucose to Roswell Park Memorial Institute 1640 (RPMI) media to reduce trailing growth in wells. Fluconazole concentration for MICs ranged from 256 to $0.06 \mu \mathrm{g} / \mathrm{mL}$. Measurements were read visually at 24 and 48 hours after incubation at $35^{\circ} \mathrm{C}$ for a $50 \%$ reduction in growth from drug-free control wells. For fluconazole susceptibility testing using E-test strips (bioMérieux), cells were diluted to an $\mathrm{OD}_{600}$ of 0.100 and swabbed using sterile cotton tips onto RPMI-agar plates. MICs were visually read at the border of the zone of inhibition after 24 and 48 hours of incubation at $35^{\circ} \mathrm{C}$. 
Figure 2-3. Representative schematic of $M R R 2$ mutant strain construction in $C$. albicans

The diagram shows the MRR2/CaSAT1/FLP cassette integration at either (A) the native $M R R 2$ gene locus or (B) replacing the $A D H 1$ open reading frame at the $A D H 1$ locus. The black line represents the sequence of a single allele of genomic DNA. Open reading frames are depicted as filled arrows along the genomic sequence with gene names labeled. Promoter regions, terminators, and the FLP recognition target sequences are represented by bent arrows, hairpins, and black triangles, respectively. The double slashes interrupting the genomic DNA sequence in (B) represent the CRISPR-Cas9 cutting sites. The homologous 5' and 3' flanking sequences for targeted fragment integration are represented as forward-striped and backwards-striped rectangles, respectively. 
A
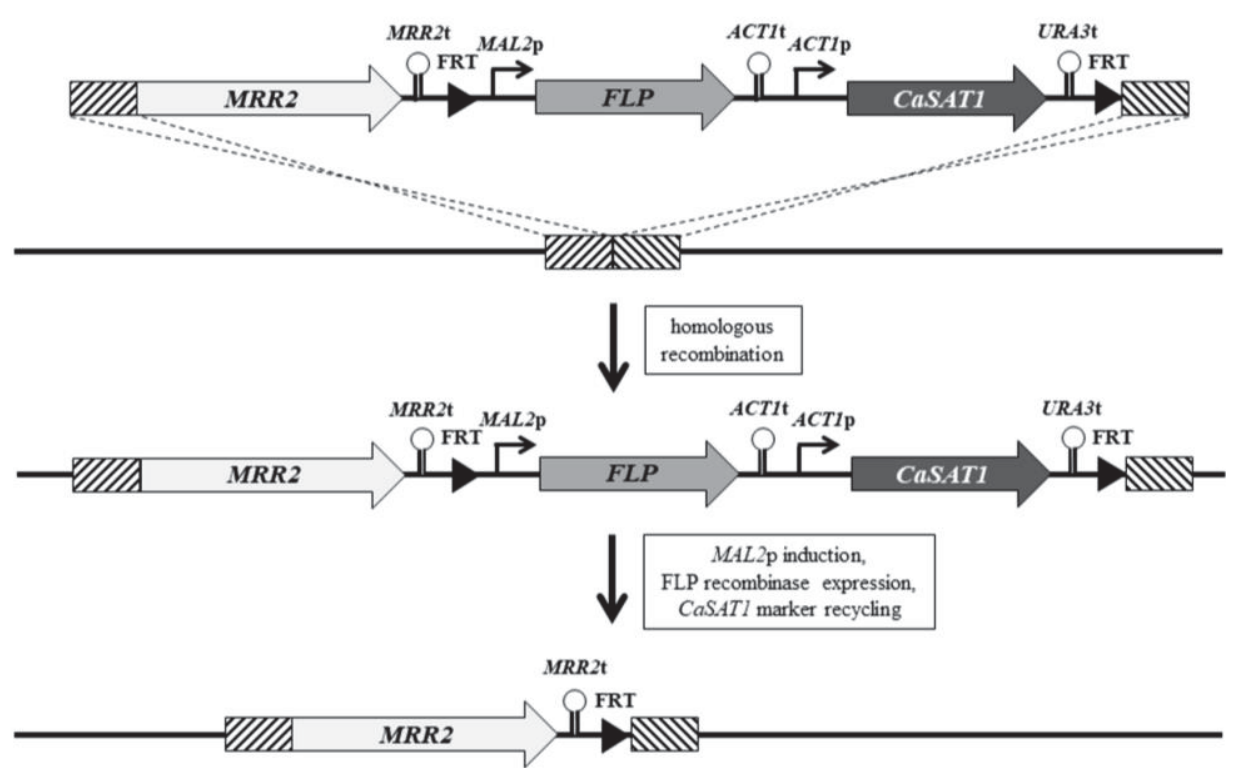

B
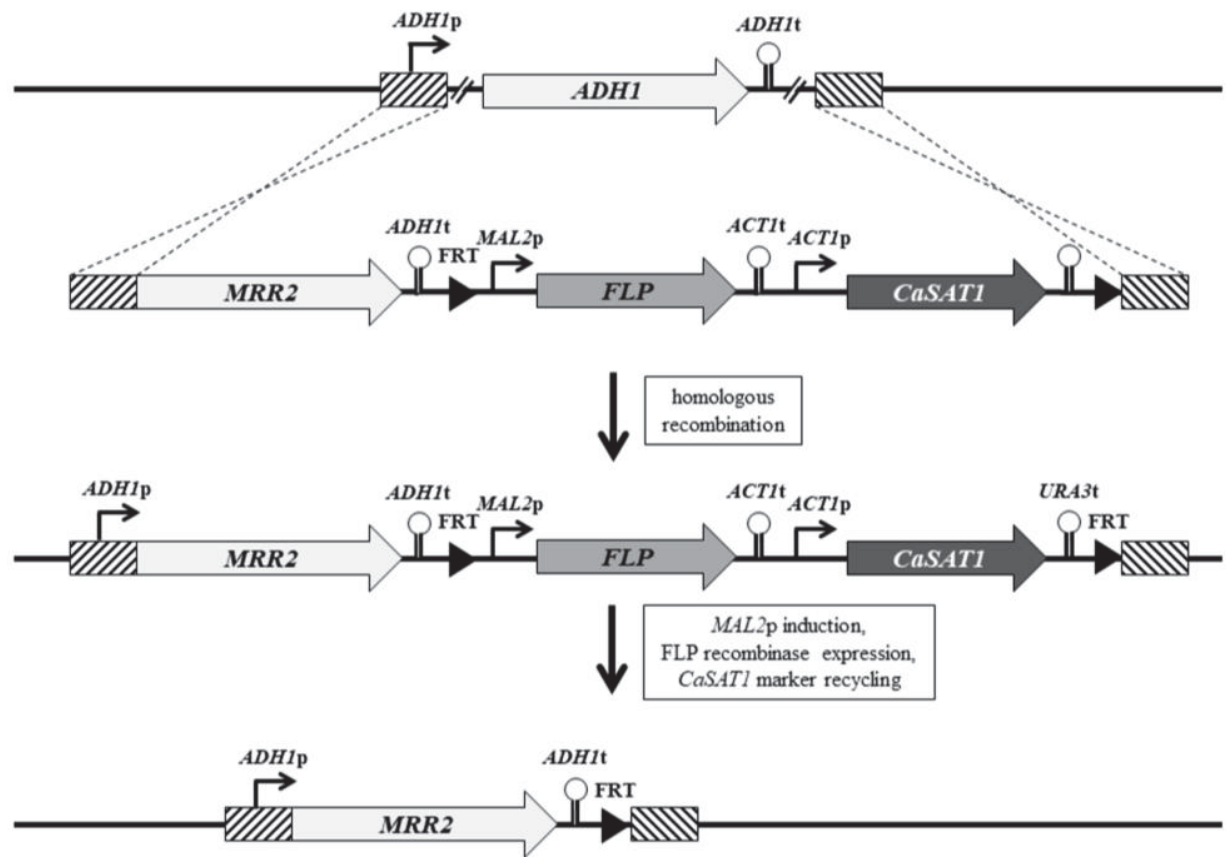


\section{Quantitative real-time reverse transcription PCR}

First strand cDNAs were synthesized from $1 \mu \mathrm{g}$ of total RNA using the SuperScript ${ }^{\circledR}$ VILO $^{\text {TM }}$ for qRT-PCR (Invitrogen). Real-time PCR primers for ACT1, $C D R 1$ and MRR2 were synthesized by Integrated DNA Technologies (Table 2-4). Relative mRNA abundance of $C D R 1$ and $M R R 2$ was measured in triplicate on the StepOnePlus Instrument (Applied Biosystems) against the endogenous control gene $A C T 1$ and the reference strain SC5314. To assess statistical significance, standard error of the mean of the average expression levels of each isolate measured in triplicate was calculated.

\section{Nucleotide sequence accession numbers}

The coding sequences of the MRR2 alleles described in this study have been

deposited in GenBank under the accession numbers [MK332630] through [MK332702]. 


\section{CHAPTER 3. MUTATIONS IN ERG11 HAVE VARYING CONTRIBUTIONS TO AZOLE RESISTANCE, ERG11 PROTEIN FUNCTION, AND COMPETITIVE GROWTH FITNESS OF THE CELL ${ }^{2}$}

\section{Introduction}

Candida albicans is a polymorphic, opportunistic pathogen responsible for causing a wide range of infections. In the United States, C. albicans is the most common cause of invasive candidiasis $(59,190)$. Morbidity and mortality rates for invasive candida infections such as candidemia remain high. Such infections have a significant impact on the health care costs. In 2014, it was estimated that Candida infections in the US accounted for more than 25,000 hospitalizations totaling $\$ 1.4$ billion, with invasive candidiasis contributing to $84 \%$ of the incurred costs (68). Even the less serious superficial Candida infections such as vulvovaginal or oropharyngeal candidiasis were responsible for over 3.5 million outpatient visits that year at an expense of nearly $\$ 2.1$ billion.

In cases of both invasive and non-invasive Candida infection, the azole antifungals have been and continue to be an important agent used in treatment. The azoles work through inhibition of the ERG11-encoded protein $14 \alpha$-lanosterol demethylase, also known as the CYP51 enzyme. While resistance in C. albicans to azoles like fluconazole has remained relatively constant in recent years, azole-resistant Candida continues to be a serious threat needing to be addressed $(34,184,204)$.

Mechanisms of azole resistance often involve the regulation and overexpression of genes controlled by the zinc-cluster transcription factors (ZCFs), a fungal-specific transcription factor family. The ZCFs Tac1 and Mrr1 have been well-studied in their regulation of the expression of the ATP-binding cassette transporters Cdr1 and Cdr2 and the major facilitator superfamily transporter Mdr1, respectively $(82,83,104,105)$. The ZCF Upc2 is responsible for the regulation of most ergosterol biosynthesis genes, including ERG11, which encodes for the azole drug target (Erg11/14 $\alpha$-lanosterol demethylase, hereafter referred to as CaCYP51), and can also influence azole susceptibility through overexpression of ERG11 (108, 110). Mutations in ERG11 resulting in amino acid substitutions that directly alter the drug target $14 \alpha$-lanosterol demethylase's protein sequence are another common azole resistance mechanism (121, $122,131)$. It is commonly a combination of these mechanisms that confer azole resistance in clinical isolates of C. albicans (107).

\footnotetext{
${ }^{2}$ Portions of chapter from previously published article; final submission modified with open access permission. Warrilow AG, Nishimoto AT, Parker JE, Price CL, Flowers SA, Kelly DE, Rogers PD, Kelly SL. 2019. The Evolution of Azole Resistance in Candida albicans Sterol 14alpha-Demethylase (CYP51) through Incremental Amino Acid Substitutions. Antimicrob Agents Chemother. doi:10.1128/AAC.0258618.
} 
To date, over 160 CaCYP51 amino acid substitutions have been reported in clinical isolates of $C$. albicans $(131,134,136,139)$. While mutation hot spots have been noted where amino acid substitutions cluster, the breadth of substitutions in CaCYP51 covers virtually the entire length of the 528 amino acid protein (Figure 3-1) (205). However, the effects of these substitutions are variable - while some have been shown to confer resistance to fluconazole and other azoles, others have never been shown to affect susceptibility to the azoles. A previous report by Flowers et al. showed the individual contributions of several amino acid substitutions in CaCYP51 and also found that several double substitutions conferred greater azole resistance than any single substitution, indicating that the presence of multiple CaCYP51 mutations may be beneficial for the cell in the presence of azoles (139).

More recently, the crystal structures of CaCYP51 complexed with azole antifungals have been analyzed and residues of significance in the catalytic domain or in contact with the drug ligands have been identified $(140,206)$. For example, while some residues like Tyr-132 lie exposed on the active site cavity of CaCYP51 and predictably would interfere with fluconazole binding, other residues such as the Ser-405 do not appear to contact the azoles, yet amino acid substitutions at this residue still confer azole resistance. Differences in the relative locations on CaCYP51 present an interesting question as to how amino acid substitutions might have variable effects on CYP51 enzyme function.

There have been significant recent findings regarding the differences in catalytic activity between amino acid substitutions in CaCYP51 activity, biochemical tolerance to the azoles, and azole binding affinity (141). In an effort to characterize functional and biochemical differences between enzymes containing substitutions in CYP51, purified recombinant CaCYP51 modified for expression in E. coli and 4x histidine-tagged for purification was synthesized for biochemical analysis. Twenty-four single amino acid substitution CaCYP51 mutants and five double amino acid substitution mutants were synthesized and purified, representing documented mutations found in clinical isolates. A wild type enzyme lacking any mutations representing the native CaCYP51 enzyme found in azole-susceptible clinical isolates was also synthesized for comparison. The catalytic turnover rate of the functioning enzyme was determined by quantification of sterol product form after addition of the CaCYP51 substrate lanosterol and the human cytochrome P450 reductase to the CaCYP51 enzyme. Even in the absence of azoles, CaCYP51 mutants varied widely in their catalytic turnover compared to the wild type. For example, while the V456I and Q474K substitutions seemingly increased relative velocity of the enzyme over the wild type by more than $50 \%$ at baseline, the Y118A, G307S, Y132H\&K143R, and G307S\&G450E substitutions all resulted in catalytic turnover of less than 10\% that of the wild type enzyme's turnover (Table 3-1).

In the presence of either $4 \mu \mathrm{M}$ fluconazole, voriconazole, itraconazole, and posaconazole, the wild type CaCYP51 enzyme retained less than $1 \%$ of its residual activity (Table 3-2). Many CaCYP51 mutants behaved in a similar fashion in the presence of the azoles, suggesting that they presumably would not influence azole 
1 MAIVETVIDG INYFLSLSVT QQISILLGVP FVYNLVWQYL YSLRKDRAPL

51 VFYWIIPWFGS AASYGQQPYE FFESCRQKYG DVFSFMLLGK IMTVYLGPKG

101 HEFVFNAKLS DVSAEDAYKH LTTPVFGKGV IYDCPNSRLM EQKKFAKFAL

151 TTDSFKRYVP KIREEILNYF VTDESFKLKE KTHGVANVMK TQPEITIFTA

201 SRSLFGDEMR RIFDRSFAQL YSDLDKGFTP INFVFPNLPL PHYWRDAAQ

251 KKISATYMKE IKSRRERGDI DPNRDLIDSL LIHSTYKDGV KMTDQEIANL

301 LIGILMGGQH TSASTSAWFL LHLGEKPHLQ DVIYQEVVEL LKEKGGDLND

351 LTYEDLQKLP SVNNTIKETL RMHMPLHSIF RKVTNPLRIP ETNYIVPKGH

401 YVLVSPGYAH TSERYFDNPE DFDPTRWDTA AAKANSVSFN SSDEVDYGFG

451 KVSKGVSSPY LPFGGGRHRC IGEQFAYVQL GTILTTFVYN LRWTIDGYKV

501 PDPDYSSMVV LPTEPAEIIW EKRETCMF*

Figure 3-1. Reported locations of CaCYP51 amino acid substitutions in C. albicans

Amino acid sequence of the 528 amino acid length of the CaCYP51 protein. Highlighted amino acids represent documented substitution(s). Yellow, green, blue, and purple highlights represent one, two, three and four possible different amino acid substitutions discovered, respectively. Bold lettering represents a difference in the SC5314 CaCYP51 reference sequence from the reported source's original amino acid residue at that location. 
Table 3-1. Yield and relative velocities of CaCYP51 proteins

\begin{tabular}{lcc}
\hline CaCYP51 substitution & Yield $^{\mathbf{1}}\left(\mathbf{n m o l ~ ~ ^ { - 1 }}\right)$ & Relative velocity $^{\mathbf{2}}$ \\
\hline Wild type & 119 & $1.000 \pm 0.047$ \\
Y118A & 49 & $0.029 \pm 0.001$ \\
F126V & 235 & $0.298 \pm 0.008$ \\
Y132F & 53 & $0.795 \pm 0.005$ \\
Y132H & 28 & $0.135 \pm 0.002$ \\
K143R & 143 & $0.599 \pm 0.010$ \\
F145L & 35 & $0.476 \pm 0.021$ \\
P230L & 71 & $0.250 \pm 0.003$ \\
Y257H & 93 & $0.554 \pm 0.029$ \\
D278N & 177 & $0.645 \pm 0.028$ \\
S279F & 152 & $0.289 \pm 0.017$ \\
G307S & 193 & $0.031 \pm 0.001$ \\
S405F & 66 & $0.758 \pm 0.011$ \\
V437I & 37 & $0.796 \pm 0.013$ \\
Y447H & 36 & $0.965 \pm 0.019$ \\
G448E & 35 & $0.407 \pm 0.007$ \\
F449Y & 44 & $0.548 \pm 0.088$ \\
G450E & 163 & $0.517 \pm 0.019$ \\
V456I & 157 & $1.560 \pm 0.018$ \\
G464S & 29 & $0.609 \pm 0.023$ \\
R467K & 38 & $0.471 \pm 0.023$ \\
I471T & 32 & $0.634 \pm 0.015$ \\
Q474K & 35 & $1.601 \pm 0.035$ \\
V488I & 152 & $1.167 \pm 0.032$ \\
Y132F\&K143R & 67 & \\
Y132H\&K143R & 79 & $0.208 \pm 0.014$ \\
Y132F\&F145L & 93 & $0.068 \pm 0.001$ \\
D278N\&G464S & 136 & $0.327 \pm 0.002$ \\
G307S\&G450E & $0.077 \pm 0.008$ \\
\hline & &
\end{tabular}

${ }^{1}$ Yield of purified CaCYP51 protein isolates from one liter of $E$. coli expression culture. ${ }^{2}$ Relative velocity of 1.000 relates to a catalytic turnover number of $0.574 \mathrm{~min}^{-1}$ obtained with the wild type CaCYP51 protein in the CYP51 reconstitution assay. 
Table 3-2. Residual CYP51 activity in the presence of $4 \mu \mathrm{M}$ triazole antifungal

\begin{tabular}{|c|c|c|c|c|c|c|c|c|}
\hline \multirow{3}{*}{$\begin{array}{l}\begin{array}{l}\text { CaCYP51 } \\
\text { substitution }\end{array} \\
\text { Wild type }\end{array}$} & \multicolumn{8}{|c|}{ Residual CYP51 activity $(\%) \pm \mathrm{SD}^{1}$} \\
\hline & \multicolumn{2}{|c|}{ Fluconazole } & \multicolumn{2}{|c|}{ Voriconazole } & \multicolumn{2}{|c|}{ Itraconazole } & \multicolumn{2}{|c|}{ Posaconazole } \\
\hline & 0.11 & \pm 0.09 & 0.11 & \pm 0.01 & 0.03 & \pm 0.02 & 0.66 & \pm 0.02 \\
\hline Y118A & 1.51 & $\pm<0.01$ & 0.28 & \pm 0.04 & 1.30 & \pm 0.59 & 0.46 & \pm 0.35 \\
\hline F126V & 0.09 & \pm 0.06 & 0.19 & \pm 0.06 & 0.22 & \pm 0.05 & 0.10 & \pm 0.01 \\
\hline Y132F & 7.05 & \pm 0.27 & 1.09 & \pm 0.05 & 0.19 & \pm 0.04 & 0.93 & $\pm<0.01$ \\
\hline Y132H & 0.86 & \pm 0.07 & 0.33 & \pm 0.19 & 0.07 & $\pm<0.01$ & 0.18 & \pm 0.06 \\
\hline K143R & 28.14 & \pm 2.04 & 3.12 & \pm 0.27 & 6.27 & \pm 0.51 & 13.09 & \pm 0.02 \\
\hline F145L & 6.71 & \pm 0.15 & 0.37 & \pm 0.11 & 1.99 & \pm 0.04 & 1.37 & \pm 1.21 \\
\hline P230L & 0.05 & \pm 0.03 & 0.13 & \pm 0.12 & 0.06 & \pm 0.01 & 0.21 & \pm 0.05 \\
\hline $\mathrm{Y} 257 \mathrm{H}$ & 3.96 & \pm 0.18 & 0.63 & \pm 0.27 & 1.37 & \pm 0.28 & 6.42 & \pm 0.59 \\
\hline $\mathrm{D} 278 \mathrm{~N}$ & 7.59 & \pm 0.58 & 1.34 & \pm 0.05 & 3.00 & \pm 0.19 & 14.82 & \pm 0.92 \\
\hline S279F & 17.99 & \pm 1.67 & 6.15 & \pm 0.66 & 5.97 & \pm 0.35 & 20.64 & \pm 0.10 \\
\hline G307S & 0.95 & \pm 0.17 & 0.41 & \pm 0.04 & 0.53 & \pm 0.21 & 0.71 & \pm 0.04 \\
\hline $\mathrm{S} 405 \mathrm{~F}$ & 11.56 & \pm 1.41 & 1.94 & \pm 0.12 & 2.73 & \pm 0.13 & 9.38 & \pm 0.53 \\
\hline V437I & 0.22 & \pm 0.06 & 0.02 & $\pm<0.01$ & 0.13 & \pm 0.02 & 0.59 & \pm 0.20 \\
\hline Y447H & 4.34 & \pm 0.51 & 2.07 & \pm 1.04 & 1.45 & \pm 0.06 & 8.19 & \pm 3.58 \\
\hline G448E & 10.46 & \pm 0.20 & 1.89 & \pm 0.61 & 1.75 & \pm 0.26 & 7.89 & \pm 0.29 \\
\hline F449Y & 0.76 & \pm 0.08 & 0.56 & \pm 0.12 & 0.84 & \pm 0.57 & 5.42 & \pm 0.04 \\
\hline G450E & 18.93 & \pm 0.94 & 3.50 & \pm 0.17 & 7.66 & \pm 0.54 & 15.57 & \pm 0.21 \\
\hline V456I & 3.68 & \pm 0.95 & 0.79 & \pm 0.07 & 3.21 & \pm 0.07 & 6.66 & \pm 0.05 \\
\hline G464S & 3.05 & \pm 0.17 & 0.10 & \pm 0.01 & 0.54 & \pm 0.11 & 0.54 & \pm 0.09 \\
\hline $\mathrm{R} 467 \mathrm{~K}$ & 3.34 & \pm 0.61 & 0.52 & \pm 0.18 & 0.45 & \pm 0.17 & 3.62 & \pm 0.09 \\
\hline I471T & 4.35 & \pm 0.33 & 0.92 & \pm 0.05 & 1.21 & \pm 0.01 & 7.71 & \pm 0.58 \\
\hline Q474K & 0.71 & \pm 0.08 & 0.34 & \pm 0.04 & 0.49 & \pm 0.04 & 3.23 & \pm 0.18 \\
\hline V488I & 1.06 & \pm 0.04 & 0.13 & \pm 0.08 & 0.98 & $\pm<0.01$ & 2.11 & \pm 0.14 \\
\hline Y132F\&K143R & 62.04 & \pm 1.21 & 8.86 & \pm 1.09 & 0.23 & \pm 0.13 & 0.86 & \pm 0.11 \\
\hline Y132H\&K143R & 68.81 & \pm 5.68 & 39.59 & \pm 8.63 & 0.70 & \pm 0.03 & 0.74 & \pm 0.05 \\
\hline Y132F\&F145L & 49.10 & \pm 1.76 & 13.58 & \pm 0.19 & 0.91 & \pm 0.05 & 2.64 & \pm 0.08 \\
\hline D278N\&G464S & 18.26 & \pm 0.08 & 4.79 & \pm 0.11 & 3.19 & \pm 0.46 & 14.97 & \pm 1.16 \\
\hline G307S\&G450E & 56.75 & \pm 2.30 & 0.41 & \pm 0.10 & 0.20 & \pm 0.04 & 0.26 & \pm 0.11 \\
\hline
\end{tabular}

${ }^{1}$ Residual activity is expressed as a percentage of the observed CYP51 activity in the absence of triazole antifungals. Each assay was performed in duplicate. Residual CYP51 activities greater than $3 \%$ are indicated in 'bold'. 
susceptibility. However, substitutions such as K143R, S279F, G450E, and G307S\&G450E had at least 3\% residual activity versus all tested azoles. Indeed, since many of these substitutions have been described in fluconazole-resistant isolates, and likely arose after exposure to the antifungal, it is unsurprising that certain substitutions conferred much higher residual activities to fluconazole versus any other tested azole. The double mutations Y132F\&K143R, Y132H\&K143R, Y132F\&145L, and G307S\&G450E, for example, had residual activities of about $50 \%$ or greater with fluconazole, but significantly less activity when exposed to voriconazole, itraconazole, or posaconazole.

In a comparison looking at the $\mathrm{IC}_{50}$ values of each variant $\mathrm{CaCYP} 51$ for fluconazole, voriconazole, itraconazole, and posaconazole, multiple single amino acid substitutions, including K143R, D278N, S279F, S405F, G448E, and G450E conferred $\mathrm{IC}_{50}$ values $\geq 2$ times the $\mathrm{IC}_{50}$ of the wild type (Table 3-3). Furthermore, the single substitutions K143R, D278N, S279F, and G450E had similarly high $\mathrm{IC}_{50}$ values relative to wild type for voriconazole and posaconazole in addition to fluconazole. All double substitutions in CaCYP51 tested also showed high values against fluconazole and voriconazole, ranging from 2 - to $>20$-fold increases in $\mathrm{IC}_{50}$. Interestingly, the azole binding affinity assay did not always reflect the biochemical tolerance observed in the $\mathrm{IC}_{50}$ assay. While the $\mathrm{IC}_{50}$ values of some substitutions implied some degree of biochemical tolerance to fluconazole or other azoles, the $\mathrm{K}_{\mathrm{d}}$ values of these same substitutions revealed relatively higher affinity for the azole drug, in contrast to what might be expected if these substitutions conferred azole resistance through weakening of the azole-CaCYP51 interaction.

These above findings confirm the suspicion that in addition to varied biochemical tolerance and susceptibility to the azoles, mutations in ERG11 can also alter functional efficiency of the enzyme. Altering CaCYP51 function to retain better activity in the presence of the azoles, however, often comes at the cost of worse basal catalytic turnover and possibly at the cost of overall growth fitness to the cell. In light of these recent results, we present our findings of the in vitro susceptibilities of select amino substitutions in CaCYP51 in C. albicans.

\section{Results}

In order to determine the impact of CYP51 amino acid substitutions on in vitro azole susceptibility in C. albicans, we selected several mutations representative of those that led to the greatest biochemical effect on the CYP51 enzyme. When mutant alleles were introduced into the fluconazole-susceptible parent strain SC5314, CaCYP51 amino acid substitutions displayed variable effects on azole drug concentration (Table 3-4). Nine single CYP51 amino acid substitutions and three double CYP51 amino acid substitutions were tested against fluconazole, voriconazole, itraconazole and posaconazole. 
Table 3-3. Triazole antifungal $\mathrm{IC}_{50}$ values obtained with $\mathrm{CaCYP51}$ proteins

\begin{tabular}{|c|c|c|c|c|}
\hline \multirow{2}{*}{$\begin{array}{l}\text { CaCYP51 } \\
\text { substitution }\end{array}$} & \multicolumn{4}{|c|}{$\mathrm{IC}_{50}(\mu \mathrm{M})^{1}$} \\
\hline & Fluconazole & Voriconazole & Itraconazole & Posaconazole \\
\hline Wild type & $0.384 \pm 0.019$ & $0.197 \pm 0.009$ & $0.389 \pm 0.013$ & $0.195 \pm 0.009$ \\
\hline Y118A & $0.194 \pm 0.010$ & $0.202 \pm 0.002$ & $0.170 \pm 0.016$ & $0.206 \pm 0.004$ \\
\hline F126V & $0.278 \pm 0.027$ & $0.341 \pm 0.020$ & $0.206 \pm 0.009$ & $0.304 \pm 0.070$ \\
\hline Y132F & $0.606 \pm 0.066$ & $\mathbf{0 . 4 2 9} \pm 0.012$ & $0.309 \pm 0.003$ & $0.343 \pm 0.020$ \\
\hline $\mathrm{Y} 132 \mathrm{H}$ & $0.220 \pm 0.013$ & $0.197 \pm 0.029$ & $0.150 \pm 0.028$ & $0.195 \pm 0.003$ \\
\hline K143R & $\mathbf{1 . 1 0 0} \pm 0.313$ & $\mathbf{0 . 4 2 4} \pm 0.035$ & $0.450 \pm 0.016$ & $\mathbf{0 . 7 8 9} \pm 0.003$ \\
\hline F145L & $0.450 \pm 0.083$ & $0.231 \pm 0.031$ & $0.300 \pm 0.013$ & $0.359 \pm 0.018$ \\
\hline P230L & $0.334 \pm 0.086$ & $0.241 \pm 0.042$ & $0.265 \pm 0.038$ & $0.238 \pm 0.002$ \\
\hline $\mathrm{Y} 257 \mathrm{H}$ & $0.512 \pm 0.047$ & $0.350 \pm 0.039$ & $0.336 \pm 0.014$ & $\mathbf{0 . 6 1 1} \pm 0.017$ \\
\hline $\mathrm{D} 278 \mathrm{~N}$ & $\mathbf{0 . 8 3 8} \pm 0.027$ & $\mathbf{0 . 5 5 5} \pm 0.004$ & $0.545 \pm 0.044$ & $\mathbf{0 . 9 0 8} \pm 0.020$ \\
\hline S279F & $\mathbf{1 . 0 4 6} \pm 0.325$ & $\mathbf{0 . 4 8 7} \pm 0.002$ & $0.569 \pm 0.121$ & $\mathbf{0 . 9 1 2} \pm 0.016$ \\
\hline G307S & $0.526 \pm 0.211$ & $\mathbf{0 . 4 3 9} \pm 0.018$ & $0.225 \pm 0.007$ & $0.288 \pm 0.042$ \\
\hline $\mathrm{S} 405 \mathrm{~F}$ & $\mathbf{0 . 8 2 0} \pm 0.042$ & $0.362 \pm 0.086$ & $0.350 \pm 0.019$ & $\mathbf{0 . 6 3 7} \pm 0.054$ \\
\hline V437I & $0.281 \pm 0.004$ & $0.304 \pm 0.002$ & $0.293 \pm 0.022$ & $0.319 \pm 0.009$ \\
\hline Y447H & $0.483 \pm 0.018$ & $0.377 \pm 0.055$ & $0.299 \pm 0.039$ & $\mathbf{0 . 4 3 9} \pm 0.135$ \\
\hline G448E & $\mathbf{0 . 7 9 6} \pm 0.087$ & $0.339 \pm 0.011$ & $0.238 \pm 0.013$ & $\mathbf{0 . 5 4 2} \pm 0.051$ \\
\hline F449Y & $0.245 \pm 0.028$ & $0.361 \pm 0.010$ & $0.222 \pm 0.011$ & $0.366 \pm 0.037$ \\
\hline G450E & $\mathbf{1 . 0 7 8} \pm 0.247$ & $\mathbf{0 . 4 2 7} \pm 0.031$ & $0.458 \pm 0.070$ & $\mathbf{0 . 9 0 8} \pm 0.132$ \\
\hline V456I & $0.483 \pm 0.022$ & $\mathbf{0 . 4 6 9} \pm 0.023$ & $0.603 \pm 0.002$ & $\mathbf{0 . 6 0 2} \pm 0.017$ \\
\hline G464S & $0.455 \pm 0.047$ & $0.278 \pm 0.038$ & $0.282 \pm 0.006$ & $0.253 \pm 0.024$ \\
\hline $\mathrm{R} 467 \mathrm{~K}$ & $0.476 \pm 0.080$ & $0.298 \pm 0.049$ & $0.235 \pm 0.018$ & $0.380 \pm 0.011$ \\
\hline $\mathrm{I} 471 \mathrm{~T}$ & $0.600 \pm 0.017$ & $0.318 \pm 0.015$ & $0.218 \pm 0.002$ & $\mathbf{0 . 5 2 2} \pm 0.064$ \\
\hline Q474K & $0.358 \pm 0.008$ & $0.325 \pm 0.002$ & $0.246 \pm 0.006$ & $0.301 \pm 0.017$ \\
\hline V488I & $0.520 \pm 0.057$ & $\mathbf{0 . 4 5 7} \pm 0.016$ & $0.449 \pm 0.016$ & $\mathbf{0 . 4 8 1} \pm 0.052$ \\
\hline Y132F\&K143R & $\mathbf{5 . 8 5 7} \pm 0.524$ & $\mathbf{0 . 5 8 0} \pm 0.099$ & $0.190 \pm 0.002$ & $0.246 \pm 0.024$ \\
\hline Y132H\&K143R & $\mathbf{8 . 4 7 1} \pm 1.271$ & $\mathbf{3 . 0 7 4} \pm 0.042$ & $0.237 \pm 0.007$ & $0.196 \pm 0.007$ \\
\hline Y132F\&F145L & $\mathbf{3 . 8 8 9} \pm 0.077$ & $\mathbf{1 . 1 5 8} \pm 0.231$ & $0.364 \pm 0.023$ & $\mathbf{0 . 4 3 3} \pm 0.102$ \\
\hline D278N\&G464S & $1.243 \pm 0.064$ & $\mathbf{0 . 5 5 2} \pm 0.045$ & $0.403 \pm 0.011$ & $\mathbf{0 . 9 3 0} \pm 0.009$ \\
\hline G307S\&G450E & $4.980 \pm 0.054$ & $\mathbf{0 . 4 3 3} \pm 0.033$ & $0.318 \pm 0.017$ & $0.312 \pm 0.015$ \\
\hline
\end{tabular}

${ }^{1} \mathrm{IC}_{50}$ determinations were performed in duplicate. Mean $\mathrm{IC}_{50}$ values together with standard deviations are shown. Bold values indicate $\mathrm{IC}_{50}$ values that are over 2 -fold greater than those obtained with the wild type protein. 
Table 3-4. CLSI MICs $\left(\mu \mathrm{g} \mathrm{mL} \mathrm{m}^{-1}\right)$ for fluconazole, voriconazole, itraconazole, and posaconazole against strains containing CaCYP51 amino acid substitutions

\begin{tabular}{lcccc}
\hline & \multicolumn{4}{c}{ MIC $\left(\boldsymbol{\mu g} \mathbf{~ m L}^{-1}\right)$} \\
\cline { 2 - 5 } CaCYP51 & Fluconazole & Voriconazole & Itraconazole & Posaconazole \\
\hline None & 1 & $\leq 0.03$ & 0.06 & $\leq 0.03$ \\
Y132F & 8 & 0.5 & 0.25 & $\leq 0.03$ \\
Y132H & 2 & 0.25 & 0.25 & $\leq 0.03$ \\
K143R & 16 & 0.5 & 0.5 & $\leq 0.03$ \\
F145L & 4 & 0.5 & 0.25 & $\leq 0.03$ \\
D278N & 1 & $\leq 0.03$ & 0.125 & $\leq 0.03$ \\
S405F & 4 & 0.25 & 0.25 & $\leq 0.03$ \\
G448E & 8 & 0.25 & 0.25 & 0.06 \\
G450E & 16 & 0.5 & 0.25 & $\leq 0.03$ \\
G464S & 8 & 0.25 & 0.25 & $\leq 0.03$ \\
Y132F, K143R & 32 & 1 & 0.25 & $\leq 0.03$ \\
Y132F, F145L & 32 & 1 & 0.25 & $\leq 0.03$ \\
D278N, G464S & 8 & 0.25 & 0.25 & $\leq 0.03$ \\
\hline
\end{tabular}

${ }^{1}$ Fluconazole-susceptible parent strain SC5314. 
The G450E and K143R amino acid substitutions showed the greatest relative increase in the minimum inhibitory concentration (MIC) for fluconazole, each exhibiting a 16-fold increase in MIC compared to the reference strain SC5314, which lacks any substitution in CYP51. The strains containing the CYP51 single amino acid substitutions Y132F, G448E and G464S also demonstrated resistance to fluconazole, displaying an 8fold increase in MIC over that of SC5314. The overall strongest effect on fluconazole MIC occurred with the double substitutions Y132F\&K143R and Y132F\&F145L, each conferring a 32-fold increase in fluconazole MIC. The D278N amino acid substitution did not appear to alter the fluconazole MIC compared to the wild type, and the $\mathrm{Y} 132 \mathrm{H}$ substitution showed only a 2-fold increase in fluconazole MIC, showing that these individual substitutions have minor effects, if any, on fluconazole MICs.

Against voriconazole, the single substitutions Y132F, K143R, F145L and G450E had the greatest effect on MIC, and the strongest in vitro effect was observed in the Y132F\&K143R and Y132F\&F145L double substitutions. The amino acid substitution with the weakest effect on voriconazole MIC was again D278N $(\leq 0.03 \mathrm{ug} / \mathrm{mL})$. When tested against itraconazole, most of the tested CYP51 substitution strains showed a small increase in MIC, with the strongest single amino acid substitution K143R displaying an

MIC $\left(0.5 \mu \mathrm{g} \mathrm{ml}^{-1}\right)$ greater than that of any of the double amino acid substitutions. While most substitutions causes a small 4-fold increase in MIC to itraconazole, the D278N amino acid substitution only conferred a 2-fold increase in MIC over SC5314. In contrast to the other azoles, posaconazole appeared to be very effective against all CYP51 mutant strains as the MIC to the reference strain and all other strains tested were identical with the exception the strain containing the single G448E substitution, which displayed a slight two-fold increase in MIC over SC5314.

\section{Discussion}

While previous investigations have looked into the contributions of substitutions in CaCYP51 $(122,139)$, the isolated contributions of the D278N and Y132H amino acid substitutions when introduced and tested in C. albicans have not been previously described. Susceptibility testing of these C. albicans strains expressing these substitutions as well as testing of previously constructed CaCYP51 mutant strains is clinically relevant as all these mutations have been associated with fluconazole-resistant clinical isolates.

Furthermore, the recent biochemical and functional findings described by Warrilow et al. in conjunction with our in vitro susceptibility testing in C. albicans provides an important insight that amino acid substitutions in CaCYP51 may confer azole resistance by multiple means. For example, the D278N\&G464S conferred an 8-fold increase in fluconazole MIC over wild type CaCYP51. While the $\mathrm{IC}_{50}$ value reported by Warrilow et al. correlated with the increased fluconazole MIC of these substitutions, the azole ligand binding study showed a relatively low $\mathrm{K}_{d}$ value $(15 \pm 9 \mathrm{nM})$ of the D278N\&G464S CaCYP51 enzyme for fluconazole. This suggests that these two 
substitutions in combination with each other do not have a great effect on fluconazole binding.

This is in stark contrast to the conventional line of thinking that resistanceassociated ERG11 mutations result in CaCYP51 substitutions that reduce the ability of the azole antifungals to bind and inhibit the enzyme. While this remains a likely mechanism of azole resistance for many CaCYP51 substitutions (Y132F\&K143R and Y132H\&K143R, for example, both have $\mathrm{K}_{\mathrm{d}}$ values for fluconazole over 300-fold larger than the wild type enzyme), our susceptibility data with these mutants suggests that azole-resistance conferred by CaCYP51 mutants may be more nuanced than simply altering fluconazole binding. As previously hypothesized, CaCYP51 substitutions could alter interactions between cytochrome P450-reductase, for instance, to affect azole resistance indirectly (140).

The amino acid substitution D278N and Y132H by themselves showed modest increases in azole MIC compared to the wild type. With Y132H, a 2-, 8-, and 4-fold increase in MIC was observed with fluconazole, voriconazole, and itraconazole, respectively. D278N failed to show anything except for a two-fold increase in itraconazole MIC compared to SC5314. Strangely, while Y132H did not show elevated $\mathrm{IC}_{50}$ values to any tested azole compared to the wild type enzyme, D278N had increased $\mathrm{IC}_{50}$ values versus fluconazole, voriconazole, and posaconazole and residual activity versus fluconazole, itraconazole, and posaconazole.

The Y132H CaCYP51 amino acid substitution had previously been shown to increase MIC to fluconazole approximately 4-fold when test in $S$. cerevisiae (122). $\mathrm{Y} 132 \mathrm{H}$ is believed to sit in the ligand binding pocket and alter the interaction with fluconazole and other azoles $(140,206)$. However, the isolated contribution of the D278N amino acid substitution to azole susceptibility has not been reported, and current structural analyses of CaCYP51 do not comment on any significance of the Asp-278 residue in azole binding.

In contrast to the susceptibilities in our tested strains, previous literature showed that adding the D278N amino acid substitution to G464S increased the fluconazole MIC from 4- to 16-fold (139). However, MIC readings were taken at 48 hours, rather than at 24 hours in our study. It is possible the D278N amino acid substitution influences trailing growth and azole tolerance, which might explain the increase in MICs observed at 48 hours, but not 24 hours.

Azole resistance-causing gain-of-function mutations in the transcription factors Tac1, Mrr1, and Upc2 have been shown to also confer conditional fitness defects in $C$. albicans (207-209). Having more than one of these mutations compounds the fitness defect observed, though clinical isolates of $C$. albicans may have compensatory mechanisms by which to regain their original fitness. Given the findings that CaCYP51 substitutions may alter enzyme function, it is conceivable that these amino acid substitutions might also confer a fitness defect on the cell. 
In a competitive growth fitness assay, $C$. albicans strains containing either the wild type CYP51 or mutant CYP51 were grown against the control strain SCADH1 R1A containing the CaSAT1 nourseothricin resistance marker (210). At approximately 24 hours post-inoculation, relative ratios between the test and control strain were fairly constant, regardless of the test strain used, indicative that none of the tested CaCYP51 mutants conferred an observable competitive growth defect after 24 hours (Figure 3-2, unpublished data, personal communication from Joachim Morschhäuser on November 2, 2018). At 48 hours, only strains containing the K143R CaCYP51 amino acid substitution showed a slight drop in ratio of growth at day 0 versus day 2, indicating a trend towards less fit growth compared to the control strain. However, the difference in competitive growth fitness between either wild type strain and the K143R substitution-containing strain was non-significant $(\mathrm{p}=0.12$ and 0.23 between WT A and WT B strains, respectively).

These competitive growth experiments do not indicate that any of our tested substitutions have an immediate impact on cell fitness by themselves. The K143R substitution showed a non-significant decrease in competitive growth compared to the control strain, making it plausible that in longer growth competition experiments, this strain may eventually be outcompeted versus azole-susceptible strains. Because we only tested strains with at most two substitutions in CaCYP51, it remains possible that highly mutated CaCYP51 containing 3 or more amino acid substitutions may display an exacerbated fitness defect driven by poor CaCYP51 function. In a similar vein, it is unknown whether the presence of CaCYP51 mutations in combination with gain-offunction mutations in Tac1, Mrr1, or Upc2 might further decrease fitness in the cell compared to mutations in the ZCFs alone.

CYP51 mutations will likely continue to be a thorn in the side of clinicians and hospitalists prescribing azole antifungals, even with the expected arrival of newer azole antifungal drugs. Therefore, documenting these mutations and characterizing their effects on azole susceptibility, virulence, and fitness are relevant to overcoming azole resistance and ultimately providing better treatment outcomes for invasive fungal infections. As we advance our understanding of the azole antifungal target and effects of amino acid substitutions on drug-target interactions, further investigation should continue to identify key amino acid substitutions involved in the resistance to specific azole drugs. Such work would hopefully serve to provide greater information in the selection of currently available azole antifungals and potentially be useful in the design of future antifungals. 
A

24H Competitive growth fitness of Erg11 mutants

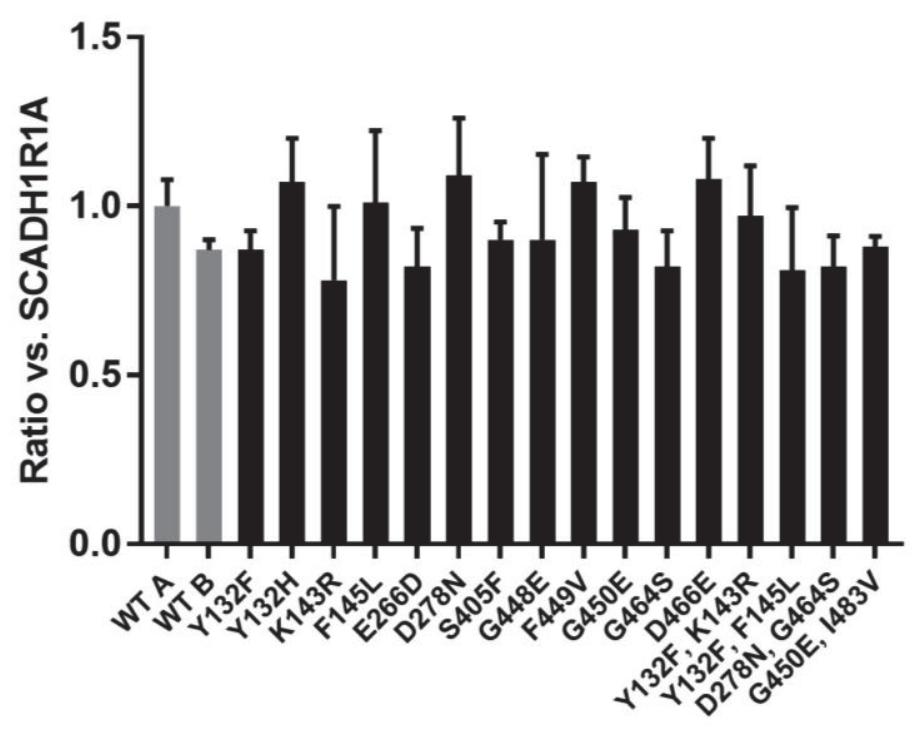

Erg11 amino acid substitutions
B

48H Competitive growth fitness of Erg11 mutants

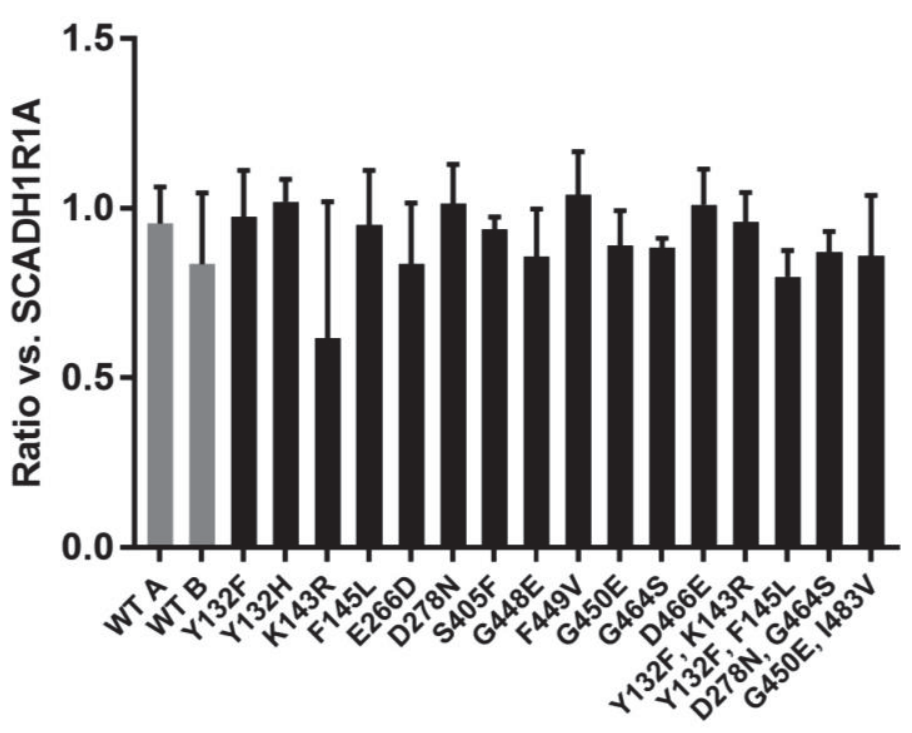

Erg11 amino acid substitution

Figure 3-2. Competive growth fitness testing of Erg11 mutants

(A) 24 hour and (B) 48 hour growth fitness testing of Erg11 mutants versus wild type Erg11. Experiments were performed in triplicate. Data used with permission. Source: Joachim Morschhäuser, personal communication, November 2, 2018. 


\section{Methods}

\section{Construction of C. albicans CYP51 mutant strains}

Strains used in this study are shown in Table 3-5. Strains expressing seven single mutations (Y132F, F145L, K143R, S405F, G448E, G450E, G464S) and three double mutations (Y132F\&F145L, Y132F\&K143R, D278N\&G464S) were selected from aprevious study (139). Two additional strains expressing the Y132H and D278N CYP51 gene mutations were created utilizing the SAT1/FLP cassette described previously (202). Briefly, C. albicans isolate SC5314 was transformed via electroporation with inserts of pSFS2-derived plasmid possessing the SAT1 nourseothricin resistance marker, FLP recombinase, and mutant CaCYP51 genes of interest. The 5' end of the DNA fragment contained either by the Y132H or D278N CYP51 ORF and the 3' end possessed homology downstream of the CaCYP51 stop codon to ensure homologous recombination at the CYP51 gene locus. Successful transformants were screened on yeast-peptonedextrose agar plates containing nourseothricin and confirmed via Southern hybridization.

Genomic DNA from nourseothricin-screened transformants were digested with HindIII restriction enzyme and the resulting DNA digests were loaded on 1\% agarose gels containing ethidium bromide. Digested samples were visualized briefly under ultraviolet light after approximately 2 hours of electrophoresis (120V) to confirm appropriate band separation. Lanes containing digested DNA in the gel was transferred onto a nitrocellulose membrane via vacuum suction and DNA was crosslinked to the membrane through UV exposure. PCR amplification of the genomic DNA from parent isolate SC5314 using primers ERG11_Probe_F (5' AGTTCAATGGTGGTTTTACCTACT - 3') and ERG11_Probe_R (5' ATTTCTGATTGAGTCATCCTAACA - 3') generated a 254 bp product used as a probe for the ERG11 gene locus. Probe was labelled and prepared using the Amersham ${ }^{\mathrm{TM}}$ AlkPhos Direct Labelling and Detection System (GE Life Sciences) and hybridized to the membrane through overnight incubation at $55^{\circ} \mathrm{C}$. Bands containing the hybridized, alkaline phosphatase-labelled probe corresponding to either wildtype ERG11 alleles (2678 bp) or successfully replaced alleles with the $S A T 1 /$ FLP cassette included (4318 bp) or recycled (3119 bp) were visualized with CDP-Star chemiluminescent detection reagent.

To confirm sequence of the replaced ERG11 allele, a DNA fragment containing the ERG11 ORF and approximately 435 bp upstream of the start codon and $229 \mathrm{bp}$ downstream of the ERG11 stop codon was PCR amplified using primers CaERG11_A_(ApaI) (5' - GGGCCCGGGTTATTTGAGAACAGCC - 3') and CaERG11_E_(XhoI) (5' - CTCGAGCCAGTGGACAAAAACCATCA - 3'). The resulting DNA fragment was purified and Sanger sequenced on an ABI Model 3130XL Genetic Analyzer with sequencing primers CaERG11SeqA (5' GCCACCACACCCTATGGCTATT - 3'), CaERG11SeqB (5' - 
Table 3-5. Strains used in Chapter 3

\begin{tabular}{|c|c|c|}
\hline Strains ${ }^{\mathrm{a}}$ & Genotype & Source or reference \\
\hline SC5314 & ERG11-1/ERG11-2 & ATCC \\
\hline \multicolumn{3}{|c|}{ Constructed laboratory strains } \\
\hline 20E1II1B1 & $E R G 11^{Y 132 F} \because: F R T / E R G 11^{Y 132 F}: \because F R T$ & Flowers et al 2015 \\
\hline SCERG11R1S1C1 & $E R G 11^{Y 132 H}:: F R T / E R G 11^{Y 132 H}: \because F R T$ & This study \\
\hline $10 \mathrm{~B} 1 \mathrm{~A} 3 \mathrm{~A}$ & $E R G 11^{K 143 R}: \because F R T / E R G 11^{K 143 R}:: F R T$ & Flowers et al 2015 \\
\hline 2A1A18A & $E R G 11^{F 145 L}: \because F R T / E R G 11^{F / 45 L}: \because F R T$ & Flowers et al 2015 \\
\hline SCERG11R3S3C1 & $E R G 11^{D 278 N} \because \because F R T / E R G 11^{D 278 N} \because \because F R T$ & This study \\
\hline 21C1M1B1 & $E R G 11^{S 405 F} \because: F R T / E R G 11^{S 405 F} \because \because F R T$ & Flowers et al 2015 \\
\hline 20NA11A57A & $E R G 11^{G 448 E}: \because F R T / E R G 11^{G 448 E}:: F R T$ & Flowers et al 2015 \\
\hline $15 \mathrm{~A} 3 \mathrm{~A} 108 \mathrm{~A}$ & $E R G 11^{G 450 E}: \because F R T / E R G 11^{G 450 E}:: F R T$ & Flowers et al 2015 \\
\hline 19A1A1C1 & $E R G 11^{G 4643} \because: F R T / E R G 11^{G 464 S}: \because F R T$ & Flowers et al 2015 \\
\hline 9A14A21A & $E R G 11^{Y 132 F, K 143 R}: \because F R T / E R G 11^{Y 132 F, K 143 R}: \because F R T$ & Flowers et al 2015 \\
\hline $27 \mathrm{~A} 5 \mathrm{~A} 33 \mathrm{~A}$ & $E R G 11^{Y 132 F, F 145 L}: \because F R T / E R G 11^{Y 132 F, F 145 L} \because: F R T$ & Flowers et al 2015 \\
\hline 13A5A57A & $E R G 11^{D 278 N, G 464 S}:: F R T / E R G 11^{D 278 N, G 464 S}:: F R T$ & Flowers et al 2015 \\
\hline
\end{tabular}

${ }^{a}$ All laboratory strains have SC5314 as background. 
TATTTTCACTGCTTCAAGATCT - 3'), CaERG11SeqC (5' CCAAAAGGTCATTATGTTTTAG - 3'), CaERG11SeqD (5' -

CATACAAGTTTCTCTTTTTTCC - 3'), CaERG11SeqE (5' -

CATTTAGGTGAAAAACCTCATT - 3'), and CaERG11SeqF (5' -

TACTCCAGTTTTCGGTAAAGGG - 3').

\section{Susceptibility testing of $C$. albicans CYP51 mutant strains}

Azole minimum inhibitory concentrations (MICs) were determined using the broth microdilution methods as described by the Clinical Laboratory and Standards Institute $(200,201)$. Cells were incubated in 96-well microtiter plates containing Roswell Park Memorial Institute 1640 (RPMI) media and serially-diluted concentrations of either fluconazole, itraconazole, voriconazole, or posaconazole. Concentration for MICs ranged from 0.125 to $64 \mu \mathrm{g} \mathrm{ml}^{-1}$ for fluconazole and 0.03 to $16 \mu \mathrm{g} \mathrm{ml}^{-1}$ for itraconazole, voriconazole, and posaconazole. Measurements were read visually at 24 hours after incubation at $35^{\circ} \mathrm{C}$ for a $50 \%$ reduction in growth from drug-free control wells. MIC measurements were performed in duplicate and the MIC was reported as the higher of two values for all strains tested. In cases where the duplicate MIC values were not identical, the higher of the two MIC values were reported. Overall the MIC values were consistent with $92 \%$ (184/200) MICs within a single dilution of each other for a given strain and drug combination. Additionally, no MICs differed by more than two dilutions for a given strain and drug.

\section{Competitive growth assay}

Competitive growth assays performed by Morschhäuser et al. were performed in triplicate as previously described $(208,210)$. Briefly, each strain was compared to the control strain SCADH1R1A containing the nourseothricin resistance marker CaSAT1. Each test strain and SCADH1R1A were inoculated in liquid YPD media in equal ratios $\left(\mathrm{OD}_{600}=0.002\right)$ and subsequently grown for $26 \mathrm{~h}$ at $30^{\circ} \mathrm{C}$. Cells from the culture were plate at 0 hours, 24 hours and 48 hours onto YPD-agar plates and YPD-agar with either $200 \mu \mathrm{g} / \mathrm{mL}$ or $15 \mu \mathrm{g} / \mathrm{mL}$ nourseothricin. Colonies on YPD with $200 \mu \mathrm{g} / \mathrm{mL}$ nourseothricin represented the nourseothricin-resistant SCADH1R1A, and smaller colonies on YPD with $15 \mu \mathrm{g} / \mathrm{mL}$ nourseothricin represented the nourseothricin-sensitive test strain. Colonies were counted to determine the percentage in the co-culture at each timepoint. 


\section{CHAPTER 4. THE ROLE OF ERG3 MUTATIONS TO AZOLE RESISTANCE AND ERG11 MUTABILITY IN C. ALBICANS}

\section{Introduction}

Candida albicans is a commensal organism and opportunistic pathogen that can cause a wide array of infections, ranging from superficial mucosal infection to lifethreatening invasive disease. In the United States, Candida remains a common cause of healthcare-associated infection $(12,183)$. In particular, mortality associated with Candida bloodstream infections remains high, and proper treatment of the infection increases hospitalization costs and length of stay considerably $(59,68,182)$. Additionally, mucosal infections such as vaginal candidiasis and, in immunocompromised patients, oropharyngeal candidiasis are common infections in which C. albicans remains one of the most frequent causes of disease (211-213).

The azole antifungal drugs are commonly used in treatment and prophylaxis of candidiasis. By inhibiting 14 $\alpha$-lanosterol demethylase, an enzyme critical to the production of the important yeast cellular membrane sterol ergosterol, the azoles inhibit cell growth. The primarily fungistatic action of the azole antifungals is thought to be linked the accumulation of the toxic sterol 14 $\alpha$-methylergosta-8,24(28)-dien-3 $\beta, 6 \alpha$-diol within the cell $(71,72)$.

C. albicans is able to avoid the inhibitory effects of the azole antifungals through several mechanisms. Commonly, C. albicans isolates resistant to the azole have increased expression of genes encoding for efflux pumps that reduce the intracellular concentrations of azoles inside the cell, such as the ABC transporters Cdr1 and Cdr2 and the MFS transporter Mdr1 $(78,79,99)$. Changes to the azole drug target $14 \alpha$-lanosterol demethylase brought about by nucleotide changes in its encoding gene ERG11 can also reduce the effectiveness of the azole antifungals to bind or otherwise inhibit the activity of the enzyme $(107,139,140)$. Additionally, increases in the amount of azole target through upregulation of ERG11 expression can also overcome to some degree the presence of the azole antifungals inside the cell $(106,214,215)$.

The above mechanisms are among the most commonly reported determinants of azole resistance in clinical isolates, however, it has been less commonly reported that alterations in the $C$. albicans sterol biosynthesis pathway mediated by mutations in $E R G 3$ can also confer azole resistance. ERG3 encodes for the 386 amino acid length C-5 sterol desaturase in Candida albicans a component essential to the production of ergosterol (71, 216). It had been shown that defective Erg3 confers azole-resistance in C. albicans by avoiding accumulation of toxic sterol inside the cell and that Erg3-defective cells had significantly different sterol profiles $(72,144,149)$. This signifies that overall without the presence of a functional C-5 sterol desaturase, the $C$. albicans cells utilizes alternate sterol biosynthesis pathways in order to survive. Since the toxic $14 \alpha$-methylergosta8,24(28)-dien-3 $\beta, 6 \alpha$-diol required Erg3 for its production, mutant isolates lacking functional C-5 sterol desaturase would not accumulate the toxic sterol even in the 
presence of the Erg11-inhibiting azoles (Figures 4-1 and 4-2). Therefore, isolates lacking in or defective for Erg3 activity were rendered completely resistant to the azole antifungal class $(142,148)$.

Previously, we had characterized a collection of predominantly azole-resistant clinical isolates of $C$. albicans (115). Based on this data, we identified several members among this collection which could not be explained by common mechanisms of resistance in C. albicans. While mutations in ERG3 can confer azole resistance to clinical isolates, their rarely reported frequency and debate over attenuated virulence in these isolates makes the clinical significance of ERG3 mutations questionable. However, Erg3 is inexorably tied to Erg11 as both proteins are crucial to the biosynthesis of ergosterol (147). Moreover, a recent finding by Warrilow et al. demonstrated that mutations in ERG11 can have variable effects on 14a-lanosterol demethylase activity, and while amino acid substitutions in Erg11 conferred azole resistance, they often came at a cost to the catalytic turnover of the enzyme (141).

Since loss of demethylase activity by treatment with the azoles triggers accumulation of the toxic sterol intermediates in C. albicans, it is plausible that some ERG11 mutations may result in markedly poorer demethylase activity and therefore may accumulate higher levels of toxic sterol intermediates. Furthermore, since the toxic sterol production is dependent on Erg3 activity, we believe that mutations in Erg3 that alter activity or substrate specificity of the enzyme may alleviate accumulation of toxic sterols. Therefore, it is possible that mutations in ERG3 allows conditional ERG11 mutations in $C$. albicans to exist. In other words, ERG3 mutants may increase the mutability of $E R G 11$, thereby increasing the opportunistic potential to develop azole-resistance through $E R G 11$ mutation. In this study we identified the mutations in ERG3 across our collection of clinical isolates and posit that mutations in ERG3 may be more common than popularly believed and may play a larger role in azole resistance than previously believed.

\section{Results}

\section{Frequency of Erg11 and Erg3 amino acid substitutions in C. albicans clinical isolates}

Sequencing of ERG11 and ERG3 within 57 fluconazole-resistant (MIC $\geq 8$ $\mu \mathrm{g} / \mathrm{mL})$ clinical C. albicans isolates revealed 51 of $57(89 \%)$ isolates with Erg11 amino acid substitutions (Table 4-1). Overall, 20 of 57 (35\%) clinical isolates possessed amino acid substitutions in Erg3. In particular, a $\mathrm{C}>\mathrm{T}$ nucleotide change at position 1052 in ERG3 corresponding to an $\mathrm{A} 351 \mathrm{~V}$ amino acid substitution appeared either alone or together with another Erg3 substitution in 18 (90\%) of all ERG3 mutant isolates. Among the 51 isolates with Erg11 amino acid substitutions, concomitant Erg3 amino acid substitutions occurred in 19 (37\%) isolates (Table 4-1). Interestingly, all Erg3 mutants containing either a homozygous or heterozygous A351V amino acid substitution possessed at least one substitution in Erg11, and 15 of $18(83 \%)$ of those isolates 


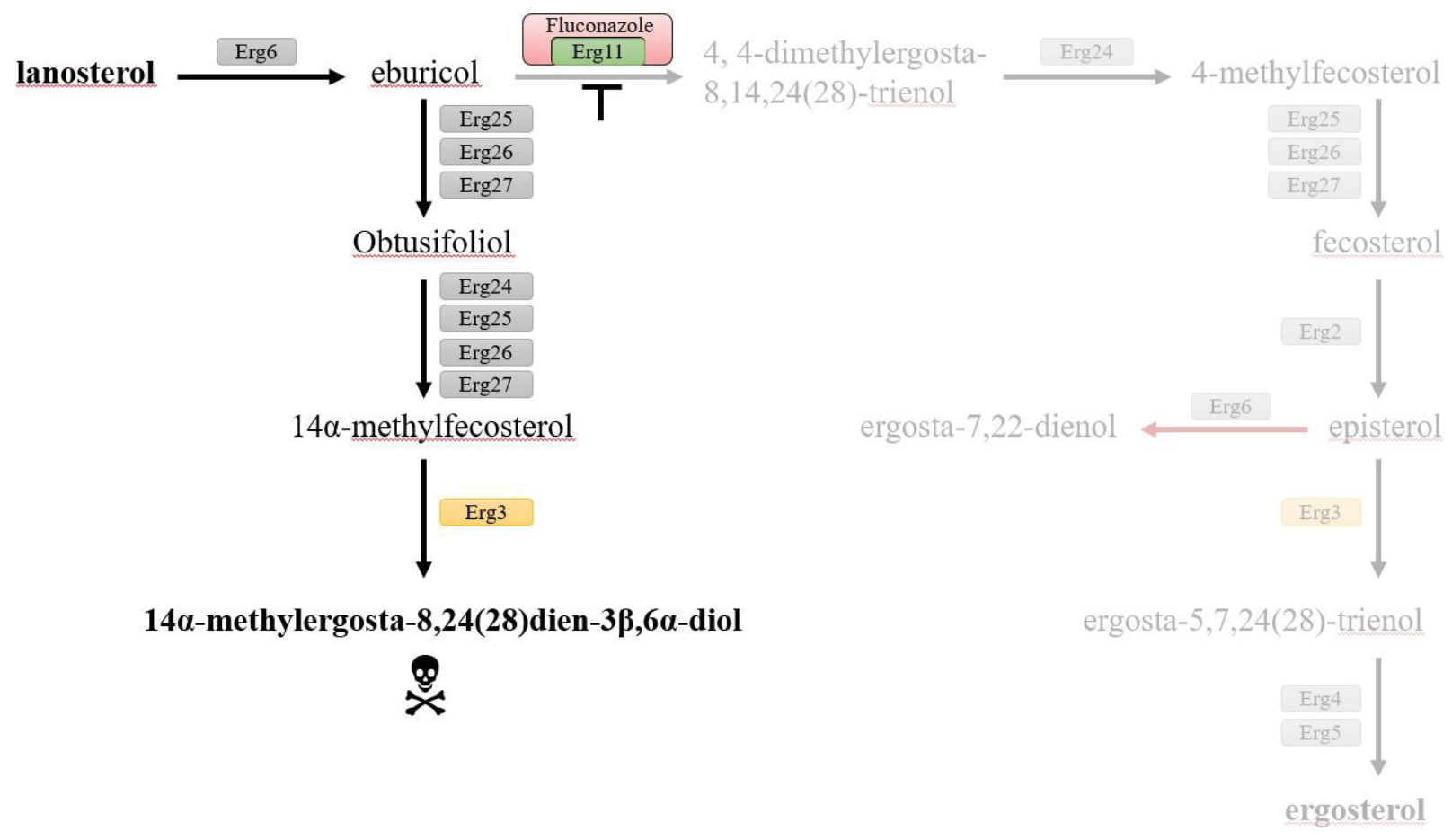

Figure 4-1. Normal ergosterol biosynthesis pathway inhibition by the azole antifungals in $C$. albicans

Simplified representation of the later stages of ergosterol biosynthesis converting lanosterol to ergosterol is interrupted with fluconazole treatment. Fluconazole is represented by the light red rectangle. The azole target enzyme is shown in green. Grayed areas represent blocked biosynthesis pathways. T-shaped representation of pathway inhibition is shown at inhibition sites. A skull and crossbones represents the accumulation of toxic sterol. 


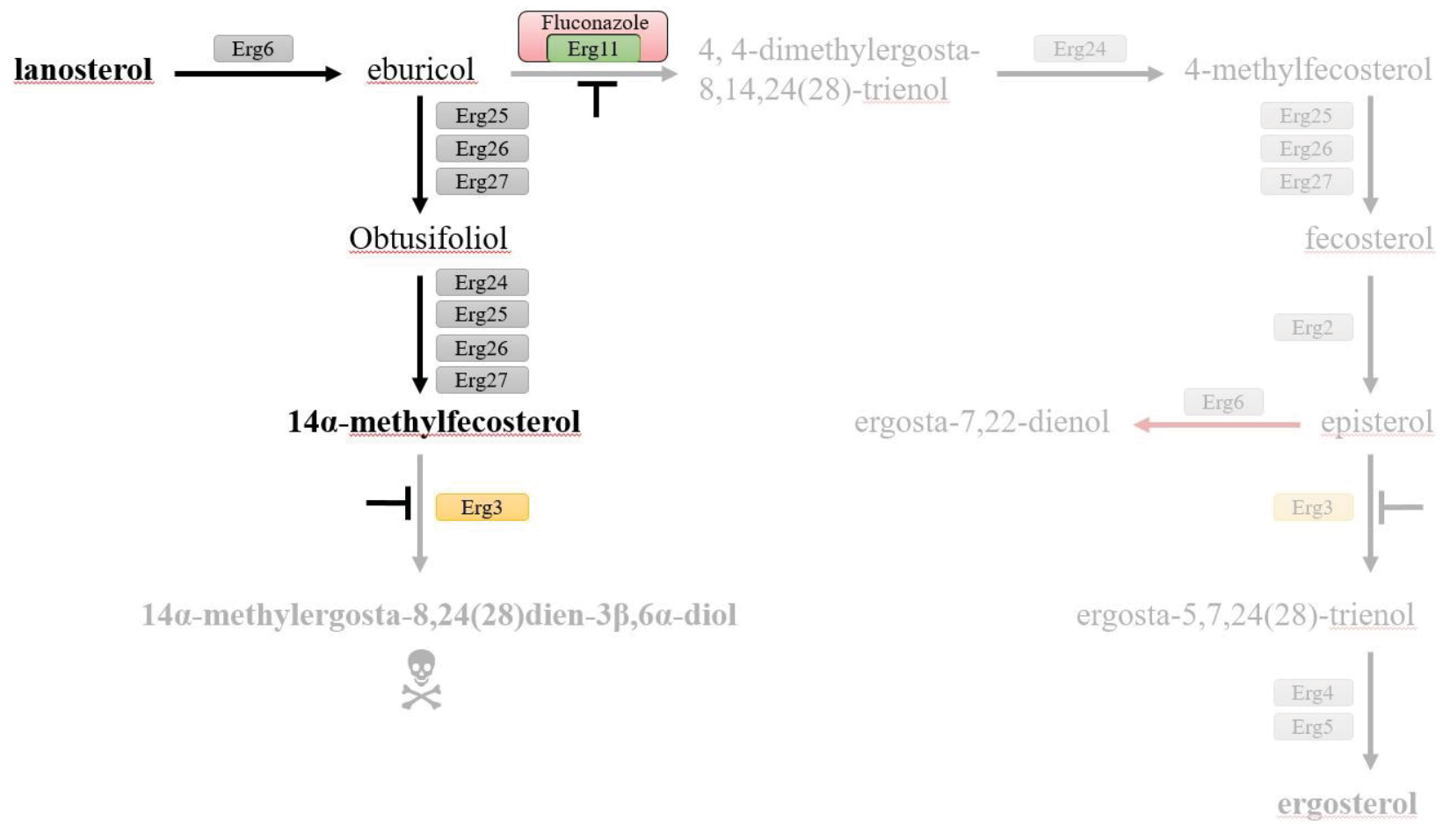

Figure 4-2. Ergosterol biosynthesis pathway inhibition by the azole antifungals in C. albicans with defective $\mathbf{C}-5$ sterol desaturase

Simplified representation of the later stages of ergosterol biosynthesis converting lanosterol to ergosterol is interrupted with fluconazole treatment, but the toxic azole cannot be produced due to defective Erg3. Fluconazole is represented by the light red rectangle. The azole target enzyme is shown in green. Grayed areas represent blocked biosynthesis pathways. T-shaped representation of pathway inhibition is shown at inhibition sites. Erg3 is represented in yellow. 
Table 4-1. Fluconazole MIC, ergosterol content, and mutations in ERG11 and ERG3 in clinical isolates of $C$. albicans

\begin{tabular}{|c|c|c|c|c|}
\hline Isolate & $\begin{array}{l}\text { MIC }_{\text {FLU }} \\
(\mu \mathrm{g} / \mathrm{mL})\end{array}$ & $\%$ Ergosterol & ERG11 mutation ${ }^{a}$ & $\begin{array}{c}\text { ERG3 amino acid } \\
\text { substitution }^{\mathrm{a}}\end{array}$ \\
\hline 11 & 8 & 75.5 & E266D, V488I & $\mathrm{A} 351 \mathrm{~V}$ \\
\hline 12 & 32 & 73.5 & K143R & None \\
\hline 13 & 32 & 54.1 & K143R & None \\
\hline 14 & 16 & 72.3 & K143R & None \\
\hline 15 & 16 & 64.2 & E266D, V488I & $\mathrm{A} 351 \mathrm{~V}(\mathrm{~h}), \mathrm{A} 353 \mathrm{~T}(\mathrm{~h})$ \\
\hline 16 & 8 & 27.7 & Q21R (h) & A351V \\
\hline 18 & 32 & 49.1 & S405F & None \\
\hline 19 & 32 & 47.8 & S405F & None \\
\hline 20 & 64 & 52.3 & $\mathrm{~S} 405 \mathrm{~F}$ & None \\
\hline 21 & 32 & 45.0 & A114V, E266D, H283R & A351V (h), A353T (h) \\
\hline 23 & 32 & 53.2 & $\mathrm{I} 483 \mathrm{~V}, \mathrm{G} 450 \mathrm{E}$ & A351V (h) \\
\hline 24 & 16 & 41.2 & G450E & None \\
\hline 25 & 16 & 53.6 & D446E & None \\
\hline 26 & 16 & 47.5 & G307S, G448R & A351V (h) \\
\hline 27 & 16 & 53.9 & E266D, V488I & A351V (h), A353T (h) \\
\hline 28 & 64 & 50.7 & E266D, V488I & A351V (h), A353T (h) \\
\hline 29 & $>64$ & 58.1 & Y132F, F145L & A351V (h), A353T (h) \\
\hline 30 & 32 & 51.1 & Y132F, H283Y & None \\
\hline 31 & $>64$ & 66.9 & G448E & None \\
\hline 32 & 8 & 71.0 & G464S & None \\
\hline 33 & 32 & 60.5 & F145L, E266D & None \\
\hline 34 & 64 & 40.4 & G450E & None \\
\hline 35 & 16 & 75.5 & D446E & None \\
\hline 36 & 64 & 76.0 & None & None \\
\hline 37 & 64 & 68.1 & None & None \\
\hline 38 & $>64$ & 74.0 & G464S & None \\
\hline 39 & $>64$ & 70.0 & G464S & None \\
\hline 40 & $>64$ & 70.0 & F449V & None \\
\hline 41 & 64 & 55.7 & $\mathrm{~S} 405 \mathrm{~F}$ & None \\
\hline 42 & 64 & 58.8 & G464S & None \\
\hline 43 & 8 & 74.0 & D278N, G464S & None \\
\hline 44 & $>64$ & 60.9 & $\mathrm{D} 446 \mathrm{E}$ & None \\
\hline 45 & 64 & 76.0 & E266D, G464S & A351V \\
\hline 46 & 64 & 45.7 & G307S, L403F, G448R & A351V (h) \\
\hline 47 & $>64$ & 73.9 & G450E & A351V (h) \\
\hline 48 & 32 & 70.5 & Y132F & L195V (h), A351V (h) \\
\hline 49 & $>64$ & 77.7 & V437I, Y447S & $\mathrm{H} 28 \mathrm{Y}(\mathrm{h})$ \\
\hline 50 & $>64$ & 72.0 & Y132F, K143R & None \\
\hline 51 & $>64$ & 57.5 & D278N, G464S & None \\
\hline 54 & 32 & 61.5 & G307S, G450E & A351V (h) \\
\hline
\end{tabular}


Table 4-1. (Continued)

\begin{tabular}{ccccc}
\hline Isolate & $\begin{array}{c}\text { MIC } \\
(\boldsymbol{\mu g} / \mathbf{m L})\end{array}$ & $\begin{array}{c}\text { \% Ergosterol } \\
\text { ERG11 mutation }\end{array}$ & $\begin{array}{c}\text { ERG3 amino acid } \\
\text { substitution }^{1}\end{array}$ \\
\hline 55 & $>64$ & 66.0 & None & None \\
56 & $>64$ & 74.2 & None & None \\
58 & $>64$ & 80.9 & None & None \\
59 & $>64$ & 71.7 & Y132F, T229A, F449I & A351V (h) \\
60 & $>64$ & 0.8 & None & W131* \\
61 & $>64$ & 46.6 & Y132F, K143R & None \\
62 & 32 & 69.8 & D278N, G464S & None \\
63 & 32 & 70.3 & G450E & None \\
64 & 32 & 52.6 & G450E & None \\
65 & 64 & 74.5 & G448E & None \\
66 & 64 & 78.3 & G448E & None \\
67 & $>64$ & 82.4 & G448E & None \\
68 & $>64$ & 60.2 & Y132F, E266D, I471M, & None \\
& & & V488I & None \\
69 & 64 & 76.0 & G448E & A351V \\
70 & 32 & 71.5 & M258L, G464S (h) & A351V (h) \\
71 & $>64$ & 71.0 & Y132F, V437I, F449L & A351V (h) \\
72 & 16 & 69.5 & A114V, D153E, E266D, & \\
& & & G450E & \\
\hline
\end{tabular}

${ }^{1}(\mathrm{~h})$ indicated a heterozygous amino acid substitution. Pink highlighted rows indicate $E R G 3^{\mathrm{A} 351 \mathrm{~V}}$ mutant clinical isolates. 
contained more than one amino acid substitution in Erg11. By comparison, among isolates lacking Erg3 amino acid substitutions, 32 of 38 (84\%) had at least one Erg11 substitution and only $8(21 \%)$ isolates possessed multiple Erg11 amino acid substitutions. The frequency with which the A351V Erg3 amino acid substitution coincided with more than one Erg11 amino acid substitution in clinical isolates and the relatively lower frequency of multiple Erg11 amino acid substitutions in isolates lacking the A351V substitution suggested that this substitution may possibly be associated with hypermutability of ERG11.

Incidentally, we also discovered four novel Erg3 substitutions in select isolates (Table 4-1). Isolate 49 contained a heterozygous H28Y amino acid substitution, and the Erg3 of isolate 60 was truncated due to mutation encoding a premature stop codon after only 130 amino acids. The L195V and A353T substitutions only appeared heterozygously and only in combination with the heterozygous A351V substitution in isolates 48 and isolates 15, 21, 27, 28, and 29, respectively.

\section{Sterol content in clinical isolates}

In an effort to determine whether substitutions in Erg3 altered sterol content, whole cell membrane sterol contents from the clinical isolates (Table 4-2). Ergosterol, normally the major sterol component of the fungal cell membrane, made up on average approximately $62 \%$ (median: $67.5 \%$, range: $0.8 \%-82.4 \%$ ) of whole cell membrane sterol content in our fluconazole-resistance clinical isolates. In isolates lacking Erg3 substitutions the average ergosterol content was approximately $64 \%$ (median: $68.5 \%$, range: $40.4 \%-82.4 \%$ ) and in the Erg3 mutants containing the A351V substitution, the cell membrane ergosterol content was approximately $60.4 \%$ (median: $62.9 \%$, range: $27.7 \%-76 \%$ ). Thus, the presence or absence of the A351V substitution in Erg3 did not seem to affect ergosterol content in the cell under normal growth conditions.

The average ergosterol content when looking at all isolates containing Erg3 substitutions of any sort was considerably skewed lower $(58.3 \%)$ due to the only $0.8 \%$ ergosterol content in isolate 60, containing a nonsense mutation (W131*) in ERG3. This premature termination of the Erg3 protein very likely would result in a dysfunctional or nonfunctional enzyme unable to produce significant amount of ergosterol, possibly explaining the isolate's low ergosterol content (Figures 4-3 and 4-4).

\section{Allelic replacement of $E R G 3$}

\section{The A351V amino acid substitution in Erg3 does not directly affect in vitro fluconazole susceptibility}

To determine whether the A351V mutation had a direct effect on azole resistance, we created an $\operatorname{erg} 3 \Delta / \Delta$ strain as well as an $E R G 3^{\mathrm{A} 351 \mathrm{~V}}$ mutant in the azole-susceptible 
Table 4-2. Percent total cell membrane sterol content composition in $C$. albicans clinical isolates

\begin{tabular}{|c|c|c|c|c|c|c|c|c|}
\hline $\begin{array}{l}\text { Strain } \\
\text { (C. albicans) }\end{array}$ & Lanosterol & Eburicol & Fecosterol & Episterol & Ergosterol $^{1}$ & $\begin{array}{c}\text { Ergosta- } \\
7,22 \text {-dienol }\end{array}$ & $\begin{array}{l}\text { 14-methyl } \\
\text { fecosterol }\end{array}$ & 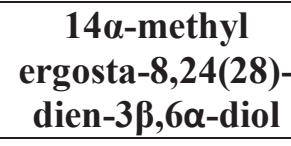 \\
\hline 1 & 1.3 & & 0.5 & 1.0 & 91.4 & & & \\
\hline 2 & 4.2 & 0.5 & 2.5 & 6.2 & 65.8 & 1.1 & & \\
\hline 3 & 3.4 & 0.1 & 1.1 & 2.1 & 76.7 & & & \\
\hline 4 & 0.7 & & 1.6 & 2.3 & 69.1 & & & \\
\hline 5 & 6.3 & 0.7 & 5.0 & 6.5 & 55.0 & & & \\
\hline 6 & 8.3 & 10.4 & 1.1 & 0.5 & 58.9 & 0.2 & & 0.4 \\
\hline 7 & 2.1 & 0.1 & 1.8 & 5.4 & 74.1 & & & \\
\hline 8 & 2.0 & & 3.5 & 8.6 & 55.7 & 6.3 & & \\
\hline 9 & 7.2 & 0.5 & 4.9 & 9.0 & 46.4 & 1.6 & & \\
\hline 10 & 11.1 & 3.6 & 1.8 & & 62.5 & & & \\
\hline 11 & 4.2 & 0.3 & 1.1 & 2.5 & 75.5 & 0.4 & & \\
\hline 12 & 6.1 & 3.2 & 0.8 & 1.7 & 73.5 & & & \\
\hline 13 & 11.8 & 9.6 & 2.5 & 3.1 & 54.1 & & & \\
\hline 14 & 5.0 & 2.4 & 2.3 & 4.5 & 72.3 & & & 0.0 \\
\hline 15 & 3.6 & 0.8 & 2.7 & 6.3 & 64.2 & 0.4 & & \\
\hline 16 & 18.0 & 5.2 & 5.1 & 12.8 & 27.7 & 0.4 & & 0.7 \\
\hline 17 & 3.1 & 0.2 & 1.7 & 3.0 & 75.2 & & & \\
\hline 18 & 14.5 & 19.0 & 0.7 & 1.2 & 49.1 & & 2.8 & 1.9 \\
\hline 19 & 17.2 & 19.6 & 0.6 & 0.7 & 47.8 & & 3.1 & 2.0 \\
\hline 20 & 22.1 & 6.7 & 1.7 & 2.5 & 52.3 & & 1.1 & 0.5 \\
\hline 21 & & & & 1.9 & 45.0 & & & 4.3 \\
\hline 22 & 8.1 & 2.8 & 2.0 & 4.1 & 60.5 & & 1.0 & \\
\hline 23 & 25.6 & & 0.4 & 1.8 & 53.2 & & 3.6 & \\
\hline
\end{tabular}


Table 4-2. (Continued)

\begin{tabular}{|c|c|c|c|c|c|c|c|c|}
\hline $\begin{array}{l}\text { Strain } \\
\text { (C. albicans) }\end{array}$ & Lanosterol & Eburicol & Fecosterol & Episterol & Ergosterol & $\begin{array}{c}\text { Ergosta- } \\
7,22 \text {-dienol }\end{array}$ & $\begin{array}{l}\text { 14-methyl } \\
\text { fecosterol }\end{array}$ & $\begin{array}{c}14 \alpha \text {-methyl } \\
\text { ergosta-8,24(28)- } \\
\text { dien-3 } \beta, 6 \alpha \text {-diol }\end{array}$ \\
\hline 24 & 29.5 & 19.2 & 0.2 & 1.5 & 41.2 & 1.3 & 1.2 & \\
\hline 25 & 3.0 & & 3.2 & 6.0 & 53.6 & & & \\
\hline 26 & 20.8 & 14.4 & & 0.7 & 47.5 & & 3.4 & 6.4 \\
\hline 27 & 13.7 & 1.6 & 3.9 & 7.5 & 53.9 & & & \\
\hline 28 & 10.2 & 1.0 & 4.3 & 10.3 & 50.7 & & & \\
\hline 29 & 12.5 & 2.2 & 4.7 & 5.0 & 58.1 & & & \\
\hline 30 & 22.4 & 2.9 & 1.3 & 2.6 & 51.1 & & & \\
\hline 31 & 9.0 & 12.1 & 0.4 & 1.4 & 66.9 & & 1.2 & 1.3 \\
\hline 32 & 5.7 & 0.2 & 1.9 & 3.8 & 71.0 & & & \\
\hline 33 & 13.2 & 1.1 & 1.5 & 4.2 & 60.5 & & & \\
\hline 34 & 15.7 & 8.0 & 1.5 & 4.6 & 40.4 & 0.4 & 1.5 & \\
\hline 35 & 1.1 & & 2.0 & 4.7 & 75.5 & & & \\
\hline 36 & 1.0 & & 1.2 & 3.0 & 76.0 & & & \\
\hline 37 & 1.4 & & 3.4 & 5.0 & 68.1 & & & \\
\hline 38 & 2.4 & & 1.6 & 3.2 & 74.0 & & & \\
\hline 39 & 3.1 & & 1.9 & 4.4 & 70.0 & & & \\
\hline 40 & 5.8 & 4.6 & 0.3 & 1.4 & 70.0 & & & \\
\hline 41 & 6.8 & 0.3 & 2.2 & 4.2 & 55.7 & & & \\
\hline 42 & 7.6 & 1.9 & 1.2 & 4.6 & 58.8 & 0.7 & & \\
\hline 43 & 5.2 & 0.3 & 0.5 & 1.2 & 74.0 & & & \\
\hline 44 & 4.3 & 0.7 & 2.9 & 7.9 & 60.9 & 0.7 & & \\
\hline 45 & 2.9 & 0.5 & 0.6 & 2.2 & 76.0 & & & \\
\hline 46 & 24.9 & 11.0 & 1.0 & 1.0 & 45.7 & & 0.9 & \\
\hline 47 & 5.6 & 3.7 & 0.4 & 0.9 & 73.9 & & & \\
\hline
\end{tabular}




\section{Table 4-2. (Continued)}

\begin{tabular}{|c|c|c|c|c|c|c|c|c|}
\hline $\begin{array}{l}\text { Strain } \\
\text { (C. albicans) }\end{array}$ & Lanosterol & Eburicol & Fecosterol & Episterol & Ergosterol & $\begin{array}{c}\text { Ergosta- } \\
\text { 7,22-dienol }\end{array}$ & $\begin{array}{l}\text { 14-methyl } \\
\text { fecosterol }\end{array}$ & 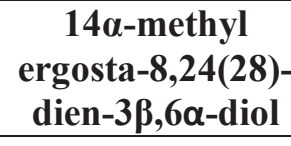 \\
\hline 48 & 7.4 & 3.2 & 1.2 & 2.6 & 70.5 & \multirow{12}{*}{0.9} & \multirow{12}{*}{1.3} & \\
\hline 49 & 6.0 & 1.6 & 0.8 & 1.4 & 77.7 & & & \\
\hline 50 & 13.0 & 3.3 & 0.6 & 0.2 & 72.0 & & & \\
\hline 51 & 13.1 & 1.3 & 0.8 & 2.1 & 57.5 & & & \\
\hline 52 & 3.5 & 0.4 & 2.4 & 5.1 & 67.5 & & & \\
\hline 53 & 2.6 & 0.6 & 1.0 & 2.1 & 73.9 & & & \\
\hline 54 & 7.6 & 20.3 & 0.5 & & 61.5 & & & \\
\hline 55 & 1.7 & 0.1 & 3.7 & 4.1 & 66.0 & & & \\
\hline 56 & 1.3 & 0.2 & 3.6 & 5.1 & 74.2 & & & \\
\hline 57 & 1.4 & 3.7 & 1.7 & 3.2 & 68.8 & & & \\
\hline 58 & 1.2 & 1.0 & 1.3 & 2.4 & 80.9 & & & \\
\hline 59 & 2.0 & 0.4 & 1.9 & 3.9 & 71.7 & & & \\
\hline 60 & 0.9 & & 2.0 & 4.8 & 0.8 & 62.1 & & \\
\hline 61 & 15.9 & 11.0 & 0.7 & 1.5 & 46.6 & & & \\
\hline 62 & 8.5 & 2.1 & 0.5 & 1.5 & 69.8 & & & \\
\hline 63 & 5.5 & 3.8 & & & 70.3 & & & \\
\hline 64 & 11.6 & 11.7 & 1.0 & 2.6 & 52.6 & & & \\
\hline 65 & 4.0 & 1.4 & 1.2 & 2.9 & 74.5 & & & \\
\hline 66 & 5.2 & 3.3 & & 1.4 & 78.3 & & & \\
\hline 67 & 3.0 & 1.0 & & 1.3 & 82.4 & & & \\
\hline 68 & 10.0 & 6.2 & 1.3 & 3.2 & 60.2 & & & \\
\hline 69 & 3.9 & 1.6 & 0.8 & 2.0 & 76.0 & & & \\
\hline
\end{tabular}




\section{Table 4-2. (Continued)}

\begin{tabular}{|c|c|c|c|c|c|c|c|c|}
\hline $\begin{array}{l}\text { Strain } \\
\text { (C. albicans) }\end{array}$ & Lanosterol & Eburicol & Fecosterol & Episterol & Ergosterol & $\begin{array}{c}\text { Ergosta- } \\
\text { 7,22-dienol }\end{array}$ & $\begin{array}{l}\text { 14-methyl } \\
\text { fecosterol }\end{array}$ & 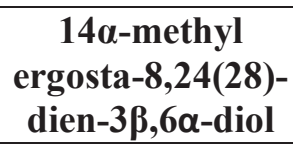 \\
\hline 70 & 3.2 & 0.6 & 1.0 & 2.3 & 71.5 & & & \\
\hline 71 & 6.8 & 4.7 & & 0.9 & 71.0 & & & \\
\hline 72 & 9.2 & 5.7 & & 0.8 & 69.5 & & & \\
\hline
\end{tabular}

${ }^{1}$ Percent ergosterol content is reflected in the center of the table and colored to indicate increased (greener) or decreased (redder) total ergosterol content. Isolate 60 , whose sterol content closely resembles a typical erg $3 \Delta$ sterol profile that lacks ergosterol, is highlighted in yellow. Blank entries under each sterol heading indicates there was none of that particular sterol detected for each isolate. 


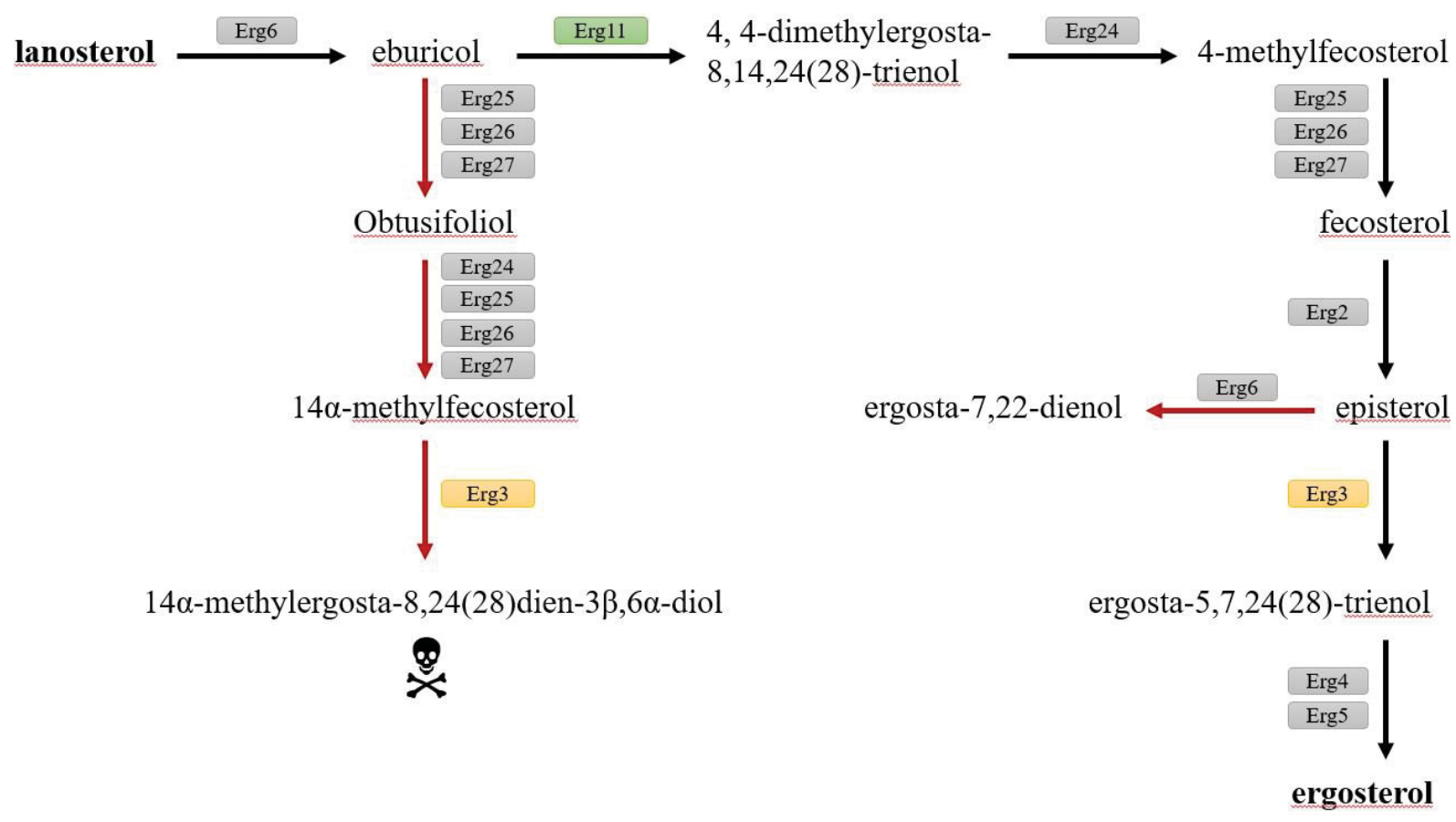

Figure 4-3. Normal ergosterol biosynthesis pathway in $C$. albicans

Simplified representation of the later stages of ergosterol biosynthesis converting lanosterol to ergosterol. The azole target enzyme Erg11 is shown in green. Red arrows represent alternate biosynthesis pathways. T-shaped representation of pathway inhibition is shown at inhibition sites. 


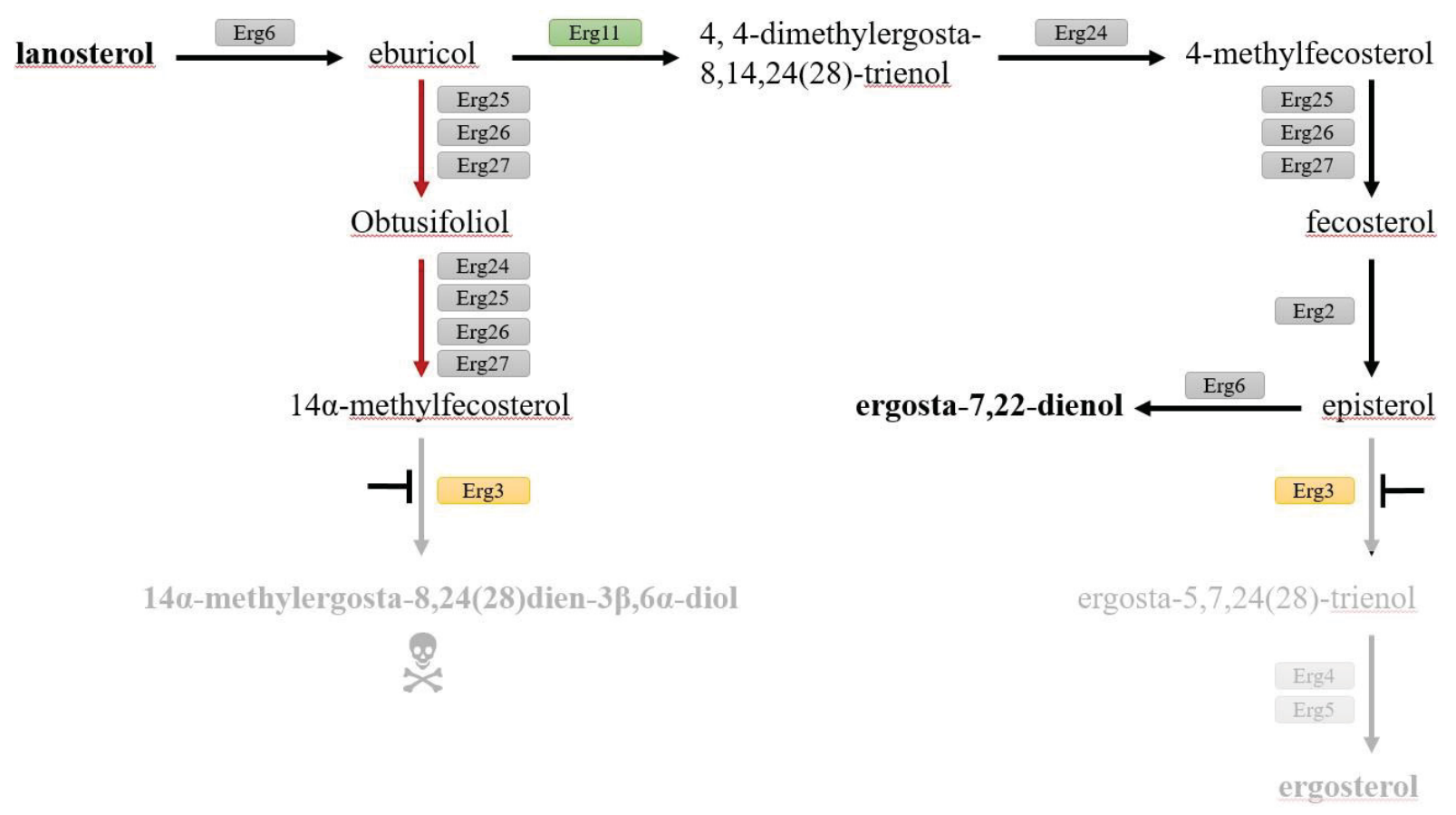

Figure 4-4. Alternative sterol biosynthesis pathway in $C$. albicans cells with defective C-5 sterol desaturase

Simplified representation of the later stages of ergosterol biosynthesis converting lanosterol to ergosterol shows that ergosterol biosynthesis cannot be completed without Erg3 activity. The azole target enzyme Erg11 is shown in green. Erg3 is shown in yellow. Red arrows represent alternate biosynthesis pathways. T-shaped representation of pathway inhibition is shown at inhibition sites. Grayed areas represent blocked biosynthesis pathways. 
isolate SC5314. Deletion of the $E R G 3$ gene from both native loci resulted in complete resistance to fluconazole (Table 4-3), consistent with the traditional ERG3 resistance phenotype. However, when the ERG3 allele containing the nucleotide mutation corresponding to the $\mathrm{A} 351 \mathrm{~V}$ amino acid substitution was replaced again in both native loci, the strains appeared to have no direct effect on fluconazole MICs compared to the parent isolate. Thus, in our hands, the A351V amino acid substitution does not appear to directly affect fluconazole resistance as measure by CLSI broth microdilution assay. However, given the prevalence of ERG3 mutations in resistant clinical isolates and their co-occurrence with ERG11 mutations, the possibility remained that mutations in ERG3 were conferring a compensatory fitness benefit to the cell in the presence of ERG11 mutations that impair native enzyme function.

\section{A homozygous ERG11 ${ }^{\mathrm{Y} 132 \mathrm{H}, \mathrm{K} 143 \mathrm{~T}}$ mutant is conditionally constructed in combination with $E R G 3^{\mathrm{A} 351 \mathrm{~V}}$}

To this end, we aimed to create double mutant strains containing two copies of either the $E R G 3^{\mathrm{WT}}$ allele or the $E R G 3^{\mathrm{A} 351 \mathrm{~V}}$ allele in combination with mutant ERG11 alleles. These mutant ERG11 alleles encoded for select amino acid substitutions previously shown to affect basal catalytic turnover rate of the Erg11 enzyme. The original intent in creating these mutants was to determine whether the strains containing the mutant $E R G 3^{\mathrm{A} 351 \mathrm{~V}}$ allele conferred any additional benefit to fitness over $E R G 3^{\mathrm{WT}}$ when either is combined with activity-altering ERG11 mutations. In the case of the double amino acid substitution Y132H\&K143R in Erg11, we were able to successfully insert both copies of ERG11 in an ERG $3^{\mathrm{A} 351 \mathrm{~V}}$ background, but not in an $E R G 3^{\mathrm{WT}}$ background. Repeated attempts to insert two copies of the ERG11 ${ }^{\mathrm{Y} 132 \mathrm{H} \& \mathrm{~K} 143 \mathrm{R}}$ allele in SC5314 yielded a single copy of the mutant allele, and interestingly, the Y132H unaccompanied by K143R on the second replaced allele, as revealed by Sanger sequencing. Susceptibility testing of the two independently created strains SCERG11R2S2L1 and SCERG11R2S2L2 (ERG1 $1^{\mathrm{Y} 132 \mathrm{H}, \mathrm{K} 143 \mathrm{R}}:: \mathrm{FRT} /$ ERG11 $\left.{ }^{\mathrm{Y} 132 \mathrm{H}}:: \mathrm{FRT}\right)$ had a fluconazole MIC of $1 \mu \mathrm{g} / \mathrm{mL}$, a 4-fold increase in MIC compared to SC5314 (0.25 $\mu \mathrm{g} / \mathrm{mL})$. Comparatively, the strains SCERG3R1S1ERG11R2S2A and SCERG3R1S1ERG11R2S2B, which contained two copies of both $E R G 3^{\mathrm{A} 351 \mathrm{~V}}$ and $E R G 11^{\mathrm{Y} 132 \mathrm{H}, \mathrm{K} 143 \mathrm{R}}$, displayed an 8-fold increase (MIC $\mathrm{FLU}$ : $2 \mu \mathrm{g} / \mathrm{mL}$ ) over SC5314, likely because of the additional K143R substitution present in the second allele.

\section{A homozygous ERG11 ${ }^{\mathrm{G} 307 \mathrm{~S}}$ mutant cannot be constructed}

Surprisingly, we were unable to retain both copies of the $E R G 11^{\mathrm{G} 307 \mathrm{~S}}$ allele in our strains. The single amino acid substitution G307S was unable to be produced in either ERG3 background strain. Repeated attempts were made to construct the above strains, and Southern blotting in conjunction with screening using a nourseothricin drug selection marker seemed to confirm successful integration of allelic replacement cassettes. Despite this, subsequent nucleotide sequencing of tentatively successful strains repeatedly 
Table 4-3. 24-hour fluconazole MICs for $C$. albicans strains in Chapter 4

\begin{tabular}{|c|c|c|}
\hline Strain & $\begin{array}{l}\mathrm{MIC}_{\mathrm{FLU}} \\
\left(\mu \mathrm{g} \mathrm{mL^{-1 }}\right)\end{array}$ & ERG3 genotype \\
\hline SC5314 & 0.25 & WT \\
\hline SCERG3R1S1A & 0.25 & $E R G 3^{A 351 V} \because: F R T / E R G 3^{A 351 V} \because \because F R T$ \\
\hline SCERG3R1S1E & 0.25 & $E R G 3^{A 351 V}: \because F R T / E R G 3^{A 351 V}: \because F R T$ \\
\hline SCERG11R2S2L1 & 1 & $E R G 11^{Y 132 H, K 143 R}: \because F R T / E R G 11^{Y 132 H}: \because F R T$ \\
\hline SCERG11R2S2L2 & 1 & ERG11 ${ }^{Y 132 H, K 143 R} \because: F R T / E R G 11^{Y 132 H} \because: F R T$ \\
\hline SCERG3R1S1ER11GR2S2A & 2 & $\begin{array}{l}E R G 3^{A 351 V}: \because F R T / E R G 3^{A 351 V}: \because F R T \\
E R G 11^{Y 132 H, K 143 R}: \because F R T / E R G 11^{Y 132 H, K 143 R}: \because F R T\end{array}$ \\
\hline SCERG3R1S1ERG11R2S2B & 2 & $\begin{array}{l}E R G 3^{A 351 V}: \because F R T / E R G 3^{A 351 V}: \because F R T \\
E R G 11^{Y 132 H, K 143 R}: \because F R T / E R G 11^{Y 132 H, K 143 R}: \because F R T\end{array}$ \\
\hline SCERG3M4A & $>64$ & $\operatorname{erg} 3 \triangle \because: F R T / \operatorname{erg} 3 \triangle \because: F R T \Delta$ \\
\hline SCERG3M4B & $>64$ & $\operatorname{erg} 3 \triangle \because: F R T / \operatorname{erg} 3 \triangle \because \because F R T \Delta$ \\
\hline
\end{tabular}


revealed retention of only a single copy of either the ERG11 ${ }^{\mathrm{G} 307 \mathrm{~S}}$ mutant allele. The inability to retain both copies of the mutant ERG11 alleles suggests that there may be a selective disadvantage for $C$. albicans to have the G307S amino acid substitution in Erg11 in the absence of drug or other cell stressors.

\section{Discussion}

We have known for some time that mutations in the ERG11 gene can have variable effects on azole resistance. However, the differences observed in Erg11 enzyme function has only recently been discovered. Warrilow et al. measured the relative velocities of purified recombinant $C$. albicans Erg11 protein expressed from E. coli and found that compared to the wild type enzyme, enzymes containing even a single amino acid substitution altered the protein's catalytic turnover significantly (141). From these findings, it was revealed that changes in the amino acid sequence could lower the relative velocity of the Erg11 enzyme to as low as 3\% of the wild type enzyme, and double amino acid substitutions generally resulted in even poorer relative velocities compared to single amino acid substitutions. Importantly, many of these amino acid changes also resulted in higher residual activity in the presence of fluconazole compared to the wild type Erg11.

While the benefit of having such substitutions during azole stress is clear, it raises questions about the potential deleterious effects of Erg11 amino acid changes and fitness costs to the cell in the absence of drug. Amino acid changes, such as the Y132H\&K143R double substitution and the G307S substitution, both of which retain less than $7 \%$ of the catalytic turnover of the wild type Erg11, would not be predicted to be advantageous to a cell in the absence of azole drug stress (141). The low enzyme rate even raises questions whether such an isolate, without compensatory changes elsewhere in the genome, would be viable. Poor Erg11 function as measured by the low catalytic turnover in these cases could have a so-called azole-mimetic effect on the cell, whereby the bottleneck of the ergosterol biosynthesis pathway is exacerbated by innately poor Erg11 function (Figure 4-5). Ergosterol precursors in this case could possibly be shunted to alternative metabolic pathways, producing unwanted byproducts and accumulation of the $14 \alpha$-methylergosta8,24(28)-dien-3 $\beta, 6 \alpha$-diol toxic sterol.

As production of the toxic sterol is dependent on C-5 sterol desaturase activity, it is plausible that changes affecting either the activity of Erg3 or substrate specificity of Erg3 may ameliorate toxic sterol accumulation. Moreover, changes within the genome

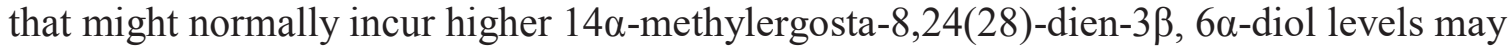
be more permissible when there are accompanying changes in either facet of Erg3 activity. Thus, such Erg3 changes may be the gateway for increased genetic diversity in the ergosterol biosynthetic genes that might otherwise not be possible.

Here, we attempted to determine whether amino acid changes in Erg3, namely the A351 V amino acid substitution, acted as permissive substitutions that allowed for Erg11 variants with slow enzymatic function to persist in $C$. albicans isolates. Substitutions in 


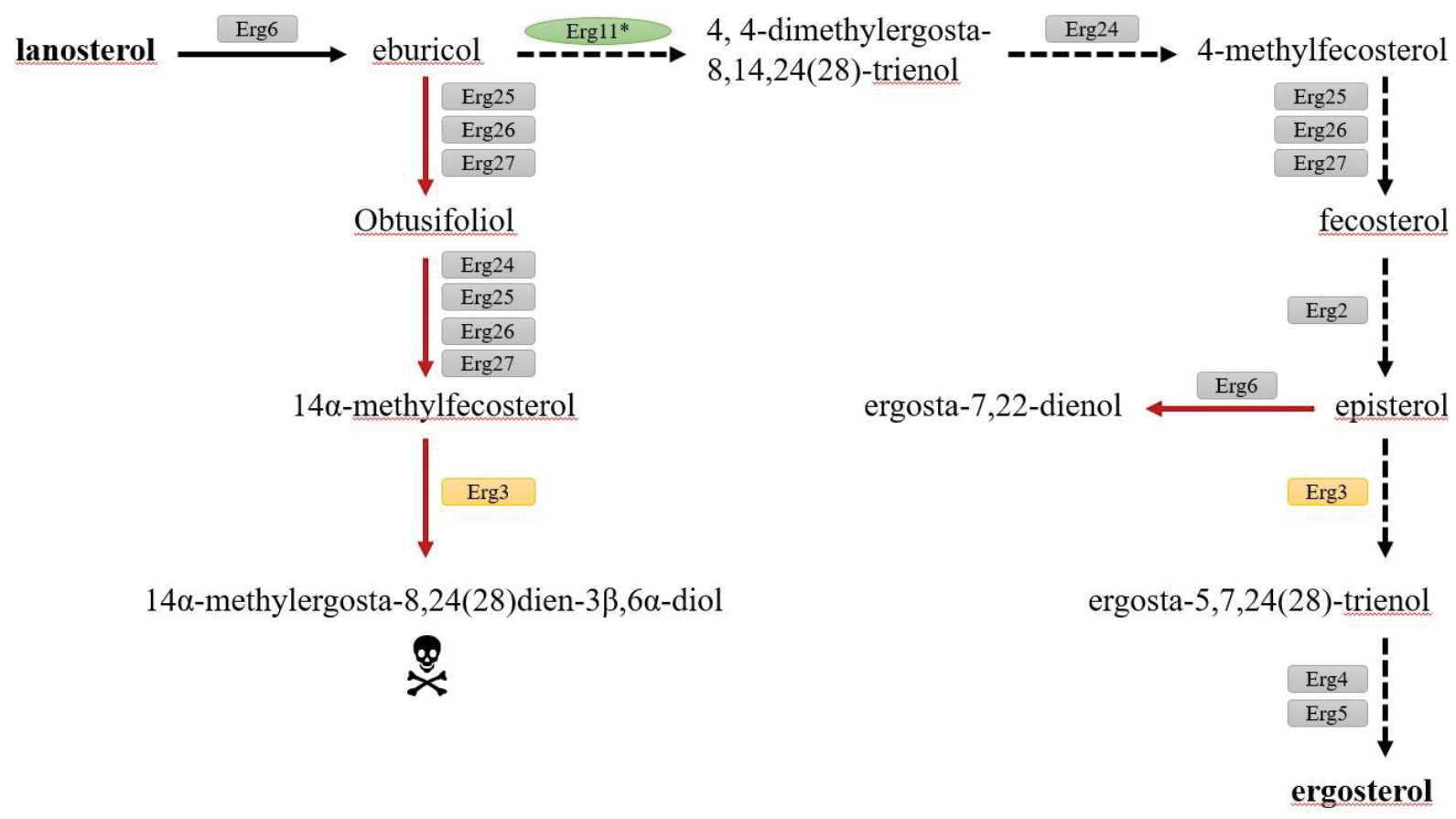

Figure 4-5. Proposed sterol biosynthesis pathway in C. albicans cells with ERG11 mutations resulting in slow Erg11 enzyme function

Simplified representation of proposed consequences to the sterol biosynthesis pathway when Erg11 activity is slowed or lowered as a result of ERG11 mutations. The azole target enzyme Erg11 is shown in green and the mutant Erg11 protein with poor function is labeled with an asterisk. Erg3 is shown in yellow. Red arrows represent alternate biosynthesis pathways. Dashed arrows represent the biosynthetic pathway affected by low Erg11 catalytic turnover. 
Erg3 that might reduce toxic sterol production through alterations of enzymatic activity would be expected to also reduce susceptibility to the azole antifungals directly.

However, no detectable changes in susceptibility were observed in $E R G 3^{\mathrm{A} 351 \mathrm{~V}}$ strains. It is possible that changes in susceptibility were too subtle to be detected through broth microdilution assay or that perhaps lower concentration ranges for fluconazole should be used going forward.

By obtaining whole cell membrane sterol profiles, we hoped to readily identify differences in sterol content between ERG11 mutants with and without ERG3 mutations. Isolates with multiple Erg11 mutations or mutations known to affect enzyme activity might be expected to possess lower percentages of ergosterol and potentially higher levels of $14 \alpha$-methylergosta-8,24(28)-dien-3 $\beta, 6 \alpha$-diol. However, we were unable to identify significant differences between the ergosterol content of mutant and non-mutant $E R G 3$ isolates. One possible explanation for this may be that alterations to total sterol content may be relatively minor. It is also possible that the alterations in sterol content and accumulation of toxic sterols may occur more slowly in these isolates than expected, and that sterol profiles changes would be more apparent after longer periods of growth than what was performed here.

Lastly, we were unable to generate the $E R G 11^{\mathrm{Y} 132 \mathrm{H}, \mathrm{K} 143 \mathrm{R}}$ and $E R G 11^{\mathrm{G} 307 \mathrm{~S}}$ mutants in a background with $E R G 3^{\mathrm{WT}}$. Previous data suggests that both Erg11 variants retain extremely poor function relative to the wild type enzyme (141). By comparing these variant Erg11 proteins with and without the Erg3 A351V amino acid substitution, we hoped to observe differences in fitness based on our selection of Erg11 variants with particularly slow turnover. To our knowledge, the Y132H\&K143R substitutions and the G307S substitution in Erg11 have not been reported in clinical isolates solely by themselves (i.e. without additional, potentially compensatory Erg11 substitutions) and without an accompanying substitution in Erg3. It is possible that mutations resulting in these substitutions are a "double-edged sword" for the cell, trading normal Erg11 enzyme function for azole resistance. Such mutations would only persist in the presence of azole stress and possibly also only with accompanying compensatory mutations.

Our ability to generate the ERG11 $1^{\mathrm{Y} 132 \mathrm{H} \& \mathrm{~K} 143 \mathrm{R}}$ mutant in the ERG $3^{\mathrm{A} 351 \mathrm{~V}}$ strain but not the $E R G 3^{\mathrm{WT}}$ strain raises interesting questions regarding the role of ERG3 mutations in azole resistance. C-5 sterol desaturase-mediated azole resistance has so far only been described to occur as a result of amino acid substitutions in Erg3 that cripple or abolish desaturase activity. Abolition of Erg3 function results in an inability to metabolize the toxic sterol precursor, $14 \alpha$-methylfecosterol to $14 \alpha$-methylergosta-8,24(28)-dien-3 $\beta, 6 \alpha$ diol in the presence of azoles, conferring azole resistance. This is most likely the same mechanism of azole resistance occurring in isolate 60, which had a nonsense mutation in $E R G 3$, preventing transcription and subsequent translation of the full length C-5 sterol desaturase.

However, our findings leave open the possibility that substitutions in Erg3 may also play a peripheral role in azole resistance, enabling C. albicans to increase mutability of ERG11 and possibly other ergosterol biosynthesis genes with mutations that would 
otherwise be unfavorable. If true, mutations in $E R G 3$ could prove to be a valuable predictor of patient response to azole treatment, as ERG3 mutations may indicate the cells proclivity to develop additional mutations in the azole-resistance gene ERG11. While this remains a possibility, further investigation must be conducted to determine the role of the ERG3 mutations observed in clinical isolates of C. albicans.

\section{Materials and Methods}

\section{Strains and growth conditions}

The C. albicans strains used in this study are listed in Table 4-3. Cells were streaked on the YPD agar plates from frozen $40 \%$ glycerol stocks and grown overnight at $30^{\circ} \mathrm{C}$. Colonies from YPD agar plates were grown overnight in YPD liquid media (1\% yeast extract, $2 \%$ peptone, $2 \%$ dextrose) at $30^{\circ} \mathrm{C}$ for all routine genomic DNA extraction. Luria-Bertani (LB) liquid media and agar plates, both containing $100 \mu \mathrm{g} / \mathrm{mL}$ ampicillin or $50 \mu \mathrm{g} / \mathrm{mL}$ kanamycin, were used to grow $E$. coli cells used in plasmid construction and propagation.

\section{Plasmid construction}

Table 4-4 shows the primers used in this study. To construct plasmids containing our transformation cassettes for gene ERG3 and ERG11 gene knockout or replacement, we utilized the pSFS2-derived plasmid pBSS2 containing the SAT1 nourseothricin resistance marker and FLP recombinase, described previously (202). To generate the ERG3 deletion cassette, flanking sequences of the 5' and 3' ends of the ERG3 openreading frame (ORF) was amplified from SC5314 genomic DNA using primers CaERG3_AF_ApaI and CaERG3_BR_XhoI or CaERG3_CF_SacII and CaERG3_DR_SacI, respectively. Fragments were digested and ligated to plasmid pBSS2 to create plasmid pBSS2-ERG3ABCD. The replacement cassette used in putting two copies of the $E R G 3^{\mathrm{A} 351 \mathrm{~V}}$ allele back into $C$. albicans was constructed by amplifying the ERG3 ORF using primers CaERG3_AF_ApaI and CaERG3_ER_XhoI. The resulting fragment and plasmid pBSS2-ERG3 $\overline{A B C D}$ were digested with restriction enzymes ApaI and XhoI and ligated together to form pBSS2-ERG3AECD.

To construct the mutant $E R G 11^{\mathrm{Y} 132 \mathrm{H}, \mathrm{K} 143 \mathrm{R}}$ allele, primer sets CaERG11-1F \& CaERG11SOE-3R Y132H and CaERG11SOE-2F Y132H \& CaERG11-4R were used to

amplify fragments of the ERG11 gene from isolate 13 containing the ERG1 $1^{\mathrm{K} 143 \mathrm{R}}$ allele previously characterized (139). For the $E R G 11^{\mathrm{G} 307 \mathrm{~S}}$ allele, primer sets CaERG11-1F \& CaERG11SOE-6R and CaERG11SOE-5F \& CaERG11-4R amplified ERG11 gene fragments from isolate 26 genomic DNA. Each respective set fragments was combined and amplified using short-overlapping extension PCR utilizing primers CaERG11-AF (ApaI) and CaERG11-BR (XhoI) to make a full length ERG11 allele containing either the Y132H\&K143R or G307S amino acid substitutions. Fused PCR products were digested 
Table 4-4. Primers used in Chapter 4

\begin{tabular}{|c|c|}
\hline Primer & Oligonucleotide sequence $^{\mathrm{a}}$ \\
\hline \multicolumn{2}{|l|}{ Amplification } \\
\hline CaERG3_F_HindIII & 5' - TTGTTAAGCTTTTTTTTCTTCCACCTATTTTG - 3' \\
\hline CaERG3_R_BAMHI & $5^{\prime}$ - CACACGGATCCATATAACCCTTGATTCC - ${ }^{\prime}$ \\
\hline CaERG1̄̄-ĀF (ApaI) & 5' - GGGCCCGGGTTATTTGAGAACAGCC - 3, \\
\hline CaERG11-BR (XhoI) & 5' - CTCGAGCCAGTGGACAAAAACCATCA - 3' \\
\hline \multicolumn{2}{|l|}{ Short, overlapping extension } \\
\hline CaERG11-1F & 5' - CAATCTATACGACGACATTTAACTTTTT - ${ }^{\prime}$ ' \\
\hline CaERG11SOE-3R Y132H & 5' - GACAATCATGAATAACCCCTTTACCGAAAA - 3' \\
\hline CaERG11SOE-2F Y132H & 5' - GGGGTTATTCATGATTGTCCAAATTCCAGA - 3' \\
\hline CaERG11SOE-6R & 5' - АCСТTTTTCTTTCAATAATTCAACAACT - 3' \\
\hline CaERG11SOE-5F & 5' - ATTATTGAAAGAAAAAGGTGGTGATTTG - 3' \\
\hline CaERG11-4R & 5' - GCTGTTGTTGTTGTTGAAAGAAA - 3' \\
\hline \multicolumn{2}{|l|}{ Sequencing } \\
\hline CaERG3SeqA & $5^{\prime}$ - GACCTAAGATTCCTACAATC - $3^{\prime}$ \\
\hline CaERG3SeqB & 5' - CAGCTACTGATTTCATTAATAC - 3' \\
\hline CaERG3SeqC & 5' - GGTTCTTCCAATCTTTACC - 3' \\
\hline CaERG3SeqD & $5^{\prime}$ - GGAAAAATAGTCAATGGTCC - 3' \\
\hline CaERG3SeqE & 5' - GTAAACAGATGGCCAGTG - 3' \\
\hline CaERG3SeqF & 5' - TCTGAAAATGTTTGATCTGGC - 3' \\
\hline CaERG11SeqA & 5' - GCCACCACACCCTATGGCTATT - 3' \\
\hline CaERG11SeqB & 5' - TATTTTCACTGCTTCAAGATCT - $3^{\prime}$ \\
\hline CaERG11SeqC & 5' - CCAAAAGGTCATTATGTTTTAG - 3' \\
\hline CaERG11SeqD & 5' - CATACAAGTTTCTCTTTTTTCC - 3 \\
\hline CaERG11SeqE & $5^{\prime}$ - CATTTAGGTGAAAAACCTCATT - 3' \\
\hline CaERG11SeqF & 5' - TACTCCAGTTTTCGGTAAAGGG - 3' \\
\hline \multicolumn{2}{|l|}{ Deletion and Replacement } \\
\hline CaERG3_AF_ApaI & 5' - ATCTGATTTATATATGGGCCCCAAGTGTTTG - 3' \\
\hline CaERG3_BR_XhoI & 5' - AAAAGATAATAGTCTCGAGTTTCTAGTACG - \\
\hline CaERG3_CF_SacII & 5' - ATAGCCGCGGTAACTCTTACAGAAGACC - 3' \\
\hline CaERG3_DR_SacI & 5' - TGTGATGTGAGCTCGTTAGTATTATTTTCA - 3' \\
\hline CaERG3_ER_XhoI & 5' - TGTGATGTACTCGAGTTAGTATTATTTTCA - 3' \\
\hline
\end{tabular}

${ }^{a}$ Underlined bases indicate a restriction enzyme cutting sequence, red bases indicate a base change from reference sequence for base specific mutagenesis 
with restriction enzymes ApaI and XhoI and ligated into pBSS2, a derivative of pSFS2 containing the CaSAT1/FLP cassette in a pBluescript backbone (217). The resulting ligation yielded plasmid pERG11R2 and pERG11R4, containing the ERG11 ${ }^{\mathrm{Y} 132 \mathrm{H}, \mathrm{K} 143 \mathrm{R}}$ and $E R G 11^{\mathrm{G} 307 \mathrm{~S}}$ allele, respectively.

\section{$E R G 3$ and $E R G 11$ amplification and sequencing}

For sequencing of ERG3 in our C. albicans clinical isolate collection, the ORF for ERG3 and ERG11 was amplified using primers CaERG3_F_HindIII \& CaERG3_R_BAMHI and CaERG11-AF (ApaI) \& CaERG11-BR (XhoI), respectively. The QIAquick® PCR Purification Kit (Qiagen) was used to purify PCR products. Approximately $100-150 \mathrm{ng}$ of purified PCR product containing the ERG3 ORF was used in sample preparation with the ERG3 sequencing primers CaERG3SeqA, CaERG3SeqB, CaERG3SeqC, CaERG3SeqD, CaERG3SeqE, and CaERG3SeqF for Sanger sequencing by capillary electrophoresis. For ERG11, the sequencing primers used were CaERG11SeqA, CaERG11SeqB, CaERG11SeqC, CaERG11SeqD, CaERG11SeqE, and CaERG11SeqF. An ACI 3130XL genetic analyzer was used in processing sequence reads and sequencing was accomplished in duplicate in independently grown isolates.

\section{C. albicans strain construction}

A DNA fragment containing the $S A T 1 / \mathrm{FLP}$ cassette flanked by sequences at the 5' and 3' end of the ERG3 gene was excised by ApaI and XhoI restriction enzymes. The resulting fragment was transformed via electroporation into C. albicans isolate SC5314, disrupting a single copy of ERG3 in successfully transformed cells. Cells were screened on YPD-agar plates containing $(200 \mu \mathrm{g} / \mathrm{mL})$ nourseothricin and successful cells were grown in YPD for 24 hours to recycle the $S A T 1 /$ FLP marker. A repeat transformation and screening was performed to generate the $\operatorname{erg} 3 \Delta / \Delta$ strain SCERG3M4A. For strains containing the $E R G 3^{\mathrm{A} 351 \mathrm{~V}}$ mutant allele, the replacement cassette was excised out of plasmid pBSS2-ERG3AECD with ApaI and XhoI and transformed via electroporation into SCERG3M4A. As above, recycling of the nourseothricin marker, repeat transformation and screening on YPD-agar with nourseothricin ensured both alleles contained the replacement cassette, generating strains SCERG3R1S1A1, SCERG3R1S1A2, SCERG3R1S1E1, and SCERG3R1S1E2. To generate strains possessing both mutant ERG11 and ERG3 alleles, we transformed the ERG11 replacement cassettes from pERG11R2 and pERG11R4 into SCERG3R1S1A or SC5314. Two sequential integrations of the replacement cassette of pERG11R2 generated homozygous replacement of the native ERG11 alleles with two copies of ERG11 $1^{\mathrm{Y} 132 \mathrm{H} \& \mathrm{~K} 143 \mathrm{R}}$. Southern blotting visually confirmed homozygous allelic deletion and/or replacement of all strains using methods previously described (141). Confirmation of allelic sequences were confirmed by Sanger sequencing of either the ERG3 or ERG11 ORF. 


\section{Azole susceptibility testing}

MICs for fluconazole were determined by broth microdilution assay in accordance with the Clinical Laboratory and Standards Institute $(200,201)$. To reduced trailing growth observed in isolates, a slight modification through addition of $2 \%$ glucose to RPMI media was made. Assay was prepared in 96-well round bottomed plates and incubated for 24 hours at $35^{\circ} \mathrm{C}$. Measurements were visually read after 24 hours. MIC was defined as the concentration at which approximately $50 \%$ growth inhibition was seen compared to isolate or strain growth in drug-free control wells. 


\section{CHAPTER 5. IN VITRO ACTIVITIES OF THE NOVEL INVESTIGATIONAL TETRAZOLE VT-1161 AND VT-1598 COMPARED TO THE TRIAZOLE ANTIFUNGALS AGAINST AZOLE-RESISTANCE STRAINS AND CLINICAL ISOLATES OF CANDIDA ALBICANS ${ }^{3}$}

\section{Introduction}

Candida albicans is a dimorphic yeast and opportunistic pathogen that is known to cause a wide range of infections in healthy and immunocompromised patients. In the United States, C. albicans is the leading Candida species identified in oropharyngeal and vulvovaginal infections, where recurrent infections remain problematic $(16,69,185,211$, 218). In more serious systemic disease such as bloodstream infections (BSI), Candida species collectively are the fourth-leading cause of nosocomial BSI in the United States (12). Moreover, resistance to currently available antifungal agents continues to be a problem, particularly given the relatively limited armamentarium against fungal infections $(32,219-222)$. In particular, azole antifungal resistance in Candida spp. threatens to diminish the efficacy of arguably the most widely used antifungal drug class (223). Appropriate clinical use of available drugs on the market and eventual expansion of the antifungal arsenal is therefore paramount to safeguarding its effectiveness.

Azole antifungal resistance in $C$. albicans can be attributed to multiple mechanisms. First, efflux pump overexpression, such as the ATP-binding cassette (ABC) transporters Cdr1 and Cdr2 as well as the major facilitator transporter Mdr1, prevents drug accumulation within the yeast cell $(83,84,104,196)$. Second, increased production of the azole target 14 $\alpha$-lanosterol demethylase (CYP51) can attenuate the inhibitory effects of the azoles drug class $(106,108,115)$. Increases in efflux pump and drug target production is often the result of gain-of-function mutations in zinc cluster transcription factors (ZCFs) (Tac1 for CDR1 and CDR2, Mrr1 for MDR1, Upc2 for ERG11) that regulate their gene expression, though polyploidy of chromosomes in the yeast genome can also result in increased expression of the genes encoding these azole-resistance determinants. Third, mutations in ERG11 can confer azole resistance through alteration of the drug target $(122-124,139)$. Lastly, alternative sterol biosynthesis as a result of changes within the ergosterol biosynthetic pathway allows some $C$. albicans isolates to circumvent the effects of azole inhibition altogether $(72,142,145,224)$.

VT-1161 and VT-1598 are novel tetrazole antifungal agents with high specificity for fungal CYP51 compared to human CYP enzymes $(188,189,225)$, and thus may have improved adverse effect and drug-drug interaction profiles due to lesser off-target inhibition. In this study, we compare the in vitro activity of the novel tetrazoles

\footnotetext{
${ }^{3}$ Reprinted from final submission with permission. Nishimoto AT, Wiederhod NP, Flowers SA, Zhang Q, Kelly SL, Morschhäuser J, Yates CM, Hoekstra WJ, Schotzinger RJ, Garvey EP, Rogers PD. (2019) In vitro activities of the novel investigational tetrazoles VT-1161 and VT-1598 compared to the triazole antifungals against azole-resistant strains and clinical isolates of Candida albicans. AntiMicrob Agents Chemother. doi:10.1128/aac.00341-19:AAC.00341-19.
} 
VT-1161 and VT-1598 to the current triazole antifungals fluconazole, voriconazole, itraconazole, and posaconazole against a collection of clinical isolates and laboratory strains with known resistance mechanisms.

\section{Results}

\section{In vitro activity of VT-1161 and VT-1598 against fluconazole-susceptible and fluconazole-resistant clinical isolates}

VT-1161 and VT-1598 showed potent in vitro activity against 68 previously described clinical isolates of $C$. albicans, the majority (57 of 68) of which were fluconazole-resistant (MIC $\geq 8 \mu \mathrm{g} / \mathrm{mL}$ ) and possessed multiple known azole resistance mechanisms (Appendix B, Table A-1 to B-3) (115). Both VT-1161 and VT-1598 had lower $\mathrm{MIC}_{50}$ values $(0.06$ and $0.125 \mu \mathrm{g} / \mathrm{mL}$, respectively), and VT-1598 had a lower $\mathrm{MIC}_{90}$ value $(0.25 \mu \mathrm{g} / \mathrm{mL})$ when compared to the other tested azole antifungals (Table 5-1). VT-1161 and VT-1598 MICs were $\leq 0.015 \mu \mathrm{g} / \mathrm{mL}$ against the 11 fluconazolesusceptible isolates within the collection, and the VT-1598 MICs were $0.03 \mu \mathrm{g} / \mathrm{mL}$ against 33\% (19 of 57) of the fluconazole-resistant clinical isolates. This suggests that some fluconazole-resistance mechanisms do not affect the in vitro potency of VT-1598. Posaconazole also demonstrated activity against many, but not all, of the same fluconazole-resistant isolates, as posaconazole MICs were within a two-fold increase (1dilution difference) to those of the fluconazole-susceptible isolates for 15 of the fluconazole-resistant isolates. Using this same metric, VT-1161 maintained in vitro potency against 8 fluconazole-resistant clinical isolates, which was comparable to that of voriconazole (6 isolates) and greater than that of itraconazole ( 2 isolates). Overall, VT1598 and VT-1161 thus appear to have additional activity against several fluconazoleresistant isolates, and in this respect are at least comparable to commercially available triazoles.

VT-1598 MICs were elevated at least four-fold $(\geq 0.06 \mu \mathrm{g} / \mathrm{mL}$, range 0.06 to $>8$ $\mu \mathrm{g} / \mathrm{mL}$ ) against 38 fluconazole-resistant isolates compared to its activity against the fluconazole-susceptible isolates. VT-1161 MICs were elevated at least four-fold $(\geq 0.06$ $\mu \mathrm{g} / \mathrm{mL}$, range 0.06 to $>8 \mu \mathrm{g} / \mathrm{mL}$ ) against 49 fluconazole-resistant isolates. Five clinical isolates displayed highly elevated VT-1598 and VT-1161 MICs (range 4 to $>8 \mu \mathrm{g} / \mathrm{mL}$ ) and also high fluconazole, voriconazole, itraconazole, and posaconazole MICs. Sequencing and/or relative quantitation of mRNA expression of known resistance genes revealed that four of these isolates overexpressed $C D R 1$ relative to the $C D R 1 \mathrm{mRNA}$ levels of fluconazole-susceptible clinical isolates (115). The fifth isolate contained a premature stop codon in ERG3, resulting in truncation of the protein after Gly130, which likely explains its significantly elevated resistance not only to VT-1161 and VT-1598, but also to all other tested azole antifungals.

To gain additional insight on the determinants that could confer decreased

susceptibility to VT-1161 and VT-1598 in the clinical isolates, a point-biserial correlation 
Table 5-1. Geometric mean $\mathrm{MICs}, \mathrm{MIC}_{50}, \mathrm{MIC}_{90}$, and ranges $(\mu \mathrm{g} / \mathrm{mL})$ for tested compounds against 68 clinical isolates of $C$. albicans

\begin{tabular}{lcccc}
\hline Drug & GM MIC & MIC $_{\mathbf{5 0}}$ & MIC $_{\mathbf{9 0}}$ & Range \\
\hline VT-1161 & 0.15 & 0.125 & 1 & $\leq 0.015->8$ \\
VT-1598 & 0.05 & 0.06 & 0.25 & $\leq 0.015->8$ \\
Fluconazole & 20.2 & 32 & $>64$ & $\leq 0.125->64$ \\
Voriconazole $^{1}$ & 0.32 & 0.5 & 2 & $\leq 0.03->16$ \\
Itraconazole $^{1}$ & 0.31 & 0.25 & 1 & $\leq 0.03->16$ \\
Posaconazole $^{1}$ & 0.2 & 0.5 & 2 & $\leq 0.03->16$ \\
\hline
\end{tabular}

${ }^{1} \mathrm{n}=66$ clinical isolates for itraconazole and posaconazole 
between the $\log _{2}$-fold increase in VT-1598 and VT-1161 MICs and the mRNA expression levels of $C D R 1, M D R 1$, and $E R G 11$ in the clinical isolates was performed.

The $\log _{2}$-fold increase in MICs was compared to the baseline MIC measurement for VT1598 and VT-1161 against fluconazole-susceptible isolates (MIC $\leq 0.015)$ and expression levels of either CDR1, MDR1, and ERG11 were measured via RT-qPCR in a previous study (115). The majority of fluconazole-resistant clinical isolates exhibited increased $C D R 1$ expression, however there was no significant correlation between $C D R 1$ expression and VT-1598 resistance $(\mathrm{p}=0.287)$. In contrast, higher levels of CDR 1 expression did positively correlate with increasing VT-1161 MICs $(p<0.01)$. Similarly, while there was no relationship between MDR1 expression and VT-1598 MIC ( $\mathrm{p}=$ $0.105)$, there was a slight positive correlation between $M D R 1$ expression and increased VT-1161 MIC ( $<$ 0.05). No significant correlation was established with either drug and ERG11 expression ( $\mathrm{p}=0.512$ and $\mathrm{p}=0.355$ for VT-1598 and VT-1161, respectively).

VT-1598 and VT-1161 MICs against the clinical isolates were plotted directly against those of fluconazole, voriconazole, itraconazole, and posaconazole to visualize relative susceptibility differences (Figure 5-1). As previously noted, both VT-1161 and VT-1598 retained potency against several fluconazole-resistant isolates, and all isolates with reduced VT-1598 or VT-1161 potency were resistant to fluconazole. By comparison, VT-1161 MICs were disproportionately higher against some clinical isolates compared to those of the other tested azoles. One voriconazole-susceptible isolate $(0.125$ $\mu \mathrm{g} / \mathrm{mL}$ ) that contained a K143R ERG11 mutation and exhibited increased CDR 1 expression had an 8-fold increase in the MIC of VT-1161 compared to that observed against fluconazole-susceptible isolates $(\leq 0.015 \mu \mathrm{g} / \mathrm{mL})$. In addition, a single itraconazole-susceptible isolate $(0.125 \mu \mathrm{g} / \mathrm{mL})$ demonstrated a 32-fold increase in the VT-1161 MIC $(0.5 \mu \mathrm{g} / \mathrm{mL})$. This isolate contained three Erg11 amino acid substitutions (F126L, Y132F, H283R), but lacked other obvious azole resistance mechanisms. Against isolates with low posaconazole MICs (range $\leq 0.03$ to $0.25 \mu \mathrm{g} / \mathrm{mL}$ ), seven displayed $\geq 16$ fold increases in VT-1161 MIC over fluconazole-susceptible isolates. Among these seven isolates, all contained various $E R G 11$ mutations, four overexpressed CDR 1 by at least two-fold, one overexpressed both $E R G 11$ and $C D R 1$, and one overexpressed MDR 1 . In contrast, there were no strong outliers for VT-1598 MICs when compared against those of the triazoles.

\section{In vitro activity of VT-1161 and VT-1598 against strains with known azole resistance mechanisms}

To identify determinants of VT-1161 and VT-1598 resistance, we evaluated the influence of specific azole-resistance mechanisms on VT-1161 and VT-1598 MICs when placed in the fluconazole-susceptible isolate SC5314 (Figure 5-2). Increased CDRI and $C D R 2$ expression through artificial activation of the TAC1 gene increased the VT-1161 MICs more than eight-fold compared to the susceptible parent strain. This increase in VT-1161 MIC was diminished, but not completely abolished, when the CDR1 gene was deleted, suggesting that overexpression of $C D R 1$ as well as other Tacl target genes (most 
Figure 5-1. Comparison of the MICs of VT-1161 and VT-1598 against the MICs of triazoles

VT-1161 and VT-1598 MICs versus the of MICs of (A) fluconazole, (B) voriconazole, (C) itraconazole, and (D) posaconazole in a collection of C. albicans clinical isolates.

Plotted points represent the MICs of clinical isolates, with darker points representative of multiple, superimposed points. Concentration of points to the lower right of each plot represent favorable activity (low MICs relative to susceptible isolates) for VT-1161 or VT-1598 versus the comparator azole. Conversely, points concentrated to the top left of each plot represent isolates with high MICs of VT-1161 and VT-1598 relative to the comparator azole. 
a

MICs of VT-1161 versus fluconazole in clinical C. albicans isolates

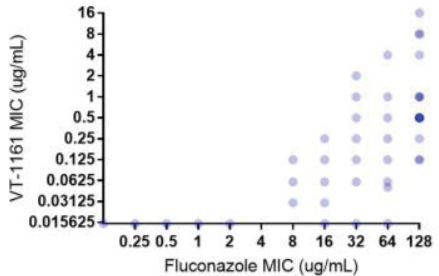

b

MICs of VT-1161 versus voriconazole in clinical C. albicans isolates

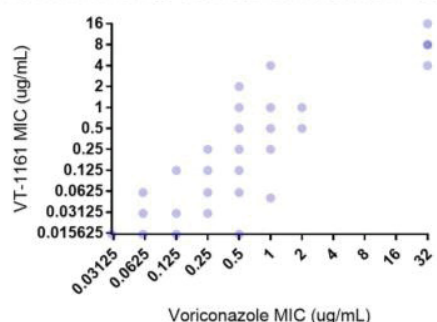

C

MICs of VT-1161 versus itraconazole in clinical C. albicans isolates

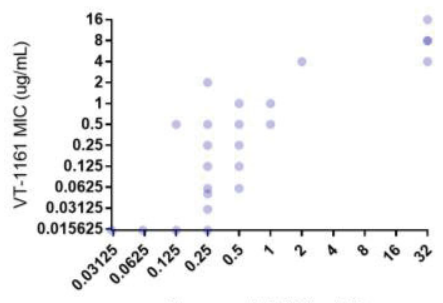

Itraconazole MIC (ug/mL)

d

MICs of VT-1161 versus posaconazole in clinical C. albicans isolates

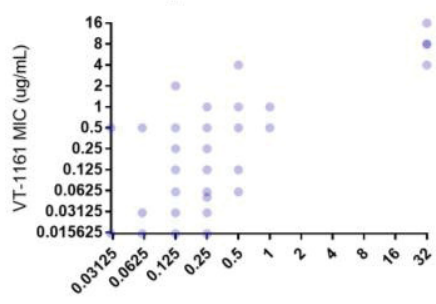

Posaconazole MIC (ug/mL)
MICs of VT-1598 versus fluconazole in clinical C. albicans isolates

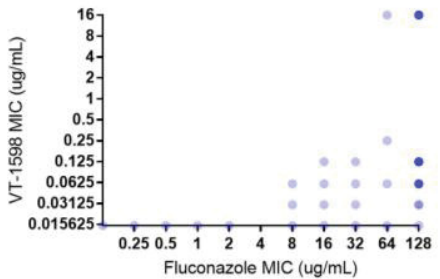

Fluconazole MIC (ug/mL)

MICs of VT-1598 versus voriconazole in clinical C. albicans isolates

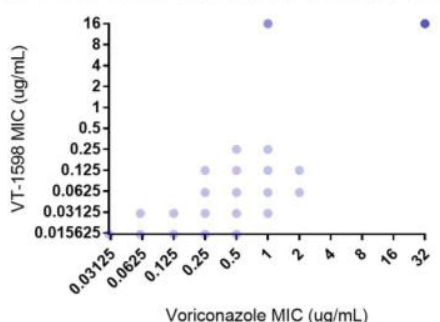

MICs of VT-1598 versus itraconazole in clinical C, albicans isolates

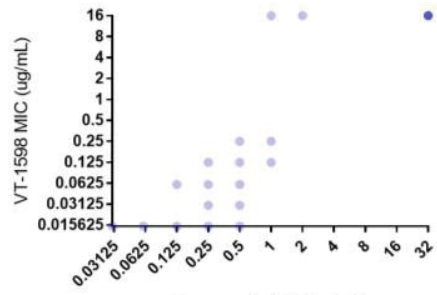

Itraconazole MIC (ug/mL)

MICs of VT-1598 versus posaconazole in clinical C. albicans isolates

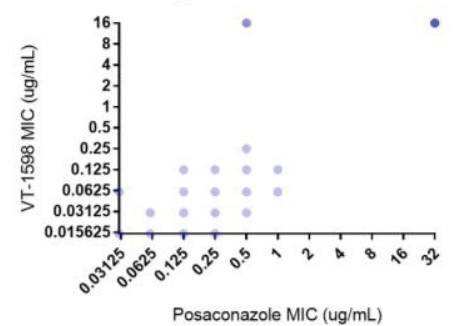


Figure 5-2. MICs of tested azole compounds against strains with individual known azole resistance mechanisms

Tested strains include those containing the artificially-activated transcription factors Tac1 and Mrr1 in strains SCTAC1GAD1A and -B and SCMRR1GAD1A and -B, respectively, as well as $\Delta c d r 1$ derivatives of SCTAC1GAD1A (SC $\Delta c d r 1 \mathrm{TAC} 1 \mathrm{GAD} 1 \mathrm{~A}$ and -B), $\Delta m d r 1$ derivatives of SCMRR1GAD1A and -B (SC $\Delta m d r 1 \mathrm{MRR} 1 \mathrm{GAD} 1 \mathrm{~A}$ and -B), and SCUPC2R14A and -B containing the G648D gain-of-function mutation in UPC2. The MICs for the strains with artificially activated Tac1, Mrr1, and for the $U P C 2^{\mathrm{G} 648 \mathrm{D}}$ gainof-function mutation are displayed as the highest MIC value of both independently created A- and B- strains for each respective transcription factor. The relative foldchange in expression compared to the parent strain SC5314 of (A) CDR1 for SCTAC1GAD1A and B and (B) MDR1 for SCMRR1GAD1A and -B is shown on the left of the figure. The antifungal MICs of the $U P C 2^{\mathrm{G} 648 \mathrm{D}}$ homozygous strains SCUPC2R14A and $-\mathrm{B}$ is shown in $(\mathrm{C})$. 
A

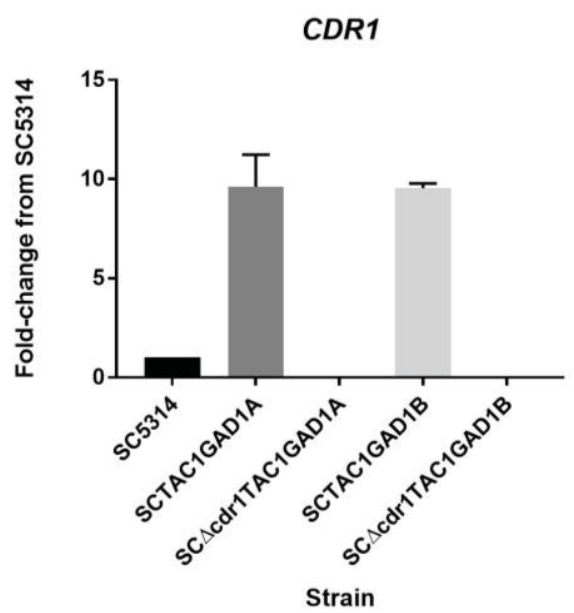

B

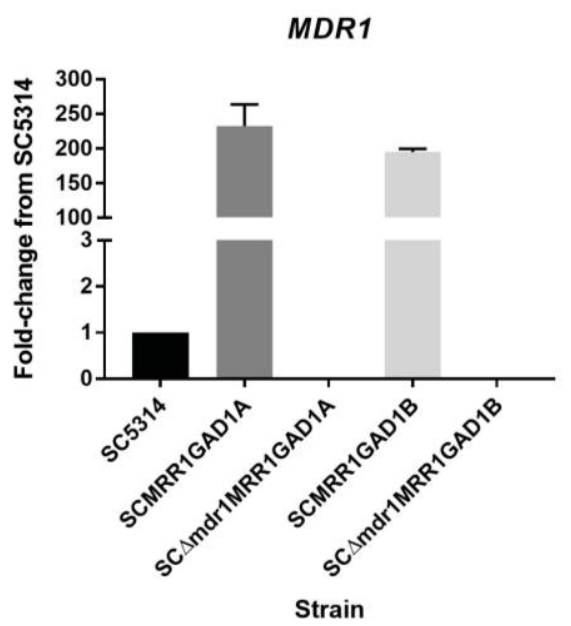

\section{C}

\begin{tabular}{|ccc|}
\multicolumn{3}{c}{ MICs $(\mu \mathrm{g} / \mathrm{mL})$} \\
\hline & $\underline{\text { SC5314 }}$ & $\underline{\text { SCUPC2R14 }}$ \\
$\underline{\text { VT-1598 }}$ & $<0.015$ & $<0.015$ \\
$\underline{\text { VT-1161 }}$ & $<0.015$ & $<0.015$ \\
$\underline{\text { Fuconazole }}$ & 0.25 & 1 \\
$\underline{\text { Voriconazole }}$ & $<0.03$ & $<0.03$ \\
$\underline{\text { traconazole }}$ & 0.125 & 0.125 \\
$\underline{\text { Pos aconazole }}$ & $<0.03$ & $<0.03$ \\
\hline
\end{tabular}

MICs $(\mu \mathrm{g} / \mathrm{mL})$

\begin{tabular}{|cccc|}
\hline & $\underline{\text { SC5314 }}$ & $\underline{\text { SCTAC1GAD1 }}$ & $\underline{\underline{\text { SCACdr1 }}}$ \\
$\underline{\text { VT-1598 }}$ & $\leq 0.015$ & $\leq 0.015$ & $\leq 0.015$ \\
$\underline{\text { VT-1161 }}$ & $\leq 0.015$ & 0.125 & 0.06 \\
$\underline{\text { Fluconazole }}$ & 0.5 & 8 & 2 \\
$\underline{\underline{\text { Voriconazole }}}$ & $\leq 0.03$ & 0.125 & $\leq 0.03$ \\
$\underline{\text { Itraconazole }}$ & 0.125 & 0.25 & 0.125 \\
$\underline{\text { Posaconazole }}$ & $\leq 0.03$ & 0.06 & 0.06 \\
\hline
\end{tabular}

MICs $(\mu \mathrm{g} / \mathrm{mL})$

\begin{tabular}{|cccc|}
\hline & $\underline{\text { SC5314 }}$ & $\underline{\text { SCMRR1GAD1 }}$ & $\underline{\underline{\text { SCAmdr1 }}}$ \\
$\frac{\text { VTR1GAD1 }}{\text { VT-1161 }}$ & $\leq 0.015$ & $\leq 0.015$ & $\leq 0.015$ \\
$\frac{\text { VT-015 }}{\underline{\text { Fuconazole }}}$ & 0.5 & 0.06 & $\leq 0.015$ \\
$\underline{\text { Voriconazole }}$ & $\leq 0.03$ & 16 & 8 \\
$\underline{\underline{\text { Itraconazole }}}$ & 0.125 & 0.125 & 0.06 \\
$\underline{\text { Posaconazole }}$ & $\leq 0.03$ & 0.25 & 0.125 \\
\hline
\end{tabular}


likely $C D R 2$ ) was responsible for the decreased susceptibility to VT-1161. On the other hand, Tac1 activation did not result in reduced susceptibility to VT-1598, as opposed to its effect on VT-1161, fluconazole and voriconazole resistance, which increased between four- and 16-fold compared to that of the parental strain SC5314. Posaconazole and itraconazole MICs were only slightly elevated (two-fold) by the hyperactive Tac1. Thus, it appears that while drug efflux via Cdr1 plays a role in VT-1161 resistance, Tac1 activation and the approximately 10 -fold increase in $C D R 1$ expression are not sufficient to alter MICs of VT-1598.

While a hyperactive Mrr1 did not result in increased resistance to VT-1598, it caused a four-fold increase in the MIC of VT-1161. This increase was abolished upon $M D R 1$ deletion, suggesting that the Mdr1 transporter is involved in VT-1161 resistance. Fluconazole and voriconazole were the only tested azole drugs against the MDRIoverexpressing strain that showed a greater than two-fold increase in MIC (32-fold and four-fold, respectively) over SC5314. By comparison, itraconazole showed a minimal two-fold increase (1-dilution difference) in MIC, and posaconazole MICs were not affected by MDR1 overexpression. Strangely, there was a 4 -fold increase in posaconazole MIC when MDR1 was deleted in the hyperactive Mrr1 strain. However, this is consistent with variability observed for posaconazole MICs in other published strains and fluconazole-susceptible clinical isolates (94).

Upregulated expression of $E R G 11$ via artificial activation of the Upc2 transcription factor also did not affect VT-1161 or VT-1598 MICs. However, despite an approximate 4- to 8-fold increase in ERG11 expression (data not shown), the activated $\mathrm{Upc} 2$ strain failed to demonstrate a relevant change in voriconazole, posaconazole, and itraconazole MICs. Surprisingly, this strain also exhibited no change in fluconazole MIC as has previously been reported. We therefore decided to also test azole susceptibilities in a strain homozygous for the G648D amino acid substitution, previously shown to be the strongest clinically-derived gain-of-function mutation in Upc2 $(113,115)$. Strains SCUPC2R14A and -B overexpressed ERG11 relative to the parent strain SC5314 by 6.4fold (previously published) and 4.5-fold (unpublished data), respectively (data not shown) (115). A modest 2-fold increase in fluconazole MIC in these two strains was observed compared to SC5314, whereas no changes were observed for the MICs of any of the other antifungal agents.

To compare the effects of different alterations in the azole target enzyme on the susceptibility of $C$. albicans to VT-1161 and VT-1598, twelve single Erg11 amino acid substitutions and four double substitutions in Erg11 were tested (Table B-2, Figure 5-3). The Y132F single substitution caused a substantial (16-fold) increase inVT-1161 MIC. Surprisingly, the double amino acid substitutions Y132F\&K143R and Y132F\&F145L had a lesser impact on VT-1161 MIC (eight-fold and four-fold increase, respectively) than the single Y132F substitution alone. Other amino acid substitutions did not have an appreciable effect on VT-1161 MICs, and none of the tested ERG11 mutants showed greater than a two-fold increase in the MIC of VT-1598. The F145L and S405F single mutants and the double substitution mutants D278N\&G464S and 


\section{Fold-change in MIC for ERG11 mutations}

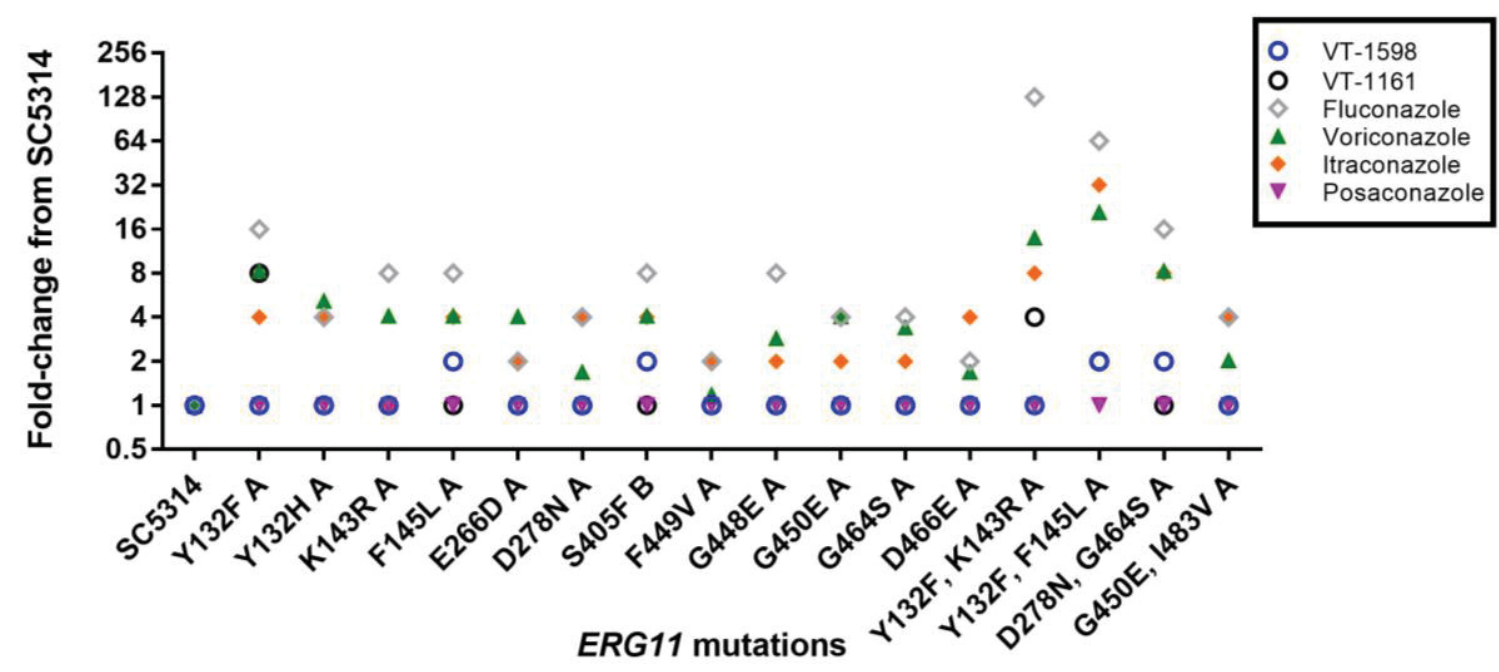

Figure 5-3. Relative fold-change compared to SC5314 in MIC of various azole antifungal agents against strains containing single and double ERG11 mutations Open blue circles represent VT-1598 MICs, while open black circles represent VT-1161. Open grey diamonds represent fluconazole. Solid green triangles represent voriconazole. Solid orange diamonds represents itraconazole, and solid inverted purple triangles represent posaconazole. 
Y132F\&F145L showed a slight two-fold increase in the VT-1598 MIC compared to that against SC5314.

\section{Discussion}

VT-1598 has previously demonstrated a broad spectrum of activity in vitro against yeasts such as Candida and Cryptococcus spp., moulds including Aspergillus spp. and endemic fungi (226), and has shown improved survival and reduced fungal burden in murine models of CNS coccidioidomycosis (227) and cryptococcosis (228). Pertinent to the present study, VT-1598 has also recently shown potent in vitro and in vivo antifungal activity against fluconazole-sensitive and -resistant Candida spp. isolated from chronic mucocutaneous candidiasis patients (187). Structurally, while the tetrazole moiety has lower affinity for interaction with the heme iron of CYP51, other structural modifications have made the drug more fungal-specific. For example, a critical H-bond between VT1598 and the CYP51 enzyme of many fungi likely confers its broad activity (229). This greater selectivity may decrease the potential for undesirable adverse effects and drug interactions that occur with the triazoles through inhibition of human cytochrome P-450 enzymes.

Our study supports the previous finding that VT-1598 has potent activity against C. albicans isolates. Overall, VT-1598 displayed the lowest $\mathrm{MIC}_{50}$ and $\mathrm{MIC}_{90}$ values compared to fluconazole, voriconazole, posaconazole, itraconazole, and VT-1161 against the clinical isolates tested. More importantly, VT-1598 MICs often remained unchanged from its baseline measurement against SC5314 and other fluconazole-susceptible clinical isolates even against isolates containing known resistance mechanisms, indicating that this tetrazole may retain activity against isolates that are normally less susceptible to other azole antifungals. This included multiple fluconazole-resistant isolates with various combinations of $C D R 1, M D R 1$ and ERG11 expression increases and mutations in the ERG11 gene.

Interestingly, when tested against laboratory strains containing individual azole resistance mechanisms, VT-1598 MICs changed relatively little. Traditional azole resistance mechanisms such as efflux pump overexpression (Cdr1 and Mdr1) and overexpression of the azole target (Erg11) did not alter VT-1598 MICs within the concentration ranges tested here. While it is possible that testing lower concentrations might reveal differences in MIC, the clinical relevance at such low concentrations is questionable. Our current finding suggests that these mechanisms individually are not sufficient to confer resistance to VT-1598. Previously, the Tyr132 and Lys143 substitutions in Erg11 were reported to have the strongest individual effects on fluconazole and voriconazole MICs (139). The combination substitutions Y132F\&K143R and Y132F\&F145L, which have been shown to have some of the strongest increases in fluconazole and voriconazole MIC, respectively, did not appreciably change the MIC of VT-1598. Against VT-1161, both these double substitutions showed less of an effect than the single amino acid substitution Y132F. The K143R substitution is thought to alter the H-bond strength of the heme ring propionates 
of the C. albicans $\operatorname{Erg} 11$ protein, which may possibly interfere with the coordination of the azole ring nitrogen and the iron of the CYP51 heme group, and the F145L amino acid substitution is located on the Erg11 proximal surface, allowing possible interactions with NADPH-cytochrome P450 reductase $(139,140)$. Based on the crystal structure of the $C$. albicans CYP51 enzyme complexed with VT-1161, the Y132F substitution is thought to altogether abolish one of six H-bonds between VT-1161 and the protoporphyrin IX propionates on Erg11 (140). It is possible that the Y132F substitution is more important to VT-1161 resistance than either K143R or F145L and that combination mutations might interfere with the primary Y132F substitution, thus leading to the differences in the observed VT-1161 MICs.

Against a collection of predominantly fluconazole-resistant clinical isolates, VT1161 showed good activity, though its individual $\mathrm{MIC}_{50}$ and $\mathrm{MIC}_{90}$ were higher compared to VT-1598. In contrast to VT-1598, the potency of VT-1161 appeared to be more affected by the presence of CDRI and MDR1 overexpression and ERG11 mutations. This was supported by the significant positive correlation established between CDR1/MDR1 expression and VT-1161 MIC in C. albicans clinical isolates.

Additionally, susceptibility testing in strains containing individual mechanisms of azole resistance, wherein the $C D R 1$-overexpressing strains SCTAC1GAD1A and -B and the $M D R 1$-overexpressing strains SCMRR1GAD1A and -B demonstrated increased VT1161 MICs, further supports Mrr1 and Tac1 as mediators of resistance to VT-1161, at least in part through increased production of the transporters Mdr1 and Cdr1, respectively. The recent work by Monk et al. also demonstrated that both the Cdr1 and Mdr1 efflux pumps reduced the effectiveness of VT-1161, and activity against Cdr1- and Mdr1-overexpressing isolates could be restored via the Cdr1- and Mdr1-specific inhibitors RC21v3 and MCC1189, respectively (230). The Erg11 amino acid substitutions Y132F, Y132F\&K143R, and Y132F\&F145L also resulted in shifts in VT$1161 \mathrm{MIC}$ and may contribute to VT-1161 resistance. However, VT-1161 retained activity against a number of isolates with known azole resistance mechanisms. The tested ERG11 mutant strains containing the Y132H, K143R, F145L, E266D, D278N, S405F, G448E, F449V, G450E, G464S, and D466E single substitutions and the D278N\&G464S, and G450E\&I483V double substitutions showed little change in VT1161 MICs compared to the susceptible parent strain SC5314. Thus, VT-1161 has potential to be a future treatment option of azole-resistant $C$. albicans infections or recurrent infections previously treated with older members of the azole class.

Within the five clinical isolates that displayed greatly reduced susceptibility to VT-1161, VT-1598, and the other commercially available triazoles tested, one isolate contained an early stop codon at Trp131 within the ERG3 gene, which encodes for sterol $\Delta 5,6$-desaturase and is critical for ergosterol biosynthesis in C. albicans. It has been previously reported that mutations in $E R G 3$ can result in azole resistance and alternative sterol biosynthesis by avoidance of accumulation of toxic sterol intermediates through defective desaturase enzyme $(142,145,147)$. The inhibition of the azole target $14 \alpha-$ lanosterol demethylase causes accumulation of the toxic sterol intermediate, $14 \alpha-$ methylergosta-8,24(28)-dien-3 $\beta, 6 \alpha$-diol, which is thought to be the source of the fungistatic effect seen with azole antifungal class $(71,72,224)$. However, dysfunctional 
Erg3 results in alternative sterol usage and an inability to produce this toxic intermediate. Therefore, mechanisms that result in a non-functional Erg3 might render an isolate resistant to the azole antifungal drug class, as is seen in the case of the isolate containing the premature stop codon in ERG3.

In summary, the in vitro activity of VT-1161 and VT-1598 against azole-resistant C. albicans clinical isolates and strains with known azole resistance mechanisms suggests that they may prove useful against resistant $C$. albicans infections. Furthermore, given their low and relatively unchanged MICs against many azole-resistant strains, it is possible that VT-1161 and VT-1598 may fill some of the gaps in coverage against azoleresistant isolates. This, in combination with the potential for favorable safety and drug interaction profiles, makes VT-1161 and VT-1598 attractive options as alternative therapies for azole-resistant C. albicans infections. However, the presence of strongly resistant isolates, such as the five clinical isolates with greatly increased MICs to all azoles tested here, suggests the existence of azole-resistance determinants that can provide obstacles to the successful utilization of all azoles, including these new tetrazoles. Further investigation should be undertaken to identify the mechanism(s) responsible for resistance to these agents.

\section{Materials and Methods}

\section{Isolate and strain growth conditions}

Sixty-eight clinical C. albicans isolates were obtained from the University of Iowa. C. albicans isolates and strains were cultured from $-80^{\circ} \mathrm{C}$ freezer stock $(40 \%$ glycerol in YPD media) onto YPD-agar plates overnight at $30^{\circ} \mathrm{C}$. Colonies from YPDagar plates were then either streaked onto Sabouraud Dextrose agar for azole susceptibility testing or grown in liquid YPD media and incubated overnight at $30^{\circ} \mathrm{C}$ for preparation of genomic DNA.

\section{Construction of $C$. albicans strains}

Table 5-2 lists the constructed strains used in this study. Ten single ERG11 mutations and four double mutations were selected from previous studies (139). Two additional strains expressing the Y132H and D278N ERG11 gene mutations were created in a previous study utilizing the $S A T$ flipper cassette $(141,202)$. Briefly, to create the mutant strain SCERG11R1S1C1, ERG11 gene fragments were generated by primers pairs CaERG11_1F with CaERG11SOE-3R_Y132H and CaERG11SOE2F_Y132H with CaERG11_4R using SC5314 template genomic DNA (Table B-3). Short-overlapping extension PCR was used to fuse the resulting ERG11 gene fragments using nested primers CaERG11-AF_(ApaI) and CaERG11-BR_(XhoI). For mutant strain SCERG11R3S3C1, ERG11 gene fragments were generated by primers pairs CaERG11_1F with CaERG11SOE-6R using template genomic DNA from clinical 
Table 5-2. Constructed strains used in Chapter 5

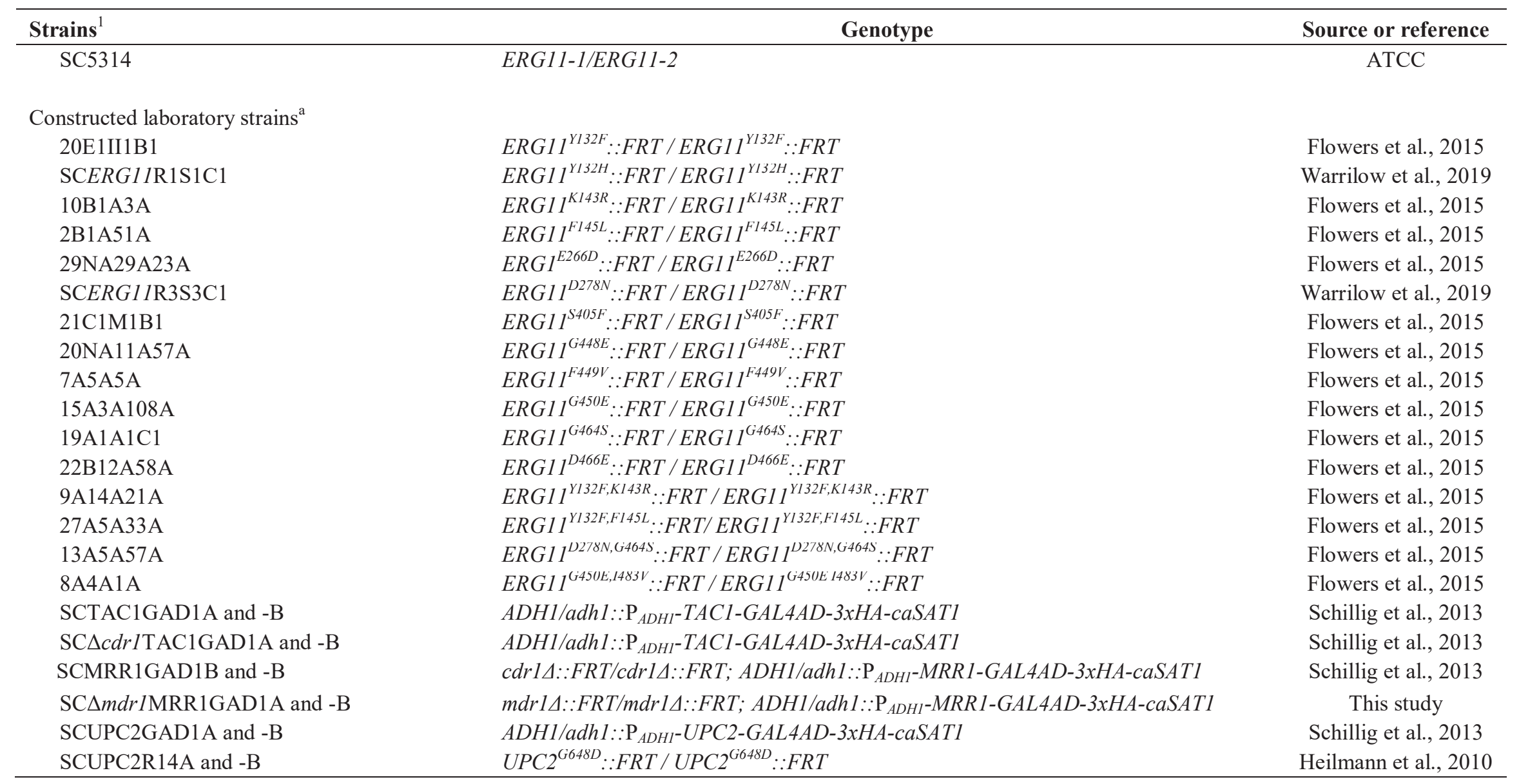

${ }^{1}$ All laboratory strains have SC5314 as background. 
isolate 43 and CaERG11SOE-5F with CaERG11_4R using SC5314 genomic DNA. The resulting fragments were again fused into a single fragment containing either the D278N-containing mutant ERG11 open reading frame (ORF) using nested primers CaERG11-AF_(ApaI) and CaERG11-BR_(XhoI). In constructing the plasmids used in the transformation of both strains, the $E R G 113$ ' flanking sequence was amplified from SC5314 genomic DNA and primers CaERG11_C-F' and CaERG11_D-R'. The inserts 3 ' of the ERG11 ORF were digested with restriction enzymes NotI and SacII, and cloned into the pSFS2-derived plasmid pBSS2 previously described by Vasicek et al. containing the $S A T 1$ flipper cassette from Reu $\beta$ et al. $(202,217)$ to create plasmid pERG11CD. The ERG11 ORF-containing fragments with either the Y132H or D278N mutations were digested using restriction enzymes ApaI and XhoI, gel excised, and cloned into the plasmid pERG11CD to generate plasmids pERG11A1 and pERG11A3. Plasmids were digested with restriction enzymes ApaI and SacII and used to transform strain SC5314 by electroporation to generate heterozygous ERG11 mutants. Recycling of the nourseothricin selection marker through 24 hours of growth in YPD and repeat transformation of the resultant strains generated the homozygous ERG11 allele replacements SCERG11R1S1C1 and SCERG11R3S3C1, confirmed via Southern hybridization and Sanger sequencing. The artificially-activated Tac1, Mrr1, and Upc2 mutants used in this study as well as SC $\Delta c d r 1 \mathrm{TAC} 1 \mathrm{GAD} 1 \mathrm{~A}$ and -B, containing the artificially activated $T A C 1$ allele in a $c d r 1 \triangle$ background, were described in a previous study (117). Strains SC $\Delta m d r 1$ MRR1GAD1A and -B were constructed by introducing the artificially activated $M R R 1$ allele from plasmid pMRR1-GAD1 (32) into the $m d r l \Delta$ mutants SCMDR1M4A and -B (112), respectively.

\section{ERG11 amplification and sequencing}

Table B-3 lists the primers used for ERG11 amplification and sequence verification. The ERG11 ORF of each isolate was PCR amplified from genomic DNA

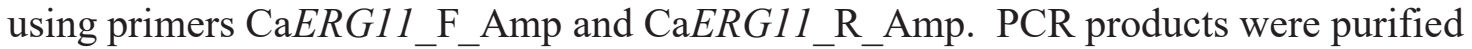
using the QIAquick ${ }^{\circledR}$ PCR Purification Kit (Qiagen) and product was sequenced on an ABI 3130XL genetic analyzer using sequencing primers. Sequencing was accomplished in duplicate in independently grown isolates.

\section{Relative gene expression by real-time PCR}

Expression levels of the genes $C D R 1, M D R 1$, and ERG11 in clinical C. albicans isolates were measured in a previous study, and $C D R 1, M D R 1$, and $E R G 11$ expression of laboratory strains were measured similarly using previously described methods (115). Briefly, first-strand cDNA was generated from $1 \mu \mathrm{g}$ of extracted RNA for each strain using the SuperScript ${ }^{\circledR}$ VILO ${ }^{\text {TM }}$ Master Mix (Invitrogen) reaction kit. Quantitative PCRs were performed on the StepOnePlus ${ }^{\text {TM }}$ Real-Time PCR System (Applied Biosystems) in technical and biological triplicate for each sample. SYBR green PCR master mix (Applied Biosystems) was used for amplification detection of candidate genes against the CaACT1 normalizing gene. Calculation of the relative quantitation values of 
$C D R 1, E R G 11$, and $M D R 1$ gene expression was accomplished using the StepOne Software v2.3 (Applied Biosystems). Primers used in the amplification of genes measured via qPCR are listed in Table B-3.

\section{Susceptibility testing}

Minimum inhibitory concentrations (MIC) of VT-1161, VT-1598, fluconazole, voriconazole, posaconazole, and itraconazole were measured using broth microdilution methods in accordance with the Clinical Laboratory and Standards Institute (200, 201). 96-well microtiter plates containing RPMI-1640 media (0.165M MOPS, with Lglutamine, without sodium bicarbonate, $\mathrm{pH}$ 7.0) were used to incubate strains across serially-diluted concentrations of the each azole. Concentrations ranged from 0.015 to 8 $\mu \mathrm{g} / \mathrm{mL}$ for VT-1161 and VT-1598, 0.125 to $64 \mu \mathrm{g} / \mathrm{mL}$ for fluconazole, and 0.03 to 16 $\mu \mathrm{g} / \mathrm{mL}$ for voriconazole, posaconazole, and itraconazole. MICs were visually read at 24 hours post-incubation at $35^{\circ} \mathrm{C}$ as the minimum concentration required to reduce growth of cells by approximately $50 \%$ or greater compared to drug-free control wells. MICs were performed in duplicate for clinical isolates, ERG11 mutant strains and laboratory strains SCTAC1GAD1A and -B, SC $\Delta c d r 1$ TAC1GAD1A and -B, SCMRR1GAD1A and $\mathrm{B}, \mathrm{SC} \Delta m d r 1 \mathrm{MRR} 1 \mathrm{GAD} 1 \mathrm{~A}$ and $-\mathrm{B}$ and SCUPC2GAD1A and - $\mathrm{B}$. When reporting MICs for strains and isolates, the higher of the MICs was used in this analysis (Table B-1 and B-2), though 98\% (592/606) of MIC duplicate readings were identical or within a single dilution of each other. The geometric mean MIC (GM MIC), $\mathrm{MIC}_{50}$, and $\mathrm{MIC}_{90}$ was reported for clinical $C$. albicans isolates for each triazole and tetrazole agent used in this study. The $\mathrm{MIC}_{50}$ and $\mathrm{MIC}_{90}$ values reported for VT-1161, VT-1598, fluconazole, voriconazole, itraconazole, and posaconazole were defined as the minimum drug concentrations required to inhibit $50 \%$ and $90 \%$ of the clinical C. albicans isolates tested, respectively.

\section{Statistical analysis}

A point-biserial correlation or phi coefficient was used for all continuous and dichotomous variables, respectively, to identify possible predictors of azole resistance. For all statistical tests, a $p$-value less than 0.05 was considered significant. Statistical calculations were performed using IBM $^{\circledR}$ SPSS $^{\circledR}$ analytical software, version 23.

\section{Transparency Declaration}

NPW has received research support to the UT Health San Antonio from Astellas, bioMerieux, Cidara, F2G, Merck, and Viamet, and has served on advisory boards for Merck, Astellas, Toyama, and Viamet. CMY, RJS, and EPG are employees of Viamet Pharmaceuticals, Inc. All other authors have no conflicts of interest. 


\section{CHAPTER 6. CONCLUSIONS}

\section{Introduction}

Candida albicans is the leading etiologic cause of Candida-related infection. While azole-resistance rates in C. albicans remains relatively low, treatment failures still occur resulting from the emergence of highly azole-resistant $C$. albicans isolates. For the foreseeable future, fluconazole and other azole antifungals will continue to be among the most commonly used and prescribed antifungal medications. However, the increased use of this drug class in combination with imperfect clinical practice with antifungal drug dosing will increase risk of azole-resistance development. As has been discussed, $C$. albicans has several known mechanisms enabling it to survive in the presence of azoles, and commonly it is the combination of these resistance mechanisms that arise in clinical isolates that permit them to thrive, even with appropriate treatment. The main objective of my dissertation has been to investigate additional possible mechanisms of azole resistance and resistance-adaptation in C. albicans from mutations derived from a collection of predominantly azole-resistant clinical isolates. Amino acids substitutions in the zinc cluster transcription factor Mrr2, and the sterol biosynthetic proteins Erg11 and Erg3 were the primary focus of this investigation. Furthermore, the novel tetrazole compounds VT-1161 and VT-1598 proved to be valuable agents to screen for unknown azole resistance mechanisms present within this collection. Here, I discuss and interpret the results of each aim of my investigation.

\section{Summary and Conclusions of Chapter 2}

In Chapter 2, I sequenced the MRR2 gene in 57 C. albicans clinical isolates with elevated fluconazole MICs, and identified 15 amino acid substitutions in resistant isolates. Four of these substitutions (S466L, A468G, S469T, T470N) had been previously reported to reduce fluconazole susceptibility through increased expression of the efflux pump encoding gene CDRI (118). Interestingly, these four amino acid substitutions were present in clinical isolates that did not possess significantly increased expression of $C D R 1$ relative to a composite of susceptible isolates. This initially brought into question the validity of the claim that these amino acid substitutions impacted CDRI expression and required further investigation.

I tested the effects of these combined substitutions in Mrr2 as well as the novel substitutions R45Q, A459T, V486M, and V582L by placing the alleles of clinical isolates containing the MRR2 mutations into the native MRR2 locus of the fluconazolesusceptible parent SC5314. Broth microdilution assay and qPCR did not reveal any increases in either fluconazole resistance or $C D R 1$ expression among any of my created strains. Previous work looking at either artificially hyperactive Mrr2 and clinicallyderived mutant Mrr2 increased $C D R 1$ expression and fluconazole resistance $(117,118)$. However, in both cases, the created strains artificially overexpressed the $M R R 2$ gene to observe these effects. I therefore constructed C. albicans strains overexpressing either 
wild type $M R R 2$ or a replica of the $M R R 2$ gene containing mutations previously reported to show the strongest increase in fluconazole MIC. Even with strong expression of $M R R 2$, no change in CDR1 expression or fluconazole MIC compared to SC5314 was observed.

Lastly, by creating an artificially activated $M R R 2$ strain with native promoter expression. I showed two things. Firstly, artificial activation of $M R R 2$ does not result in auto-upregulation of the MRR2 gene. The ZCF genes UPC2 and TAC1 both have been shown to be target genes of their respective proteins, and their expression is upregulated through gain-of-function activity $(93,231)$. Our data suggests that activated Mrr2 does not do this, though further investigation would be required. Secondly, native expression levels of artificially activated $M R R 2$ is sufficient to confer increased $C D R 1$ expression and fluconazole MIC. This suggests that any activating effects from $M R R 2$ mutations should be observable in our created strains expressing mutant MRR2 from the native promoter. The lack of any change in MIC in these strains coupled with the unchanged expression of $C D R 1$ suggests that these mutations are not tentative gain-of-function mutations in $M R R 2$, and more importantly, that they are not clinically relevant for fluconazole resistance.

Though this is ultimately a negative finding, I strongly emphasize the importance of these results. A far-reaching goal of my research is to impact and improve patient outcomes in those with fungal infections treated with the azoles. To this end, discovery and improvement in the established knowledge of fungal pathogens and resistance within these organisms is critical. Publishing contrasting findings to previously established results is important in shaping and correcting the constantly evolving body of scientific knowledge, from which new discoveries and advancements in treatments and therapy is derived. Currently, there are published reported referencing mutations in MRR2 as a relevant mechanism of azole resistance $(160,197,198)$. My findings run strongly to the contrary of these published findings and are therefore important to disseminate in the interest of my research goals.

\section{Summary and Conclusions of Chapter 3}

My own research in Chapter 3 was limited to the creation of the additional Erg11 mutant strains containing the D278N and $\mathrm{Y} 132 \mathrm{H}$ amino acid substitutions, and the analysis of the susceptibility testing data of these new additions to our collection of Erg11 mutant strains. Since ERG11 mutations play a large role in azole resistance, the continued investigation characterizing additional mutations and their effects and interactions in the C. albicans cell remains critically important. My work was contributory to a collaborative research aim to investigate these effects.

The results of the investigation showed that Erg 11 mutants vary considerably in their baseline activities as well as their activity in the presence of the azoles (141). Furthermore, the increased ability to retain activity around the azoles did not, however, always correlate with decreased binding to the azole. Interestingly, these biochemical 
effects did not always translate into what was seen in $C$. albicans in vitro susceptibility testing of the ERG11 mutations. This suggests that some ERG11 mutations may work to alter interaction with other components, such as NADPH-cytochrome P450 reductase, that work with CaCYP51 in sterol biosynthesis. Overall, my work in combination with the biochemical data on these Erg11 mutants shed additional light on the mode of action by which Erg11 amino acid substitutions interfere with growth inhibition by the azoles. It also identifies the possibility that these substitutions may impact normal cell sterol biosynthesis production due to the altered catalytic rates exhibited by the Erg11 mutant proteins. This notion was important in the justification of my investigation of another sterol biosynthesis gene, ERG3, which is detailed next.

\section{Summary and Conclusions of Chapter 4}

In chapter 4, I sequenced the $E R G 3$ gene of our research group's collection of clinical C. albicans isolates in an initial effort to define additional azole resistance mechanisms present in the collection. We had previously reported the majority of the major resistance mechanisms present among these isolates (115). However, sterol profiles on this collection identified isolates with lower ergosterol content. ERG3 mutants resulting in defective C-5 sterol desaturase have been reported as having little to no ergosterol content and increased amounts of ergosta-7,22-dienol instead (224). One such clinical isolates matched this "textbook" ERG3 mutant phenotype, and subsequent sequencing of the open-reading frame confirmed a premature stop codon at the normal Trp-131 residue. Interestingly, my sequence work identified multiple ERG3 mutations present in these clinical isolates. Investigating whether these mutations may have any role in azole resistance, I noticed the high co-occurrence of the A351V ERG3 mutation with ERG11 mutations.

Given our suspicion that ERG11 mutations may come at a fitness cost and may require possible compensatory mutations in order to adapt, I proceeded to make $E R G 3$ and $E R G 11$ combination mutants to test whether ERG3 had an indirect role in azole resistance. Unfortunately, growth competition and fitness testing between ERG11 mutants with and without mutant ERG3 could not be completed since I was unsuccessful in creating the G307S and Y132H, K143R Erg11 mutants. Based on data from our collaborators, these two sets of amino acid substitutions conferred the poorest catalytic turnover rates for the Erg11 enzyme, and would be expected to show the greatest fitness

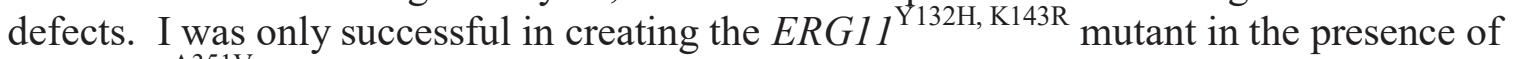
the $E R G 3^{\mathrm{A} 351 \mathrm{~V}}$ mutation. While not conclusive, it is preliminary evidence that perhaps the ERG3 ${ }^{\mathrm{A} 351 \mathrm{~V}}$ mutation may confer a compensatory fitness benefit on C. albicans isolates that accrue multiple ERG11 mutations.

While further work needs to be done to investigate any possible beneficial effects for the cell, the possibility of mutations in ERG3 conferring a compensatory fitness benefit raises interesting questions about azole resistance in clinical isolates. If certain $E R G 3$ mutations are acquired early on by the cell, then the mutagenic potential of the ERG11 gene, and therefore of azole resistance development, could increase as well. The 
identification of such an evolution of azole resistance could make $E R G 3$ mutations an intriguing diagnostic marker in the prediction of azole treatment failures through acquired resistance.

\section{Summary and Conclusions of Chapter 5}

Lastly, my work in Chapter 5 involved the analysis of the susceptibilities of our C. albicans clinical isolates and resistant laboratory strains to the established azole antifungals used in invasive fungal infection alongside the new tetrazole antifungal agents VT-1161 and VT-1598. Both newer agents showed potent activity against predominantly azole-resistant strains and isolates. In particular, VT-1598 displayed activity against the vast majority of clinical isolates as well as strains overexpressing $C D R 1, M D R 1$, or ERG11 through gain-of-function ZCF mutations. In comparison, the currently available antifungal agent posaconazole displayed similar activity to VT-1598, while voriconazole and itraconazole were more comparable VT-1161's activity against clinical isolates. Both tetrazoles also appeared to be unaffected by several, but not all, ERG11 mutations, similar to the triazole posaconazole. Overall, the tetrazole compounds appear to be promising agents that could be utilized in the future as alternative therapies in invasive candidiasis.

An important finding in the analysis of this susceptibility data is the identification of isolates 55, 56, 58, 60, and 65 that all showed greatly increased MICs to the tetrazoles and all other azoles. Apart from isolate 60 , which possesses an early stop codon in $E R G 3$, the other isolates do not possess any unique traits with respect to known azole resistance mechanisms that sets them apart from other clinical isolates. This indicates that these isolates possess as yet undiscovered mechanisms of azole resistance and provides and interesting future project to investigate these isolates further.

\section{A Final Word}

The findings of my research projects leave several questions that remain to be answered in C. albicans. Whether mutations in additional ZCFs may play a role in azole resistance is currently an ongoing investigation. The role of compensatory mutations in $E R G 3$ and other sterol biosynthesis genes is a plausible but as yet unproven mechanism that could influence azole resistance development. Do mutations in ERG11 also alter CaCYP51 interaction with peripheral components outside of the azole antifungals that may influence susceptibility? And what are the means by which some clinical isolates possess such strongly reduced susceptibility to the azole antifungals, if not by any of the currently known mechanisms? One can speculate that some of these questions may ultimately prove to be important in changing the clinical approach to dealing with azoleresistant Candida. For the immediate future, though, we must remember to put one foot in front of the other - assessing the need for further investigation into these ever-shifting questions based on the research we gather: one gel, one blot, one cell at a time. 


\section{LIST OF REFERENCES}

1. Hoffmann C, Dollive S, Grunberg S, Chen J, Li H, Wu GD, Lewis JD, Bushman FD. 2013. Archaea and fungi of the human gut microbiome: correlations with diet and bacterial residents. PLoS One 8:e66019.

2. Ghannoum MA, Jurevic RJ, Mukherjee PK, Cui F, Sikaroodi M, Naqvi A, Gillevet PM. 2010. Characterization of the Oral Fungal Microbiome (Mycobiome) in Healthy Individuals. Plos Pathogens 6.

3. Findley K, Oh J, Yang J, Conlan S, Deming C, Meyer JA, Schoenfeld D, Nomicos E, Park M, Program NIHISCCS, Kong HH, Segre JA. 2013.

Topographic diversity of fungal and bacterial communities in human skin. Nature 498:367-70.

4. Merenstein D, Hu H, Wang C, Hamilton P, Blackmon M, Chen H, Calderone R, Li D. 2013. Colonization by Candida species of the oral and vaginal mucosa in HIV-infected and noninfected women. AIDS Res Hum Retroviruses 29:30-4.

5. Arendrup MC, Patterson TF. 2017. Multidrug-Resistant Candida: Epidemiology, Molecular Mechanisms, and Treatment. J Infect Dis 216:S445-S451.

6. Muskett H, Shahin J, Eyres G, Harvey S, Rowan K, Harrison D. 2011. Risk factors for invasive fungal disease in critically ill adult patients: a systematic review. Crit Care 15:R287.

7. Diekema D, Arbefeville S, Boyken L, Kroeger J, Pfaller M. 2012. The changing epidemiology of healthcare-associated candidemia over three decades. Diagn Microbiol Infect Dis 73:45-8.

8. Edmond MB, Wallace SE, McClish DK, Pfaller MA, Jones RN, Wenzel RP. 1999. Nosocomial bloodstream infections in United States hospitals: A three-year analysis. Clinical Infectious Diseases 29:239-244.

9. Kullberg BJ, Oude Lashof AM. 2002. Epidemiology of opportunistic invasive mycoses. Eur J Med Res 7:183-91.

10. Bongomin F, Gago S, Oladele RO, Denning DW. 2017. Global and MultiNational Prevalence of Fungal Diseases-Estimate Precision. J Fungi (Basel) 3.

11. Lamoth F, Lockhart SR, Berkow EL, Calandra T. 2018. Changes in the epidemiological landscape of invasive candidiasis. J Antimicrob Chemother 73 :i4-i13. 
12. Wisplinghoff H, Bischoff T, Tallent SM, Seifert H, Wenzel RP, Edmond MB. 2004. Nosocomial bloodstream infections in US hospitals: analysis of 24,179 cases from a prospective nationwide surveillance study. Clin Infect Dis 39:30917.

13. Kett DH, Azoulay E, Echeverria PM, Vincent JL, Extended Prevalence of Infection in ICUSGoI. 2011. Candida bloodstream infections in intensive care units: analysis of the extended prevalence of infection in intensive care unit study. Crit Care Med 39:665-70.

14. Whaley SG, Berkow EL, Rybak JM, Nishimoto AT, Barker KS, Rogers PD. 2016. Azole Antifungal Resistance in Candida albicans and Emerging Nonalbicans Candida Species. Front Microbiol 7:2173.

15. Castanheira M, Messer SA, Rhomberg PR, Pfaller MA. 2016. Antifungal susceptibility patterns of a global collection of fungal isolates: results of the SENTRY Antifungal Surveillance Program (2013). Diagn Microbiol Infect Dis 85:200-4.

16. Goncalves B, Ferreira C, Alves CT, Henriques M, Azeredo J, Silva S. 2016. Vulvovaginal candidiasis: Epidemiology, microbiology and risk factors. Crit Rev Microbiol 42:905-27.

17. Pappas PG, Lionakis MS, Arendrup MC, Ostrosky-Zeichner L, Kullberg BJ. 2018. Invasive candidiasis. Nat Rev Dis Primers 4:18026.

18. Tan BH, Chakrabarti A, Li RY, Patel AK, Watcharananan SP, Liu Z, Chindamporn A, Tan AL, Sun PL, Wu UI, Chen YC, Asia Fungal Working G. 2015. Incidence and species distribution of candidaemia in Asia: a laboratorybased surveillance study. Clin Microbiol Infect 21:946-53.

19. Cheng YR, Lin LC, Young TG, Liu CE, Chen CH, Tsay RW. 2006. Risk factors for candidemia-related mortality at a medical center in central Taiwan. J Microbiol Immunol Infect 39:155-61.

20. Colombo AL, Nucci M, Park BJ, Nouer SA, Arthington-Skaggs B, da Matta DA, Warnock D, Morgan J, Brazilian Network Candidemia S. 2006. Epidemiology of candidemia in Brazil: a nationwide sentinel surveillance of candidemia in eleven medical centers. J Clin Microbiol 44:2816-23.

21. Doi AM, Pignatari AC, Edmond MB, Marra AR, Camargo LF, Siqueira RA, da Mota VP, Colombo AL. 2016. Epidemiology and Microbiologic Characterization of Nosocomial Candidemia from a Brazilian National Surveillance Program. PLoS One 11:e146909. 
22. Chowdhary A, Becker K, Fegeler W, Gugnani HC, Kapoor L, Randhawa VS, Mehta G. 2003. An outbreak of candidemia due to Candida tropicalis in a neonatal intensive care unit. Mycoses 46:287-92.

23. Farooqi JQ, Jabeen K, Saeed N, Iqbal N, Malik B, Lockhart SR, Zafar A, Brandt ME, Hasan R. 2013. Invasive candidiasis in Pakistan: clinical characteristics, species distribution and antifungal susceptibility. J Med Microbiol 62:259-68.

24. Hachem R, Hanna H, Kontoyiannis D, Jiang Y, Raad I. 2008. The changing epidemiology of invasive candidiasis: Candida glabrata and Candida krusei as the leading causes of candidemia in hematologic malignancy. Cancer 112:2493-9.

25. Sipsas NV, Lewis RE, Tarrand J, Hachem R, Rolston KV, Raad, II, Kontoyiannis DP. 2009. Candidemia in patients with hematologic malignancies in the era of new antifungal agents (2001-2007): stable incidence but changing epidemiology of a still frequently lethal infection. Cancer 115:4745-52.

26. Chowdhary A, Sharma C, Meis JF. 2017. Candida auris: A rapidly emerging cause of hospital-acquired multidrug-resistant fungal infections globally. PLoS Pathog 13:e1006290.

27. Nobile CJ, Johnson AD. 2015. Candida albicans Biofilms and Human Disease. Annu Rev Microbiol 69:71-92.

28. Pfaller MA, Diekema DJ. 2007. Epidemiology of invasive candidiasis: a persistent public health problem. Clin Microbiol Rev 20:133-63.

29. Pappas PG, Kauffman CA, Andes DR, Clancy CJ, Marr KA, Ostrosky-Zeichner L, Reboli AC, Schuster MG, Vazquez JA, Walsh TJ, Zaoutis TE, Sobel JD. 2016. Clinical Practice Guideline for the Management of Candidiasis: 2016 Update by the Infectious Diseases Society of America. Clin Infect Dis 62:e1-50.

30. Pfaller MA, Diekema DJ, Gibbs DL, Newell VA, Ellis D, Tullio V, Rodloff A, Fu W, Ling TA, Global Antifungal Surveillance G. 2010. Results from the ARTEMIS DISK Global Antifungal Surveillance Study, 1997 to 2007: a 10.5year analysis of susceptibilities of Candida Species to fluconazole and voriconazole as determined by CLSI standardized disk diffusion. J Clin Microbiol 48:1366-77.

31. Lyon GM, Karatela S, Sunay S, Adiri Y, Candida Surveillance Study I. 2010. Antifungal susceptibility testing of Candida isolates from the Candida surveillance study. J Clin Microbiol 48:1270-5.

32. Pfaller MA, Moet GJ, Messer SA, Jones RN, Castanheira M. 2011. Candida bloodstream infections: comparison of species distributions and antifungal resistance patterns in community-onset and nosocomial isolates in the SENTRY 
Antimicrobial Surveillance Program, 2008-2009. Antimicrob Agents Chemother 55:561-6.

33. Pfaller MA, Espinel-Ingroff A, Canton E, Castanheira M, Cuenca-Estrella M, Diekema DJ, Fothergill A, Fuller J, Ghannoum M, Jones RN, Lockhart SR, Martin-Mazuelos E, Melhem MS, Ostrosky-Zeichner L, Pappas P, Pelaez T, Peman J, Rex J, Szeszs MW. 2012. Wild-type MIC distributions and epidemiological cutoff values for amphotericin B, flucytosine, and itraconazole and Candida spp. as determined by CLSI broth microdilution. J Clin Microbiol 50:2040-6.

34. Lockhart SR, Iqbal N, Cleveland AA, Farley MM, Harrison LH, Bolden CB, Baughman W, Stein B, Hollick R, Park BJ, Chiller T. 2012. Species identification and antifungal susceptibility testing of Candida bloodstream isolates from population-based surveillance studies in two U.S. cities from 2008 to 2011. J Clin Microbiol 50:3435-42.

35. Moyes DL, Wilson D, Richardson JP, Mogavero S, Tang SX, Wernecke J, Hofs S, Gratacap RL, Robbins J, Runglall M, Murciano C, Blagojevic M, Thavaraj S, Forster TM, Hebecker B, Kasper L, Vizcay G, Iancu SI, Kichik N, Hader A, Kurzai O, Luo T, Kruger T, Kniemeyer O, Cota E, Bader O, Wheeler RT, Gutsmann T, Hube B, Naglik JR. 2016. Candidalysin is a fungal peptide toxin critical for mucosal infection. Nature 532:64-8.

36. Kasper L, Konig A, Koenig PA, Gresnigt MS, Westman J, Drummond RA, Lionakis MS, Gross O, Ruland J, Naglik JR, Hube B. 2018. The fungal peptide toxin Candidalysin activates the NLRP3 inflammasome and causes cytolysis in mononuclear phagocytes. Nat Commun 9:4260.

37. Moyes DL, Richardson JP, Naglik JR. 2015. Candida albicans-epithelial interactions and pathogenicity mechanisms: scratching the surface. Virulence 6:338-46.

38. Taschdjian CL, Burchall JJ, Kozinn PJ. 1960. Rapid identification of Candida albicans by filamentation on serum and serum substitutes. AMA J Dis Child 99:212-5.

39. Simonetti N, Strippoli V, Cassone A. 1974. Yeast-mycelial conversion induced by N-acetyl-D-glucosamine in Candida albicans. Nature 250:344-6.

40. Buffo J, Herman MA, Soll DR. 1984. A characterization of pH-regulated dimorphism in Candida albicans. Mycopathologia 85:21-30.

41. Berman J, Sudbery PE. 2002. Candida Albicans: a molecular revolution built on lessons from budding yeast. Nat Rev Genet 3:918-30. 
42. Chandra J, Kuhn DM, Mukherjee PK, Hoyer LL, McCormick T, Ghannoum MA. 2001. Biofilm formation by the fungal pathogen Candida albicans: development, architecture, and drug resistance. J Bacteriol 183:5385-94.

43. Fox EP, Nobile CJ. 2012. A sticky situation: untangling the transcriptional network controlling biofilm development in Candida albicans. Transcription $3: 315-22$.

44. Ramage G, Mowat E, Jones B, Williams C, Lopez-Ribot J. 2009. Our current understanding of fungal biofilms. Crit Rev Microbiol 35:340-55.

45. Ramage G, Saville SP, Thomas DP, Lopez-Ribot JL. 2005. Candida biofilms: an update. Eukaryot Cell 4:633-8.

46. Lan CY, Newport G, Murillo LA, Jones T, Scherer S, Davis RW, Agabian N. 2002. Metabolic specialization associated with phenotypic switching in Candidaalbicans. Proc Natl Acad Sci U S A 99:14907-12.

47. Moreno-Ruiz E, Galan-Diez M, Zhu W, Fernandez-Ruiz E, d'Enfert C, Filler SG, Cossart P, Veiga E. 2009. Candida albicans internalization by host cells is mediated by a clathrin-dependent mechanism. Cell Microbiol 11:1179-89.

48. Gow NA, van de Veerdonk FL, Brown AJ, Netea MG. 2011. Candida albicans morphogenesis and host defence: discriminating invasion from colonization. Nat Rev Microbiol 10:112-22.

49. Phan QT, Myers CL, Fu Y, Sheppard DC, Yeaman MR, Welch WH, Ibrahim AS, Edwards JE, Jr., Filler SG. 2007. Als3 is a Candida albicans invasin that binds to cadherins and induces endocytosis by host cells. PLoS Biol 5:e64.

50. Zhu W, Phan QT, Boontheung P, Solis NV, Loo JA, Filler SG. 2012. EGFR and HER2 receptor kinase signaling mediate epithelial cell invasion by Candida albicans during oropharyngeal infection. Proc Natl Acad Sci U S A 109:14194-9.

51. Holt RJ, Azmi A. 1978. Miconazole-resistant Candida. Lancet 1:50-1.

52. Holt RJ. 1979. Clinical isolates of Canada albicans. Infection 7:154-5.

53. Horsburgh CR, Jr., Kirkpatrick CH. 1983. Long-term therapy of chronic mucocutaneous candidiasis with ketoconazole: experience with twenty-one patients. Am J Med 74:23-9.

54. Warnock DW, Johnson EM, Richardson MD, Vickers CF. 1983. Modified response to ketoconazole of Candida albicans from a treatment failure. Lancet $1: 642-3$. 
55. Smith KJ, Warnock DW, Kennedy CT, Johnson EM, Hopwood V, Van Cutsem J, Vanden Bossche H. 1986. Azole resistance in Candida albicans. J Med Vet Mycol 24:133-44.

56. Lucatorto FM, Franker C, Hardy WD, Chafey S. 1991. Treatment of refractory oral candidiasis with fluconazole. A case report. Oral Surg Oral Med Oral Pathol $71: 42-4$.

57. White A, Goetz MB. 1994. Azole-resistant Candida albicans: report of two cases of resistance to fluconazole and review. Clin Infect Dis 19:687-92.

58. Evans TG, Mayer J, Cohen S, Classen D, Carroll K. 1991. Fluconazole failure in the treatment of invasive mycoses. J Infect Dis 164:1232-5.

59. Cleveland AA, Farley MM, Harrison LH, Stein B, Hollick R, Lockhart SR, Magill SS, Derado G, Park BJ, Chiller TM. 2012. Changes in incidence and antifungal drug resistance in candidemia: results from population-based laboratory surveillance in Atlanta and Baltimore, 2008-2011. Clin Infect Dis $55: 1352-61$.

60. Arendrup MC, Fuursted K, Gahrn-Hansen B, Jensen IM, Knudsen JD, Lundgren B, Schonheyder HC, Tvede M. 2005. Seminational surveillance of fungemia in Denmark: notably high rates of fungemia and numbers of isolates with reduced azole susceptibility. J Clin Microbiol 43:4434-40.

61. Arendrup MC, Bruun B, Christensen JJ, Fuursted K, Johansen HK, Kjaeldgaard P, Knudsen JD, Kristensen L, Moller J, Nielsen L, Rosenvinge FS, Roder B, Schonheyder HC, Thomsen MK, Truberg K. 2011. National surveillance of fungemia in Denmark (2004 to 2009). J Clin Microbiol 49:325-34.

62. Puig-Asensio M, Padilla B, Garnacho-Montero J, Zaragoza O, Aguado JM, Zaragoza R, Montejo M, Munoz P, Ruiz-Camps I, Cuenca-Estrella M, Almirante B, Project C, Geih G, Reipi. 2014. Epidemiology and predictive factors for early and late mortality in Candida bloodstream infections: a population-based surveillance in Spain. Clin Microbiol Infect 20:O245-54.

63. Trouve C, Blot S, Hayette MP, Jonckheere S, Patteet S, Rodriguez-Villalobos H, Symoens F, Van Wijngaerden E, Lagrou K. 2017. Epidemiology and reporting of candidaemia in Belgium: a multi-centre study. Eur J Clin Microbiol Infect Dis 36:649-655.

64. Hesstvedt L, Gaustad P, Andersen CT, Haarr E, Hannula R, Haukland HH, Hermansen NO, Larssen KW, Mylvaganam H, Ranheim TE, Sandven P, Nordoy I, Norwegian Yeast Study G, Kanestrom A, Grub C, Onken A, Thielsen C, Skaare D, Tofteland S, Sonsteby LJ, Hjetland R, Hide R, Vik E, Kummel A, Asheim S. 
2015. Twenty-two years of candidaemia surveillance: results from a Norwegian national study. Clin Microbiol Infect 21:938-45.

65. Chapman B, Slavin M, Marriott D, Halliday C, Kidd S, Arthur I, Bak N, Heath CH, Kennedy K, Morrissey CO, Sorrell TC, van Hal S, Keighley C, Goeman E, Underwood N, Hajkowicz K, Hofmeyr A, Leung M, Macesic N, Botes J, Blyth C, Cooley L, George CR, Kalukottege P, Kesson A, McMullan B, Baird R, Robson J, Korman TM, Pendle S, Weeks K, Liu E, Cheong E, Chen S, Australian, New Zealand Mycoses Interest G. 2017. Changing epidemiology of candidaemia in Australia. J Antimicrob Chemother 72:1103-1108.

66. Govender NP, Patel J, Magobo RE, Naicker S, Wadula J, Whitelaw A, Coovadia Y, Kularatne R, Govind C, Lockhart SR, Zietsman IL, group TR-SA. 2016. Emergence of azole-resistant Candida parapsilosis causing bloodstream infection: results from laboratory-based sentinel surveillance in South Africa. J Antimicrob Chemother 71:1994-2004.

67. Chakrabarti A, Sood P, Rudramurthy SM, Chen S, Kaur H, Capoor M, Chhina D, Rao R, Eshwara VK, Xess I, Kindo AJ, Umabala P, Savio J, Patel A, Ray U, Mohan S, Iyer R, Chander J, Arora A, Sardana R, Roy I, Appalaraju B, Sharma A, Shetty A, Khanna N, Marak R, Biswas S, Das S, Harish BN, Joshi S, Mendiratta D. 2015. Incidence, characteristics and outcome of ICU-acquired candidemia in India. Intensive Care Med 41:285-95.

68. Benedict K, Jackson BR, Chiller T, Beer KD. 2018. Estimation of direct healthcare costs of fungal diseases in the United States. Clin Infect Dis doi:10.1093/cid/ciy776.

69. Askinyte D, Matulionyte R, Rimkevicius A. 2015. Oral manifestations of HIV disease: A review. Stomatologija 17:21-8.

70. Redding SW, Zellars RC, Kirkpatrick WR, McAtee RK, Caceres MA, Fothergill AW, Lopez-Ribot JL, Bailey CW, Rinaldi MG, Patterson TF. 1999. Epidemiology of oropharyngeal Candida colonization and infection in patients receiving radiation for head and neck cancer. J Clin Microbiol 37:3896-900.

71. Watson PF, Rose ME, Ellis SW, England H, Kelly SL. 1989. Defective sterol C56 desaturation and azole resistance: a new hypothesis for the mode of action of azole antifungals. Biochem Biophys Res Commun 164:1170-5.

72. Kelly SL, Lamb DC, Kelly DE, Manning NJ, Loeffler J, Hebart H, Schumacher U, Einsele H. 1997. Resistance to fluconazole and cross-resistance to amphotericin B in Candida albicans from AIDS patients caused by defective sterol delta5,6-desaturation. FEBS Lett 400:80-2. 
73. Rybak JM, Marx KR, Nishimoto AT, Rogers PD. 2015. Isavuconazole: Pharmacology, Pharmacodynamics, and Current Clinical Experience with a New Triazole Antifungal Agent. Pharmacotherapy 35:1037-51.

74. Shirley M, Scott LJ. 2016. Isavuconazole: A Review in Invasive Aspergillosis and Mucormycosis. Drugs 76:1647-1657.

75. Wiederhold NP, Xu X, Wang A, Najvar LK, Garvey EP, Ottinger EA, Alimardanov A, Cradock J, Behnke M, Hoekstra WJ, Brand SR, Schotzinger RJ, Jaramillo R, Olivo M, Kirkpatrick WR, Patterson TF. 2018. In Vivo Efficacy of VT-1129 against Experimental Cryptococcal Meningitis with the Use of a Loading Dose-Maintenance Dose Administration Strategy. Antimicrob Agents Chemother 62.

76. Nishimoto AT, Wiederhold NP, Flowers SA, Zhang Q, Kelly SL, Morschhäuser J, Yates CM, Hoekstra WJ, Schotzinger RJ, Garvey EP, Rogers PD. 2019. In vitro activities of the novel investigational tetrazoles VT-1161 and VT-1598 compared to the triazole antifungals against azole-resistant strains and clinical isolates of Candida albicans. Antimicrobial Agents and Chemotherapy doi:10.1128/aac.00341-19:AAC.00341-19.

77. Prasad R, De Wergifosse P, Goffeau A, Balzi E. 1995. Molecular cloning and characterization of a novel gene of Candida albicans, CDR1, conferring multiple resistance to drugs and antifungals. Curr Genet 27:320-9.

78. Sanglard D, Ischer F, Monod M, Bille J. 1997. Cloning of Candida albicans genes conferring resistance to azole antifungal agents: characterization of CDR2, a new multidrug ABC transporter gene. Microbiology 143 ( Pt 2):405-16.

79. Sanglard D, Kuchler K, Ischer F, Pagani JL, Monod M, Bille J. 1995. Mechanisms of resistance to azole antifungal agents in Candida albicans isolates from AIDS patients involve specific multidrug transporters. Antimicrob Agents Chemother 39:2378-86.

80. White TC. 1997. Increased mRNA levels of ERG16, CDR, and MDR1 correlate with increases in azole resistance in Candida albicans isolates from a patient infected with human immunodeficiency virus. Antimicrob Agents Chemother 41:1482-7.

81. Niimi M, Niimi K, Takano Y, Holmes AR, Fischer FJ, Uehara Y, Cannon RD. 2004. Regulated overexpression of CDR1 in Candida albicans confers multidrug resistance. J Antimicrob Chemother 54:999-1006.

82. Coste AT, Karababa M, Ischer F, Bille J, Sanglard D. 2004. TAC1, transcriptional activator of $\mathrm{CDR}$ genes, is a new transcription factor involved in the regulation of Candida albicans ABC transporters CDR1 and CDR2. Eukaryot Cell 3:1639-52. 
83. Coste A, Turner V, Ischer F, Morschhauser J, Forche A, Selmecki A, Berman J, Bille J, Sanglard D. 2006. A mutation in Tac1p, a transcription factor regulating CDR1 and CDR2, is coupled with loss of heterozygosity at chromosome 5 to mediate antifungal resistance in Candida albicans. Genetics 172:2139-56.

84. Manoharlal R, Gaur NA, Panwar SL, Morschhauser J, Prasad R. 2008. Transcriptional activation and increased mRNA stability contribute to overexpression of CDR1 in azole-resistant Candida albicans. Antimicrob Agents Chemother 52:1481-92.

85. Manoharlal R, Gorantala J, Sharma M, Sanglard D, Prasad R. 2010. PAP1 [poly(A) polymerase 1] homozygosity and hyperadenylation are major determinants of increased mRNA stability of CDR1 in azole-resistant clinical isolates of Candida albicans. Microbiology 156:313-26.

86. Siikala E, Rautemaa R, Richardson M, Saxen H, Bowyer P, Sanglard D. 2010. Persistent Candida albicans colonization and molecular mechanisms of azole resistance in autoimmune polyendocrinopathy-candidiasis-ectodermal dystrophy (APECED) patients. J Antimicrob Chemother 65:2505-13.

87. Sasse C, Schillig R, Dierolf F, Weyler M, Schneider S, Mogavero S, Rogers PD, Morschhauser J. 2011. The transcription factor Ndt80 does not contribute to Mrr1-, Tac1-, and Upc2-mediated fluconazole resistance in Candida albicans. PLoS One 6:e25623.

88. Coste A, Selmecki A, Forche A, Diogo D, Bougnoux ME, d'Enfert C, Berman J, Sanglard D. 2007. Genotypic evolution of azole resistance mechanisms in sequential Candida albicans isolates. Eukaryot Cell 6:1889-904.

89. de Micheli M, Bille J, Schueller C, Sanglard D. 2002. A common drug-responsive element mediates the upregulation of the Candida albicans $\mathrm{ABC}$ transporters CDR1 and CDR2, two genes involved in antifungal drug resistance. Mol Microbiol 43:1197-214.

90. Hernaez ML, Gil C, Pla J, Nombela C. 1998. Induced expression of the Candida albicans multidrug resistance gene CDR1 in response to fluconazole and other antifungals. Yeast 14:517-26.

91. Liu Z, Myers LC. 2017. Mediator Tail Module Is Required for Tac1-Activated CDR1 Expression and Azole Resistance in Candida albicans. Antimicrob Agents Chemother 61.

92. Prasad R, Banerjee A, Khandelwal NK, Dhamgaye S. 2015. The ABCs of Candida albicans Multidrug Transporter Cdr1. Eukaryot Cell 14:1154-64. 
93. Liu TT, Znaidi S, Barker KS, Xu L, Homayouni R, Saidane S, Morschhauser J, Nantel A, Raymond M, Rogers PD. 2007. Genome-wide expression and location analyses of the Candida albicans Tac1p regulon. Eukaryot Cell 6:2122-38.

94. Sanglard D, Coste AT. 2016. Activity of Isavuconazole and Other Azoles against Candida Clinical Isolates and Yeast Model Systems with Known Azole Resistance Mechanisms. Antimicrob Agents Chemother 60:229-38.

95. Xiao L, Madison V, Chau AS, Loebenberg D, Palermo RE, McNicholas PM. 2004. Three-dimensional models of wild-type and mutated forms of cytochrome P450 14alpha-sterol demethylases from Aspergillus fumigatus and Candida albicans provide insights into posaconazole binding. Antimicrob Agents Chemother 48:568-74.

96. Cheng S, Clancy CJ, Nguyen KT, Clapp W, Nguyen MH. 2007. A Candida albicans petite mutant strain with uncoupled oxidative phosphorylation overexpresses MDR1 and has diminished susceptibility to fluconazole and voriconazole. Antimicrob Agents Chemother 51:1855-8.

97. Fling ME, Kopf J, Tamarkin A, Gorman JA, Smith HA, Koltin Y. 1991. Analysis of a Candida albicans gene that encodes a novel mechanism for resistance to benomyl and methotrexate. Mol Gen Genet 227:318-29.

98. Ben-Yaacov R, Knoller S, Caldwell GA, Becker JM, Koltin Y. 1994. Candida albicans gene encoding resistance to benomyl and methotrexate is a multidrug resistance gene. Antimicrob Agents Chemother 38:648-52.

99. Franz R, Kelly SL, Lamb DC, Kelly DE, Ruhnke M, Morschhauser J. 1998. Multiple molecular mechanisms contribute to a stepwise development of fluconazole resistance in clinical Candida albicans strains. Antimicrob Agents Chemother 42:3065-72.

100. Lopez-Ribot JL, McAtee RK, Lee LN, Kirkpatrick WR, White TC, Sanglard D, Patterson TF. 1998. Distinct patterns of gene expression associated with development of fluconazole resistance in serial candida albicans isolates from human immunodeficiency virus-infected patients with oropharyngeal candidiasis. Antimicrob Agents Chemother 42:2932-7.

101. Wirsching S, Michel S, Morschhauser J. 2000. Targeted gene disruption in Candida albicans wild-type strains: the role of the MDR1 gene in fluconazole resistance of clinical Candida albicans isolates. Mol Microbiol 36:856-65.

102. Wirsching S, Michel S, Kohler G, Morschhauser J. 2000. Activation of the multiple drug resistance gene MDR1 in fluconazole-resistant, clinical Candida albicans strains is caused by mutations in a trans-regulatory factor. J Bacteriol 182:400-4. 
103. Harry JB, Oliver BG, Song JL, Silver PM, Little JT, Choiniere J, White TC. 2005. Drug-induced regulation of the MDR1 promoter in Candida albicans. Antimicrob Agents Chemother 49:2785-92.

104. Morschhauser J, Barker KS, Liu TT, Bla BWJ, Homayouni R, Rogers PD. 2007. The transcription factor Mrr1p controls expression of the MDR1 efflux pump and mediates multidrug resistance in Candida albicans. PLoS Pathog 3:e164.

105. Dunkel N, Blass J, Rogers PD, Morschhauser J. 2008. Mutations in the multi-drug resistance regulator MRR1, followed by loss of heterozygosity, are the main cause of MDR1 overexpression in fluconazole-resistant Candida albicans strains. Mol Microbiol 69:827-40.

106. Silver PM, Oliver BG, White TC. 2004. Role of Candida albicans transcription factor Upc2p in drug resistance and sterol metabolism. Eukaryot Cell 3:1391-7.

107. Perea S, Lopez-Ribot JL, Kirkpatrick WR, McAtee RK, Santillan RA, Martinez M, Calabrese D, Sanglard D, Patterson TF. 2001. Prevalence of molecular mechanisms of resistance to azole antifungal agents in Candida albicans strains displaying high-level fluconazole resistance isolated from human immunodeficiency virus-infected patients. Antimicrob Agents Chemother 45:2676-84.

108. MacPherson S, Akache B, Weber S, De Deken X, Raymond M, Turcotte B. 2005. Candida albicans zinc cluster protein Upc2p confers resistance to antifungal drugs and is an activator of ergosterol biosynthetic genes. Antimicrob Agents Chemother 49:1745-52.

109. Oliver BG, Song JL, Choiniere JH, White TC. 2007. cis-Acting elements within the Candida albicans ERG11 promoter mediate the azole response through transcription factor Upc2p. Eukaryot Cell 6:2231-9.

110. Dunkel N, Liu TT, Barker KS, Homayouni R, Morschhauser J, Rogers PD. 2008. A gain-of-function mutation in the transcription factor Upc2p causes upregulation of ergosterol biosynthesis genes and increased fluconazole resistance in a clinical Candida albicans isolate. Eukaryot Cell 7:1180-90.

111. Znaidi S, Weber S, Al-Abdin OZ, Bomme P, Saidane S, Drouin S, Lemieux S, De Deken X, Robert F, Raymond M. 2008. Genomewide location analysis of Candida albicans Upc2p, a regulator of sterol metabolism and azole drug resistance. Eukaryot Cell 7:836-47.

112. Schubert S, Barker KS, Znaidi S, Schneider S, Dierolf F, Dunkel N, Aid M, Boucher G, Rogers PD, Raymond M, Morschhauser J. 2011. Regulation of efflux pump expression and drug resistance by the transcription factors Mrr1, Upc2, and Cap1 in Candida albicans. Antimicrob Agents Chemother 55:2212-23. 
113. Heilmann CJ, Schneider S, Barker KS, Rogers PD, Morschhauser J. 2010. An A643T mutation in the transcription factor Upc2p causes constitutive ERG1 1 upregulation and increased fluconazole resistance in Candida albicans. Antimicrob Agents Chemother 54:353-9.

114. Hoot SJ, Smith AR, Brown RP, White TC. 2011. An A643V amino acid substitution in Upc2p contributes to azole resistance in well-characterized clinical isolates of Candida albicans. Antimicrob Agents Chemother 55:940-2.

115. Flowers SA, Barker KS, Berkow EL, Toner G, Chadwick SG, Gygax SE, Morschhauser J, Rogers PD. 2012. Gain-of-function mutations in UPC2 are a frequent cause of ERG11 upregulation in azole-resistant clinical isolates of Candida albicans. Eukaryot Cell 11:1289-99.

116. Vasicek EM, Berkow EL, Flowers SA, Barker KS, Rogers PD. 2014. UPC2 is universally essential for azole antifungal resistance in Candida albicans. Eukaryot Cell 13:933-46.

117. Schillig R, Morschhauser J. 2013. Analysis of a fungus-specific transcription factor family, the Candida albicans zinc cluster proteins, by artificial activation. Mol Microbiol 89:1003-17.

118. Wang Y, Liu JY, Shi C, Li WJ, Zhao Y, Yan L, Xiang MJ. 2015. Mutations in transcription factor Mrr2p contribute to fluconazole resistance in clinical isolates of Candida albicans. Int J Antimicrob Agents 46:552-9.

119. Nishimoto AT, Zhang Q, Hazlett B, Morschhäuser J, Rogers PD. 2019. The contribution of clinically-derived mutations in the gene encoding the zinc cluster transcription factor Mrr2 to fluconazole antifungal resistance and $C D R 1$ expression in Candida albicans. Antimicrobial Agents and Chemotherapy doi:10.1128/aac.00078-19:AAC.00078-19.

120. Sanguinetti M, Posteraro B, Fiori B, Ranno S, Torelli R, Fadda G. 2005. Mechanisms of azole resistance in clinical isolates of Candida glabrata collected during a hospital survey of antifungal resistance. Antimicrob Agents Chemother 49:668-79.

121. White TC. 1997. The presence of an R467K amino acid substitution and loss of allelic variation correlate with an azole-resistant lanosterol 14alpha demethylase in Candida albicans. Antimicrob Agents Chemother 41:1488-94.

122. Sanglard D, Ischer F, Koymans L, Bille J. 1998. Amino acid substitutions in the cytochrome P-450 lanosterol 14alpha-demethylase (CYP51A1) from azoleresistant Candida albicans clinical isolates contribute to resistance to azole antifungal agents. Antimicrob Agents Chemother 42:241-53. 
123. Kelly SL, Lamb DC, Kelly DE. 1999. Y132H substitution in Candida albicans sterol 14alpha-demethylase confers fluconazole resistance by preventing binding to haem. FEMS Microbiol Lett 180:171-5.

124. Kelly SL, Lamb DC, Loeffler J, Einsele H, Kelly DE. 1999. The G464S amino acid substitution in Candida albicans sterol 14alpha-demethylase causes fluconazole resistance in the clinic through reduced affinity. Biochem Biophys Res Commun 262:174-9.

125. Wang YB, Wang H, Guo HY, Zhao YZ, Luo SQ. 2005. [Analysis of ERG11 gene mutation in Candida albicans]. Di Yi Jun Yi Da Xue Xue Bao 25:1390-3.

126. Xu Y, Chen L, Li C. 2008. Susceptibility of clinical isolates of Candida species to fluconazole and detection of Candida albicans ERG11 mutations. J Antimicrob Chemother 61:798-804.

127. Wang H, Kong F, Sorrell TC, Wang B, McNicholas P, Pantarat N, Ellis D, Xiao M, Widmer F, Chen SC. 2009. Rapid detection of ERG11 gene mutations in clinical Candida albicans isolates with reduced susceptibility to fluconazole by rolling circle amplification and DNA sequencing. BMC Microbiol 9:167.

128. Feng LJ, Wan Z, Wang XH, Li RY, Liu W. 2010. Relationship between antifungal resistance of fluconazole resistant Candida albicans and mutations in ERG11 gene. Chin Med J (Engl) 123:544-8.

129. Kim TH, Lee MK. 2010. Evaluation of the V404I and V509M amino acid substitutions of ERG11 gene in Candida albicans isolates by pyrosequencing. Folia Microbiol (Praha) 55:301-4.

130. Martel CM, Parker JE, Bader O, Weig M, Gross U, Warrilow AG, Kelly DE, Kelly SL. 2010. A clinical isolate of Candida albicans with mutations in ERG11 (encoding sterol 14alpha-demethylase) and ERG5 (encoding C22 desaturase) is cross resistant to azoles and amphotericin B. Antimicrob Agents Chemother 54:3578-83.

131. Morio F, Loge C, Besse B, Hennequin C, Le Pape P. 2010. Screening for amino acid substitutions in the Candida albicans Erg11 protein of azole-susceptible and azole-resistant clinical isolates: new substitutions and a review of the literature. Diagn Microbiol Infect Dis 66:373-84.

132. Oliveira Carvalho V, Okay TS, Melhem MS, Walderez Szeszs M, del Negro GM. 2013. The new mutation L321F in Candida albicans ERG11 gene may be associated with fluconazole resistance. Rev Iberoam Micol 30:209-12. 
133. Strzelczyk JK, Slemp-Migiel A, Rother M, Golabek K, Wiczkowski A. 2013. Nucleotide substitutions in the Candida albicans ERG11 gene of azole-susceptible and azole-resistant clinical isolates. Acta Biochim Pol 60:547-52.

134. Xiang MJ, Liu JY, Ni PH, Wang S, Shi C, Wei B, Ni YX, Ge HL. 2013. Erg11 mutations associated with azole resistance in clinical isolates of Candida albicans. FEMS Yeast Res 13:386-93.

135. Ying Y, Zhao Y, Hu X, Cai Z, Liu X, Jin G, Zhang J, Zhang J, Liu J, Huang X. 2013. In vitro fluconazole susceptibility of 1,903 clinical isolates of Candida albicans and the identification of ERG11 mutations. Microb Drug Resist 19:26673.

136. Zhang L, Yang HF, Liu YY, Xu XH, Ye Y, Li JB. 2013. Reduced susceptibility of Candida albicans clinical isolates to azoles and detection of mutations in the ERG11 gene. Diagn Microbiol Infect Dis 77:327-9.

137. Zhao J, Xu Y, Li C. 2013. Association of T916C (Y257H) mutation in Candida albicans ERG11 with fluconazole resistance. Mycoses 56:315-20.

138. Oliveira-Carvalho V, Del Negro GM. 2014. Is the S405F mutation in Candida albicans ERG11 gene sufficient to confer resistance to fluconazole? J Mycol Med 24:241-2.

139. Flowers SA, Colon B, Whaley SG, Schuler MA, Rogers PD. 2015. Contribution of clinically derived mutations in ERG11 to azole resistance in Candida albicans. Antimicrob Agents Chemother 59:450-60.

140. Hargrove TY, Friggeri L, Wawrzak Z, Qi A, Hoekstra WJ, Schotzinger RJ, York JD, Guengerich FP, Lepesheva GI. 2017. Structural analyses of Candida albicans sterol 14alpha-demethylase complexed with azole drugs address the molecular basis of azole-mediated inhibition of fungal sterol biosynthesis. J Biol Chem 292:6728-6743.

141. Warrilow AG, Nishimoto AT, Parker JE, Price CL, Flowers SA, Kelly DE, Rogers PD, Kelly SL. 2019. The Evolution of Azole Resistance in Candida albicans Sterol 14alpha-Demethylase (CYP51) through Incremental Amino Acid Substitutions. Antimicrob Agents Chemother doi:10.1128/AAC.02586-18.

142. Martel CM, Parker JE, Bader O, Weig M, Gross U, Warrilow AG, Rolley N, Kelly DE, Kelly SL. 2010. Identification and characterization of four azoleresistant erg3 mutants of Candida albicans. Antimicrob Agents Chemother 54:4527-33. 
143. Miyazaki Y, Geber A, Miyazaki H, Falconer D, Parkinson T, Hitchcock C, Grimberg B, Nyswaner K, Bennett JE. 1999. Cloning, sequencing, expression and allelic sequence diversity of ERG3 (C-5 sterol desaturase gene) in Candida albicans. Gene 236:43-51.

144. Howell SA, Mallet AI, Noble WC. 1990. A comparison of the sterol content of multiple isolates of the Candida albicans Darlington strain with other clinically azole-sensitive and -resistant strains. J Appl Bacteriol 69:692-6.

145. Morio F, Pagniez F, Lacroix C, Miegeville M, Le Pape P. 2012. Amino acid substitutions in the Candida albicans sterol Delta5,6-desaturase (Erg3p) confer azole resistance: characterization of two novel mutants with impaired virulence. J Antimicrob Chemother 67:2131-8.

146. Miyazaki T, Miyazaki Y, Izumikawa K, Kakeya H, Miyakoshi S, Bennett JE, Kohno S. 2006. Fluconazole treatment is effective against a Candida albicans erg3/erg3 mutant in vivo despite in vitro resistance. Antimicrob Agents Chemother 50:580-6.

147. Vale-Silva LA, Coste AT, Ischer F, Parker JE, Kelly SL, Pinto E, Sanglard D. 2012. Azole resistance by loss of function of the sterol Delta(5),(6)-desaturase gene (ERG3) in Candida albicans does not necessarily decrease virulence. Antimicrob Agents Chemother 56:1960-8.

148. Chau AS, Gurnani M, Hawkinson R, Laverdiere M, Cacciapuoti A, McNicholas PM. 2005. Inactivation of sterol Delta5,6-desaturase attenuates virulence in Candida albicans. Antimicrob Agents Chemother 49:3646-51.

149. Luna-Tapia A, Willems HME, Parker JE, Tournu H, Barker KS, Nishimoto AT, Rogers PD, Kelly SL, Peters BM, Palmer GE. 2018. Loss of Upc2p-Inducible ERG3 Transcription Is Sufficient To Confer Niche-Specific Azole Resistance without Compromising Candida albicans Pathogenicity. MBio 9.

150. Perepnikhatka V, Fischer FJ, Niimi M, Baker RA, Cannon RD, Wang YK, Sherman F, Rustchenko E. 1999. Specific chromosome alterations in fluconazoleresistant mutants of Candida albicans. J Bacteriol 181:4041-9.

151. Chen X, Magee BB, Dawson D, Magee PT, Kumamoto CA. 2004. Chromosome 1 trisomy compromises the virulence of Candida albicans. Mol Microbiol 51:55165.

152. Cowen LE, Sanglard D, Calabrese D, Sirjusingh C, Anderson JB, Kohn LM. 2000. Evolution of drug resistance in experimental populations of Candida albicans. J Bacteriol 182:1515-22. 
153. Forche A, May G, Magee PT. 2005. Demonstration of loss of heterozygosity by single-nucleotide polymorphism microarray analysis and alterations in strain morphology in Candida albicans strains during infection. Eukaryot Cell 4:156-65.

154. Selmecki A, Bergmann S, Berman J. 2005. Comparative genome hybridization reveals widespread aneuploidy in Candida albicans laboratory strains. Mol Microbiol 55:1553-65.

155. Selmecki A, Forche A, Berman J. 2006. Aneuploidy and isochromosome formation in drug-resistant Candida albicans. Science 313:367-70.

156. Ford CB, Funt JM, Abbey D, Issi L, Guiducci C, Martinez DA, Delorey T, Li BY, White TC, Cuomo C, Rao RP, Berman J, Thompson DA, Regev A. 2015. The evolution of drug resistance in clinical isolates of Candida albicans. Elife 4:e0662.

157. Legrand M, Lephart P, Forche A, Mueller FM, Walsh T, Magee PT, Magee BB. 2004. Homozygosity at the MTL locus in clinical strains of Candida albicans: karyotypic rearrangements and tetraploid formation. Mol Microbiol 52:1451-62.

158. Hickman MA, Paulson C, Dudley A, Berman J. 2015. Parasexual Ploidy Reduction Drives Population Heterogeneity Through Random and Transient Aneuploidy in Candida albicans. Genetics 200:781-94.

159. Li X, Yang F, Li D, Zhou M, Wang X, Xu Q, Zhang Y, Yan L, Jiang Y. 2015. Trisomy of chromosome $\mathrm{R}$ confers resistance to triazoles in Candida albicans. Med Mycol 53:302-9.

160. Anderson MZ, Saha A, Haseeb A, Bennett RJ. 2017. A chromosome 4 trisomy contributes to increased fluconazole resistance in a clinical isolate of Candida albicans. Microbiology 163:856-865.

161. Mansfield BE, Oltean HN, Oliver BG, Hoot SJ, Leyde SE, Hedstrom L, White TC. 2010. Azole drugs are imported by facilitated diffusion in Candida albicans and other pathogenic fungi. PLoS Pathog 6:e1001126.

162. Esquivel BD, Smith AR, Zavrel M, White TC. 2015. Azole drug import into the pathogenic fungus Aspergillus fumigatus. Antimicrob Agents Chemother 59:3390-8.

163. Pfaller MA, Andes D, Arendrup MC, Diekema DJ, Espinel-Ingroff A, Alexander BD, Brown SD, Chaturvedi V, Fowler CL, Ghannoum MA, Johnson EM, Knapp CC, Motyl MR, Ostrosky-Zeichner L, Walsh TJ. 2011. Clinical breakpoints for voriconazole and Candida spp. revisited: review of microbiologic, molecular, pharmacodynamic, and clinical data as they pertain to the development of speciesspecific interpretive criteria. Diagn Microbiol Infect Dis 70:330-43. 
164. Pfaller MA, Castanheira M, Diekema DJ, Messer SA, Jones RN. 2011. Triazole and echinocandin MIC distributions with epidemiological cutoff values for differentiation of wild-type strains from non-wild-type strains of six uncommon species of Candida. J Clin Microbiol 49:3800-4.

165. European Committee on Antimicrobial Susceptibility Testing. 2018. Antifungal Agents: Breakpoint tables for interpretation of MICs.

166. Fridman O, Goldberg A, Ronin I, Shoresh N, Balaban NQ. 2014. Optimization of lag time underlies antibiotic tolerance in evolved bacterial populations. Nature 513:418-21.

167. Brauner A, Fridman O, Gefen O, Balaban NQ. 2016. Distinguishing between resistance, tolerance and persistence to antibiotic treatment. Nat Rev Microbiol 14:320-30.

168. Delarze E, Sanglard D. 2015. Defining the frontiers between antifungal resistance, tolerance and the concept of persistence. Drug Resist Updat 23:12-19.

169. Marchetti O, Moreillon P, Glauser MP, Bille J, Sanglard D. 2000. Potent synergism of the combination of fluconazole and cyclosporine in Candida albicans. Antimicrob Agents Chemother 44:2373-81.

170. Arthington-Skaggs BA, Lee-Yang W, Ciblak MA, Frade JP, Brandt ME, Hajjeh RA, Harrison LH, Sofair AN, Warnock DW, Candidemia Active Surveillance G. 2002. Comparison of visual and spectrophotometric methods of broth microdilution MIC end point determination and evaluation of a sterol quantitation method for in vitro susceptibility testing of fluconazole and itraconazole against trailing and nontrailing Candida isolates. Antimicrob Agents Chemother 46:247781 .

171. Lee MK, Williams LE, Warnock DW, Arthington-Skaggs BA. 2004. Drug resistance genes and trailing growth in Candida albicans isolates. J Antimicrob Chemother 53:217-24.

172. Whaley SG, Tsao S, Weber S, Zhang Q, Barker KS, Raymond M, Rogers PD. 2016. The RTA3 Gene, Encoding a Putative Lipid Translocase, Influences the Susceptibility of Candida albicans to Fluconazole. Antimicrob Agents Chemother 60:6060-6.

173. Luna-Tapia A, Butts A, Palmer GE. 2019. Loss of C-5 Sterol Desaturase Activity in Candida albicans: Azole Resistance or Merely Trailing Growth? Antimicrob Agents Chemother 63. 
174. Marr KA, Rustad TR, Rex JH, White TC. 1999. The trailing end point phenotype in antifungal susceptibility testing is $\mathrm{pH}$ dependent. Antimicrob Agents Chemother 43:1383-6.

175. Revankar SG, Kirkpatrick WR, McAtee RK, Fothergill AW, Redding SW, Rinaldi MG, Patterson TF. 1998. Interpretation of trailing endpoints in antifungal susceptibility testing by the National Committee for Clinical Laboratory Standards method. J Clin Microbiol 36:153-6.

176. Rex JH, Nelson PW, Paetznick VL, Lozano-Chiu M, Espinel-Ingroff A, Anaissie EJ. 1998. Optimizing the correlation between results of testing in vitro and therapeutic outcome in vivo for fluconazole by testing critical isolates in a murine model of invasive candidiasis. Antimicrob Agents Chemother 42:129-34.

177. Arthington-Skaggs BA, Warnock DW, Morrison CJ. 2000. Quantitation of Candida albicans ergosterol content improves the correlation between in vitro antifungal susceptibility test results and in vivo outcome after fluconazole treatment in a murine model of invasive candidiasis. Antimicrob Agents Chemother 44:2081-5.

178. Rosenberg A, Ene IV, Bibi M, Zakin S, Segal ES, Ziv N, Dahan AM, Colombo AL, Bennett RJ, Berman J. 2018. Antifungal tolerance is a subpopulation effect distinct from resistance and is associated with persistent candidemia. Nat Commun 9:2470.

179. Gao J, Wang H, Li Z, Wong AH, Wang YZ, Guo Y, Lin X, Zeng G, Liu H, Wang Y, Wang J. 2018. Candida albicans gains azole resistance by altering sphingolipid composition. Nat Commun 9:4495.

180. McCarty TP, Pappas PG. 2016. Invasive Candidiasis. Infect Dis Clin North Am 30:103-24.

181. Kullberg BJ, Arendrup MC. 2015. Invasive Candidiasis. N Engl J Med 373:144556.

182. Morgan J, Meltzer MI, Plikaytis BD, Sofair AN, Huie-White S, Wilcox S, Harrison LH, Seaberg EC, Hajjeh RA, Teutsch SM. 2005. Excess mortality, hospital stay, and cost due to candidemia: a case-control study using data from population-based candidemia surveillance. Infect Control Hosp Epidemiol 26:540-7.

183. Magill SS, Edwards JR, Bamberg W, Beldavs ZG, Dumyati G, Kainer MA, Lynfield R, Maloney M, McAllister-Hollod L, Nadle J, Ray SM, Thompson DL, Wilson LE, Fridkin SK, Emerging Infections Program Healthcare-Associated I, Antimicrobial Use Prevalence Survey T. 2014. Multistate point-prevalence survey of health care-associated infections. N Engl J Med 370:1198-208. 
184. Centers for Disease Control and Prevention. 2013. Antibiotic Resistance Threats in the United States. https://www.cdc.gov/drugresistance/threat-report-2013/pdf/. https://www.cdc.gov/drugresistance/threat-report-2013/pdf/.

185. Sangeorzan JA, Bradley SF, He X, Zarins LT, Ridenour GL, Tiballi RN, Kauffman CA. 1994. Epidemiology of oral candidiasis in HIV-infected patients: colonization, infection, treatment, and emergence of fluconazole resistance. Am J Med 97:339-46.

186. Shah DN, Yau R, Lasco TM, Weston J, Salazar M, Palmer HR, Garey KW. 2012. Impact of prior inappropriate fluconazole dosing on isolation of fluconazolenonsusceptible Candida species in hospitalized patients with candidemia. Antimicrob Agents Chemother 56:3239-43.

187. Break TJ, Desai JV, Healey KR, Natarajan M, Ferre EMN, Henderson C, Zelazny A, Siebenlist U, Yates CM, Cohen OJ, Schotzinger RJ, Perlin DS, Garvey EP, Lionakis MS. 2018. VT-1598 inhibits the in vitro growth of mucosal Candida strains and protects against fluconazole-susceptible and -resistant oral candidiasis in IL-17 signalling-deficient mice. J Antimicrob Chemother 73:2089-2094.

188. Warrilow AG, Hull CM, Parker JE, Garvey EP, Hoekstra WJ, Moore WR, Schotzinger RJ, Kelly DE, Kelly SL. 2014. The clinical candidate VT-1161 is a highly potent inhibitor of Candida albicans CYP51 but fails to bind the human enzyme. Antimicrob Agents Chemother 58:7121-7.

189. Yates CM, Garvey EP, Shaver SR, Schotzinger RJ, Hoekstra WJ. 2017. Design and optimization of highly-selective, broad spectrum fungal CYP51 inhibitors. Bioorg Med Chem Lett 27:3243-3248.

190. Pfaller M, Neofytos D, Diekema D, Azie N, Meier-Kriesche HU, Quan SP, Horn D. 2012. Epidemiology and outcomes of candidemia in 3648 patients: data from the Prospective Antifungal Therapy (PATH Alliance(R)) registry, 2004-2008. Diagn Microbiol Infect Dis 74:323-31.

191. Goldman M, Cloud GA, Wade KD, Reboli AC, Fichtenbaum CJ, Hafner R, Sobel JD, Powderly WG, Patterson TF, Wheat LJ, Stein DK, Dismukes WE, Filler SG, Team ACTGS, Mycoses Study Group Study T. 2005. A randomized study of the use of fluconazole in continuous versus episodic therapy in patients with advanced HIV infection and a history of oropharyngeal candidiasis: AIDS Clinical Trials Group Study 323/Mycoses Study Group Study 40. Clin Infect Dis 41:1473-80.

192. Hajjeh RA, Sofair AN, Harrison LH, Lyon GM, Arthington-Skaggs BA, Mirza SA, Phelan M, Morgan J, Lee-Yang W, Ciblak MA, Benjamin LE, Sanza LT, Huie S, Yeo SF, Brandt ME, Warnock DW. 2004. Incidence of bloodstream infections due to Candida species and in vitro susceptibilities of isolates collected 
from 1998 to 2000 in a population-based active surveillance program. J Clin Microbiol 42:1519-27.

193. Klevay MJ, Ernst EJ, Hollanbaugh JL, Miller JG, Pfaller MA, Diekema DJ. 2008. Therapy and outcome of Candida glabrata versus Candida albicans bloodstream infection. Diagn Microbiol Infect Dis 60:273-7.

194. Moriyama B, Gordon LA, McCarthy M, Henning SA, Walsh TJ, Penzak SR. 2014. Emerging drugs and vaccines for candidemia. Mycoses 57:718-33.

195. MacPherson S, Larochelle M, Turcotte B. 2006. A fungal family of transcriptional regulators: the zinc cluster proteins. Microbiol Mol Biol Rev 70:583-604.

196. Tsao S, Rahkhoodaee F, Raymond M. 2009. Relative contributions of the Candida albicans $\mathrm{ABC}$ transporters Cdrlp and Cdr2p to clinical azole resistance. Antimicrob Agents Chemother 53:1344-52.

197. Hampe IAI, Friedman J, Edgerton M, Morschhauser J. 2017. An acquired mechanism of antifungal drug resistance simultaneously enables Candida albicans to escape from intrinsic host defenses. PLoS Pathog 13:e1006655.

198. Vargas-Blanco D, Lynn A, Rosch J, Noreldin R, Salerni A, Lambert C, Rao RP. 2017. A pre-therapeutic coating for medical devices that prevents the attachment of Candida albicans. Ann Clin Microbiol Antimicrob 16:41.

199. Partow S, Siewers V, Bjorn S, Nielsen J, Maury J. 2010. Characterization of different promoters for designing a new expression vector in Saccharomyces cerevisiae. Yeast 27:955-64.

200. Clinical and Laboratory Standards Institute. 2008. Reference method for broth microdilution antifungal susceptibility testing of yeasts; approved standard, 3rd ed. CLSI document M27-A3, Wayne, PA.

201. Clinical and Laboratory Standards Institute. 2012. Reference method for broth microdilution antifungal susceptibility testing of yeasts; fourth informational supplement. CLSI document M27-S4, Wayne, PA.

202. Reuss O, Vik A, Kolter R, Morschhauser J. 2004. The SAT1 flipper, an optimized tool for gene disruption in Candida albicans. Gene 341:119-27.

203. Grahl N, Demers EG, Crocker AW, Hogan DA. 2017. Use of RNA-Protein Complexes for Genome Editing in Non-albicans Candida Species. mSphere 2.

204. Kao AS, Brandt ME, Pruitt WR, Conn LA, Perkins BA, Stephens DS, Baughman WS, Reingold AL, Rothrock GA, Pfaller MA, Pinner RW, Hajjeh RA. 1999. The 
epidemiology of candidemia in two United States cities: results of a populationbased active surveillance. Clin Infect Dis 29:1164-70.

205. Marichal P, Koymans L, Willemsens S, Bellens D, Verhasselt P, Luyten W, Borgers M, Ramaekers FCS, Odds FC, Vanden Bossche H. 1999. Contribution of mutations in the cytochrome P450 14alpha-demethylase (Erg11p, Cyp51p) to azole resistance in Candida albicans. Microbiology 145 ( Pt 10):2701-2713.

206. Keniya MV, Sabherwal M, Wilson RK, Woods MA, Sagatova AA, Tyndall JDA, Monk BC. 2018. Crystal Structures of Full-Length Lanosterol 14alphaDemethylases of Prominent Fungal Pathogens Candida albicans and Candida glabrata Provide Tools for Antifungal Discovery. Antimicrob Agents Chemother 62.

207. Popp C, Hampe IAI, Hertlein T, Ohlsen K, Rogers PD, Morschhauser J. 2017. Competitive Fitness of Fluconazole-Resistant Clinical Candida albicans Strains. Antimicrob Agents Chemother 61.

208. Sasse C, Dunkel N, Schafer T, Schneider S, Dierolf F, Ohlsen K, Morschhauser J. 2012. The stepwise acquisition of fluconazole resistance mutations causes a gradual loss of fitness in Candida albicans. Mol Microbiol 86:539-56.

209. Lohberger A, Coste AT, Sanglard D. 2014. Distinct roles of Candida albicans drug resistance transcription factors TAC1, MRR1, and UPC2 in virulence. Eukaryot Cell 13:127-42.

210. Morschhäuser J. 2019. Competition experiments.

211. Sobel JD. 2007. Vulvovaginal candidosis. Lancet 369:1961-71.

212. Buchacz K, Lau B, Jing Y, Bosch R, Abraham AG, Gill MJ, Silverberg MJ, Goedert JJ, Sterling TR, Althoff KN, Martin JN, Burkholder G, Gandhi N, Samji H, Patel P, Rachlis A, Thorne JE, Napravnik S, Henry K, Mayor A, Gebo K, Gange SJ, Moore RD, Brooks JT, North American ACCoR, Design of Ie DEA. 2016. Incidence of AIDS-Defining Opportunistic Infections in a Multicohort Analysis of HIV-infected Persons in the United States and Canada, 2000-2010. J Infect Dis 214:862-72.

213. Coronado-Castellote L, Jimenez-Soriano Y. 2013. Clinical and microbiological diagnosis of oral candidiasis. J Clin Exp Dent 5:e279-86.

214. Song JL, Harry JB, Eastman RT, Oliver BG, White TC. 2004. The Candida albicans lanosterol 14-alpha-demethylase (ERG11) gene promoter is maximally induced after prolonged growth with antifungal drugs. Antimicrob Agents Chemother 48:1136-44. 
215. Ribeiro MA, Paula CR. 2007. Up-regulation of ERG11 gene among fluconazoleresistant Candida albicans generated in vitro: is there any clinical implication? Diagn Microbiol Infect Dis 57:71-5.

216. Arthington-Skaggs BA, Crowell DN, Yang H, Sturley SL, Bard M. 1996. Positive and negative regulation of a sterol biosynthetic gene (ERG3) in the post-squalene portion of the yeast ergosterol pathway. FEBS Lett 392:161-5.

217. Vasicek EM, Berkow EL, Bruno VM, Mitchell AP, Wiederhold NP, Barker KS, Rogers PD. 2014. Disruption of the transcriptional regulator Cas5 results in enhanced killing of Candida albicans by Fluconazole. Antimicrob Agents Chemother 58:6807-18.

218. Berberi A, Noujeim Z, Aoun G. 2015. Epidemiology of Oropharyngeal Candidiasis in Human Immunodeficiency Virus/Acquired Immune Deficiency Syndrome Patients and CD4+ Counts. J Int Oral Health 7:20-3.

219. Solomon SL, Oliver KB. 2014. Antibiotic resistance threats in the United States: stepping back from the brink. Am Fam Physician 89:938-41.

220. Rex JH, Rinaldi MG, Pfaller MA. 1995. Resistance of Candida species to fluconazole. Antimicrob Agents Chemother 39:1-8.

221. Ruhnke M, Eigler A, Tennagen I, Geiseler B, Engelmann E, Trautmann M. 1994. Emergence of fluconazole-resistant strains of Candida albicans in patients with recurrent oropharyngeal candidosis and human immunodeficiency virus infection. J Clin Microbiol 32:2092-8.

222. Bhattacharya S, Sobel JD, White TC. 2016. A Combination Fluorescence Assay Demonstrates Increased Efflux Pump Activity as a Resistance Mechanism in Azole-Resistant Vaginal Candida albicans Isolates. Antimicrob Agents Chemother 60:5858-66.

223. Kontoyiannis DP. 2017. Antifungal Resistance: An Emerging Reality and A Global Challenge. J Infect Dis 216:S431-S435.

224. Kelly SL, Lamb DC, Corran AJ, Baldwin BC, Kelly DE. 1995. Mode of action and resistance to azole antifungals associated with the formation of 14 alphamethylergosta-8,24(28)-dien-3 beta,6 alpha-diol. Biochem Biophys Res Commun 207:910-5.

225. Hoekstra WJ, Garvey EP, Moore WR, Rafferty SW, Yates CM, Schotzinger RJ. 2014. Design and optimization of highly-selective fungal CYP51 inhibitors. Bioorg Med Chem Lett 24:3455-8. 
226. Wiederhold NP, Patterson HP, Tran BH, Yates CM, Schotzinger RJ, Garvey EP. 2018. Fungal-specific Cyp51 inhibitor VT-1598 demonstrates in vitro activity against Candida and Cryptococcus species, endemic fungi, including Coccidioides species, Aspergillus species and Rhizopus arrhizus. J Antimicrob Chemother $73: 404-408$.

227. Wiederhold NP, Shubitz LF, Najvar LK, Jaramillo R, Olivo M, Catano G, Trinh HT, Yates CM, Schotzinger RJ, Garvey EP, Patterson TF. 2018. The Novel Fungal Cyp51 Inhibitor VT-1598 Is Efficacious in Experimental Models of Central Nervous System Coccidioidomycosis Caused by Coccidioides posadasii and Coccidioides immitis. Antimicrob Agents Chemother 62.

228. Garvey EP, Sharp AD, Warn PA, Yates CM, Schotzinger RJ. 2018. The novel fungal CYP51 inhibitor VT-1598 is efficacious alone and in combination with liposomal amphotericin B in a murine model of cryptococcal meningitis. J Antimicrob Chemother 73:2815-2822.

229. Hargrove TY, Garvey EP, Hoekstra WJ, Yates CM, Wawrzak Z, Rachakonda G, Villalta F, Lepesheva GI. 2017. Crystal Structure of the New Investigational Drug Candidate VT-1598 in Complex with Aspergillus fumigatus Sterol 14alphaDemethylase Provides Insights into Its Broad-Spectrum Antifungal Activity. Antimicrob Agents Chemother 61.

230. Monk BC, Keniya MV, Sabherwal M, Wilson RK, Graham DO, Hassan HF, Chen D, Tyndall JDA. 2019. Azole Resistance Reduces Susceptibility to the Tetrazole Antifungal VT-1161. Antimicrob Agents Chemother 63.

231. Hoot SJ, Brown RP, Oliver BG, White TC. 2010. The UPC2 promoter in Candida albicans contains two cis-acting elements that bind directly to Upc2p, resulting in transcriptional autoregulation. Eukaryot Cell 9:1354-62. 


\section{APPENDIX A. AMINO ACID SUBSTITUTIONS IN MRR2 CLINICAL C. ALBICANS ISOLATES}

Table A-1. Amino acid substitutions in Mrr2 clinical $C$. albicans isolates

\begin{tabular}{cl}
\hline Isolate & \multicolumn{1}{c}{ Amino Acid Substitutions ${ }^{\mathrm{a}, \mathrm{b}}$} \\
\hline FLU-susceptible & V451A (h) \\
1 & - \\
2 & S143P, L144V, T145A, S165N, V451A, S480P \\
3 & - \\
5 & - \\
7 & V451A \\
9 & V451A \\
10 & - \\
17 & T83A, V451A, V582L \\
22 & -
\end{tabular}

FLU-resistant

11

12

13

14

15

16

18

19

20

21

23

24

25

26

27

28

29

30

31

32

33

34

35

36
V451A, S480P

V451A

$-$

T83A, A459T, S480P, V485M

S466L, A468G, S469T, T470N, S480P

S143P, L144V, T145A, S165N, A459T, S480P

S143P, L144V, T145A, S165N, A459T, S480P

S143P, L144V, T145A, S165N, A459T, S480P

V451A

R45Q, V451A

S143P (h), L144V (h), T145A (h), V451A (h), S466L, A468G, S469T, T470N, S480P

T83A, A459T, S480P, V486M

T83A, A459T, S480P, V486M

S466L, A468G, S469T, T470N, S480P

$-$

$-$

S143P, L144V, T145A, S165N, A459T, S480P

$-$

- 
Table A-1. $\quad$ (Continued)

\begin{tabular}{|c|c|}
\hline Isolate & Amino Acid Substitutions ${ }^{\mathrm{a}, \mathrm{b}}$ \\
\hline \multicolumn{2}{|c|}{ FLU-resistant } \\
\hline 37 & - \\
\hline 38 & - \\
\hline 39 & - \\
\hline 40 & V451A \\
\hline 41 & S143P, L144V, T145A, S165N, A459T, S480P \\
\hline 42 & - \\
\hline 43 & - \\
\hline 44 & - \\
\hline 45 & T83A, T145A, V451A, S480P \\
\hline 46 & $\begin{array}{l}\text { S143P (h), L144 (h), T145A (h), V451A (h), S466L, A468G, S469T, T470N, } \\
\text { S480P }\end{array}$ \\
\hline 47 & T83A, V451A, S480P \\
\hline 48 & S143P, L144V, T145A, S165N, A459T, S480P \\
\hline 49 & - \\
\hline 50 & - \\
\hline 51 & - \\
\hline 54 & S143P, L144V, T145A, S165N, A459T, S480P \\
\hline 55 & - \\
\hline 56 & - \\
\hline 58 & - \\
\hline 59 & T83A, L143P, L144V, T145A, S165N, V451A \\
\hline 60 & - \\
\hline 61 & - \\
\hline 62 & - \\
\hline 63 & - \\
\hline 64 & - \\
\hline 65 & - \\
\hline 66 & - \\
\hline 67 & - \\
\hline 68 & S143P (h), L144V (h), T145A (h), S165N (h), S480P (h) \\
\hline 69 & - \\
\hline 70 & T83A (h), S143P (h), L144V (h), T145A (h), S165N (h), V451A, S480P (h) \\
\hline 71 & T83A, V451A, S480P \\
\hline 72 & - \\
\hline
\end{tabular}

${ }^{a}$ (h) indicates a heterozygous amino acid substitution

$\mathrm{b}$ "“_. Denotes no changes in the amino acid sequence of the clinical isolate from the $M R R 2$ allele of SC5314. 


\section{APPENDIX B. SUPPLEMENTARY DATA TABLES TO CHAPTER 5}

Table B-1. Minimum inhibitory concentration values for VT-1161, VT-1598, fluconazole, voriconazole, itraconazole, posaconazole, and known azole resistance mechanisms in clinical $C$. albicans isolates

\begin{tabular}{|c|c|c|c|c|c|c|c|c|c|c|c|c|c|c|}
\hline \multirow{2}{*}{$\begin{array}{l}\text { Isolate } \\
\text { ID }\end{array}$} & \multicolumn{2}{|c|}{ VT-1161 } & \multicolumn{2}{|c|}{ VT-1598 } & \multicolumn{2}{|c|}{ Fluconazole } & \multicolumn{2}{|c|}{ Voriconazole } & \multicolumn{2}{|c|}{ Itraconazole } & \multicolumn{2}{|c|}{ Posaconazole } & \multirow{2}{*}{$\begin{array}{c}\text { ERG11 } \\
\text { mutations }^{\text {a }}\end{array}$} & \multirow{2}{*}{$\begin{array}{c}\text { Other } \\
\text { Resistance } \\
\text { Mechanisms }^{\mathrm{b}}\end{array}$} \\
\hline & $\begin{array}{c}\text { MIC } \\
\# 1\end{array}$ & $\begin{array}{c}\mathrm{MIC} \\
\# 2\end{array}$ & $\begin{array}{c}\mathrm{MIC} \\
\# 1\end{array}$ & $\begin{array}{c}\mathrm{MIC} \\
\# 2\end{array}$ & $\begin{array}{c}\text { MIC } \\
\# 1\end{array}$ & $\begin{array}{c}\mathrm{MIC} \\
\# 2\end{array}$ & $\begin{array}{c}\mathrm{MIC} \\
\# 1\end{array}$ & $\begin{array}{c}\mathrm{MIC} \\
\# 2\end{array}$ & $\begin{array}{c}\text { MIC } \\
\# 1\end{array}$ & $\begin{array}{c}\mathrm{MIC} \\
\# 2\end{array}$ & $\begin{array}{c}\text { MIC } \\
\# 1\end{array}$ & $\begin{array}{c}\mathrm{MIC} \\
\# 2\end{array}$ & & \\
\hline 1 & $\leq 0.015$ & $\leq 0.015$ & $\leq 0.015$ & $\leq 0.015$ & 0.25 & 0.5 & $\leq 0.03$ & $\leq 0.03$ & 0.06 & $\leq 0.03$ & $\leq 0.03$ & 0.06 & $\begin{array}{l}\mathrm{K} 128 \mathrm{~T}^{(\mathrm{h})} \\
\mathrm{D} 116 \mathrm{E}^{(\mathrm{h})}\end{array}$ & --- \\
\hline 2 & $\leq 0.015$ & $\leq 0.015$ & $\leq 0.015$ & $\leq 0.015$ & $\leq 0.125$ & 0.25 & $\leq 0.03$ & $\leq 0.03$ & $\leq 0.03$ & 0.06 & $\leq 0.03$ & 0.06 & $\begin{array}{l}\text { F198fs }{ }^{(\mathrm{h})} \\
\text { K128T. D116E }\end{array}$ & --- \\
\hline 3 & $\leq 0.015$ & $\leq 0.015$ & $\leq 0.015$ & $\leq 0.015$ & 0.25 & 0.25 & $\leq 0.03$ & $\leq 0.03$ & $\leq 0.03$ & 0.06 & $\leq 0.03$ & 0.06 & G129A, D116E & --- \\
\hline 5 & $\leq 0.015$ & $\leq 0.015$ & $\leq 0.015$ & $\leq 0.015$ & 0.5 & 1 & $\leq 0.03$ & $\leq 0.03$ & NA & NA & NA & NA & NA & NA \\
\hline 6 & $\leq 0.015$ & $\leq 0.015$ & $\leq 0.015$ & $\leq 0.015$ & 1 & 1 & $\leq 0.03$ & $\leq 0.03$ & NA & NA & NA & NA & NA & NA \\
\hline 7 & $\leq 0.015$ & $\leq 0.015$ & $\leq 0.015$ & $\leq 0.015$ & $\leq 0.125$ & 0.25 & $\leq 0.03$ & $\leq 0.03$ & $\leq 0.03$ & $\leq 0.03$ & $\leq 0.03$ & 0.06 & $\begin{array}{l}\mathrm{K} 128 \mathrm{~T}^{(\mathrm{h})} \\
\mathrm{D} 116 \mathrm{E}^{(\mathrm{h})}\end{array}$ & --- \\
\hline 9 & $\leq 0.015$ & $\leq 0.015$ & $\leq 0.015$ & $\leq 0.015$ & $\leq 0.125$ & $\leq 0.125$ & $\leq 0.03$ & $\leq 0.03$ & $\leq 0.03$ & $\leq 0.03$ & $\leq 0.03$ & $\leq 0.03$ & $\begin{array}{l}\mathrm{S} 457 \mathrm{~F}^{(\mathrm{h})} \\
\mathrm{V} 437 \mathrm{I}^{(\mathrm{h})}\end{array}$ & --- \\
\hline 10 & $\leq 0.015$ & $\leq 0.015$ & $\leq 0.015$ & $\leq 0.015$ & $\leq 0.125$ & $\leq 0.125$ & $\leq 0.03$ & $\leq 0.03$ & $\leq 0.03$ & $\leq 0.03$ & $\leq 0.03$ & $\leq 0.03$ & V437I ${ }^{(h)}$ & --- \\
\hline 11 & 0.125 & 0.125 & 0.03 & $\leq 0.015$ & 8 & 4 & 0.25 & 0.25 & 0.5 & 0.5 & 0.25 & 0.5 & E266D, V488I & $\begin{array}{c}\uparrow E R G 11, \\
C D R 1\end{array}$ \\
\hline 12 & 0.125 & 0.125 & $\leq 0.015$ & $\leq 0.015$ & 32 & 32 & 0.06 & 0.125 & 0.25 & 0.125 & 0.125 & 0.25 & K143R & $\begin{array}{l}\uparrow E R G 11, \\
C D R 1\end{array}$ \\
\hline 13 & 0.06 & 0.06 & 0.03 & 0.06 & 32 & 32 & 0.125 & 0.25 & 0.25 & 0.5 & 0.25 & 0.5 & K143R & $\uparrow C D R 1$ \\
\hline 14 & 0.06 & 0.03 & $\leq 0.015$ & $\leq 0.015$ & 16 & 16 & 0.06 & 0.06 & 0.25 & 0.25 & 0.125 & 0.25 & K143R & $\uparrow C D R 1$ \\
\hline 15 & 0.125 & 0.06 & 0.06 & 0.03 & 16 & 16 & 0.25 & 0.25 & 0.5 & 0.5 & 0.25 & 0.5 & E266D, V488I & $\begin{array}{c}\uparrow E R G 11, \\
C D R 1\end{array}$ \\
\hline
\end{tabular}


Table B-1. (Continued)

\begin{tabular}{|c|c|c|c|c|c|c|c|c|c|c|c|c|c|c|}
\hline \multirow{2}{*}{$\begin{array}{l}\text { Isolate } \\
\text { ID }\end{array}$} & \multicolumn{2}{|c|}{ VT-1161 } & \multicolumn{2}{|c|}{ VT-1598 } & \multicolumn{2}{|c|}{ Fluconazole } & \multicolumn{2}{|c|}{ Voriconazole } & \multicolumn{2}{|c|}{ Itraconazole } & \multicolumn{2}{|c|}{ Posaconazole } & \multirow{2}{*}{$\begin{array}{c}\text { ERG11 } \\
\text { mutations }^{\mathrm{a}}\end{array}$} & \multirow{2}{*}{$\begin{array}{c}\text { Other } \\
\text { Resistance } \\
\text { Mechanisms }\end{array}$} \\
\hline & $\begin{array}{c}\text { MIC } \\
\# 1\end{array}$ & $\begin{array}{c}\text { MIC } \\
\# 2\end{array}$ & $\begin{array}{c}\text { MIC } \\
\# 1\end{array}$ & $\begin{array}{c}\text { MIC } \\
\# 2\end{array}$ & $\begin{array}{c}\text { MIC } \\
\# 1\end{array}$ & $\begin{array}{c}\text { MIC } \\
\# 2\end{array}$ & $\underset{\# 1}{\mathrm{MIC}}$ & $\begin{array}{c}\mathrm{MIC} \\
\# 2\end{array}$ & $\begin{array}{c}\text { MIC } \\
\# 1\end{array}$ & $\begin{array}{c}\text { MIC } \\
\# 2\end{array}$ & $\begin{array}{c}\text { MIC } \\
\# 1\end{array}$ & $\begin{array}{c}\mathrm{MIC} \\
\# 2\end{array}$ & & \\
\hline 16 & $\leq 0.015$ & 0.03 & $\leq 0.015$ & 0.03 & 4 & 8 & $\leq 0.03$ & 0.06 & 0.25 & 0.25 & 0.06 & 0.06 & $\mathrm{Q} 21 \mathrm{R}^{(\mathrm{h})}$ & $\uparrow M D R 1$ \\
\hline 17 & $\leq 0.015$ & $\leq 0.015$ & $\leq 0.015$ & $\leq 0.015$ & 2 & 2 & 0.06 & 0.5 & 0.125 & 0.25 & $\leq 0.03$ & 0.06 & A114S, Y257H & $\uparrow C D R 1$ \\
\hline 18 & 0.5 & 0.5 & 0.06 & 0.03 & 16 & 32 & 0.5 & 0.5 & 0.25 & 0.5 & 0.125 & 0.25 & S405F & $\begin{array}{c}\uparrow E R G 11, \\
C D R 1\end{array}$ \\
\hline 19 & 0.5 & 0.5 & $\leq 0.015$ & $\leq 0.015$ & 32 & 32 & 0.5 & 0.5 & 0.5 & 0.5 & 0.125 & 0.25 & S405F & $\begin{array}{c}\uparrow E R G 11, \\
C D R 1\end{array}$ \\
\hline 20 & 1 & 0.5 & 0.06 & 0.06 & 64 & 32 & 0.5 & 0.5 & 0.5 & 0.25 & 0.25 & 0.5 & $\mathrm{~S} 405 \mathrm{~F}$ & $\begin{array}{c}\uparrow E R G 11, \\
C D R 1\end{array}$ \\
\hline 21 & 1 & 0.5 & 0.125 & 0.06 & 32 & 32 & 0.5 & 0.5 & 0.5 & 0.5 & 0.25 & 0.5 & $\begin{array}{l}\text { A114V, } \\
\text { E266D, } \\
\text { H283R }\end{array}$ & $\begin{array}{c}\uparrow E R G 11, \\
C D R 1\end{array}$ \\
\hline 22 & $\leq 0.015$ & $\leq 0.015$ & $\leq 0.015$ & $\leq 0.015$ & 0.5 & 1 & $\leq 0.03$ & $\leq 0.03$ & $\leq 0.03$ & 0.06 & $\leq 0.03$ & 0.06 & F145L & --- \\
\hline 23 & 0.125 & 0.125 & 0.03 & $\leq 0.015$ & 32 & 32 & 0.5 & 0.25 & 0.125 & 0.25 & 0.06 & 0.125 & I483V, G450E & $\uparrow C D R 1$ \\
\hline 24 & 0.03 & 0.03 & 0.03 & 0.03 & 16 & 16 & 0.25 & 0.125 & 0.125 & 0.25 & 0.06 & 0.125 & G450E & $\begin{array}{l}\uparrow \mathrm{CDR} 1 \\
\mathrm{MDR} 1\end{array}$ \\
\hline 25 & $\leq 0.015$ & $\leq 0.015$ & $\leq 0.015$ & $\leq 0.015$ & 16 & 16 & 0.06 & 0.125 & 0.25 & 0.125 & $\leq 0.03$ & 0.125 & D446E & $\begin{array}{c}\uparrow E R G 11, \\
M D R 1\end{array}$ \\
\hline 26 & $\leq 0.015$ & $\leq 0.015$ & $\leq 0.015$ & $\leq 0.015$ & 16 & 16 & $\leq 0.03$ & $\leq 0.03$ & $\leq 0.03$ & $\leq 0.03$ & $\leq 0.03$ & $\leq 0.03$ & G307S , G448R & --- \\
\hline 27 & 0.06 & 0.06 & $\leq 0.015$ & 0.03 & 8 & 16 & 0.25 & 0.125 & 0.25 & 0.5 & 0.125 & 0.25 & E266D, V488I & $\begin{array}{c}\uparrow E R G 11, \\
C D R 1\end{array}$ \\
\hline 28 & 0.5 & 0.5 & $\leq 0.015$ & 0.06 & 64 & 64 & 0.5 & 1 & 0.5 & 0.5 & 0.25 & 1 & E266D, V488I & $\begin{array}{c}\uparrow E R G 11 \\
C D R 1\end{array}$ \\
\hline 29 & 0.25 & 0.5 & 0.06 & 0.06 & $>64$ & $>64$ & 2 & 2 & 0.5 & 0.5 & 0.25 & 1 & Y132F, F145L & $\uparrow C D R 1$ \\
\hline 30 & 0.25 & 0.5 & 0.06 & 0.06 & 16 & 32 & 0.5 & 1 & 0.06 & 0.125 & $\leq 0.03$ & $\leq 0.03$ & $\begin{array}{c}\text { F126L, Y132F, } \\
\text { H283R }\end{array}$ & $\uparrow E R G 11$ \\
\hline
\end{tabular}


Table B-1. (Continued)

\begin{tabular}{|c|c|c|c|c|c|c|c|c|c|c|c|c|c|c|}
\hline \multirow{2}{*}{$\begin{array}{l}\text { Isolate } \\
\text { ID }\end{array}$} & \multicolumn{2}{|c|}{ VT-1161 } & \multicolumn{2}{|c|}{ VT-1598 } & \multicolumn{2}{|c|}{ Fluconazole } & \multicolumn{2}{|c|}{ Voriconazole } & \multicolumn{2}{|c|}{ Itraconazole } & \multicolumn{2}{|c|}{ Posaconazole } & \multirow{2}{*}{$\begin{array}{c}\text { ERG11 } \\
\text { mutations }^{\text {a }}\end{array}$} & \multirow{2}{*}{$\begin{array}{c}\text { Other } \\
\text { Resistance } \\
\text { Mechanisms }\end{array}$} \\
\hline & $\begin{array}{c}\text { MIC } \\
\# 1 \\
\end{array}$ & $\begin{array}{c}\mathrm{MIC} \\
\# 2\end{array}$ & $\begin{array}{c}\mathrm{MIC} \\
\# 1 \\
\end{array}$ & $\begin{array}{c}\mathrm{MIC} \\
\# 2\end{array}$ & $\begin{array}{c}\text { MIC } \\
\# 1 \\
\end{array}$ & $\begin{array}{c}\mathrm{MIC} \\
\# 2\end{array}$ & $\begin{array}{c}\mathrm{MIC} \\
\# 1\end{array}$ & $\begin{array}{c}\mathrm{MIC} \\
\# 2\end{array}$ & $\begin{array}{c}\mathrm{MIC} \\
\# 1\end{array}$ & $\begin{array}{c}\text { MIC } \\
\# 2 \\
\end{array}$ & $\begin{array}{c}\mathrm{MIC} \\
\# 1\end{array}$ & $\begin{array}{c}\mathrm{MIC} \\
\# 2 \\
\end{array}$ & & \\
\hline 31 & 0.5 & 0.5 & 0.125 & 0.125 & $>64$ & 64 & 0.5 & 0.5 & 0.5 & 1 & 0.25 & 0.25 & G448E & $\uparrow C D R 1$ \\
\hline 32 & $\leq 0.015$ & 0.03 & $\leq 0.015$ & $\leq 0.015$ & 8 & 8 & 0.06 & 0.06 & 0.125 & 0.25 & 0.06 & 0.125 & G464S & $\begin{array}{c}\uparrow C D R 1, \\
M D R 1\end{array}$ \\
\hline 33 & 0.125 & 0.125 & $\leq 0.015$ & $\leq 0.015$ & 32 & 32 & 0.5 & 0.5 & 0.25 & 0.5 & 0.25 & 0.25 & F145L E266D & $\uparrow C D R 1$ \\
\hline 34 & 0.125 & 0.125 & 0.06 & 0.06 & 64 & 16 & 0.5 & 0.25 & 0.5 & 0.5 & 0.25 & 0.5 & G450E & $\uparrow C D R 1$ \\
\hline 35 & 0.015 & 0.03 & 0.03 & 0.03 & 16 & 16 & 0.06 & 0.125 & 0.25 & 0.25 & 0.125 & 0.25 & D446E & $\begin{array}{c}\uparrow E R G 11, \\
M D R 1\end{array}$ \\
\hline 36 & 0.5 & 0.25 & 0.06 & 0.06 & 64 & 64 & 0.5 & 1 & 0.25 & 0.25 & 0.125 & 0.25 & None & $\begin{array}{c}\uparrow E R G 11, \\
C D R 1\end{array}$ \\
\hline 37 & 0.125 & 0.125 & 0.06 & 0.06 & 32 & 64 & 0.5 & 0.5 & 0.25 & 0.25 & 0.125 & 0.25 & None & $\begin{array}{c}\uparrow E R G 11, \\
C D R 1\end{array}$ \\
\hline 38 & 1 & 0.5 & 0.125 & 0.06 & $>64$ & $>64$ & 2 & 1 & 0.25 & 0.5 & 0.25 & 0.5 & G464S & $\begin{array}{c}\uparrow E R G 11, \\
C D R 1\end{array}$ \\
\hline 39 & 1 & 1 & 0.125 & 0.125 & $>64$ & $>64$ & 2 & 2 & 1 & 1 & 0.5 & 1 & G464S & $\begin{array}{c}\uparrow E R G 11, \\
C D R 1\end{array}$ \\
\hline 40 & 0.5 & 0.25 & 0.125 & 0.125 & $>64$ & $>64$ & 1 & 1 & 0.5 & 0.5 & 0.25 & 0.5 & F449V & $\begin{array}{c}\uparrow E R G 11, \\
C D R 1\end{array}$ \\
\hline 41 & 0.06 & 0.06 & 0.06 & 0.03 & 64 & 64 & 0.25 & 0.25 & 0.25 & 0.25 & 0.125 & 0.25 & $\mathrm{~S} 405 \mathrm{~F}$ & $\begin{array}{c}\uparrow E R G 11, \\
M D R 1\end{array}$ \\
\hline 42 & 0.25 & 0.125 & 0.06 & 0.06 & 64 & 32 & 1 & 0.5 & 0.25 & 0.25 & 0.125 & 0.25 & G464S & $\uparrow C D R 1$ \\
\hline 43 & 0.03 & 0.06 & $\leq 0.015$ & 0.06 & 8 & 8 & 0.25 & 0.5 & 0.25 & 0.25 & 0.06 & 0.125 & D278N G464S & $\uparrow C D R 1$ \\
\hline 44 & 0.5 & 0.25 & 0.03 & 0.03 & $>64$ & 64 & 1 & 1 & 0.25 & 0.5 & 0.25 & 0.5 & $\mathrm{D} 446 \mathrm{E}$ & $\uparrow C D R 1$ \\
\hline 45 & 0.5 & 0.25 & 0.06 & 0.06 & 64 & 32 & 1 & 1 & 0.25 & 0.5 & 0.125 & 0.25 & E266D G464S & $\uparrow C D R 1$ \\
\hline
\end{tabular}


Table B-1. (Continued)

\begin{tabular}{|c|c|c|c|c|c|c|c|c|c|c|c|c|c|c|}
\hline \multirow{2}{*}{$\begin{array}{l}\text { Isolate } \\
\text { ID }\end{array}$} & \multicolumn{2}{|c|}{ VT-1161 } & \multicolumn{2}{|c|}{ VT-1598 } & \multicolumn{2}{|c|}{ Fluconazole } & \multicolumn{2}{|c|}{ Voriconazole } & \multicolumn{2}{|c|}{ Itraconazole } & \multicolumn{2}{|c|}{ Posaconazole } & \multirow{2}{*}{$\begin{array}{c}\text { ERG11 } \\
\text { mutations }^{\text {a }}\end{array}$} & \multirow{2}{*}{$\begin{array}{c}\text { Other } \\
\text { Resistance } \\
\text { Mechanisms }^{\text {b }}\end{array}$} \\
\hline & $\begin{array}{c}\mathrm{MIC} \\
\# 1\end{array}$ & $\underset{\# 2}{\mathrm{MIC}}$ & $\begin{array}{c}\mathrm{MIC} \\
\# 1\end{array}$ & $\underset{\# 2}{\mathrm{MIC}}$ & $\begin{array}{c}\mathrm{MIC} \\
\# 1\end{array}$ & $\begin{array}{c}\mathrm{MIC} \\
\# 2\end{array}$ & $\begin{array}{c}\mathrm{MIC} \\
\# 1\end{array}$ & $\begin{array}{c}\mathrm{MIC} \\
\# 2\end{array}$ & $\begin{array}{c}\mathrm{MIC} \\
\# 1\end{array}$ & $\begin{array}{c}\mathrm{MIC} \\
\# 2\end{array}$ & $\begin{array}{c}\mathrm{MIC} \\
\# 1\end{array}$ & $\begin{array}{c}\mathrm{MIC} \\
\# 2\end{array}$ & & \\
\hline 46 & $\leq 0.015$ & $\leq 0.015$ & $\leq 0.015$ & $\leq 0.015$ & 32 & 64 & $\leq 0.03$ & $\leq 0.03$ & $\leq 0.03$ & $\leq 0.03$ & $\leq 0.03$ & $\leq 0.03$ & $\begin{array}{c}\text { G307S, L403F } \\
\text { G448R }\end{array}$ & --- \\
\hline 47 & 0.125 & 0.125 & 0.03 & $\leq 0.015$ & $>64$ & 64 & 0.5 & 0.5 & 0.25 & 0.25 & 0.125 & 0.25 & G450E & $\begin{array}{c}\uparrow E R G 11, \\
C D R 1\end{array}$ \\
\hline 48 & 2 & 2 & 0.06 & 0.03 & 32 & 32 & 0.5 & 0.5 & 0.25 & 0.25 & 0.06 & 0.125 & Y132F & $\uparrow C D R 1$ \\
\hline 49 & 0.125 & 0.125 & 0.06 & 0.125 & $>64$ & $>64$ & 0.5 & 0.5 & 0.5 & 0.5 & 0.25 & 0.5 & V437I, Y447S & $\begin{array}{c}\uparrow E R G 11, \\
C D R 1\end{array}$ \\
\hline 50 & 1 & 0.5 & 0.06 & 0.06 & $>64$ & $>64$ & 2 & 1 & 0.25 & 0.5 & 0.06 & 0.25 & Y132F K143R & $\uparrow C D R 1$ \\
\hline 51 & 0.5 & 0.5 & 0.06 & 0.06 & $>64$ & 64 & 2 & 1 & 0.125 & 0.25 & 0.125 & 0.25 & D278N G464S & $\uparrow C D R 1$ \\
\hline 53 & $\leq 0.015$ & $\leq 0.015$ & $\leq 0.015$ & $\leq 0.015$ & 1 & 1 & $\leq 0.03$ & $\leq 0.03$ & 0.125 & 0.25 & 0.25 & 0.25 & None & $\uparrow E R G 11$ \\
\hline 54 & $\leq 0.015$ & $\leq 0.015$ & $\leq 0.015$ & $\leq 0.015$ & 32 & 32 & $\leq 0.03$ & 0.06 & 0.06 & 0.125 & $\leq 0.03$ & 0.06 & G307S G450E & $\begin{array}{c}\uparrow E R G 11, \\
M D R 1\end{array}$ \\
\hline 55 & 4 & 8 & $>8$ & $>8$ & $>64$ & $>64$ & $>16$ & $>16$ & $>16$ & $>16$ & $>16$ & $>16$ & None & --- \\
\hline 56 & 4 & 8 & $>8$ & $>8$ & $>64$ & 64 & $>16$ & $>16$ & $>16$ & $>16$ & $>16$ & $>16$ & None & $\uparrow C D R 1$ \\
\hline 58 & 4 & 4 & $>8$ & $>8$ & $>64$ & $>64$ & $>16$ & 0.5 & $>16$ & $>16$ & $>16$ & $>16$ & None & $\begin{array}{c}\uparrow E R G 11, \\
C D R 1\end{array}$ \\
\hline 59 & 0.5 & 0.5 & 0.125 & 0.06 & $>64$ & $>64$ & 2 & 2 & 0.25 & 0.25 & 0.06 & 0.125 & $\begin{array}{c}\text { Y132F, T229A, } \\
\text { F449I }\end{array}$ & $\begin{array}{c}\uparrow E R G 11, \\
C D R 1\end{array}$ \\
\hline 60 & 4 & $>8$ & $>8$ & $>8$ & $>64$ & $>64$ & $>16$ & $>16$ & $>16$ & $>16$ & $>16$ & $>16$ & None & $\begin{array}{c}E R G 3^{\mathrm{W} 131^{*}}, \\
\uparrow E R G 11\end{array}$ \\
\hline 61 & 0.5 & 0.5 & 0.03 & 0.06 & $>64$ & $>64$ & 1 & 1 & 0.25 & 0.5 & 0.125 & 0.25 & Y132F K143R & $\uparrow C D R 1$ \\
\hline 62 & 0.125 & 0.25 & $\leq 0.015$ & $\leq 0.015$ & 32 & 32 & 0.5 & 0.5 & 0.25 & 0.5 & 0.125 & 0.25 & D278N G464S & $\uparrow C D R 1$ \\
\hline
\end{tabular}


Table B-1. (Continued)

\begin{tabular}{|c|c|c|c|c|c|c|c|c|c|c|c|c|c|c|}
\hline \multirow{2}{*}{$\begin{array}{l}\text { Isolate } \\
\text { ID }\end{array}$} & \multicolumn{2}{|c|}{ VT-1161 } & \multicolumn{2}{|c|}{ VT-1598 } & \multicolumn{2}{|c|}{ Fluconazole } & \multicolumn{2}{|c|}{ Voriconazole } & \multicolumn{2}{|c|}{ Itraconazole } & \multicolumn{2}{|c|}{ Posaconazole } & \multirow{2}{*}{$\begin{array}{c}\text { ERG11 } \\
\text { mutations }^{\mathrm{a}}\end{array}$} & \multirow{2}{*}{$\begin{array}{c}\text { Other } \\
\text { Resistance } \\
\text { Mechanisms }\end{array}$} \\
\hline & $\begin{array}{c}\mathrm{MIC} \\
\# 1\end{array}$ & $\begin{array}{c}\mathrm{MIC} \\
\# 2\end{array}$ & $\begin{array}{c}\text { MIC } \\
\# 1\end{array}$ & $\begin{array}{c}\text { MIC } \\
\# 2 \\
\end{array}$ & $\begin{array}{c}\text { MIC } \\
\# 1\end{array}$ & $\begin{array}{c}\mathrm{MIC} \\
\# 2\end{array}$ & $\begin{array}{c}\mathrm{MIC} \\
\# 1\end{array}$ & $\begin{array}{c}\text { MIC } \\
\# 2\end{array}$ & $\begin{array}{c}\text { MIC } \\
\# 1\end{array}$ & $\begin{array}{c}\text { MIC } \\
\# 2\end{array}$ & $\begin{array}{c}\mathrm{MIC} \\
\# 1\end{array}$ & $\begin{array}{c}\mathrm{MIC} \\
\# 2\end{array}$ & & \\
\hline 63 & 0.06 & 0.125 & 0.125 & 0.06 & 32 & 32 & 0.25 & 0.25 & 0.5 & 0.5 & 0.25 & 0.25 & G450E & $\begin{array}{c}\uparrow C D R 1, \\
M D R 1\end{array}$ \\
\hline 64 & 0.25 & 0.125 & 0.125 & 0.06 & 32 & 32 & 0.5 & 0.25 & 0.5 & 0.5 & 0.25 & 0.25 & $\mathrm{G} 450 \mathrm{E}$ & $\begin{array}{l}\uparrow E R G 11, \\
M D R 1\end{array}$ \\
\hline 65 & 4 & 2 & $>8$ & 0.5 & 64 & 64 & 1 & 0.5 & 1 & 2 & 0.5 & 0.5 & G448E & $\begin{array}{c}\uparrow E R G 11, \\
C D R 1\end{array}$ \\
\hline 66 & 0.5 & 0.5 & 0.25 & 0.125 & 64 & 64 & 0.5 & 0.5 & 0.5 & 0.5 & 0.25 & 0.5 & G448E & $\uparrow C D R 1$ \\
\hline 67 & 0.5 & 0.5 & $>8$ & 0.25 & $>64$ & 64 & 1 & 1 & 1 & 1 & 0.5 & 0.5 & G448E & $\begin{array}{c}\uparrow E R G 11, \\
C D R 1\end{array}$ \\
\hline 68 & 0.5 & 0.5 & $\leq 0.015$ & $\leq 0.015$ & $>64$ & $>64$ & 0.5 & 0.5 & 0.25 & 0.5 & $\leq 0.03$ & 0.06 & $\begin{array}{l}\text { Y132F, E266D, } \\
\text { I471M, V488I }\end{array}$ & $\begin{array}{l}\uparrow E R G 11, \\
M D R 1\end{array}$ \\
\hline 69 & 0.5 & 1 & 0.25 & 0.25 & 64 & 64 & 1 & 1 & 1 & 1 & 0.5 & 0.5 & G448E & $\begin{array}{c}\uparrow E R G 11, \\
C D R 1\end{array}$ \\
\hline 70 & 0.06 & 0.03 & $\leq 0.015$ & $\leq 0.015$ & 32 & 32 & 0.5 & 0.5 & 0.25 & 0.5 & 0.125 & 0.125 & M258L, G464S & --- \\
\hline 71 & 0.25 & 0.125 & 0.06 & 0.06 & $>64$ & 64 & 0.5 & 0.5 & 0.5 & 0.5 & 0.125 & 0.125 & $\begin{array}{c}\text { Y132F, V437I, } \\
\text { F449L }\end{array}$ & $\begin{array}{c}\uparrow E R G 11, \\
C D R 1\end{array}$ \\
\hline 72 & 0.25 & 0.125 & 0.06 & 0.125 & 16 & 16 & 0.25 & 0.25 & 0.5 & 0.5 & 0.25 & 0.25 & $\begin{array}{c}\mathrm{A} 114 \mathrm{~V}, \\
\mathrm{D} 153 \mathrm{E}, \\
\mathrm{E} 266 \mathrm{D}, \mathrm{G} 450 \mathrm{E}\end{array}$ & $\uparrow C D R 1$ \\
\hline
\end{tabular}

a(h) indicates heterozygous mutation

${ }^{\mathrm{b}} \uparrow$ denotes increased expression of the gene in question

Notes: Low antifungal MICs (within a two-fold increase of that of the fluconazole-susceptible isolates) against fluconazole-resistant isolates are highlighted in yellow. 
Table B-2. MICs for VT-1161, VT-1598, fluconazole, voriconazole, itraconazole, and posaconazole against ERG11 mutant strains

\begin{tabular}{|c|c|c|c|c|c|c|c|c|c|c|c|c|}
\hline \multirow[b]{2}{*}{$\begin{array}{l}\text { Erg11 } \\
\text { substitution }\end{array}$} & \multicolumn{2}{|c|}{ VT-1598 } & \multicolumn{2}{|c|}{ VT-1161 } & \multicolumn{2}{|c|}{ Fluconazole } & \multicolumn{2}{|c|}{ Itraconazole } & \multicolumn{2}{|c|}{ Posaconazole } & \multicolumn{2}{|c|}{ Voriconazole } \\
\hline & $\begin{array}{c}\text { MIC } \\
\# 1 \\
\end{array}$ & $\begin{array}{c}\mathrm{MIC} \\
\# 2 \\
\end{array}$ & $\begin{array}{c}\text { MIC } \\
\# 1 \\
\end{array}$ & $\begin{array}{c}\mathrm{MIC} \\
\# 2 \\
\end{array}$ & $\begin{array}{c}\text { MIC } \\
\# 1 \\
\end{array}$ & $\begin{array}{c}\mathrm{MIC} \\
\# 2 \\
\end{array}$ & $\begin{array}{c}\text { MIC } \\
\# 1 \\
\end{array}$ & $\begin{array}{c}\text { MIC } \\
\# 2 \\
\end{array}$ & $\begin{array}{c}\text { MIC } \\
\# 1 \\
\end{array}$ & $\begin{array}{c}\mathrm{MIC} \\
\# 2 \\
\end{array}$ & $\begin{array}{c}\text { MIC } \\
\# 1 \\
\end{array}$ & $\begin{array}{c}\text { MIC } \\
\# 2 \\
\end{array}$ \\
\hline Wildtype $^{\mathrm{a}}$ & $\leq 0.015$ & $\leq 0.015$ & $\leq 0.015$ & $\leq 0.015$ & 0.5 & 0.5 & 0.125 & 0.125 & $\leq 0.03$ & $\leq 0.03$ & $\leq 0.03$ & $\leq 0.03$ \\
\hline Y132F & $\leq 0.015$ & $\leq 0.015$ & 0.25 & 0.125 & 4 & 8 & 0.125 & 0.125 & $\leq 0.03$ & $\leq 0.03$ & 0.25 & 0.125 \\
\hline $\mathrm{Y} 132 \mathrm{H}$ & $\leq 0.015$ & $\leq 0.015$ & $\leq 0.015$ & $\leq 0.015$ & 2 & 1 & 0.125 & 0.125 & $\leq 0.03$ & $\leq 0.03$ & 0.125 & 0.06 \\
\hline K143R & $\leq 0.015$ & $\leq 0.015$ & $\leq 0.015$ & $\leq 0.015$ & 2 & 4 & 0.06 & 0.125 & $\leq 0.03$ & $\leq 0.03$ & $\leq 0.03$ & $\leq 0.03$ \\
\hline F145L & 0.03 & $\leq 0.015$ & $\leq 0.015$ & $\leq 0.015$ & 4 & 4 & 0.25 & 0.25 & $\leq 0.03$ & $\leq 0.03$ & 0.125 & 0.125 \\
\hline E266D & $\leq 0.015$ & $\leq 0.015$ & $\leq 0.015$ & $\leq 0.015$ & 1 & 1 & 0.125 & 0.125 & $\leq 0.03$ & $\leq 0.03$ & 0.06 & 0.06 \\
\hline $\mathrm{D} 278 \mathrm{~N}$ & $\leq 0.015$ & $\leq 0.015$ & $\leq 0.015$ & $\leq 0.015$ & 2 & 1 & 0.125 & 0.125 & $\leq 0.03$ & $\leq 0.03$ & 0.125 & 0.06 \\
\hline $\mathrm{S} 405 \mathrm{~F}$ & 0.03 & $\leq 0.015$ & $\leq 0.015$ & $\leq 0.015$ & 4 & 4 & 0.25 & 0.25 & $\leq 0.03$ & $\leq 0.03$ & 0.125 & 0.125 \\
\hline G448E & $\leq 0.015$ & $\leq 0.015$ & $\leq 0.015$ & $\leq 0.015$ & 1 & 2 & 0.125 & 0.25 & $\leq 0.03$ & $\leq 0.03$ & $\leq 0.03$ & 0.06 \\
\hline F449V & $\leq 0.015$ & $\leq 0.015$ & $\leq 0.015$ & $\leq 0.015$ & 2 & 4 & 0.125 & 0.125 & $\leq 0.03$ & $\leq 0.03$ & 0.06 & $\leq 0.03$ \\
\hline G450E & $\leq 0.015$ & $\leq 0.015$ & $\leq 0.015$ & $\leq 0.015$ & 2 & 2 & 0.125 & 0.25 & $\leq 0.03$ & $\leq 0.03$ & 0.06 & 0.06 \\
\hline G464S & $\leq 0.015$ & $\leq 0.015$ & $\leq 0.015$ & $\leq 0.015$ & 2 & 2 & 0.125 & 0.125 & $\leq 0.03$ & $\leq 0.03$ & 0.06 & 0.06 \\
\hline D466E & $\leq 0.015$ & $\leq 0.015$ & $\leq 0.015$ & $\leq 0.015$ & 1 & 1 & 0.125 & 0.25 & $\leq 0.03$ & $\leq 0.03$ & 0.125 & 0.06 \\
\hline Y132F, K143R & $\leq 0.015$ & $\leq 0.015$ & 0.06 & 0.125 & 32 & 64 & 0.125 & 0.125 & $\leq 0.03$ & $\leq 0.03$ & 0.25 & 0.25 \\
\hline Y132F, F145L & 0.03 & 0.03 & $\leq 0.015$ & 0.06 & 16 & 32 & 0.25 & 0.25 & $\leq 0.03$ & $\leq 0.03$ & 0.5 & 1 \\
\hline D278N, G464S & 0.03 & $\leq 0.015$ & $\leq 0.015$ & $\leq 0.015$ & 4 & 8 & 0.25 & 0.25 & $\leq 0.03$ & $\leq 0.03$ & 0.25 & 0.25 \\
\hline G450E, I483V & $\leq 0.015$ & $\leq 0.015$ & $\leq 0.015$ & $\leq 0.015$ & 2 & 2 & 0.125 & 0.125 & $\leq 0.03$ & $\leq 0.03$ & 0.125 & 0.125 \\
\hline
\end{tabular}

${ }^{a}$ From azole-susceptible parent strain SC5314 
Table B-3. Primers used in Chapter 5

\begin{tabular}{|c|c|}
\hline Primer name by purpose & Sequence $^{\mathrm{a}}$ \\
\hline \multicolumn{2}{|l|}{ Amplification } \\
\hline CaERG11_AF_(ApaI) & $5^{\prime}$ - GGGCCCGGGTTATTTGAGAACAGCC - 3' \\
\hline CaERG11_BR_(XhoI) & 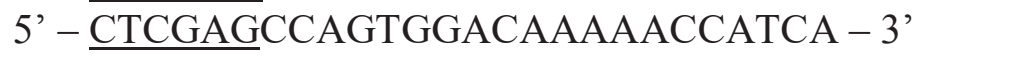 \\
\hline CaERG11_C-F’ & 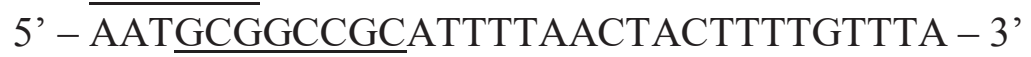 \\
\hline CaERG11_D-R' & 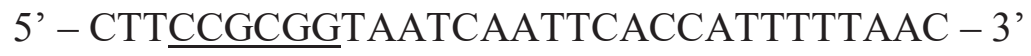 \\
\hline \multicolumn{2}{|l|}{ Sequencing } \\
\hline CaERG11SeqA & 5' - GCCACCACACCCTATGGCTATT - 3' \\
\hline CaERG11SeqB & $5^{\prime}$ - TATTTTCACTGCTTCAAGATCT - ${ }^{\prime}$ \\
\hline CaERG11SeqC & $5^{\prime}$ - CCAAAAGGTCATTATGTTTTAG - 3' \\
\hline CaERG11SeqD & 5' - CATACAAGTTTCTCTTTTTTCC - 3’ \\
\hline CaERG11SeqE & $5^{\prime}$ - CATTTAGGTGAAAAACCTCATT - 3' \\
\hline CaERG11SeqF & 5' - TACTCCAGTTTTCGGTAAAGGG - 3' \\
\hline \multicolumn{2}{|l|}{ Short, overlapping extension } \\
\hline CaERG11_1F & 5' - CAATCTATACGACGACATTTAACTTTTT - 3' \\
\hline $\begin{array}{l}\text { CaERG11S SOE- } \\
\text { 3R Y132H }\end{array}$ & 5' - GACAATCATGAATAACCCCTTTACCGAAAA - 3' \\
\hline $\begin{array}{l}\text { CaERG11SOE- } \\
2 F_{-}{ }_{132 H}\end{array}$ & 5' - GGGGTTATTCATGATTGTCCAAATTCCAGA - 3' \\
\hline CaĒER11_4R & 5' - GCTGTTGTTGTTGTTGAAAGAAA - 3' \\
\hline CaERG11S̄OE-5F & 5' - ATTATTGAAAGAAAAAGGTGGTGATTTG - 3' \\
\hline CaERG11SOE-6R & 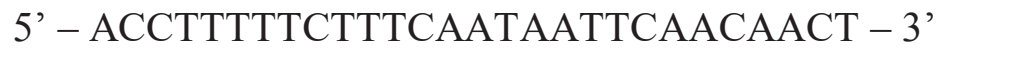 \\
\hline \multicolumn{2}{|l|}{ Real-time qPCR } \\
\hline CaCDR1-F_qPCR & 5' - ATTCTAAGATGTCGTCGCAAGATG - 3' \\
\hline CaCDR1-R_qPCR & 5' - AGTTCTGGCTAAATTCTGAATGTTTTC - 3' \\
\hline CaERG11-F_qPCR & $5^{\prime}$ - CСССТАTTAATTTTGTTTTCССТАATTTAC - 3' \\
\hline CaERG11-R_qPCR & 5' - CACGTTCTCTTCTCAGTTTAATTTCTTTC - 3' \\
\hline CaBMR1-F_qPCR & 5' - ACATAAATACTTTGCCCATCCAGAA - 3' \\
\hline CaBMR1-R_qPCR & 5' - AAGAGTTGGTTTGTAATCGGCTAAA - 3' \\
\hline CaACT1-FW̄D_qPCR & 5' - ACGGTGAAGAAGTTGCTGCTTTAGTT - 3' \\
\hline CaACT1-rvs_qPCR & 5' - CGTCGTCACCGGCAAAA - 3' \\
\hline
\end{tabular}

${ }^{a}$ Underlined nucleotide indicates introduction of a restriction site sequence 


\section{VITA}

Andrew (Andy) Tadashi Nishimoto was born in Long Beach, California in 1984 to S. Ken and Miyako Nishimoto. He enthusiastically attended Farmington Elementary, Houston Middle/High School in Germantown, Tennessee and graduated as valedictorian in 2003. He graduated with a Bachelor of the Arts in Molecular Biology from Pomona College in Claremont, California in 2007 and enrolled in the PharmD/PhD dual degree program at the University of Tennessee Health Science Center in 2009. He earned his Doctor of Pharmacy degree with Honors in 2013 and completed a one year pharmacy practice residency at Methodist University Hospital in Memphis, TN, earning his BCPS certification in 2015. Andy anticipates graduation from the Pharmaceutical Sciences program in the University of Tennessee College of Graduate Health Sciences with a Doctor of Philosophy degree in 2019. 